\title{
Amino Acid-derived Chiral Aminobenzimidazole Manganese Catalysts for Asymmetric Transfer Hydrogenation of Ketones
}

Lixian Wang, ${ }^{\dagger, \dagger}$ Jin Lin, ${ }^{\dagger}$ Qiangsheng Sun,${ }^{\dagger}$ Chungu Xia, ${ }^{\dagger}$ and Wei Sun ${ }^{*}, \dagger$

†State Key Laboratory for Oxo Synthesis and Selective Oxidation, Center for Excellence in Molecular Synthesis, Suzhou Research Institute of LICP, Lanzhou Institute of Chemical Physics (LICP), Chinese Academy of Sciences, Lanzhou 730000, China

\#University of Chinese Academy of Sciences, Beijing 100049, China

*To whom correspondence should be addressed.

E-mail:wsun@licp.cas.cn 


\section{Table of Contents}

1. General information

2. Synthesis and characterization of chiral aminobenzimidazole ligands and the corresponding Mn complexes

\section{S4-S12}

3. Reaction conditions optimization for ATH of acetophenone

S12-S18

4. Synthesis and characterization of Mn-H from complex Mn-1

5. KIE experiment for the ATH of acetophenone with Mn-1 as catalyst

6. Copies of NMR spectra

7. Copies of HPLC and GC spectra

S82-S112

8. Computational details

S113

9. References

S116 


\section{General information.}

All solvents and commercially available compounds were used as received unless stated otherwise. Analytical thin-layer chromatography (TLC) was carried out using $0.25 \mathrm{~mm}$ commercial silica gel plates. Visualization was accomplished with UV light. Purification of reactions was carried out by flash chromatography using Huanghai silica gel (300-400 mesh).

NMR spectra were recorded at $298 \mathrm{~K}$ on a Bruker avance 400 . Chemical shift values for ${ }^{1} \mathrm{H}$ and ${ }^{13} \mathrm{C}$ are reported as $\delta$ values (ppm) relative to the deuterated solvent and coupling constants $(J)$ in Hz. High resolution mass spectroscopy experiments were carried out with Bruker Daltonics microTOF-Q ${ }^{\mathrm{II}}$ mass spectrometer. X-ray crystallographic data were collected on a Bruker SMART CCD1000 diffractometer with graphitemonochromated Mo K $\alpha$ radiation $(\lambda=0.71073 \AA$ ) at 296(2)K. GC-MS data were recorded in an Agilent Technologies 7890 A GC/5975 MS (EI) containing a HP-5MS column $(0.25 \mathrm{~mm} \times 30 \mathrm{~m}$, Film: $0.25 \mu \mathrm{m})$. High pressure liquid chromatography (HPLC) analysis was performed on a Waters-Breeze instrument (2487 Dual $\lambda$ Absorbance Detector and 1525 Binary HPLC Pump) or a SHMADZU system (SHMADZU LC-20AT pump, SHIMADZU LC-20A Absorbance Detector). Chiralpak OD-H, AD-H columns were purchased from Daicel Chemical Industries, LTD. Gas chromatography (GC) analysis was performed on a Agilent 7890 GC with a CP-Chirasil-Dex CB column. 


\section{Synthesis and characterization of chiral bidentate benzimidazole ligands and the corresponding Mn complexes}

\subsection{Synthesis of ligands L1-L10}

Method A:
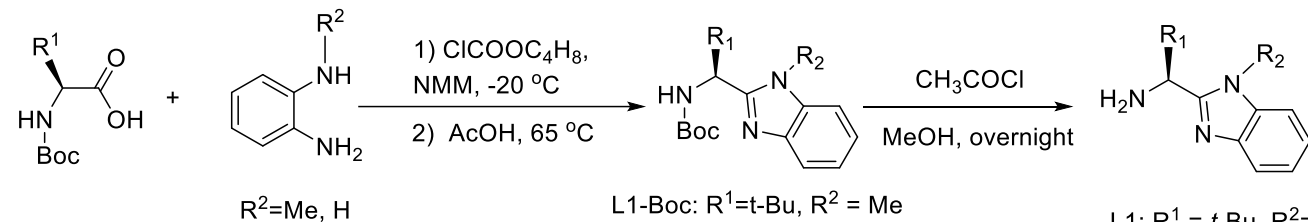

L1-Boc: $R^{1}=t-B u, R^{2}=M e$ $\mathrm{L}^{\mathrm{NH}}{ }^{\mathrm{B}}$ Boc: $\mathrm{R}^{1}=t-\mathrm{Bu}, \mathrm{R}^{2}=\mathrm{H}$

$\mathrm{L} 1: \mathrm{R}^{1}=t-\mathrm{Bu}, \mathrm{R}^{2}=\mathrm{Me}$

L3-Boc: $R^{1}=i-P r, R^{2}=M e$

L3: $\mathrm{R}^{1}=i-\mathrm{Pr}, \mathrm{R}^{2}=\mathrm{Me}$

L4-Boc: $\mathrm{R}^{1}=1$-adamantyl, $\mathrm{R}^{2}=\mathrm{Me}$

L4: $R^{1}=1$-adamantyl, $R^{2}=M e$

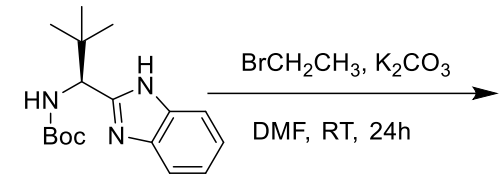

$$
\mathrm{L}^{\mathrm{NH}}-\mathrm{BoC}
$$<smiles>CCn1c(C(NC(=O)O)C(C)(C)C)nc2ccccc21</smiles>

L2-Boc

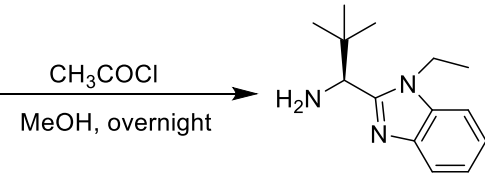

L2

Compounds L1-Boc, L1 ${ }^{\mathrm{NH}}$-Boc, L3-Boc, L4-Boc and L1-L5 were prepared according to the reported method from the corresponding Bocprotected amino acids. ${ }^{1}$

(S)-2,2-dimethyl-1-(1-methyl-1H-benzo[d]imidazol-2-yl)propan-1-amine (L1)<smiles>Cn1c([C@@H](N)C(C)(C)C)nc2ccccc21</smiles>

White solid, 93\% isolated yield, $[\mathrm{a}]_{\boldsymbol{D}}^{\mathbf{2 0}}=+54.8\left(\mathrm{c} 0.800 \mathrm{in} \mathrm{CHCl}_{3}\right),{ }^{1} \mathrm{H}$ NMR $\left(400 \mathrm{MHz}, \mathrm{CDCl}_{3}\right) \delta$ 7.77-7.76 (m, $\left.1 \mathrm{H}\right), 7.32-7.27(\mathrm{~m}, 3 \mathrm{H}), 3.91(\mathrm{~s}$, $1 \mathrm{H}), 3.78(\mathrm{~s}, 3 \mathrm{H}), 1.96(\mathrm{~s}, 2 \mathrm{H}), 1.05(\mathrm{~s}, 9 \mathrm{H}) .{ }^{13} \mathrm{C} \mathrm{NMR}\left(100 \mathrm{MHz}, \mathrm{CDCl}_{3}\right) \delta 156.95,142.27,135.30,122.17,122.02,119.36,109.29,56.66,36.76$, $30.52,26.41$. HRMS (ESI-MS) $[\mathrm{M}+\mathrm{H}]^{+}:$calculated for $\left[\mathrm{C}_{12} \mathrm{H}_{18} \mathrm{~N}_{3}\right]^{+}: 218.1652$, found: 218.1654 .

(S)-1-(1-ethyl-1H-benzo[d]imidazol-2-yl)-2,2-dimethylpropan-1-amine (L2)<smiles>CCn1c([C@H](N)C(C)(C)C)nc2ccccc21</smiles>

White solid, $89 \%$ isolated yield, $[\mathrm{a}]_{\boldsymbol{D}}^{\mathbf{2 0}}=+63.3\left(\mathrm{c} 1.23 \mathrm{in} \mathrm{CHCl}_{3}\right),{ }^{1} \mathrm{H} \mathrm{NMR}\left(400 \mathrm{MHz}, \mathrm{CDCl}_{3}\right) \delta$ 7.78-7.74 (m, 1H), 7.37-7.33 (m, 1H), 7.27-7.23 $(\mathrm{m}, 2 \mathrm{H}), 4.27(\mathrm{q}, J=7.2 \mathrm{~Hz}, 2 \mathrm{H}), 3.86(\mathrm{~s}, 1 \mathrm{H}), 1.84(\mathrm{~s}, 2 \mathrm{H}), 1.44(\mathrm{t}, J=8.0 \mathrm{~Hz}, 3 \mathrm{H}), 1.07(\mathrm{~s}, 9 \mathrm{H}) .{ }^{13} \mathrm{C} \mathrm{NMR}\left(100 \mathrm{MHz}, \mathrm{CDCl}_{3}\right) \delta 156.54,142.67$, 134.04, 122.06, 121.89, 119.55, 109.53, 56.62, 38.65, 36.42, 26.54, 15.21. HRMS (ESI-MS) $[\mathrm{M}+\mathrm{H}]^{+}:$calculated for $\left[\mathrm{C}_{14} \mathrm{H}_{22} \mathrm{~N}_{3}\right]^{+}: 232.1808$, found: 232.1811.

(S)-2-methyl-1-(1-methyl-1H-benzo[d]imidazol-2-yl)propan-1-amine (L3)<smiles>CC(C)[C@H](N)c1nc2ccccc2n1C</smiles> 
White solid, $87 \%$ isolated yield, $[\mathrm{a}]_{D}^{20}=+23.9\left(\mathrm{c} 1.00\right.$ in $\left.\mathrm{CHCl}_{3}\right),{ }_{1}^{1} \mathrm{H}$ NMR $\left(400 \mathrm{MHz}, \mathrm{CDCl}_{3}\right) \delta$ 7.80-7.76 (m, 1H), 7.39-7.34 (m, 1H), 7.33-7.27 $(\mathrm{m}, 2 \mathrm{H}), 3.95(\mathrm{~d}, J=8.0 \mathrm{~Hz}, 1 \mathrm{H}), 3.83(\mathrm{~s}, 3 \mathrm{H}), 2.27-2.15(\mathrm{~m}, 1 \mathrm{H}), 1.83(\mathrm{~s}, 2 \mathrm{H}), 1.10(\mathrm{~d}, J=6.4 \mathrm{~Hz}, 3 \mathrm{H}), 0.99(\mathrm{~d}, J=6.4 \mathrm{~Hz}, 3 \mathrm{H}) .{ }^{13} \mathrm{C} \mathrm{NMR}(100$ $\left.\mathrm{MHz}, \mathrm{CDCl}_{3}\right) \delta 157.69,142.27,135.80,122.23,121.96,119.38,109.13,54.83,34.32,29.98,19.99,18.09$. HRMS (ESI-MS) [M+H] ${ }^{+}$: calculated for $\left[\mathrm{C}_{12} \mathrm{H}_{18} \mathrm{~N}_{3}\right]^{+}: 204.1495$, found: 204.1495 .

\section{(1S)-((1r,3S)-adamantan-1-yl)(1-methyl-1H-benzo[d]imidazol-2-yl)methanamin (L4)}<smiles>Cn1c(C(N)C23CC4CC(CC(C4)C2)C3)nc2ccccc21</smiles>

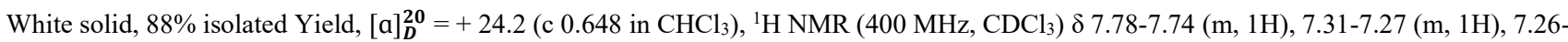
$7.22(\mathrm{~m}, 2 \mathrm{H}), 3.73(\mathrm{~s}, 3 \mathrm{H}), 3.71(\mathrm{~s}, 1 \mathrm{H}), 1.97(\mathrm{~s}, 3 \mathrm{H}), 1.88-1.85(\mathrm{~m}, 5 \mathrm{H}), 1.68-1.57(\mathrm{~m}, 6 \mathrm{H}), 1.49-1.46(\mathrm{~m}, 3 \mathrm{H}) .{ }^{13} \mathrm{C} \mathrm{NMR}\left(100 \mathrm{MHz}, \mathrm{CDCl}_{3}\right) \delta$ $156.43,142.35,135.34,122.05,121.92,119.32,109.27,57.47,38.60,38.39,36.94,30.53,28.38$. HRMS (ESI-MS) [M+H] $]^{+}$: calculated for $\left[\mathrm{C}_{19} \mathrm{H}_{26} \mathrm{~N}_{3}\right]^{+}: 296.2121$, found: 296.2119 .

\section{(S)-1-methyl-2-(pyrrolidin-2-yl)-1H-benzo[d]imidazole (L5)}<smiles>Cn1c([C@@H]2CCCN2)nc2ccccc21</smiles>

Yellow oil, 83\% isolated Yield, [a] $]_{\boldsymbol{D}}^{\mathbf{2 0}}=-57.0\left(\mathrm{c} 0.200 \mathrm{in} \mathrm{CHCl}_{3}\right),{ }_{1}^{1} \mathrm{H}$ NMR $\left(400 \mathrm{MHz}, \mathrm{CDCl}_{3}\right) \delta$ 7.78-7.76 (m, 1H), 7.36-7.33 (m, 1H), 7.32-7.26 $(\mathrm{m}, 2 \mathrm{H}), 4.46(\mathrm{t}, J=7.2 \mathrm{~Hz}, 1 \mathrm{H}), 3.83(\mathrm{~s}, 3 \mathrm{H}), 3.36-3.30(\mathrm{~m}, 1 \mathrm{H}), 3.06-3.00(\mathrm{~m}, 1 \mathrm{H}), 2.51(\mathrm{~s}, 1 \mathrm{H}), 2.34-2.26(\mathrm{~m}, 1 \mathrm{H}), 2.17-2.08(\mathrm{~m}, 1 \mathrm{H}), 2.03(\mathrm{~m}$, $2 \mathrm{H}) .{ }^{13} \mathrm{C}$ NMR $\left(100 \mathrm{MHz}, \mathrm{CDCl}_{3}\right) \delta 156.54,142.06,136.35,122.34,121.92,119.40,108.98,55.44,47.59,31.74,29.84,26.39$. HRMS (ESI-MS) $[\mathrm{M}+\mathrm{H}]^{+}:$calculated for $\left[\mathrm{C}_{12} \mathrm{H}_{16} \mathrm{~N}_{3}\right]^{+}: 202.1339$, found: 202.1344 .
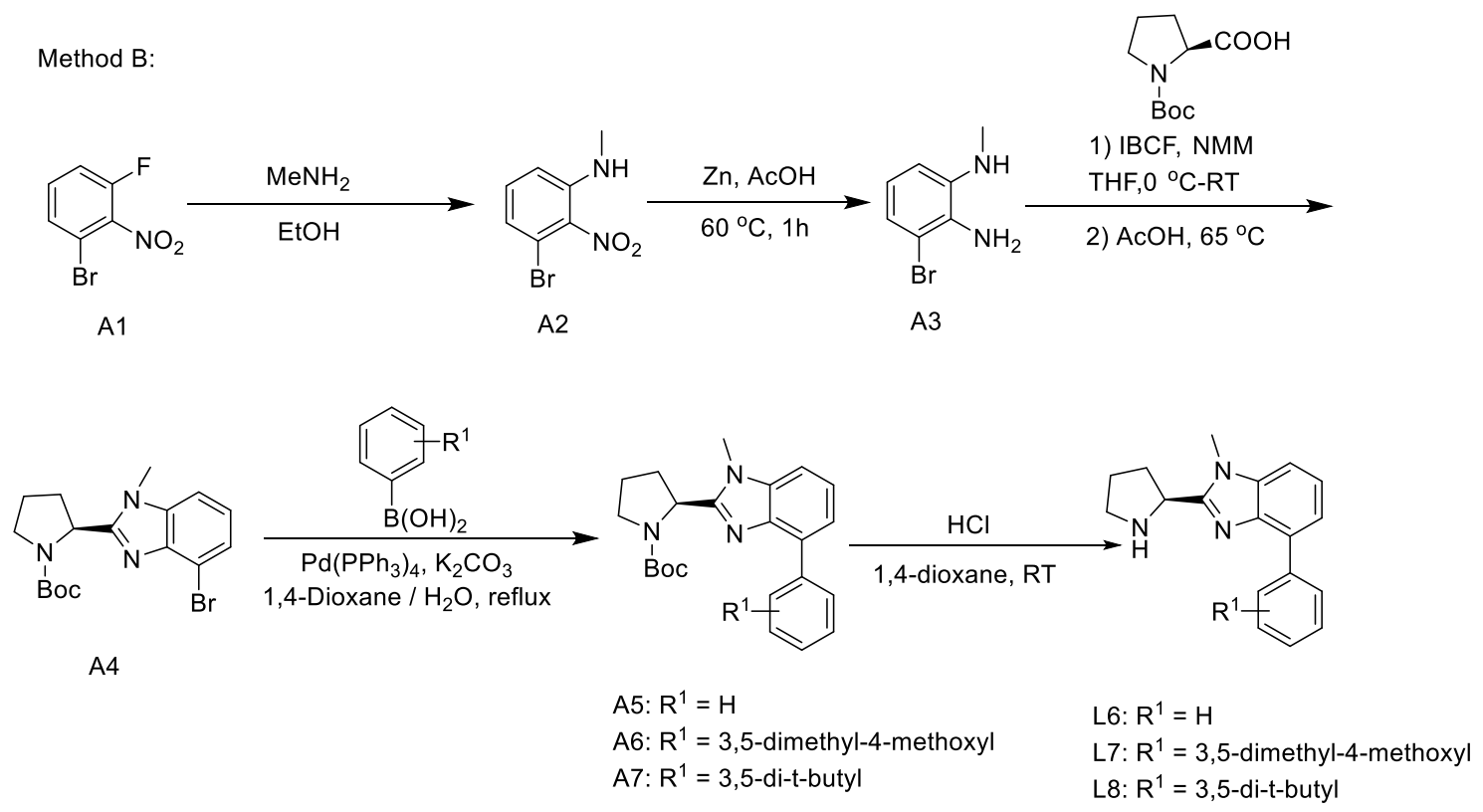

Compound $\mathrm{A} 3{ }^{2}$ and $\mathrm{A} 4{ }^{\mathrm{lb}}$ were synthesized from previously reported methods.

\section{Typical procedure for the synthesis of L6-L9.}

Under Ar atmosphere, benzeneboronic acid $(238 \mathrm{mg}, 1.95 \mathrm{mmol})$, compound $\mathrm{A} 4$ (570 mg, $1.5 \mathrm{mmol}), \mathrm{K}_{2} \mathrm{CO}_{3}(415 \mathrm{mg}, 3.0 \mathrm{mmol})$, and $\mathrm{Pd}\left(\mathrm{PPh}_{3}\right)_{4}(130 \mathrm{mg}, 0.11 \mathrm{mmol})$ were added into a $100 \mathrm{~mL}$ Schlenk tube containing 1,4-Dioxane $(12 \mathrm{~mL})$ and $\mathrm{H}_{2} \mathrm{O}(3 \mathrm{~mL})$. The reaction mixture was heated to reflux at $100{ }^{\circ} \mathrm{C}$ for $12 \mathrm{~h}$. After cooling to room temperature, the solvent was evaporated under reduced pressure and the residue was diluted with $\mathrm{H}_{2} \mathrm{O}$, extracted with $\mathrm{CH}_{2} \mathrm{Cl}_{2}$. The combined organic layer was washed with brine, dried over $\mathrm{Na}_{2} \mathrm{SO}_{4}$, and then evaporated to dryness. 
The crude product was purified by flash column chromatography on silica gel using PE/EA=1/4 as the eluent to give the product as a light yellow solid.

The derived benzimidazole (189 mg, $0.5 \mathrm{mmol})$ was dissolved in $\mathrm{CH}_{2} \mathrm{Cl}_{2}(2 \mathrm{~mL})$ and cooled to $0{ }^{\circ} \mathrm{C}$, after $1 \mathrm{ml}$ a solution of hydrogen chloride in 1,4-dioxane was added slowly. After $2 \mathrm{~h}$, the solvent was evaporated, and the residue was dissolved in $\mathrm{CH}_{2} \mathrm{Cl}_{2}$. $\mathrm{Next}_{5} \% \mathrm{NaHCO}_{3}$ was added and the $\mathrm{pH}$ was adjusted to around 9 , extracted with $\mathrm{CH}_{2} \mathrm{Cl}_{2}$. Then the solvent was evaporated, the residue was purified using column chromatography $(\mathrm{MeOH} / \mathrm{EA}=1: 5)$ to give the as a yellow solid ( $89 \%$ yield).

tert-butyl (S)-2-(4-bromo-1-methyl-1H-benzo[d]imidazol-2-yl)pyrrolidine-1-carboxylate(A4)<smiles>Cn1c([C@H]2CCCN2C(=O)OCc2ccccc2)nc2c(Br)cccc21</smiles>

Yellow gum, 33\% isolated yield, [a] $]_{\boldsymbol{D}}^{\mathbf{2 0}}=-60.7\left(\mathrm{c} 0.850\right.$ in $\left.\mathrm{CHCl}_{3}\right) .{ }^{1} \mathrm{H}$ NMR $\left(400 \mathrm{MHz}, \mathrm{CDCl}_{3}\right) \delta 7.43-7.38(\mathrm{~m}, 1 \mathrm{H}), 7.26(\mathrm{t}, J=8.0 \mathrm{~Hz}, 1 \mathrm{H})$, 7.17-7.06 (m, 1H), 5.16- $5.13(\mathrm{~m}, 1 \mathrm{H}), 3.88-3.75(\mathrm{~m}, 3 \mathrm{H}), 3.68-3.56(\mathrm{~m}, 2 \mathrm{H}), 2.50-2.43(\mathrm{~m}, 1 \mathrm{H}), 2.35-2.08(\mathrm{~m}, 2 \mathrm{H}), 2.02-1.96(\mathrm{~m}, 1 \mathrm{H}), 1.42(\mathrm{~s}$, 4H), 1.09 (s, 5H). ${ }^{13} \mathrm{C} \mathrm{NMR}\left(100 \mathrm{MHz}, \mathrm{CDCl}_{3}\right) \delta 156.04,155.85,154.70,154.08,141.28,141.12,136.36,125.08,124.91,123.10,122.89,113.09$, $113.02,108.64,108.10,79.93,79.73,55.82,53.28,46.90,33.19,32.20,30.51,28.46,28.02,24.71,24.11$. HRMS (ESI-MS) [M+H] $]^{+}:$calculated for $\left[\mathrm{C}_{17} \mathrm{H}_{23} \mathrm{BrN}_{3} \mathrm{O}_{2}\right]^{+}: 380.0968$, found: 380.0967 .

\section{(S)-1-methyl-4-phenyl-2-(pyrrolidin-2-yl)-1H-benzo[d]imidazole (L6)}<smiles>Cn1c([C@@H]2CCCN2)nc2c(-c3ccccc3)cccc21</smiles>

Yellow oil, 83\% isolated yield, [a] $]_{\boldsymbol{D}}^{\mathbf{2 0}}=-7.07\left(\mathrm{c} 0.920\right.$ in $\left.\mathrm{CHCl}_{3}\right) .{ }^{1} \mathrm{H}$ NMR $\left(400 \mathrm{MHz}, \mathrm{CDCl}_{3}\right) \delta$ 8.14-8.12 (m, 2H), 7.53-7.45 (m, 3H), 7.40-7.27 $(\mathrm{m}, 3 \mathrm{H}), 4.44(\mathrm{t}, J=8.0 \mathrm{~Hz}, 1 \mathrm{H}), 3.79(\mathrm{~s}, 3 \mathrm{H}), 3.33-3.27(\mathrm{~m}, 1 \mathrm{H}), 3.08(\mathrm{~s}, 1 \mathrm{H}), 3.02-2.96(\mathrm{~m}, 1 \mathrm{H}), 2.30-2.21(\mathrm{~m}, 1 \mathrm{H}), 2.08-1.87(\mathrm{~m}, 3 \mathrm{H}) .{ }^{13} \mathrm{C}$ NMR $\left(100 \mathrm{MHz}, \mathrm{CDCl}_{3}\right) \delta 156.26,139.85,138.55,137.32,131.94,129.36,128.37,127.27,122.52,121.23,108.11,55.80,47.48,31.81,29.98$, 26.35. HRMS (ESI-MS) $[\mathrm{M}+\mathrm{H}]^{+}$: calculated for $\left[\mathrm{C}_{19} \mathrm{H}_{24} \mathrm{~N}_{3}\right]^{+}: 278.1652$, found:278.1652.

\section{(S)-4-(4-methoxy-3,5-dimethylphenyl)-1-methyl-2-(pyrrolidin-2-yl)-1H-benzo[d]imidazole (L7)}<smiles>COc1c(C)cc(-c2cccc3c2nc(C2CCCN2)n3C)cc1C</smiles>

Yellow oil, 85\% isolated yield, [a] $]_{\boldsymbol{D}}^{20}=-3.69\left(\mathrm{c} 2.602\right.$ in $\left.\mathrm{CHCl}_{3}\right) .{ }^{1} \mathrm{H} \mathrm{NMR}\left(400 \mathrm{MHz}, \mathrm{CDCl}_{3}\right) \delta 7.72(\mathrm{~s}, 2 \mathrm{H}), 7.36-7.34(\mathrm{~m}, 1 \mathrm{H}), 7.27(\mathrm{t}, J=8.0$ $\mathrm{Hz}, 1 \mathrm{H}), 7.22-7.18(\mathrm{~m}, 1 \mathrm{H}), 4.39(\mathrm{t}, J=8.0 \mathrm{~Hz}, 1 \mathrm{H}), 3.75(\mathrm{~s}, 3 \mathrm{H}), 3.72(\mathrm{~s}, 3 \mathrm{H}), 3.29-3.23(\mathrm{~m}, 1 \mathrm{H}), 3.04(\mathrm{~s}, 1 \mathrm{H}), 2.98-2.92(\mathrm{~m}, 1 \mathrm{H}), 2.37(\mathrm{~s}, 6 \mathrm{H})$, 2.26-2.18 (m, 1H), $2.04-1.81(\mathrm{~m}, 3 \mathrm{H}) .{ }^{13} \mathrm{C}$ NMR $\left(100 \mathrm{MHz}, \mathrm{CDCl}_{3}\right) \delta 156.54,156.03,139.74,137.24,134.05,131.81,130.56,129.86,122.47$, $121.11,107.71,59.71,55.82,47.42,31.72,29.95,26.29,16.44$. HRMS (ESI-MS) $[\mathrm{M}+\mathrm{H}]^{+}$: calculated for $\left[\mathrm{C}_{21} \mathrm{H}_{26} \mathrm{~N}_{3} \mathrm{O}\right]^{+}: 336.2070$, found: 336.2061 .

(S)-4-(3,5-di-tert-butylphenyl)-1-methyl-2-(pyrrolidin-2-yl)-1H-benzo[d]imidazole (L8) 
<smiles>CC(C)Cc1cc(-c2cccc3c2nc(C2CCCN2)n3C)cc(C(C)(C)C)c1</smiles>

White solid, 84\% isolated yield, [a] $]_{D}^{20}=-5.69\left(\mathrm{c} 0.492\right.$ in $\left.\mathrm{CHCl}_{3}\right) .{ }^{1} \mathrm{H} \mathrm{NMR}\left(400 \mathrm{MHz}, \mathrm{CDCl}_{3}\right) \delta 7.98(\mathrm{~d}, \mathrm{~J}=2.0 \mathrm{~Hz}, 2 \mathrm{H}), 7.49-7.42(\mathrm{~m}, 2 \mathrm{H})$, $7.31(\mathrm{t}, \mathrm{J}=7.2 \mathrm{~Hz}, 1 \mathrm{H}), 7.21(\mathrm{dd}, \mathrm{J}=6.3,5.7 \mathrm{~Hz}, 1 \mathrm{H}), 4.43(\mathrm{dd}, \mathrm{J}=7.8,6.3 \mathrm{~Hz}, 1 \mathrm{H}), 3.74(\mathrm{~s}, 3 \mathrm{H}), 3.30-3.24(\mathrm{~m}, 1 \mathrm{H}), 3.00-2.93(\mathrm{~m}, 1 \mathrm{H}), 2.90$ (s, 1H), $2.27-2.18(\mathrm{~m}, 1 \mathrm{H}), 2.07(\mathrm{dt}, \mathrm{J}=12.7,6.6 \mathrm{~Hz}, 1 \mathrm{H}), 1.97-1.82(\mathrm{~m}, 2 \mathrm{H}), 1.41(\mathrm{~s}, 18 \mathrm{H}) .{ }^{13} \mathrm{C} \mathrm{NMR}\left(100 \mathrm{MHz}, \mathrm{CDCl}_{3}\right) \delta 156.33,150.35$, 139.84, 137.38, 137.32, 132.99, 124.03, 122.54, 121.43, 121.14, 107.78, 55.42, 47.63, 35.07, 31.67, 29.90, 26.37. HRMS (ESI-MS) [M+H] ${ }^{+}$: calculated for $\left[\mathrm{C}_{26} \mathrm{H}_{36} \mathrm{~N}_{3}\right]^{+}: 390.2904$, found: 390.2891 .

Method C:<smiles>CNc1cccc(Br)c1N</smiles>

A3
B1
B2

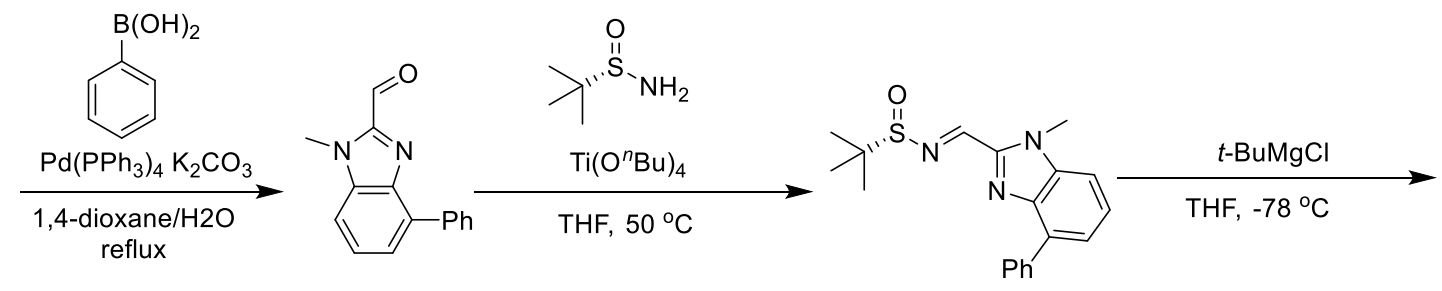

B3

B4<smiles>Cn1c(C(NS(=O)C(C)(C)C)C(C)(C)C)nc2c(-c3ccccc3)cccc21</smiles>

B5<smiles>CO[C@H](C)C(N)c1nc2c(-c3ccccc3)cccc2n1C</smiles>

L9

\section{Procedure for the synthesis of L9 $(\operatorname{method} C)^{3}$}

Synthesis of compound B1: 3-Bromo- $\mathrm{N}^{1}$-methylbenzene-1,2-diamine, $2.01 \mathrm{~g}(10 \mathrm{mmol})$ and 3 equiv. of glycolic acid (2.28 g, $\left.30 \mathrm{mmol}\right)$ were dissolved in $30 \mathrm{~mL}$ of a solution of hydrochloric acid $(4 \mathrm{~N})$, then the mixtures were refluxed for 6 hours. After cooling to room temperature, $5 \%$ $\mathrm{NaHCO}_{3}$ was added slowly to adjust the $\mathrm{pH}$ to around 9 . The resulting precipitate was then isolated by filtration, and it was washed with water and dried to obtain (4-bromo-1-methyl-1H-benzo[d]imidazol-2-yl) methanol (B1).

\section{(4-bromo-1-methyl-1H-benzo[d]imidazol-2-yl)methanol}

White solid, 70\% isolated yield, ${ }^{1} \mathrm{H}$ NMR $(400 \mathrm{MHz}, \mathrm{MeOD}) \delta 7.53-7.48(\mathrm{~m}, 1 \mathrm{H}), 7.44(\mathrm{dd}, J=8.0,0.8 \mathrm{~Hz}, 1 \mathrm{H}), 7.21(\mathrm{t}, J=8.0 \mathrm{~Hz}, 1 \mathrm{H}), 4.88(\mathrm{~s}$, 1H), 3.92 (s, 1H). ${ }^{13} \mathrm{C}$ NMR (101 MHz, MeOD) $\delta 155.80,141.22,137.99,126.49,125.14,113.19,110.53,57.53,31.00$. HRMS (ESI-MS) [M+H] $:$ calculated for $\left[\mathrm{C}_{9} \mathrm{H}_{10} \mathrm{BrN}_{2} \mathrm{O}\right]^{+}: 240.9971$, found: 240.9979 .

Synthesis of compound B2: The (4-bromo-1-methyl-1H-benzo[d]imidazol-2-yl) methanol (2.34 g, $9.7 \mathrm{mmol}$ ) was dissolved in DCM (40 mL), then DMP $(6.17 \mathrm{~g}, 14.55 \mathrm{mmol})$ was add to the solution slowly. The mixture was stirred for $12 \mathrm{~h}$. Saturated sodium thiosulfate solution was added to the reaction mixture, then the aqueous layer was extracted with DCM and the combined organic layer was dried with $\mathrm{Na}_{2} \mathrm{SO}_{4}$. After the solvent was evaporated, the residue was purified using column chromatography $(\mathrm{PE} / \mathrm{EA}=3 / 1)$ to give the 4-bromo-1-methyl-1H-benzo[d]imidazole-2carbaldehyde (B2). 
White solid, 39\% isolated yield, ${ }^{1} \mathrm{H}$ NMR $\left(400 \mathrm{MHz}, \mathrm{CDCl}_{3}\right) \delta 10.15(\mathrm{~s}, 1 \mathrm{H}), 7.58-7.56(\mathrm{~m}, 1 \mathrm{H}), 7.42-7.40(\mathrm{~m}, 1 \mathrm{H}), 7.36-7.28(\mathrm{~m}, 1 \mathrm{H}), 4.13(\mathrm{~s}$, $3 \mathrm{H}) .{ }^{13} \mathrm{C}$ NMR $\left(100 \mathrm{MHz}, \mathrm{CDCl}_{3}\right) \delta 185.03,146.16,141.45,137.26,127.45,127.06,116.00,110.01,31.85$. HRMS $\left(\right.$ ESI-MS) $[\mathrm{M}+\mathrm{H}]^{+}$: calculated for $\left[\mathrm{C}_{9} \mathrm{H}_{9} \mathrm{BrN}_{2} \mathrm{O}\right]^{+}: 238.9815$, found: 238.9813 .

Synthesis of compound B3: 4-Bromo-1-methyl-1H-benzo[d]imidazole-2-carbaldehyde. (359 mg, $1.5 \mathrm{mmol}$ ), phenylboronic acid (238 mg, 1.95 $\mathrm{mmol}), \mathrm{K}_{2} \mathrm{CO}_{3}(311 \mathrm{mg}, 2.25 \mathrm{mmol})$, and $\mathrm{Pd}\left(\mathrm{PPh}_{3}\right) 4(87 \mathrm{mg}, 0.075 \mathrm{mmol})$ were added into a $100 \mathrm{~mL}$ Schlenk tube. A mixture of 1,4-dioxane (12 $\mathrm{mL})$ and $\mathrm{H}_{2} \mathrm{O}(3 \mathrm{~mL})$ was added into the Schlenk tube under Ar atmosphere. The reaction mixture was heated to reflux at $100{ }^{\circ} \mathrm{C}$ for $12 \mathrm{~h}$. After cooling to room temperature, the solvent was evaporated under reduced pressure and the residue was diluted with $\mathrm{H}_{2} \mathrm{O}$, extracted with $\mathrm{CH}_{2} \mathrm{Cl}_{2}$. The combined organic layer was washed with brine, dried over $\mathrm{Na}_{2} \mathrm{SO}_{4}$, and then evaporated to dryness. The crude product was purified by flash column chromatography on silica gel using $\mathrm{PE} / \mathrm{EA}=1 / 5$ as the eluent to give the product as a light yellow solid.

\section{1-methyl-4-phenyl-1H-benzo[d]imidazole-2-carbaldehyde}

Light yellow solid, 90\% isolated yield, ${ }^{1} \mathrm{H}$ NMR $\left(400 \mathrm{MHz}, \mathrm{CDCl}_{3}\right) \delta 10.15(\mathrm{~s}, 1 \mathrm{H}), 8.03-8.00(\mathrm{~m}, 2 \mathrm{H}), 7.57-7.51(\mathrm{~m}, 4 \mathrm{H}), 7.46-7.39(\mathrm{~m}, 2 \mathrm{H}), 4.19$ $(\mathrm{s}, 3 \mathrm{H}) .{ }^{13} \mathrm{C}$ NMR $\left(100 \mathrm{MHz}, \mathrm{CDCl}_{3}\right) \delta 185.48,146.26,140.83,137.71,137.52,135.27,129.29,128.64,128.02,127.07,123.08,109.49,31.47$. HRMS (ESI-MS) $[\mathrm{M}+\mathrm{H}]^{+}$: calculated for $\left[\mathrm{C}_{15} \mathrm{H}_{13} \mathrm{~N}_{2} \mathrm{O}\right]^{+}: 237.1022$, found: 237.1020 .

Synthesis of compound $\mathbf{B} 4: \mathrm{Ti}\left(\mathrm{O}^{n} \mathrm{Bu}\right)_{4}(1.46 \mathrm{~g}, 4.3 \mathrm{mmol})$ was added into a solution of aldehyde $\mathbf{B 3}(566 \mathrm{mg}$, $2.39 \mathrm{mmol})$, (S)-tertbutanesulfinamidesulfinimines $(290 \mathrm{mg}, 2.39 \mathrm{mmol})$ in $5 \mathrm{~mL}$ THF. The reaction mixture was heated at $50{ }^{\circ} \mathrm{C}$ for $12 \mathrm{~h}$. Then, the reaction mixture was quenched by saturated $\mathrm{NaHCO}_{3}$ solution, the mixture was filtered by celite and washed with EA for twice. The filtrate was extracted with EA, dried over anhydrous $\mathrm{Na}_{2} \mathrm{SO}_{4}$, and then the solvent was removed under reduced pressure. The crude product was purified by column chromatography $(\mathrm{PE} / \mathrm{EA}=1: 3)$ to give the product as a white solid.

(S,E)-2-methyl-N-((1-methyl-4-phenyl-1H-benzo[d]imidazol-2-yl)methylene)propane-2-sulfinamide<smiles>Cn1c(/C=N/S(=O)C(C)(C)C)nc2c(-c3ccccc3)cccc21</smiles>

White solid, $93 \%$ isolated yield, [a] $]_{D}^{20}=+316\left(\mathrm{c} 0.188\right.$ in $\left.\mathrm{CHCl}_{3}\right) .{ }^{1} \mathrm{H}$ NMR $\left(400 \mathrm{MHz}, \mathrm{CDCl}_{3}\right) \delta 8.94(\mathrm{~s}, 1 \mathrm{H}), 8.04-8.02(\mathrm{~m}, 2 \mathrm{H}), 7.54-7.49(\mathrm{~m}$, 4H), 7.45-7.38 (m, 2H), $4.22(\mathrm{~s}, 3 \mathrm{H}), 1.30(\mathrm{~s}, 9 \mathrm{H}) .{ }^{13} \mathrm{C} \mathrm{NMR}\left(100 \mathrm{MHz}, \mathrm{CDCl}_{3}\right) \delta 155.96,145.91,140.79,137.82,137.57,134.37,129.29,128.61$, 127.90, 126.03, 122.70, 109.01, 58.08, 32.28, 22.64. HRMS (ESI-MS) $[\mathrm{M}+\mathrm{H}]^{+}$: calculated for $\left[\mathrm{C}_{19} \mathrm{H}_{22} \mathrm{~N}_{3} \mathrm{OS}\right]^{+}: 340.1478$, found: 340.1478 .

Synthesis of compound B5: To a solution of $\mathbf{B} 4\left(339 \mathrm{mg}, 0.25 \mathrm{M}\right.$ in dry THF) at $-78{ }^{\circ} \mathrm{C}$ for $10 \mathrm{~min}$ under Ar, was added Grignard reagent (4.0 equiv., $1 \mathrm{M}$ in dry THF). The mixture was stirred at $-78^{\circ} \mathrm{C}$ for $1 \mathrm{~h}$, then warmed to room temperature with stirring overnight. When reaction was completed, the mixture was quenched by saturated aqueous solution of $\mathrm{NH}_{4} \mathrm{Cl}$, and extracted with $\mathrm{EA}$. The extracts were washed by saturated salt water and dried over anhydrous $\mathrm{Na}_{2} \mathrm{SO}_{4}$, the solvent was removed under reduced pressure. The crude product was purified by column chromatography $(\mathrm{PE} / \mathrm{EA}=1: 2)$ to give the product as a white solid.

\section{(S)-N-((S)-2,2-dimethyl-1-(1-methyl-4-phenyl-1H-benzo[d]imidazol-2-yl)propyl)-2-methylpropane-2-sulfinamide}<smiles>Cn1c([C@H](NS(=O)C(C)(C)C)C(C)(C)C)nc2c(-c3ccccc3)cccc21</smiles>

White solid, 23\% isolated yield, [a] $]_{\boldsymbol{D}}^{20}=-15.5\left(\mathrm{c} 0.220\right.$ in $\left.\mathrm{CHCl}_{3}\right) .{ }^{1} \mathrm{H} \mathrm{NMR}\left(400 \mathrm{MHz}, \mathrm{CDCl}_{3}\right) \delta 8.07(\mathrm{~d}, J=8.0 \mathrm{~Hz}, 2 \mathrm{H}), 7.48-7.42(\mathrm{~m}, 3 \mathrm{H}), 7.36-$ 7.27 (m, 3H), $4.74(\mathrm{~d}, J=8.0 \mathrm{~Hz}, 1 \mathrm{H}), 4.26(\mathrm{~d}, J=8.0 \mathrm{~Hz}, 1 \mathrm{H}), 3.84(\mathrm{~s}, 3 \mathrm{H}), 1.25(\mathrm{~s}, 9 \mathrm{H}), 1.05(\mathrm{~s}, 9 \mathrm{H}) .{ }^{13} \mathrm{C} \mathrm{NMR}\left(100 \mathrm{MHz}, \mathrm{CDCl}_{3}\right) \delta 154.70$, $140.00,138.66,136.17,131.94,129.39,128.11,127.07,122.67,121.39,108.79,60.09,56.37,37.67,30.61,26.44,22.98$. HRMS (ESI-MS) $[\mathrm{M}+\mathrm{H}]^{+}$: calculated for $\left[\mathrm{C}_{23} \mathrm{H}_{32} \mathrm{~N}_{3} \mathrm{OS}\right]^{+}: 398.2261$, found: 398.2255 .

Synthesis of compound L10: The derived benzimidazole $\mathbf{B 5}(198.79 \mathrm{mg}, 0.5 \mathrm{mmol})$ was dissolved in $\mathrm{CH}_{2} \mathrm{Cl}_{2}(2 \mathrm{~mL})$ and cooled to $0{ }^{\circ} \mathrm{C}$, and $1 \mathrm{~mL}$ hydrogen chloride-1,4-dioxane $(4 \mathrm{M})$ was added slowly into the reaction flask. After $2 \mathrm{~h}$, the solvent was evaporated, and the residue was dissolved in $\mathrm{CH}_{2} \mathrm{Cl}_{2}$. Next, $5 \% \mathrm{NaHCO}_{3}$ was added and the $\mathrm{pH}$ was adjusted to around 9 , then the reaction mixture was extracted with $\mathrm{CH}_{2} \mathrm{Cl}_{2}$. Then the solvent was evaporated, the residue was purified using column chromatography (MeOH/EA=1:5) to give the ligand $\mathbf{L 1 0}$ as a white solid.

(S)-2,2-dimethyl-1-(1-methyl-4-phenyl-1H-benzo[d]imidazol-2-yl)propan-1-amine (L9) 
<smiles>Cn1c(C(N)C(C)(C)C)nc2c(-c3ccccc3)cccc21</smiles>

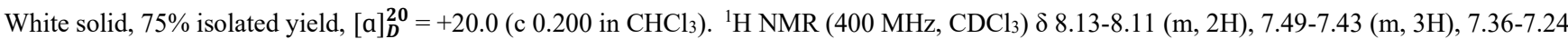
(m, 3H), $3.86(\mathrm{~s}, 1 \mathrm{H}), 3.75$ (s, 3H), 2.07 (s, 2H), 1.05 (s, 9H). ${ }^{13} \mathrm{C}$ NMR (100 MHz, $\left.\mathrm{CDCl}_{3}\right) \delta$ 156.72, 140.09, 138.58, 136.21, 131.72, 129.37, $128.29,127.21,122.32,121.19,108.44,56.72,36.77,30.53,26.49$. HRMS (ESI-MS) $[\mathrm{M}+\mathrm{H}]^{+}$: calculated for $\left[\mathrm{C}_{19} \mathrm{H}_{24} \mathrm{~N}_{3}\right]^{+}: 294.1965$, found: 294.1971

\subsection{Synthesis of Mn complexes Mn1-Mn9}<smiles>CC(=O)OBr</smiles>

L1

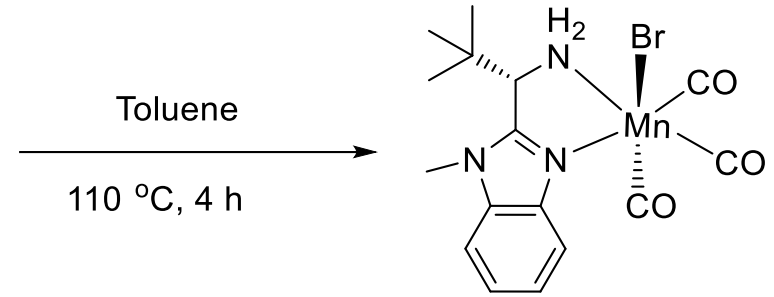

$\mathrm{Mn}-1$

General procedure for the synthesis of complex Mn-1 ${ }^{4}$ : To a solution of ligand L1 (65 mg, $\left.0.3 \mathrm{mmol}\right)$ in toluene was added 1 equiv. of manganese pentacarbonyl bromide $(82 \mathrm{mg}, 0.3 \mathrm{mmol})$. After stirring at $110{ }^{\circ} \mathrm{C}$ during $4 \mathrm{~h}$, the toluene was evaporated under reduced pressure to afford a yellow solid. This solid was washed two times with pentane to give complex, as a yellow powder ( $83 \%$ yield). Crystals suitable for a single-crystal $\mathrm{X}$-ray diffraction study were grown from a solution of $\mathbf{M n - 1}$ in $\mathrm{CH}_{2} \mathrm{Cl}_{2}$ with the slow evaporation of ether.<smiles></smiles>

Mn-1

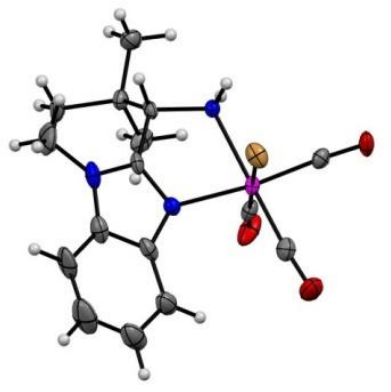

Yellow powder, 83\% isolated yield, $[\mathrm{a}]_{\boldsymbol{D}}^{\mathbf{2 0}}=-353.5(\mathrm{c} 0.2 \mathrm{in} \mathrm{MeOH}),{ }^{1} \mathrm{H}$ NMR $(400 \mathrm{MHz}, \mathrm{DMSO}) \delta 7.95(\mathrm{~d}, J=32.8 \mathrm{~Hz}, 1 \mathrm{H}), 7.64(\mathrm{~d}, J=32.0$ $\mathrm{Hz}, 1 \mathrm{H}), 7.37$ (s, 2H), 4.94 (s, 1H), 4.37 (d, $J=44.4 \mathrm{~Hz}, 2 \mathrm{H}), 3.81$ (s, 3H), 1.05 (s, 9H). ${ }^{13} \mathrm{C}$ NMR (100 MHz, DMSO) $\delta 224.89,223.83,222.12$, $160.63,142.19,137.48,124.04,123.61,118.03,112.07,58.97,38.24,34.42,27.25$. HRMS (ESI-MS) [M-Br] $]^{+}$: calculated for $\left[\mathrm{C}_{16} \mathrm{H}_{19} \mathrm{MnN}_{3} \mathrm{O}_{3}\right]^{+}$: 356.0801, found: 356.0801. IR: 2021, 1922, $1901 \mathrm{~cm}^{-1}$. Anal Calcd for C16H19BrMnN3O3: C, 44.06; H, 4.39; N, 9.63. Found: C, 44.01; H, 4.55; N, 9.30<smiles></smiles>

Yellow powder, 85\% isolated yield, [a] $]_{\boldsymbol{D}}^{\mathbf{2 0}}=-189.5(\mathrm{c} 0.2 \mathrm{in} \mathrm{MeOH}),{ }^{1} \mathrm{H}$ NMR $(400 \mathrm{MHz}$, Acetone) $\delta 8.05(\mathrm{~d}, J=4.8 \mathrm{~Hz}, 1 \mathrm{H}), 7.64(\mathrm{~s}, 1 \mathrm{H}), 7.39$ $(\mathrm{d}, J=21.2 \mathrm{~Hz}, 2 \mathrm{H}), 4.75-4.47(\mathrm{~m}, 5 \mathrm{H}), 1.20(\mathrm{~s}, 12 \mathrm{H}){ }^{13} \mathrm{C}$ NMR $\left(100 \mathrm{MHz}, \mathrm{CD}_{2} \mathrm{Cl}_{2}\right) \delta 222.55,221.63,221.24,158.68,143.36,136.21,124.43$, 124.22, 119.43, 111.89, 60.96, 43.29, 37.93, 27.83, 15.54. HRMS (ESI-MS) [M-Br] $]^{+}$: calculated for $\left[\mathrm{C}_{17} \mathrm{H}_{21} \mathrm{MnN}_{3} \mathrm{O}_{3}\right]^{+}: 370.0958$, found: 370.0957 . IR: 2017, 1914, $1891 \mathrm{~cm}^{-1}$. Anal Calcd for C17H21BrMnN3O3•0.15C7H8•0.4H2O: C, 46.01; H, 4.92; N, 8. 92. Found: C, 46.32; H, 5.08; N, 8.57. 
<smiles></smiles>

Mn-3

Yellow powder, 85\% isolated yield, [a $]_{\boldsymbol{D}}^{\mathbf{2 0}}=-178.5(\mathrm{c} 0.2 \mathrm{in} \mathrm{MeOH}) .{ }^{1} \mathrm{H}$ NMR $(400 \mathrm{MHz}, \mathrm{DMSO}) \delta 8.13(\mathrm{~s}, 1 \mathrm{H}), 7.91(\mathrm{~s}, 1 \mathrm{H}), 7.63(\mathrm{~s}, 1 \mathrm{H}), 7.41$ $(\mathrm{d}, J=28.4 \mathrm{~Hz}, 1 \mathrm{H}), 5.23(\mathrm{~s}, 1 \mathrm{H}), 4.67(\mathrm{~d}, J=51.6 \mathrm{~Hz}, 2 \mathrm{H}), 4.09$ (s, 3H), $1.36(\mathrm{~s}, 3 \mathrm{H}), 1.05(\mathrm{~s}, 3 \mathrm{H}) .{ }^{13} \mathrm{C}$ NMR $(100 \mathrm{MHz}, \mathrm{DMSO}) \delta 223.92$,

223.44, 221.59, 159.65, 140.54, 136.55, 128.81, 123.35, 116.97, 111.35, 56.38, 31.79, 30.82, 18.85, 16.07. HRMS (ESI-MS) [M-Br] $]^{+}$calculated for $\left[\mathrm{C}_{15} \mathrm{H}_{17} \mathrm{MnN}_{3} \mathrm{O}_{3}\right]^{+}: 342.0645$, found: 342.0646 . IR: 2022, 1920, $1905 \mathrm{~cm}^{-1}$. Calcd for C15H17BrMnN3O3•0.4H2O: C, 41.96; H, 4.18; N, 9.79. Found: C, 42.26; H, 4.38; N, 9.58 .

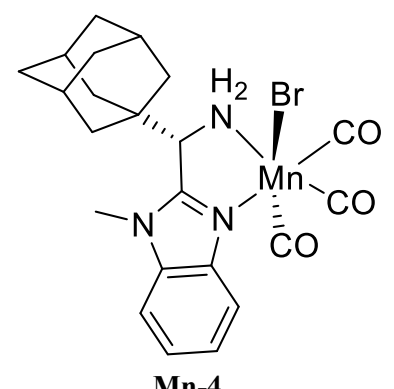

Yellow powder, $84 \%$ isolated yield, $[\mathrm{a}]_{\boldsymbol{D}}^{\mathbf{2 0}}=-236.5(\mathrm{c} 0.2 \mathrm{in} \mathrm{MeOH}) .{ }^{1} \mathrm{H}$ NMR $(400 \mathrm{MHz}, \mathrm{DMSO}) \delta 7.93(\mathrm{~d}, J=34.0 \mathrm{~Hz}, 1 \mathrm{H}), 7.62(\mathrm{~d}, J=32.4$ $\mathrm{Hz}, 1 \mathrm{H}), 7.35(\mathrm{~s}, 1 \mathrm{H}), 7.14(\mathrm{~d}, J=30.8 \mathrm{~Hz}, 1 \mathrm{H}), 5.01(\mathrm{~s}, 1 \mathrm{H}), 4.20(\mathrm{~d}, J=33.2 \mathrm{~Hz}, 2 \mathrm{H}), 3.79(\mathrm{~s}, 3 \mathrm{H}), 1.90(\mathrm{~s}, 3 \mathrm{H}), 1.74(\mathrm{~s}, 3 \mathrm{H}), 1.59(\mathrm{~d}, J=28.8$ $\mathrm{Hz}, 9 \mathrm{H}) .{ }^{13} \mathrm{C}$ NMR (100 MHz, DMSO) $\delta 224.87,223.86,222.18,159.90,142.24,137.41,129.30,123.51,117.99,112.01,59.57,39.97,38.19$, 36.24, 34.46, 28.22. HRMS (ESI-MS) [M-Br] $]^{+}$: calculated for $\left[\mathrm{C}_{22} \mathrm{H}_{25} \mathrm{MnN}_{3} \mathrm{O}_{3}\right]^{+}: 434.1271$, found: 434.1279 . IR: 2021, 1928, $1907 \mathrm{~cm}^{-1}$. Anal Calcd for $\mathrm{C} 22 \mathrm{H} 25 \mathrm{BrMnN} 3 \mathrm{O} 3 \bullet 0.8 \mathrm{H} 2 \mathrm{O}: \mathrm{C}, 49.98 ; \mathrm{H}, 5.07$; N, 7.95. Found: C, 50.09; H, 4.91; N, 7.57.

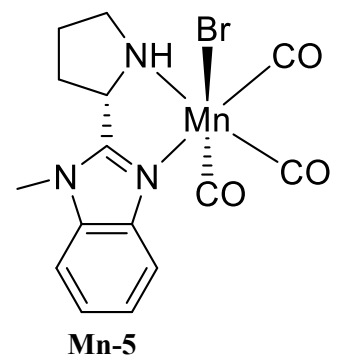

Yellow powder, $85 \%$ isolated yield, $[\mathrm{a}]_{\boldsymbol{D}}^{\mathbf{2 0}}=-163.5(\mathrm{c} 0.2 \mathrm{in} \mathrm{MeOH}),{ }^{1} \mathrm{H} \mathrm{NMR}\left(400 \mathrm{MHz}, \mathrm{CD}_{2} \mathrm{Cl}_{2}\right) \delta 8.07(\mathrm{~s}, 1 \mathrm{H}), 7.44(\mathrm{~s}, 2 \mathrm{H}), 7.21(\mathrm{~d}, J=24.0$ $\mathrm{Hz}, 1 \mathrm{H}), 4.88(\mathrm{~s}, 1 \mathrm{H}), 3.72(\mathrm{~s}, 3 \mathrm{H}), 3.30(\mathrm{~s}, 1 \mathrm{H}), 2.59(\mathrm{~s}, 1 \mathrm{H}), 2.29(\mathrm{~d}, J=42.4 \mathrm{~Hz}, 1 \mathrm{H}), 1.98(\mathrm{~d}, J=48.8 \mathrm{~Hz}, 2 \mathrm{H}), 1.27(\mathrm{~s}, 1 \mathrm{H}), 0.88(\mathrm{~s}, 1 \mathrm{H}) .{ }^{13} \mathrm{C}$ NMR (100 MHz, DMSO) $\delta 223.44,221.20,220.31,159.72,140.18,128.13,123.70,123.44,116.53,111.28,58.42,55.86,31.01,29.82,27.41$. HRMS (ESI-MS) [M-Br] $]^{+}$: calculated for $\left[\mathrm{C}_{15} \mathrm{H}_{15} \mathrm{MnN}_{3} \mathrm{O}_{3}\right]^{+}: 340.0488$, found: 340.0485 . IR: 2020, 1921, $1901 \mathrm{~cm}^{-1}$. Anal Calcd for C15H15BrMnN3O3•0.2C7H8: C, 44.91; H, 3.82; N, 9.58. Found: C, 44.84; H, 3.96; N, 9.12.

Crystals suitable for a single-crystal X-ray diffraction study were grown from a solution of $\mathbf{M n - 6}$ in $\mathrm{CH}_{2} \mathrm{Cl}_{2}$ layered with hexane. 

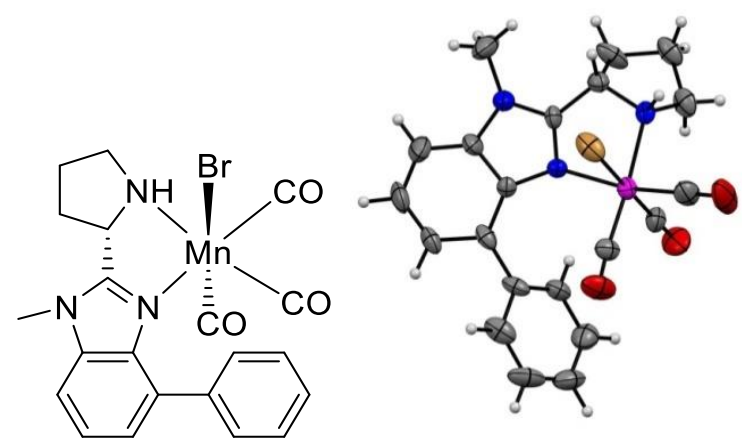

Mn-6

Yellow powder, 81\% isolated yield, [a $]_{\boldsymbol{D}}^{\mathbf{2 0}}=-386.0(\mathrm{c} 0.2 \mathrm{in} \mathrm{MeOH}) .{ }^{1} \mathrm{H}$ NMR $\left(400 \mathrm{MHz}, \mathrm{CD}_{2} \mathrm{Cl}_{2}\right) \delta 7.73(\mathrm{~s}, 2 \mathrm{H}), 7.44(\mathrm{~s}, 6 \mathrm{H}), 4.65(\mathrm{~s}, 1 \mathrm{H}), 3.70$ $(\mathrm{s}, 3 \mathrm{H}), 3.30(\mathrm{~s}, 1 \mathrm{H}), 2.55(\mathrm{~s}, 1 \mathrm{H}), 2.21(\mathrm{~s}, 1 \mathrm{H}), 1.93(\mathrm{~s}, 2 \mathrm{H}), 1.27(\mathrm{~s}, 1 \mathrm{H}), 0.88(\mathrm{~s}, 1 \mathrm{H}) .{ }^{13} \mathrm{C} \mathrm{NMR}(100 \mathrm{MHz}, \mathrm{DMSO}) \delta 222.79,218.07,215.23$, $161.79,138.52$, 137.81, 137.42, 131.60, 130.02, 128.35, 127.48, 125.51, 123.25, 109.69, 58.28, 57.07, 56.11, 30.76, 27.37. HRMS (ESI-MS) [M$\mathrm{Br}]^{+}$: calculated for $\left[\mathrm{C}_{21} \mathrm{H}_{29} \mathrm{MnN}_{3} \mathrm{O}_{3}\right]^{+}: 416.0801$, found: 416.0802. IR: 2024, 1926, $1888 \mathrm{~cm}^{-1}$. Anal Calcd for C16H19BrMnN3O3: C, 44.06; H, 4.39; N, 9.63. Found: C, 44.01; H, 4.54; N, 9.30. Anal Calcd for C21H19BrMnN3O3•0.6H2O: C, 49.74; H, 4.02; N, 8.29. Found: C, 49.68; H, $3.87 ; \mathrm{N}, 7.96$

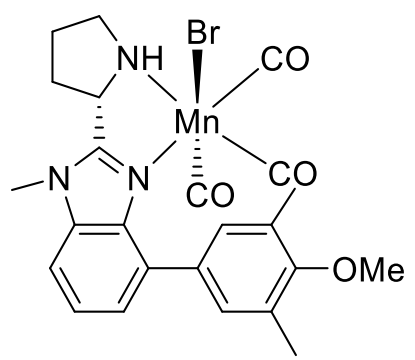

Mn-7

Yellow powder, 81\% isolated yield, [a] $]_{D}^{20}=-258.5\left(\mathrm{c} 0.2\right.$ in MeOH), ${ }^{1} \mathrm{H}$ NMR $(400 \mathrm{MHz}, \mathrm{DMSO}) \delta 6.80(\mathrm{~s}, 5 \mathrm{H}), 4.35(\mathrm{~s}, 1 \mathrm{H}), 3.28(\mathrm{~s}, 6 \mathrm{H}), 1.87(\mathrm{~s}$, $13 \mathrm{H}) .{ }^{13} \mathrm{C}$ NMR (100 MHz, DMSO) $\delta 224.37,222.98,217.67,161.34,156.25,138.38,137.05,133.72,131.84,130.29,128.68,128.01,125.73$,

125.11, 122.77, 109.39, 59.21, 58.18, 56.03, 34.31, 30.72, 26.57, 15.80. HRMS (ESI) [M-Br] $]^{+}$: calculated for $\left[\mathrm{C}_{24} \mathrm{H}_{25} \mathrm{MnN}_{3} \mathrm{O}_{4}\right]^{+}: 474.1220$, found: 474.1209. IR: 2022, 1920, $1905 \mathrm{~cm}^{-1}$. Calcd for C24H25BrMnN3O3•0.15C7H8•0.8H2O: C, 51.65; H, 4.81; N, 7.21. Found: C, 51.42; H, 4.62; N, 6.91 .

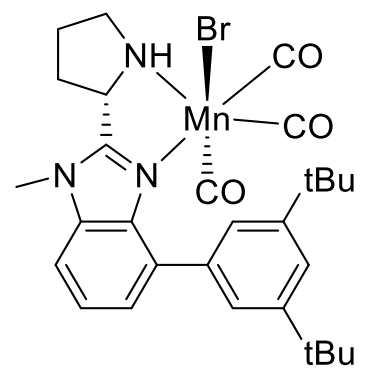

Mn-8

Yellow powder, 81\% isolated yield, [a] $]_{\boldsymbol{D}}^{20}=-222.0(\mathrm{c} 0.2 \mathrm{in} \mathrm{MeOH}),{ }^{1} \mathrm{H} \mathrm{NMR}\left(400 \mathrm{MHz}, \mathrm{CD}_{2} \mathrm{Cl}_{2}\right) \delta 7.82-6.83(\mathrm{~m}, 6 \mathrm{H}), 4.92(\mathrm{~s}, 1 \mathrm{H}), 4.09(\mathrm{~s}, 1 \mathrm{H})$, $3.75(\mathrm{~s}, 3 \mathrm{H}), 3.28(\mathrm{~s}, 1 \mathrm{H}), 2.58(\mathrm{~s}, 1 \mathrm{H}), 2.36(\mathrm{~s}, 1 \mathrm{H}), 2.21(\mathrm{~s}, 1 \mathrm{H}), 2.03(\mathrm{~s}, 2 \mathrm{H}), 1.36(\mathrm{~s}, 18 \mathrm{H}) .{ }^{13} \mathrm{C} \mathrm{NMR}\left(100 \mathrm{MHz}, \mathrm{CD}_{2} \mathrm{Cl}_{2}\right) \delta 225.78,223.20$, $217.78,160.55,151.95,150.08,139.59,138.92,137.68,135.50,128.59,128.51,125.05,124.44,123.65,109.05,60.68,59.26,56.24,35.23,35.22$, 32.30, 32.21, 32.01, 31.55, 31.40, 27.92, 23.08, 14.33. HRMS (ESI) [M-Br] $]^{+}$: calculated for $\left[\mathrm{C}_{29} \mathrm{H}_{35} \mathrm{MnN}_{3} \mathrm{O}_{3}\right]^{+}: 528.2053$, found: 528.2040. IR: 2026, 1932, $1899 \mathrm{~cm}^{-1}$. Calcd for C29H35BrMnN3O3•0.25C7H8: C, 58.49; H, 5.91; N, 6.65. Found: C, 58.26; H, 6.08; N, 6.21. 


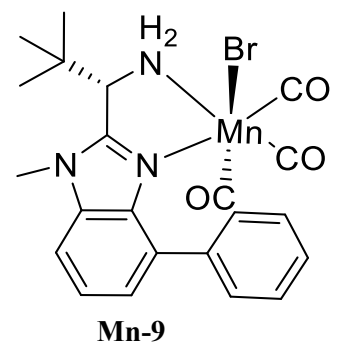

Yellow powder, 82\% isolated yield, [a $]_{D}^{20}=-454.5(\mathrm{c} 0.2 \mathrm{in} \mathrm{MeOH}),{ }^{1} \mathrm{H}$ NMR $(400 \mathrm{MHz}, \mathrm{DMSO}) \delta 8.19-7.00(\mathrm{~m}, 8 \mathrm{H}), 4.74(\mathrm{~s}, 1 \mathrm{H}), 4.38(\mathrm{~d}, J=$ $46.0 \mathrm{~Hz}, 2 \mathrm{H}), 3.79$ (s, 3H), 1.04 (s, 9H). ${ }^{13} \mathrm{C}$ NMR (100 MHz, DMSO) $\delta 225.50,223.57,218.54,161.91,140.39,137.99,137.75,131.07,129.65$, $128.70,128.42,128.16,127.45,124.71,123.33,110.10,57.66,37.60,32.78,26.04$. HRMS (ESI-MS) [M-Br] $]^{+}$calculated for $\left[\mathrm{C}_{22} \mathrm{H}_{23} \mathrm{MnN}_{3} \mathrm{O}_{3}\right]^{+}$: 432.1114, found: 432.1121. IR: 2024, 1927, $1895 \mathrm{~cm}^{-1}$. Calcd for C22H23BrMnN3O3•1.5H2O: C, 49.00; H, 4.86; N, 7.79. Found: C, 49.14; H, $4.44 ; \mathrm{N}, 7.52$.

\section{Reaction conditions optimization for ATH}

\subsection{Table S1. Condition Screening}

\begin{tabular}{|l|l|l|l|l|l|}
\hline Entry & Mn Cat. & Time $[\mathrm{h}]$ & Temp. $\left[{ }^{\circ} \mathrm{C}\right]$ & Yield [\%] & ee [\%] \\
\hline 1 & Mn-1 & 24 & 40 & 93 & 73 \\
\hline 2 & Mn-1 & 24 & 60 & 96 & 68 \\
\hline 3 & Mn-6 & 24 & 40 & 65 & 87 \\
\hline 4 & Mn-6 & 24 & 50 & 87 & 86 \\
\hline 5 & Mn-6 & 24 & 60 & 92 & 83 \\
\hline
\end{tabular}

\subsection{General procedure for ATH using Mn-1 or Mn-6 as catalyst}

A $10 \mathrm{~mL}$ Schlenk tube was charged with catalyst Mn-6 (2.0 mol\% or $5.0 \mathrm{~mol} \%), 2 \mathrm{~mL}$ of $i-\mathrm{PrOH}, \mathrm{KO}^{t} \mathrm{Bu}(4 \mathrm{~mol} \%$ or $10 \mathrm{~mol} \%)$ and a stir bar under argon. Then ketone $(0.5 \mathrm{~mol})$ was added in to the tube, and the mixture was stirred at $50{ }^{\circ} \mathrm{C}$ for $24 \mathrm{~h}\left(50{ }^{\circ} \mathrm{C}\right.$ for $24 \mathrm{~h}$ for catalyst Mn- $1 ; 80{ }^{\circ} \mathrm{C}$ for $24 \mathrm{~h}$ some more bulky substrates). The reaction mixture was purified by silica gel chromatography (PE/EA) to give the desired product. The enantiomeric excess of alcohol was determined by HPLC or GC analysis.

(S)-1-phenylethan-1-ol (2a) ${ }^{5}$<smiles>C[C@H](O)c1ccccc1</smiles>

Colorless oil, $87 \%$ GC yield, [a $]_{D}^{20}=-28.50$ (c 0.200 in $\mathrm{CHCl}_{3}$ ) [lit. ${ }^{5}[\mathrm{a}]_{D}^{25}=+43.43$ (c 1.0 in $\left.\mathrm{CHCl}_{3}\right), 92 \%$ ee, $R$ ], ${ }^{1} \mathrm{H} \mathrm{NMR}\left(400 \mathrm{MHz}, \mathrm{CDCl}_{3}\right) \delta$ $7.36(\mathrm{~d}, J=4.0 \mathrm{~Hz}, 4 \mathrm{H}), 7.29(\mathrm{dd}, J=8.4,4.0 \mathrm{~Hz}, 1 \mathrm{H}), 4.85(\mathrm{q}, J=6.4 \mathrm{~Hz}, 1 \mathrm{H}), 2.58(\mathrm{~s}, 1 \mathrm{H}), 1.48(\mathrm{~d}, J=6.4 \mathrm{~Hz}, 3 \mathrm{H}) .{ }^{13} \mathrm{C} \mathrm{NMR}(100 \mathrm{MHz}$, $\left.\mathrm{CDCl}_{3}\right) \delta 145.90,128.49,127.44,125.46,70.33,25.17$.The ee was determined by Agilent $6820 \mathrm{GC}$ with a CP-Chirasil-Dex CB column using a flame ionization detector (FID) operating at $280{ }^{\circ} \mathrm{C}$. Injector temperature was set at $250{ }^{\circ} \mathrm{C} .\left(80{ }^{\circ} \mathrm{C}\right.$ isothermal for 2 minutes, $5{ }^{\circ} \mathrm{C} / \mathrm{min}, 170{ }^{\circ} \mathrm{C}$ for 5 minutes, $t_{\mathrm{R}}=12.759 \mathrm{~min}$ and $13.321 \mathrm{~min}$ ). $t_{\mathrm{R}}=13.164 \mathrm{~min}$ for minor isomer and $t_{\mathrm{R}}=13.510 \mathrm{~min}$ for major isomer. $86 \%$ ee.

(S)-1-(o-tolyl)ethan-1-ol (2b) ${ }^{5}$<smiles>Cc1ccccc1[C@@H](C)O</smiles> 


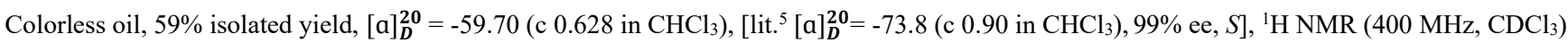
$\delta 7.47(\mathrm{~d}, J=7.2 \mathrm{~Hz}, 1 \mathrm{H}), 7.22-7.09(\mathrm{~m}, 3 \mathrm{H}), 5.07(\mathrm{~d}, J=6.0 \mathrm{~Hz}, 1 \mathrm{H}), 2.31(\mathrm{~s}, 3 \mathrm{H}), 2.11(\mathrm{~s}, 1 \mathrm{H}), 1.42(\mathrm{~d}, J=6.0 \mathrm{~Hz}, 3 \mathrm{H}) .{ }^{13} \mathrm{C} \mathrm{NMR}(100 \mathrm{MHz}$, $\left.\mathrm{CDCl}_{3}\right) \delta 143.89,134.21,130.36,127.15,126.37,124.52,66.76,23.93,18.92$. The optical purity was determined by Agilent $6820 \mathrm{GC}$ with a CPChirasil-Dex CB column using a flame ionization detector (FID) operating at $280{ }^{\circ} \mathrm{C}$. Injector temperature was set at $250{ }^{\circ} \mathrm{C} .\left(80^{\circ} \mathrm{C}\right.$ isothermal for 2 minutes, $5{ }^{\circ} \mathrm{C} / \mathrm{min}, 170^{\circ} \mathrm{C}$ for 5 minutes, $\mathrm{t}_{\mathrm{R}}=16.346 \mathrm{~min}$ and $17.417 \mathrm{~min}$ ). $t_{\mathrm{R}}=16.573 \mathrm{~min}$ for minor isomer and $t_{\mathrm{R}}=17.412 \mathrm{~min}$ for major isomer. $93 \%$ ee.

(S)-1-(2-methoxyphenyl)ethan-1-ol (2c) ${ }^{5}$<smiles>COc1ccccc1[C@@H](C)O</smiles>

Colorless oil, 94\% isolated yield, [a] $]_{\boldsymbol{D}}^{\mathbf{2 0}}=-20.79\left(\mathrm{c} 1.270\right.$ in $\left.\mathrm{CHCl}_{3}\right),\left[\right.$ lit. ${ }^{5}[\mathrm{a}]_{\boldsymbol{D}}^{25}=+22.10\left(\mathrm{c} 1.0\right.$ in $\left.\mathrm{CHCl}_{3}\right), 90 \%$ ee, R], ${ }^{1} \mathrm{H} \mathrm{NMR}(400 \mathrm{MHz}, \mathrm{CDCl}$ ) $\delta 7.33(\mathrm{~d}, J=7.2 \mathrm{~Hz}, 1 \mathrm{H}), 7.25-7.21(\mathrm{~m}, 1 \mathrm{H}), 6.95(\mathrm{t}, J=7.6 \mathrm{~Hz}, 1 \mathrm{H}), 6.86(\mathrm{~d}, J=8.0 \mathrm{~Hz}, 1 \mathrm{H}), 5.08(\mathrm{dd}, J=12.4,6.0 \mathrm{~Hz}, 1 \mathrm{H}), 3.83(\mathrm{~s}, 3 \mathrm{H}), 2.71$ $(\mathrm{s}, 1 \mathrm{H}), 1.48(\mathrm{~d}, J=6.0 \mathrm{~Hz}, 3 \mathrm{H}) .{ }^{13} \mathrm{C}$ NMR $\left(100 \mathrm{MHz}, \mathrm{CDCl}_{3}\right) \delta 156.53,133.55,128.28,126.10,120.81,110.44,66.40,55.27,22.95$. HPLCseparation conditions: Chiralcel OD-H, $254 \mathrm{~nm}$, hexane : ${ }^{i} \mathrm{PrOH}=99: 1$; flow rate $1.0 \mathrm{~mL} / \mathrm{min} ; 30{ }^{\circ} \mathrm{C}, \mathrm{t}_{1}=35.997 \mathrm{~min}, \mathrm{t}_{2}=38.907 \mathrm{~min}, \mathrm{t}_{\mathrm{R}}=36.316$ $\min$ (major), $t_{R}=40.221 \min$ (minor). $91 \%$ ee.

(S)-1-(2-bromophenyl)ethan-1-ol (2d) ${ }^{6}$<smiles>C[C@@H](O)c1ccccc1Br</smiles>

Colorless oil, 95\% isolated yield, [a] $]_{\boldsymbol{D}}^{20}=-46.20$ (c 1.000 in $\left.\mathrm{CHCl}_{3}\right),\left[\mathrm{lit}^{6}{ }^{6}[\mathrm{a}]_{\boldsymbol{D}}^{\mathbf{2 0}}=-54.5\left(\mathrm{c} 1.65\right.\right.$ in $\left.\mathrm{CHCl}_{3}\right), 99 \%$ ee, $\left.\mathrm{S}\right] .{ }^{1} \mathrm{H} \mathrm{NMR}\left(400 \mathrm{MHz}, \mathrm{CDCl}_{3}\right)$ $\delta 7.54(\mathrm{~d}, J=8.0 \mathrm{~Hz}, 1 \mathrm{H}), 7.48(\mathrm{~d}, J=8.0 \mathrm{~Hz}, 1 \mathrm{H}), 7.30(\mathrm{t}, J=8.0 \mathrm{~Hz}, 1 \mathrm{H}), 7.09(\mathrm{t}, J=8.0 \mathrm{~Hz}, 1 \mathrm{H}), 5.19(\mathrm{q}, J=6.4 \mathrm{~Hz}, 1 \mathrm{H}), 2.53(\mathrm{~s}, 1 \mathrm{H}), 1.44$ $(\mathrm{dd}, J=6.4,0.8 \mathrm{~Hz}, 3 \mathrm{H}) .{ }^{13} \mathrm{C} \mathrm{NMR}\left(100 \mathrm{MHz}, \mathrm{CDCl}_{3}\right) \delta 144.67,132.63,128.72,127.84,126.69,121.67,69.13$, 23.59. HPLC-separation conditions: Chiralcel OD-H, $254 \mathrm{~nm}$, hexane : ${ }^{i} \mathrm{PrOH}=99: 1$; flow rate $1.0 \mathrm{~mL} / \mathrm{min} ; 30{ }^{\circ} \mathrm{C}, \mathrm{t}_{1}=26.606 \mathrm{~min}, \mathrm{t}_{2}=29.516 \mathrm{~min}, \mathrm{t}_{\mathrm{R}}=27.686 \mathrm{~min}$ (minor), $\mathrm{t}_{\mathrm{R}}=30.033 \mathrm{~min}$ (major). $90 \%$ ee.

(S)-1-(4-bromophenyl)ethan-1-ol (2e) ${ }^{6}$<smiles>C[C@H](O)c1ccc(Br)cc1</smiles>

Colorless oil, 95\% isolated yield, [a] $]_{\boldsymbol{D}}^{20}=-27.44$ (c 0.430 in $\left.\mathrm{CHCl}_{3}\right),\left[\right.$ lit. ${ }^{6}[\mathrm{a}]_{\boldsymbol{D}}^{20}=-72.1$ (c 1.66 in $\left.\mathrm{CHCl}_{3}\right), 96 \%$ ee, $\left.\mathrm{S}\right] .{ }^{1} \mathrm{H} \mathrm{NMR}\left(400 \mathrm{MHz}, \mathrm{CDCl}_{3}\right)$ $\delta 7.47(\mathrm{~d}, J=8.0 \mathrm{~Hz}, 2 \mathrm{H}), 7.24(\mathrm{~d}, J=8.4 \mathrm{~Hz}, 2 \mathrm{H}), 4.86(\mathrm{q}, J=6.4 \mathrm{~Hz}, 1 \mathrm{H}), 1.89(\mathrm{~s}, 1 \mathrm{H}), 1.47(\mathrm{~d}, J=6.4 \mathrm{~Hz}, 3 \mathrm{H}) .{ }^{13} \mathrm{C} \mathrm{NMR}\left(100 \mathrm{MHz}, \mathrm{CDCl}_{3}\right) \delta$ 144.79, 131.57, 127.16, 121.17, 69.79, 25.27. HPLC-separation conditions: Chiralcel OD-H, $254 \mathrm{~nm}$, hexane : ${ }^{i} \operatorname{PrOH}=98: 2$; flow rate 1.0 $\mathrm{mL} / \mathrm{min} ; 30^{\circ} \mathrm{C}, \mathrm{t}_{1}=20.471 \mathrm{~min}, \mathrm{t}_{2}=22.615 \mathrm{~min}, \mathrm{t}_{\mathrm{R}}=20.213 \min$ (major), $\mathrm{t}_{\mathrm{R}}=22.365 \min$ (minor). $80 \%$ ee.

(S)-1-(4-iodophenyl)ethan-1-ol (2f) ${ }^{6}$<smiles>C[C@H](O)c1ccc(I)cc1</smiles>

Light yellow solid, $98 \%$ isolated yield, $[\mathrm{a}]_{\boldsymbol{D}}^{\mathbf{2 0}}=-30.20$ (c 1.000 in $\left.\mathrm{CHCl}_{3}\right),\left[\mathrm{lit} .{ }^{6}[\mathrm{a}]_{\boldsymbol{D}}^{\mathbf{2 0}}=-33.1\right.$ (c 1.25 in $\left.\mathrm{CHCl}_{3}\right), 96 \%$ ee, $\left.\mathrm{S}\right] .{ }^{1} \mathrm{H} \mathrm{NMR}(400 \mathrm{MHz}$, $\left.\mathrm{CDCl}_{3}\right) \delta 7.62(\mathrm{~d}, J=8.4 \mathrm{~Hz}, 2 \mathrm{H}), 7.04(\mathrm{~d}, J=8.4 \mathrm{~Hz}, 2 \mathrm{H}), 4.73(\mathrm{q}, J=6.4 \mathrm{~Hz}, 1 \mathrm{H}), 2.92(\mathrm{~s}, 1 \mathrm{H}), 1.39(\mathrm{~d}, J=6.4 \mathrm{~Hz}, 3 \mathrm{H}) .{ }^{13} \mathrm{C} \mathrm{NMR}(100 \mathrm{MHz}$, $\left.\mathrm{CDCl}_{3}\right) \delta 145.46,137.49,127.49,92.77,69.70,25.24$. The optical purity was determined by Agilent $6820 \mathrm{GC}$ with a CP-Chirasil-Dex CB column using a flame ionization detector (FID) operating at $280{ }^{\circ} \mathrm{C}$. Injector temperature was set at $250{ }^{\circ} \mathrm{C}$. $\left(80{ }^{\circ} \mathrm{C}\right.$ isothermal for $2 \mathrm{minutes}, 10{ }^{\circ} \mathrm{C} / \mathrm{min}$, $150^{\circ} \mathrm{C}$ for 5 minutes, $0.5^{\circ} \mathrm{C} / \mathrm{min}, 170{ }^{\circ} \mathrm{C}$ for 5 minutes, $\mathrm{t}_{\mathrm{R}}=19.719 \mathrm{~min}$ and $20.597 \mathrm{~min}$ ). $t_{\mathrm{R}}=19.767 \mathrm{~min}$ for minor isomer and $t_{\mathrm{R}}=20.407 \mathrm{~min}$ for major isomer. $88 \%$ ee.

(S)-1-(2-chlorophenyl)ethan-1-ol (2g) ${ }^{6}$ 
<smiles>CC(O)c1ccccc1Cl</smiles>

Colorless oil, $95 \%$ isolated yield, $[\mathrm{a}]_{\boldsymbol{D}}^{\mathbf{2 0}}=-51.00\left(\mathrm{c} 1.000\right.$ in $\left.\mathrm{CHCl}_{3}\right),\left[\mathrm{lit}^{6}[\mathrm{a}]_{\boldsymbol{D}}^{\mathbf{2 0}}=-63.6\left(\mathrm{c} 0.91\right.\right.$ in $\left.\mathrm{CHCl}_{3}\right), 97 \%$ ee, $\left.\mathrm{S}\right] .{ }^{1} \mathrm{H} \mathrm{NMR}\left(400 \mathrm{MHz}, \mathrm{CDCl}_{3}\right)$ $\delta 7.57(\mathrm{dd}, J=8.0,1.2 \mathrm{~Hz}, 1 \mathrm{H}), 7.36-7.23(\mathrm{~m}, 2 \mathrm{H}), 7.18(\mathrm{td}, J=7.6,1.6 \mathrm{~Hz}, 1 \mathrm{H}), 5.26(\mathrm{q}, J=6.4 \mathrm{~Hz}, 1 \mathrm{H}), 2.27(\mathrm{~s}, 1 \mathrm{H}), 1.47(\mathrm{~d}, J=6.4 \mathrm{~Hz}, 3 \mathrm{H})$. ${ }^{13} \mathrm{C}$ NMR $\left(100 \mathrm{MHz}, \mathrm{CDCl}_{3}\right) \delta 143.09,131.63,129.40,128.39,127.22,126.43,66.94,23.52$. The optical purity was determined by Agilent 6820 $\mathrm{GC}$ with a CP-Chirasil-Dex CB column using a flame ionization detector (FID) operating at $280^{\circ} \mathrm{C}$. Injector temperature was set at $250{ }^{\circ} \mathrm{C}$. $\left(80{ }^{\circ} \mathrm{C}\right.$ isothermal for 2 minutes, $5{ }^{\circ} \mathrm{C} / \mathrm{min}, 170^{\circ} \mathrm{C}$ for $5 \mathrm{~min}, \mathrm{t}_{\mathrm{R}}=18.760 \mathrm{~min}$ and $19.909 \mathrm{~min}$ ). $t_{\mathrm{R}}=18.956 \mathrm{~min}$ for minor isomer and $t_{\mathrm{R}}=20.011 \mathrm{~min}$ for major isomer. $87 \%$ ee.

(S)-1-(2-fluorophenyl)ethan-1-ol (2h) ${ }^{7}$<smiles>C[C@H](O)c1ccccc1F</smiles>

Colorless oil, $89 \%$ isolated yield, [a] $]_{\boldsymbol{D}}^{20}=-40.34$ (c 0.932 in $\left.\mathrm{CHCl}_{3}\right),\left[\right.$ lit. ${ }^{7}[\mathrm{a}]_{\boldsymbol{D}}^{20}=+43.6\left(\mathrm{c} 0.95\right.$ in $\left.\mathrm{CHCl}_{3}\right), 95 \%$ ee, R]. ${ }^{1} \mathrm{H} \mathrm{NMR}\left(400 \mathrm{MHz}, \mathrm{CDCl}_{3}\right)$ $\delta 7.46(\mathrm{td}, J=7.6,1.6 \mathrm{~Hz}, 1 \mathrm{H}), 7.25-7.19(\mathrm{~m}, 1 \mathrm{H}), 7.13(\mathrm{td}, J=7.6,0.8 \mathrm{~Hz}, 1 \mathrm{H}), 7.02-6.97(\mathrm{~m}, 1 \mathrm{H}), 5.16(\mathrm{q}, J=6.4 \mathrm{~Hz}, 1 \mathrm{H}), 2.52(\mathrm{~s}, 1 \mathrm{H}), 1.48$ $(\mathrm{d}, J=6.4 \mathrm{~Hz}, 3 \mathrm{H}) .{ }^{13} \mathrm{C} \mathrm{NMR}\left(100 \mathrm{MHz}, \mathrm{CDCl}_{3}\right) \delta 158.18(\mathrm{~d}, J=239.4 \mathrm{~Hz}), 132.68(\mathrm{~d}, J=13.2 \mathrm{~Hz}), 128.73(\mathrm{~d}, J=8.2 \mathrm{~Hz}), 126.64(\mathrm{~d}, J=4.7$ $\mathrm{Hz}), 124.31(\mathrm{~d}, J=3.4 \mathrm{~Hz}), 115.26(\mathrm{~d}, J=21.8 \mathrm{~Hz}), 64.40(\mathrm{~d}, J=3.0 \mathrm{~Hz}), 24.00(\mathrm{~s}) .{ }^{19} \mathrm{~F}$ NMR $\left(376 \mathrm{MHz}, \mathrm{CDCl}_{3}\right) \delta-120.06$. The optical purity was determined by Agilent $6820 \mathrm{GC}$ with a CP-Chirasil-Dex CB column using a flame ionization detector (FID) operating at $280{ }^{\circ} \mathrm{C}$. Injector temperature was set at $250{ }^{\circ} \mathrm{C}$. $\left(80{ }^{\circ} \mathrm{C}\right.$ isothermal for 2 minutes, $2{ }^{\circ} \mathrm{C} / \mathrm{min}, 170{ }^{\circ} \mathrm{C}$ for 5 minutes, $\mathrm{t}_{\mathrm{R}}=18.701 \mathrm{~min}$ and $\left.17.417 \mathrm{~min}\right) . t_{\mathrm{R}}=19.307 \mathrm{~min}$ for minor isomer and $t_{\mathrm{R}}=20.207 \mathrm{~min}$ for major isomer. $87 \%$ ee.

(S)-1-(4-fluorophenyl)ethan-1-ol (2i) ${ }^{6}$<smiles>C[C@H](O)c1ccc(F)cc1</smiles>

Colorless oil, $90 \%$ isolated yield, [a] $]_{D}^{20}=-37.27$ (c 0.432 in $\left.\mathrm{CHCl}_{3}\right)$, [lit. ${ }^{6}[\mathrm{a}]_{\boldsymbol{D}}^{\mathbf{2 0}}=-81.5\left(\mathrm{c} 1.58\right.$ in $\left.\mathrm{CHCl}_{3}\right), 96 \%$ ee, $\left.\mathrm{S}\right] .{ }^{1} \mathrm{H} \mathrm{NMR}(400 \mathrm{MHz}, \mathrm{CDCl} 3)$ $\delta 7.35-7.27(\mathrm{~m}, 2 \mathrm{H}), 7.06-6.97(\mathrm{~m}, 2 \mathrm{H}), 4.85(\mathrm{q}, J=6.4 \mathrm{~Hz}, 1 \mathrm{H}), 2.25(\mathrm{~s}, 1 \mathrm{H}), 1.45(\mathrm{~d}, J=6.5 \mathrm{~Hz}, 3 \mathrm{H}) .{ }^{13} \mathrm{C} \mathrm{NMR}\left(100 \mathrm{MHz}, \mathrm{CDCl}_{3}\right) \delta 162.09(\mathrm{~d}$, $J=243.6 \mathrm{~Hz}), 141.53$ (d, $J=3.0 \mathrm{~Hz}), 127.06$ (d, $J=8.0 \mathrm{~Hz}), 115.25$ (d, $J=21.1 \mathrm{~Hz}), 69.74$ (s), 25.28 (s). ${ }^{19} \mathrm{~F} \mathrm{NMR}\left(376 \mathrm{MHz}, \mathrm{CDCl}_{3}\right) \delta-115.34$. The optical purity was determined by Agilent $6820 \mathrm{GC}$ with a CP-Chirasil-Dex CB column using a flame ionization detector (FID) operating at $280{ }^{\circ} \mathrm{C}$. Injector temperature was set at $250{ }^{\circ} \mathrm{C}$. $\left(80^{\circ} \mathrm{C}\right.$ isothermal for $2 \mathrm{~min}, 2^{\circ} \mathrm{C} / \mathrm{min}, 170{ }^{\circ} \mathrm{C}$ for 5 minutes, $\mathrm{t}_{\mathrm{R}}=19.672 \mathrm{~min}$ and $\left.21.109 \mathrm{~min}\right) . t_{\mathrm{R}}=$ $19.836 \mathrm{~min}$ for minor isomer and $t_{\mathrm{R}}=20.910 \mathrm{~min}$ for major isomer. $81 \%$ ee.

(S)-1-(4-(trifluoromethyl)phenyl)ethan-1-ol (2j) ${ }^{6}$<smiles>C[C@H](O)c1ccc(C(F)(F)F)cc1</smiles>

Colorless oil, 91\% isolated yield, [a] $]_{\boldsymbol{D}}^{\mathbf{2 0}}=-27.48$ (c 0.906 in $\left.\mathrm{CHCl}_{3}\right)$, [lit. ${ }^{6}[\mathrm{a}]_{\boldsymbol{D}}^{\mathbf{2 0}}=-32.0$ (c 0.86 in $\left.\mathrm{CHCl}_{3}\right), 91 \%$ ee, $\left.\mathrm{S}\right] .{ }^{1} \mathrm{H} \mathrm{NMR}\left(400 \mathrm{MHz}, \mathrm{CDCl}_{3}\right)$ $\delta 7.57(\mathrm{~d}, J=8.1 \mathrm{~Hz}, 2 \mathrm{H}), 7.41(\mathrm{~d}, J=8.1 \mathrm{~Hz}, 2 \mathrm{H}), 4.87(\mathrm{q}, J=6.4 \mathrm{~Hz}, 1 \mathrm{H}), 3.06(\mathrm{~s}, 1 \mathrm{H}), 1.44(\mathrm{~d}, J=6.4 \mathrm{~Hz}, 3 \mathrm{H}) .{ }^{13} \mathrm{C} \mathrm{NMR}\left(100 \mathrm{MHz}, \mathrm{CDCl}_{3}\right) \delta$ $149.69(\mathrm{~s}), 129.55$ (q, $J=32.1 \mathrm{~Hz}), 125.64(\mathrm{~s}), 125.37$ (q, $J=3.7 \mathrm{~Hz}), 122.84(\mathrm{~s}), 69.71(\mathrm{~s}), 25.22(\mathrm{~s}) .{ }^{19} \mathrm{~F} \mathrm{NMR}\left(376 \mathrm{MHz}, \mathrm{CDCl}_{3}\right) \delta-62.47 . \mathrm{The}$ optical purity was determined by Agilent $6820 \mathrm{GC}$ with a CP-Chirasil-Dex CB column using a flame ionization detector (FID) operating at $280{ }^{\circ} \mathrm{C}$. Injector temperature was set at $250{ }^{\circ} \mathrm{C}$. $\left(80{ }^{\circ} \mathrm{C}\right.$ isothermal for 2 minutes, $5{ }^{\circ} \mathrm{C} / \mathrm{min}, 170{ }^{\circ} \mathrm{C}$ for 5 minutes, $\mathrm{t}_{\mathrm{R}}=22.391 \mathrm{~min}$ and $\left.24.237 \mathrm{~min}\right)$. $t_{\mathrm{R}}=$ $22.676 \mathrm{~min}$ for minor isomer and $t_{\mathrm{R}}=24.149 \mathrm{~min}$ for major isomer. $86 \%$ ee.

(S)-1-(2-(trifluoromethyl)phenyl)ethan-1-ol (4k) ${ }^{6}$<smiles>C[C@H](O)c1ccccc1C(F)(F)F</smiles> 
Colorless oil, $91 \%$ isolated yield, [a] $]_{D}^{20}=-41.36\left(\mathrm{c} 0.648\right.$ in $\left.\mathrm{CHCl}_{3}\right),\left[\mathrm{lit}^{6}{ }^{6}[\mathrm{a}]_{\boldsymbol{D}}^{20}=-39.1\right.$ (c 1.44 in $\left.\mathrm{MeOH}\right), 96 \%$ ee, $\left.\mathrm{S}\right] .{ }^{1} \mathrm{H} \mathrm{NMR}\left(400 \mathrm{MHz}, \mathrm{CDCl}_{3}\right)$ $\delta 7.81(\mathrm{~d}, J=8.0 \mathrm{~Hz}, 1 \mathrm{H}), 7.65-7.53(\mathrm{~m}, 2 \mathrm{H}), 7.36(\mathrm{t}, J=7.6 \mathrm{~Hz}, 1 \mathrm{H}), 5.34-5.29(\mathrm{~m}, 1 \mathrm{H}), 2.19(\mathrm{~s}, 1 \mathrm{H}), 1.47(\mathrm{~d}, J=7.6 \mathrm{~Hz}, 3 \mathrm{H}) .{ }^{13} \mathrm{C} \mathrm{NMR}(100$ $\left.\mathrm{MHz}, \mathrm{CDCl}_{3}\right) \delta 145.05(\mathrm{~s}), 132.39$ (s), $127.34(\mathrm{~s}), 127.10$ (s), 125.74 (s), 125.29 (q, $\left.J=5.8 \mathrm{~Hz}\right), 123.02(\mathrm{~s}), 65.67(\mathrm{q}, J=2.3 \mathrm{~Hz}), 25.41(\mathrm{~s}) .{ }^{19} \mathrm{~F}$ NMR $\left(376 \mathrm{MHz}, \mathrm{CDCl}_{3}\right) \delta-58.33$. The optical purity was determined by Agilent $6820 \mathrm{GC}$ with a CP-Chirasil-Dex CB column using a flame ionization detector (FID) operating at $280{ }^{\circ} \mathrm{C}$. Injector temperature was set at $250{ }^{\circ} \mathrm{C}$. $\left(80{ }^{\circ} \mathrm{C}\right.$ isothermal for 2 minutes, $2^{\circ} \mathrm{C} / \mathrm{min}, 170{ }^{\circ} \mathrm{C}$ for 5 minutes, $t_{\mathrm{R}}=19.637 \mathrm{~min}$ and $20.993 \mathrm{~min}$ ). $t_{\mathrm{R}}=19.896 \mathrm{~min}$ for minor isomer and $t_{\mathrm{R}}=20.874 \mathrm{~min}$ for major isomer. $86 \%$ ee.

(S)-4-(1-hydroxyethyl)benzonitrile (2l) ${ }^{6}$<smiles>C[C@H](O)c1ccc(C#N)cc1</smiles>

Colorless oil, $80 \%$ isolated yield, [a] $]_{\boldsymbol{D}}^{\mathbf{2 0}}=-30.40\left(\mathrm{c} 0.192\right.$ in $\left.\mathrm{CHCl}_{3}\right)$, [lit. ${ }^{6}[\mathrm{a}]_{\boldsymbol{D}}^{\mathbf{2 0}}=-46.4$ (c 2.40 in $\left.\mathrm{CHCl}_{3}\right), 96 \%$ ee, $\left.\mathrm{S}\right] .{ }^{1} \mathrm{H} \mathrm{NMR}\left(400 \mathrm{MHz}, \mathrm{CDCl}_{3}\right)$ $\delta 7.64-7.56(\mathrm{~m}, 2 \mathrm{H}), 7.46(\mathrm{~d}, J=8.4 \mathrm{~Hz}, 2 \mathrm{H}), 4.93(\mathrm{q}, J=6.4 \mathrm{~Hz}, 1 \mathrm{H}), 2.51(\mathrm{~s}, 1 \mathrm{H}), 1.46(\mathrm{~d}, J=6.4 \mathrm{~Hz}, 3 \mathrm{H}) .{ }^{13} \mathrm{C} \mathrm{NMR}\left(100 \mathrm{MHz}, \mathrm{CDCl}_{3}\right) \delta$ $151.25,132.33,126.10,118.90,110.89,69.58,25.37$. The optical purity was determined by Agilent $6820 \mathrm{GC}$ with a CP-Chirasil-Dex CB column using a flame ionization detector (FID) operating at $280^{\circ} \mathrm{C}$. HPLC-separation conditions: Chiralcel OD-H, $254 \mathrm{~nm}$, hexane : ${ }^{i} \operatorname{PrOH}=98: 2 ;$ flow rate $=1.0 \mathrm{~mL} / \mathrm{min} ; \mathrm{t}_{1}=18.311 \mathrm{~min}, \mathrm{t}_{2}=19.410 \mathrm{~min}, \mathrm{t}_{\mathrm{R}}=17.495 \mathrm{~min}$ (major), $\mathrm{t}_{\mathrm{R}}=19.322 \mathrm{~min}$ (minor); $86 \%$ ee.

(S)-1-(2,6-dichloro-3-fluorophenyl)ethan-1-ol (2m) ${ }^{8}$<smiles>C[C@H](O)c1c(Cl)ccc(F)c1Cl</smiles>

Colorless oil, $90 \%$ isolated yield, [a] $]_{\boldsymbol{D}}^{\mathbf{2 0}}=+3.5(\mathrm{c} 1.0 \mathrm{MeOH})\left[\mathrm{lit} .^{8}[\mathrm{a}]_{\boldsymbol{D}}^{\mathbf{2 0}}=+5.0(\mathrm{c} 1.35 \mathrm{in} \mathrm{MeOH}), 99 \%\right.$ ee, $\left.\mathrm{S}\right] .{ }^{1} \mathrm{H} \mathrm{NMR}(400 \mathrm{MHz}, \mathrm{CDCl} 3) \delta 7.35$ $-7.18(\mathrm{~m}, 1 \mathrm{H}), 7.02(\mathrm{t}, J=8.0 \mathrm{~Hz}, 1 \mathrm{H}), 5.57(\mathrm{~d}, J=6.4 \mathrm{~Hz}, 1 \mathrm{H}), 3.05(\mathrm{~s}, 1 \mathrm{H}), 1.64(\mathrm{~d}, J=6.4 \mathrm{~Hz}, 3 \mathrm{H}) .{ }^{13} \mathrm{C} \mathrm{NMR}(100 \mathrm{MHz}, \mathrm{CDCl}) \delta 157.26(\mathrm{~d}$, $J=247.6 \mathrm{~Hz}), 140.51(\mathrm{~s}), 129.60(\mathrm{~d}, J=7.5 \mathrm{~Hz}), 128.28(\mathrm{~d}, J=3.8 \mathrm{~Hz}), 121.38(\mathrm{~d}, J=18.8 \mathrm{~Hz}), 115.64(\mathrm{~d}, J=23.2 \mathrm{~Hz}), 68.33(\mathrm{~d}, J=2.2 \mathrm{~Hz})$, 21.25 (s). ${ }^{19} \mathrm{~F}$ NMR $\left(377 \mathrm{MHz}, \mathrm{CDCl}_{3}\right) \delta$-113.30. HPLC-separation conditions: Chiralcel OD-H, $254 \mathrm{~nm}$, hexane $:{ }^{i} \mathrm{PrOH}=120: 1$; flow rate 1.21 $\mathrm{mL} / \mathrm{min} ; \mathrm{t}_{1}=10.204 \min , \mathrm{t}_{2}=10.927 \min , \mathrm{t}_{\mathrm{R}}=9.874 \min$ (major), $\mathrm{t}_{\mathrm{R}}=10.514 \min ($ minor); $92 \%$ ee.

(R)-1-(2,6-dichlorophenyl)ethan-1-ol (2n) ${ }^{9}$<smiles>C[C@H](O)c1c(Cl)cccc1Cl</smiles>

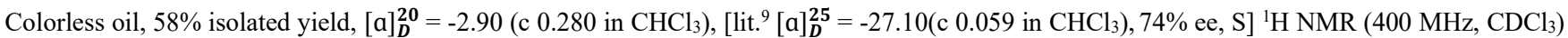
$\delta 7.32-7.23(\mathrm{~m}, 2 \mathrm{H}), 7.15-7.06(\mathrm{~m}, 1 \mathrm{H}), 5.59(\mathrm{q}, J=6.8 \mathrm{~Hz}, 1 \mathrm{H}), 2.97(\mathrm{~s}, 1 \mathrm{H}), 1.63(\mathrm{dd}, J=6.8,0.8 \mathrm{~Hz}, 3 \mathrm{H}) .{ }^{13} \mathrm{C} \mathrm{NMR}\left(100 \mathrm{MHz}, \mathrm{CDCl}_{3}\right) \delta$ 138.67, 133.87, 129.35, 128.67, 68.23, 21.49. HPLC-separation conditions: Chiralcel OD-H, $254 \mathrm{~nm}$, hexane $:{ }^{i} \operatorname{PrOH}=99: 1$; flow rate $=1.0$ $\mathrm{mL} / \mathrm{min} ; \mathrm{t}_{1}=11.583 \mathrm{~min}, \mathrm{t}_{2}=12.471 \mathrm{~min}, \mathrm{t}_{\mathrm{R}}=11.330 \min ($ major $), \mathrm{t}_{\mathrm{R}}=12.139 \min (\operatorname{minor}) ; 89 \%$ ee.

(S)-1-(2,6-dimethylphenyl)ethan-1-ol (2o) ${ }^{9}$<smiles>Cc1cccc(C)c1[C@H](C)O</smiles>

White solid , 45\% isolated yield, [a $]_{\boldsymbol{D}}^{\mathbf{2 0}}=-35.29$ (c 0.306 in $\left.\mathrm{CHCl}_{3}\right)$, [lit. ${ }^{9}[\mathrm{a}]_{\boldsymbol{D}}^{25}=-78.59$ (c 0.247 in $\left.\mathrm{CHCl}_{3}\right), 95 \%$ ee, S]. ${ }^{1} \mathrm{H} \mathrm{NMR}(400 \mathrm{MHz}$, $\left.\mathrm{CDCl}_{3}\right) \delta$ 7.08-6.99 (m, 3H), $5.39(\mathrm{q}, J=6.8 \mathrm{~Hz}, 1 \mathrm{H}), 2.46(\mathrm{~s}, 6 \mathrm{H}), 1.91-1.88(\mathrm{~m}, 1 \mathrm{H}), 1.54(\mathrm{~d}, J=6.8 \mathrm{~Hz}, 3 \mathrm{H}) .{ }^{13} \mathrm{C} \mathrm{NMR}\left(100 \mathrm{MHz}, \mathrm{CDCl}_{3}\right) \delta$ 140.60, 135.70, 129.42, 126.94, 67.65, 21.47, 20.64. HPLC-separation conditions: Chiralcel OD-H, $254 \mathrm{~nm}$, hexane $:{ }^{i} \operatorname{PrOH}=98: 2 ;$ flow rate $=$ $1.0 \mathrm{~mL} / \mathrm{min} ; \mathrm{t}_{1}=9.917 \mathrm{~min}, \mathrm{t}_{2}=12.372 \mathrm{~min}, \mathrm{t}_{\mathrm{R}}=9.717 \mathrm{~min}$ (major), $\mathrm{t}_{\mathrm{R}}=11685 \mathrm{~min}$ (minor). $89 \%$ ee. 
<smiles>CC(O)c1ccco1</smiles>

Colorless oil, 51\% isolated yield, [a] $]_{D}^{20}=-12.69$ (c 0.670 in $\left.\mathrm{CHCl}_{3}\right),\left[\mathrm{lit}^{5}{ }^{5}[\mathrm{a}]_{\boldsymbol{D}}^{25}=+12.87\right.$ (c 0.25 in $\left.\mathrm{CHCl}_{3}\right), 85 \%$ ee, R]. ${ }^{1} \mathrm{H} \mathrm{NMR}(400 \mathrm{MHz}$, $\left.\mathrm{CDCl}_{3}\right) \delta 7.37(\mathrm{dd}, J=1.7,0.6 \mathrm{~Hz}, 1 \mathrm{H}), 6.32(\mathrm{dd}, J=3.2,1.8 \mathrm{~Hz}, 1 \mathrm{H}), 6.22(\mathrm{~d}, J=3.2 \mathrm{~Hz}, 1 \mathrm{H}), 4.88(\mathrm{q}, J=6.6 \mathrm{~Hz}, 1 \mathrm{H}), 2.00(\mathrm{~s}, 1 \mathrm{H}), 1.54(\mathrm{~d}, J=$ $6.6 \mathrm{~Hz}, 3 \mathrm{H}) .{ }^{13} \mathrm{C}$ NMR $\left(100 \mathrm{MHz}, \mathrm{CDCl}_{3}\right) \delta 157.60,141.93,110.14,105.12,63.64,21.27$. The optical purity was determined by Agilent $6820 \mathrm{GC}$ with a CP-Chirasil-Dex CB column using a flame ionization detector (FID) operating at $280{ }^{\circ} \mathrm{C}$. Injector temperature was set at $250{ }^{\circ} \mathrm{C}$. $\left(60{ }^{\circ} \mathrm{C}\right.$ isothermal for 2 minutes, $2{ }^{\circ} \mathrm{C} / \mathrm{min}, 110{ }^{\circ} \mathrm{C}, 10{ }^{\circ} \mathrm{C} / \mathrm{min}, 170{ }^{\circ} \mathrm{C}$ for 5 minutes, $\mathrm{t}_{\mathrm{R}}=16.122 \mathrm{~min}$ and $16.707 \mathrm{~min}$ ). $t_{\mathrm{R}}=16.394$ min for minor isomer and $t_{\mathrm{R}}=16.697 \mathrm{~min}$ for major isomer. $68 \%$ ee.

(S)-1-(thiophen-2-yl)ethan-1-ol (2q) ${ }^{5}$<smiles>C[C@@H](O)c1cccs1</smiles>

Colorless oil, $53 \%$ isolated yield, $[\mathrm{a}]_{\boldsymbol{D}}^{\mathbf{2 0}}=-40.43$ (c 0.460 in $\left.\mathrm{CHCl}_{3}\right),\left[\right.$ lit. ${ }^{5}[\mathrm{a}]_{\boldsymbol{D}}^{25}=+23.70$ (c 1.0 in $\left.\mathrm{CHCl}_{3}\right), 90 \%$ ee, $\left.\mathrm{R}\right] .{ }^{1} \mathrm{H} \mathrm{NMR}\left(400 \mathrm{MHz}, \mathrm{CDCl}_{3}\right)$ $\delta 7.24(\mathrm{dd}, J=4.8,1.6 \mathrm{~Hz}, 1 \mathrm{H}), 7.01-6.92(\mathrm{~m}, 2 \mathrm{H}), 5.12(\mathrm{q}, J=6.4 \mathrm{~Hz}, 1 \mathrm{H}), 2.24(\mathrm{~s}, 1 \mathrm{H}), 1.59(\mathrm{~d}, J=6.4 \mathrm{~Hz}, 3 \mathrm{H}) .{ }^{13} \mathrm{C} \mathrm{NMR}(100 \mathrm{MHz}, \mathrm{CDCl} 3)$ $\delta 149.89,126.69,124.47,123.23,66.25,25.27$. The optical purity was determined by Agilent $6820 \mathrm{GC}$ with a CP-Chirasil-Dex CB column using a flame ionization detector (FID) operating at $280{ }^{\circ} \mathrm{C}$. Injector temperature was set at $250{ }^{\circ} \mathrm{C}$. $\left(80{ }^{\circ} \mathrm{C}\right.$ isothermal for 2 minutes, $2{ }^{\circ} \mathrm{C} / \mathrm{min}, 170{ }^{\circ} \mathrm{C}$ for 5 minutes, $t_{\mathrm{R}}=18.284 \mathrm{~min}$ and $\left.19.379 \mathrm{~min}\right) . t_{\mathrm{R}}=18.979 \mathrm{~min}$ for minor isomer and $t_{\mathrm{R}}=19.675 \mathrm{~min}$ for major isomer. $71 \%$ ee.

(S)-2,2-dimethyl-1-phenylpropan-1-ol (4a) ${ }^{10}$<smiles>CC(C)(C)[C@H](O)c1ccccc1</smiles>

White solid, $89 \%$ isolated yield, [a $]_{\boldsymbol{D}}^{20}=-34.50\left(\mathrm{c} 1.000\right.$ in $\left.\mathrm{CHCl}_{3}\right),\left[\mathrm{lit}^{10}[\mathrm{a}]_{\boldsymbol{D}}^{25}=+30.8\right.$ (c 0.40 in $\left.\mathrm{CHCl}_{3}\right), 85 \%$ ee, $\left.\mathrm{R}\right] .{ }^{1} \mathrm{H} \mathrm{NMR}(400 \mathrm{MHz}, \mathrm{CDCl} 3)$ $\delta 7.34-7.23(\mathrm{~m}, 5 \mathrm{H}), 4.40(\mathrm{~s}, 1 \mathrm{H}), 1.79(\mathrm{~s}, 1 \mathrm{H}), 0.92(\mathrm{~s}, 9 \mathrm{H}) .{ }^{13} \mathrm{C}$ NMR $\left(100 \mathrm{MHz}, \mathrm{CDCl}_{3}\right) \delta$ 142.22, 127.61, 127.56, 127.28, 82.43, 35.64, 25.93. HPLC-separation conditions: Chiralcel OD-H, $254 \mathrm{~nm}$, hexane $: i-\mathrm{PrOH}=99.5: 0.5$; flow rate $=1.0 \mathrm{~mL} / \mathrm{min} ; \mathrm{t}_{1}=6.521 \mathrm{~min}, \mathrm{t}_{2}=7.490 \mathrm{~min}, \mathrm{t}_{\mathrm{R}}=$ $6.504 \min$ (major), $\mathrm{t}_{\mathrm{R}}=7.640 \mathrm{~min}$ (minor). $91 \%$ ee.

(S)-2,2-dimethyl-1-(o-tolyl)propan-1-ol (4b) ${ }^{11}$<smiles>Cc1ccccc1[C@@H](O)C(C)(C)C</smiles>

Colorless oil, $94 \%$ isolated yield, [a] $]_{\boldsymbol{D}}^{\mathbf{2 0}}=-40.00\left(\mathrm{c} 0.250\right.$ in $\left.\mathrm{CHCl}_{3}\right),{ }^{1} \mathrm{H}$ NMR $\left(400 \mathrm{MHz}, \mathrm{CDCl}_{3}\right) \delta 7.49(\mathrm{dd}, J=8.0,1.6 \mathrm{~Hz}, 1 \mathrm{H}), 7.25-7.11(\mathrm{~m}$, $3 \mathrm{H}), 4.77(\mathrm{~s}, 1 \mathrm{H}), 2.37(\mathrm{~s}, 3 \mathrm{H}), 1.91-1.82(\mathrm{~m}, 1 \mathrm{H}), 0.99(\mathrm{~s}, 9 \mathrm{H}) .{ }^{13} \mathrm{C} \mathrm{NMR}\left(100 \mathrm{MHz}, \mathrm{CDCl}_{3}\right) \delta 140.88,135.75,130.19,127.59,127.01,125.48$, 76.73, 36.95, 26.09, 20.40. HPLC-separation conditions: Chiralcel OD-H, $254 \mathrm{~nm}$, hexane $: i$-PrOH $=99.5: 0.5 ;$ flow rate $=1.0 \mathrm{~mL} / \mathrm{min} ; \mathrm{t}_{1}=$ $16.397 \mathrm{~min}, \mathrm{t}_{2}=18.005 \mathrm{~min}, \mathrm{t}_{\mathrm{R}}=16.146 \mathrm{~min}$ (minor), $\mathrm{t}_{\mathrm{R}}=17.351 \mathrm{~min}$ (major). $92 \%$ ee.

(S)-1-(4-methoxyphenyl)-2,2-dimethylpropan-1-ol (4c) ${ }^{10}$<smiles>COc1ccc([C@@H](O)C(C)(C)C)cc1</smiles>

Colorless oil, $78 \%$ isolated yield, $[\mathrm{a}]_{\boldsymbol{D}}^{20}=-33.99\left(\mathrm{c}=0.712\right.$ in $\left.\mathrm{CHCl}_{3}\right),\left[\mathrm{lit} .{ }^{10}[\mathrm{a}]_{\boldsymbol{D}}^{24}=-35.5\left(\mathrm{c} 0.48\right.\right.$ in $\left.\mathrm{CHCl}_{3}\right), 96 \%$ ee, R]. ${ }^{1} \mathrm{H} \mathrm{NMR}(400 \mathrm{MHz}$, $\left.\mathrm{CDCl}_{3}\right) \delta 7.21(\mathrm{~d}, J=8.7 \mathrm{~Hz}, 2 \mathrm{H}), 6.84(\mathrm{~d}, J=8.7 \mathrm{~Hz}, 2 \mathrm{H}), 4.32(\mathrm{~s}, 1 \mathrm{H}), 3.79(\mathrm{~s}, 3 \mathrm{H}), 2.03(\mathrm{~s}, 1 \mathrm{H}), 0.90(\mathrm{~s}, 9 \mathrm{H}) .{ }^{13} \mathrm{C} \mathrm{NMR}\left(100 \mathrm{MHz}, \mathrm{CDCl}_{3}\right) \delta$ 
158.78, 134.49, 128.64, 112.94, 81.96, 55.22, 35.68, 25.93. HPLC-separation conditions: Chiralcel AD-H, $254 \mathrm{~nm}$, hexane $: i$-PrOH $=98: 2 ;$ flow rate $=1.0 \mathrm{~mL} / \mathrm{min} ; \mathrm{t}_{1}=16.725 \mathrm{~min}, \mathrm{t}_{2}=18.509 \mathrm{~min}, \mathrm{t}_{\mathrm{R}}=10.418 \mathrm{~min}($ minor $), \mathrm{t}_{\mathrm{R}}=11.858 \mathrm{~min}$ (major). $92 \%$ ee.

(S)-1-(2-bromophenyl)-2,2-dimethylpropan-1-ol (4d) ${ }^{12}$<smiles>CC(C)(C)[C@@H](O)c1ccccc1Br</smiles>

White solid, 76\% isolated yield, [a] $]_{D}^{20}=-25.90\left(\mathrm{c} 0.888 \mathrm{in} \mathrm{CHCl}_{3}\right),{ }^{1} \mathrm{H} \mathrm{NMR}\left(400 \mathrm{MHz}, \mathrm{CDCl}_{3}\right) \delta 7.52(\mathrm{t}, J=6.0 \mathrm{~Hz}, 2 \mathrm{H}), 7.30(\mathrm{t}, J=7.6 \mathrm{~Hz}, 1 \mathrm{H})$, $7.11(\mathrm{t}, J=7.2 \mathrm{~Hz}, 1 \mathrm{H}), 4.99(\mathrm{~s}, 1 \mathrm{H}), 2.05(\mathrm{~s}, 1 \mathrm{H}), 0.99(\mathrm{~s}, 9 \mathrm{H}) .{ }^{13} \mathrm{C} \mathrm{NMR}\left(100 \mathrm{MHz}, \mathrm{CDCl}_{3}\right) \delta 141.54,132.57,129.66,128.72,126.91,124.10$, 78.95, 37.03, 25.94. HPLC-separation conditions: Chiralcel OD-H, $254 \mathrm{~nm}$, hexane $: i-\mathrm{PrOH}=99.5: 0.5$; flow rate $=1.0 \mathrm{~mL} / \mathrm{min} ; \mathrm{t}_{1}=15.486 \mathrm{~min}$, $\mathrm{t}_{2}=17.395 \min , \mathrm{t}_{\mathrm{R}}=15.625 \min$ (minor), $\mathrm{t}_{\mathrm{R}}=17.060 \min$ (major). $93 \%$ ee.

(S)-1-(3-bromophenyl)-2,2-dimethylpropan-1-ol (4e)<smiles>CC(C)(C)[C@@H](O)c1cccc(Br)c1</smiles>

Colorless oil, $81 \%$ isolated yield, [a] $]_{\boldsymbol{D}}^{\mathbf{2 0}}=-18.77\left(\mathrm{c} 0.810\right.$ in $\left.\mathrm{CHCl}_{3}\right),{ }^{1} \mathrm{H} \mathrm{NMR}\left(400 \mathrm{MHz}, \mathrm{CDCl}_{3}\right) \delta 7.45(\mathrm{~d}, J=1.6 \mathrm{~Hz}, 1 \mathrm{H}), 7.38(\mathrm{dt}, J=7.6,1.6$ $\mathrm{Hz}, 1 \mathrm{H}), 7.23-7.13(\mathrm{~m}, 2 \mathrm{H}), 4.30(\mathrm{~s}, 1 \mathrm{H}), 2.13(\mathrm{~s}, 1 \mathrm{H}), 0.90(\mathrm{~s}, 9 \mathrm{H}) .{ }^{13} \mathrm{C} \mathrm{NMR}\left(100 \mathrm{MHz}, \mathrm{CDCl}_{3}\right) \delta 144.54,130.61,130.34,129.12,126.35$, 121.84, 81.69, 35.66, 25.86. HRMS (ESI-MS) $[\mathrm{M}+\mathrm{Na}]^{+}$: calculated for $\left[\mathrm{C}_{11} \mathrm{H}_{15} \mathrm{BrNaO}\right]^{+}: 265.0198$, found: 265.0197. HPLC-separation conditions: Chiralcel OD-H, $254 \mathrm{~nm}$, hexane $: i-\mathrm{PrOH}=98: 2$; flow rate $=1.0 \mathrm{~mL} / \mathrm{min} ; \mathrm{t}_{1}=9.477 \mathrm{~min}, \mathrm{t}_{2}=14.486 \mathrm{~min}, \mathrm{t}_{\mathrm{R}}=9.730$ min $($ major$), \mathrm{t}_{\mathrm{R}}=16.534$ $\min$ (minor). $89 \%$ ee.

(S)-1-(4-bromophenyl)-2,2-dimethylpropan-1-ol (4f) ${ }^{13}$<smiles>CC(C)(C)[C@H](O)c1ccc(Br)cc1</smiles>

White solid, 87\% isolated yield, [a] $]_{D}^{20}=-24.30\left(\mathrm{c} 1.00\right.$ in $\left.\mathrm{CHCl}_{3}\right),{ }^{1} \mathrm{H} \mathrm{NMR}\left(400 \mathrm{MHz}, \mathrm{CDCl}_{3}\right) \delta 7.47(\mathrm{~d}, J=8.0 \mathrm{~Hz}, 2 \mathrm{H}), 7.20(\mathrm{~d}, J=8.0 \mathrm{~Hz}, 2 \mathrm{H})$, $4.36(\mathrm{~s}, 1 \mathrm{H}), 2.10(\mathrm{~s}, 1 \mathrm{H}), 0.94(\mathrm{~s}, 9 \mathrm{H}) .{ }^{13} \mathrm{C}$ NMR $\left(100 \mathrm{MHz}, \mathrm{CDCl}_{3}\right) \delta 141.09,130.62,129.32,121.09,81.71,35.56$, 25.81. HPLC-separation conditions: Chiralcel OD-H, $254 \mathrm{~nm}$, hexane : $i-\mathrm{PrOH}=98: 2$; flow rate $=1.0 \mathrm{~mL} / \mathrm{min} ; \mathrm{t}_{1}=11.220 \mathrm{~min}, \mathrm{t}_{2}=12.362 \mathrm{~min}, \mathrm{t}_{\mathrm{R}}=10.418 \mathrm{~min}(\mathrm{major})$, $\mathrm{t}_{\mathrm{R}}=11.858 \mathrm{~min}$ (minor). $93 \%$ ee.

(S)-1-phenylpropan-1-ol (4g) ${ }^{5}$<smiles>CC[C@H](O)c1ccccc1</smiles>

Colorless oil, $82 \%$ isolated yield, [a $]_{D}^{20}=-35.92\left(\mathrm{c} 0.284\right.$ in $\left.\mathrm{CHCl}_{3}\right),\left[\mathrm{lit}^{5}{ }^{5}[\mathrm{a}]_{\boldsymbol{D}}^{25}=+38.07\left(\mathrm{c}=0.5\right.\right.$ in $\left.\mathrm{CHCl}_{3}\right), 89 \%$ ee, $\left.\mathrm{R}\right] .{ }^{1} \mathrm{H} \mathrm{NMR}(400 \mathrm{MHz}$, $\left.\mathrm{CDCl}_{3}\right) \delta 7.45-7.14(\mathrm{~m}, 5 \mathrm{H}), 4.54(\mathrm{t}, J=8.0 \mathrm{~Hz}, 1 \mathrm{H}), 2.12(\mathrm{~s}, 1 \mathrm{H}), 1.76(\mathrm{~m}, 2 \mathrm{H}), 0.89(\mathrm{t}, J=8.0 \mathrm{~Hz}, 3 \mathrm{H}) .{ }^{13} \mathrm{C} \mathrm{NMR}\left(100 \mathrm{MHz}, \mathrm{CDCl}_{3}\right) \delta 144.62$, 128.39, 127.47, 126.01, 75.99, 31.87, 10.15.HPLC-separation conditions: Chiralcel OD-H, $254 \mathrm{~nm}$, hexane: ${ }^{\mathrm{PrOH}}=0.980 \mathrm{~mL} / \mathrm{min}: 0.020 \mathrm{~mL} / \mathrm{min}$; $\mathrm{t}_{1}=11.855 \mathrm{~min}, \mathrm{t}_{2}=15.461 \mathrm{~min}, \mathrm{t}_{\mathrm{R}}=12.341 \min \left(\right.$ minor), $\mathrm{t}_{\mathrm{R}}=15.149 \min$ (major). $79 \%$ ee.

(S)-2-methyl-1-phenylpropan-1-ol (4h) ${ }^{5}$<smiles>CC(C)[C@H](O)c1ccccc1</smiles>

Colorless oil, $82 \%$ isolated yield, [a] $]_{D}^{20}-32.74$ (c 0.446 in $\left.\mathrm{CHCl}_{3}\right)$, [lit. ${ }^{5}[\mathrm{a}]_{D}^{25}=+25.60\left(\mathrm{c} 0.25\right.$ in $\left.\mathrm{CHCl}_{3}\right), 92 \%$ ee, R]. ${ }^{1} \mathrm{H} \mathrm{NMR}\left(400 \mathrm{MHz}, \mathrm{CDCl}_{3}\right)$ $\delta$ 7.37-7.21 (m, 5H), $4.32(\mathrm{~d}, J=7.2 \mathrm{~Hz}, 1 \mathrm{H}), 2.04(\mathrm{~s}, 1 \mathrm{H}), 2.00-1.86(\mathrm{~m}, 1 \mathrm{H}), 0.98(\mathrm{~d}, J=6.8 \mathrm{~Hz}, 3 \mathrm{H}), 0.78(\mathrm{~d}, J=6.8 \mathrm{~Hz}, 3 \mathrm{H}) .{ }^{13} \mathrm{C} \mathrm{NMR}(100$ 
$\left.\mathrm{MHz}, \mathrm{CDCl}_{3}\right) \delta 143.70,128.17,127.39,126.60,80.02,35.26,19.00,18.25$. HPLC-separation conditions: Chiralcel OD-H, $254 \mathrm{~nm}$, hexane $: i$ $\operatorname{PrOH}=100: 1 ;$ flow rate $=1.01 \mathrm{~mL} / \mathrm{min} ; \mathrm{t}_{1}=16.480 \mathrm{~min}, \mathrm{t}_{2}=18.146 \mathrm{~min}, \mathrm{t}_{\mathrm{R}}=16.914 \mathrm{~min}$ (minor), $\mathrm{t}_{\mathrm{R}}=17.966 \mathrm{~min}$ (major). $82 \%$ ee.

(S)-1-phenylbutan-1-ol (4i) ${ }^{5}$<smiles>CCC[C@@H](O)c1ccccc1</smiles>

White solid, 79\% isolated yield, [a $]_{\boldsymbol{D}}^{\mathbf{2 0}}=-36.11$ (c 0.648 in $\left.\mathrm{CHCl}_{3}\right)$, [lit. ${ }^{5}[\mathrm{a}]_{\boldsymbol{D}}^{\mathbf{2 5}}=+52.57$ (c 1.0 in $\left.\mathrm{CHCl}_{3}\right), 89 \%$ ee, R]. ${ }^{1} \mathrm{H} \mathrm{NMR}\left(400 \mathrm{MHz}, \mathrm{CDCl}_{3}\right)$ $\delta 7.38(\mathrm{~s}, 4 \mathrm{H}), 7.32(\mathrm{~s}, 1 \mathrm{H}), 4.70(\mathrm{t}, J=6.0 \mathrm{~Hz}, 1 \mathrm{H}), 2.20(\mathrm{~s}, 1 \mathrm{H}), 1.82(\mathrm{~s}, 1 \mathrm{H}), 1.75-1.73(\mathrm{~m}, 1 \mathrm{H}), 1.55-1.42(\mathrm{~m}, 1 \mathrm{H}), 1.40-1.34(\mathrm{~m}, 1 \mathrm{H}), 0.98$ $(\mathrm{t}, J=7.2 \mathrm{~Hz}, 3 \mathrm{H}) .{ }^{13} \mathrm{C}$ NMR $\left(100 \mathrm{MHz}, \mathrm{CDCl}_{3}\right) \delta 144.97,128.40,127.44,125.93,74.38,41.23,19.04,13.98$. HPLC-separation conditions: Chiralcel OD-H, $254 \mathrm{~nm}$, hexane $: i-\mathrm{PrOH}=98: 2$; flow rate $=1.0 \mathrm{~mL} / \mathrm{min} ; \mathrm{t}_{1}=12.251 \mathrm{~min}, \mathrm{t}_{2}=14.101 \mathrm{~min}, \mathrm{t}_{\mathrm{R}}=12.309$ min $(\operatorname{minor}), \mathrm{t}_{\mathrm{R}}=14.095$ $\min .69 \%$ ee.

(S)-cyclopentyl(phenyl)methanol (4j) ${ }^{14}$<smiles>O[C@H](c1ccccc1)C1CCCC1</smiles>

Colorless oil, $82 \%$ isolated yield, [a] ${ }_{D}^{20}=-36.08\left(\mathrm{c}=0.352\right.$ in $\left.\mathrm{CHCl}_{3}\right),\left[\right.$ lit. ${ }^{14}=-42.1$ (c 0.70 in $\left.\mathrm{CHCl}_{3}\right),>99 \%$ ee, $\left.\mathrm{S}\right] .{ }^{1} \mathrm{H} \mathrm{NMR}\left(400 \mathrm{MHz}, \mathrm{CDCl}_{3}\right) \delta$ $7.39(\mathrm{~s}, 4 \mathrm{H}), 7.33(\mathrm{~d}, J=4.0 \mathrm{~Hz}, 1 \mathrm{H}), 4.43(\mathrm{~d}, J=8.4 \mathrm{~Hz}, 1 \mathrm{H}), 2.32-2.24(\mathrm{~m}, 1 \mathrm{H}), 2.21(\mathrm{~s}, 1 \mathrm{H}), 2.04-1.87(\mathrm{~m}, 1 \mathrm{H}), 1.81-1.49(\mathrm{~m}, 5 \mathrm{H}), 1.44-1.42$ $(\mathrm{m}, 1 \mathrm{H}), 1.26-1.17(\mathrm{~m}, 1 \mathrm{H}) .{ }^{13} \mathrm{C}$ NMR $\left(100 \mathrm{MHz}, \mathrm{CDCl}_{3}\right) \delta 144.52,128.31,127.49,126.54,79.09,47.66,29.54,29.45,25.55,25.43$. HPLCseparation conditions: Chiralcel OD-H, $254 \mathrm{~nm}$, hexane $: i-\mathrm{PrOH}=98: 2$; flow rate $=1.0 \mathrm{~mL} / \mathrm{min} ; \mathrm{t}_{1}=12.313 \mathrm{~min}, \mathrm{t}_{2}=13.716 \mathrm{~min}, \mathrm{t}_{\mathrm{R}}=12.202$ $\min$ (minor), $t_{\mathrm{R}}=13.804 \min$ (major). $73 \%$ ee.

(S)-cyclohexyl(phenyl)methanol (4k) ${ }^{14}$<smiles>O[C@H](c1ccccc1)C1CCCCC1</smiles>

White solid, $85 \%$ isolated yield, $[\mathrm{a}]_{\boldsymbol{D}}^{20}=-26.61\left(\mathrm{c}=0.872\right.$ in $\left.\mathrm{CHCl}_{3}\right),\left[\mathrm{lit}^{2}{ }^{14}[\mathrm{a}]_{\boldsymbol{D}}^{25}=+31.93\left(\mathrm{c}=1.00\right.\right.$ in $\left.\mathrm{CHCl}_{3}\right), 94 \%$ ee, R]. ${ }^{1} \mathrm{H} \mathrm{NMR}(400 \mathrm{MHz}$, $\left.\mathrm{CDCl}_{3}\right) \delta$ 7.48-7.24 (m, 5H), $4.39(\mathrm{~d}, J=6.8 \mathrm{~Hz}, 1 \mathrm{H}), 2.13(\mathrm{~s}, 1 \mathrm{H}), 2.04(\mathrm{~d}, J=12.0 \mathrm{~Hz}, 1 \mathrm{H}), 1.83(\mathrm{~d}, J=12.0 \mathrm{~Hz}, 1 \mathrm{H}), 1.71(\mathrm{~s}, 3 \mathrm{H}), 1.43(\mathrm{~d}, J=$ $13.2 \mathrm{~Hz}, 1 \mathrm{H}), 1.37-1.07(\mathrm{~m}, 4 \mathrm{H}), 1.05-0.92(\mathrm{~m}, 1 \mathrm{H}) .{ }^{13} \mathrm{C} \mathrm{NMR}\left(100 \mathrm{MHz}, \mathrm{CDCl}_{3}\right) \delta$ 143.67, 128.17, 127.38, 126.67, 79.35, 44.95, 29.31, 28.87, 26.46, 26.13, 26.05. HPLC-separation conditions: Chiralcel OD-H, $254 \mathrm{~nm}$, hexane $: i-\mathrm{PrOH}=120: 1$; flow rate $=1.210 \mathrm{~mL} / \mathrm{min} ; \mathrm{t}_{1}=16.159 \mathrm{~min}$, $\mathrm{t}_{2}=18.160 \mathrm{~min}, \mathrm{t}_{\mathrm{R}}=16.278 \min$ (minor), $\mathrm{t}_{\mathrm{R}}=18.695 \min$ (major). $71 \%$ ee.

(S)-1-(2,4-dichlorophenyl)propan-1-ol (4I) ${ }^{8}$<smiles>CC[C@H](O)c1ccc(Cl)cc1Cl</smiles>

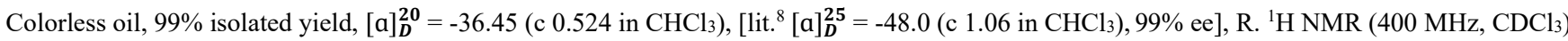
$\delta 7.42(\mathrm{~d}, J=8.4 \mathrm{~Hz}, 1 \mathrm{H}), 7.31(\mathrm{~d}, J=2.0 \mathrm{~Hz}, 1 \mathrm{H}), 7.26-7.22(\mathrm{~m}, 1 \mathrm{H}), 4.97(\mathrm{dd}, J=7.6,5.2 \mathrm{~Hz}, 1 \mathrm{H}), 2.56(\mathrm{~s}, 1 \mathrm{H}), 1.83-1.57(\mathrm{~m}, 2 \mathrm{H}), 0.94(\mathrm{t}, J$ $=7.2 \mathrm{~Hz}, 3 \mathrm{H}) .{ }^{13} \mathrm{C}$ NMR $\left(101 \mathrm{MHz}, \mathrm{CDCl}_{3}\right) \delta 140.63$, 133.30, 132.47, 129.02, 128.14, 127.31, 71.42, 30.47, 9.88. HPLC-separation conditions: Chiralcel OD-H, $254 \mathrm{~nm}$, hexane $: i-\mathrm{PrOH}=99: 1$; flow rate $=1.0 \mathrm{~mL} / \mathrm{min} ; \mathrm{t}_{1}=19.612 \mathrm{~min}, \mathrm{t}_{2}=24.688 \mathrm{~min}, \mathrm{t}_{\mathrm{R}}=20.155$ min $($ major $), \mathrm{t}_{\mathrm{R}}=24.222$ $\min$ (minor). $88 \%$ ee.

(S)-1-(naphthalen-1-yl)ethan-1-ol (4m) ${ }^{5}$<smiles>C[C@H](O)c1cccc2ccccc12</smiles> 
Colorless oil, $75 \%$ isolated yield, [a $]_{D}^{20}=-52.88\left(\mathrm{c} 0.312\right.$ in $\left.\mathrm{CHCl}_{3}\right),\left[\mathrm{lit}^{5}{ }^{5}[\mathrm{a}]_{D}^{25}=+58.63\right.$ (c 1.00 in $\left.\mathrm{CHCl}_{3}\right), 90 \%$ ee, R]. ${ }^{1} \mathrm{H} \mathrm{NMR}(400 \mathrm{MHz}$, $\left.\mathrm{CDCl}_{3}\right) \delta 8.10(\mathrm{~d}, J=8.8 \mathrm{~Hz}, 1 \mathrm{H}), 7.93-7.85(\mathrm{~m}, 1 \mathrm{H}), 7.79(\mathrm{~d}, J=8.4 \mathrm{~Hz}, 1 \mathrm{H}), 7.67(\mathrm{~d}, J=6.8 \mathrm{~Hz}, 1 \mathrm{H}), 7.58-7.44(\mathrm{~m}, 3 \mathrm{H}), 5.63(\mathrm{q}, J=6.4 \mathrm{~Hz}$, $1 \mathrm{H}), 2.32-2.15(\mathrm{~m}, 1 \mathrm{H}), 1.66(\mathrm{~d}, J=6.4 \mathrm{~Hz}, 3 \mathrm{H}) .{ }^{13} \mathrm{C} \mathrm{NMR}\left(100 \mathrm{MHz}, \mathrm{CDCl}_{3}\right) \delta 141.43,133.85,130.33,128.92,127.93,126.05,125.57,123.22$, 122.05, 67.09, 24.38. HPLC-separation conditions: Chiralcel OD-H, $220 \mathrm{~nm}$, hexane : $i-\mathrm{PrOH}=90: 10$; flow rate $=1.0 \mathrm{~mL} / \mathrm{min} ; \mathrm{t}_{1}=9.382 \mathrm{~min}, \mathrm{t}_{2}$ $=14.000 \mathrm{~min}, \mathrm{t}_{\mathrm{R}}=9.350 \mathrm{~min}$ (major), $\mathrm{t}_{\mathrm{R}}=13.995 \mathrm{~min}$ (minor). $87 \%$ ee.

(S)-1-(anthracen-9-yl)ethan-1-ol (4n) ${ }^{15}$<smiles>C[C@H](O)c1c2ccccc2cc2ccccc12</smiles>

White solid, 78\% isolated yield, [a $]_{D}^{20}=-27.40\left(\mathrm{c} 0.292\right.$ in $\left.\mathrm{CHCl}_{3}\right)$, [lit. ${ }^{15}[\mathrm{a}]_{D}^{25}=+18.5\left(\mathrm{c}=1.00\right.$ in $\left.\mathrm{CHCl}_{3}\right),>99 \%$ ee, R]. ${ }^{1} \mathrm{H} \mathrm{NMR}(400 \mathrm{MHz}$, $\left.\mathrm{CDCl}_{3}\right) \delta 8.44(\mathrm{~s}, 2 \mathrm{H}), 8.18(\mathrm{~s}, 1 \mathrm{H}), 7.92-7.78(\mathrm{~m}, 2 \mathrm{H}), 7.43-7.27(\mathrm{~m}, 4 \mathrm{H}), 6.08(\mathrm{q}, J=6.8 \mathrm{~Hz}, 1 \mathrm{H}), 2.62(\mathrm{~s}, 1 \mathrm{H}), 1.66(\mathrm{~d}, J=6.8 \mathrm{~Hz}, 3 \mathrm{H}) .{ }^{13} \mathrm{C}$ NMR $\left(101 \mathrm{MHz}, \mathrm{CDCl}_{3}\right) \delta 135.82,131.67,129.35,128.76,127.86,125.48,124.84,124.79,67.07,23.37$. HPLC-separation conditions: Chiralcel $\mathrm{OD}-\mathrm{H}, 254 \mathrm{~nm}$, hexane $: i-\mathrm{PrOH}=95: 5$; flow rate $=1.0 \mathrm{~mL} / \mathrm{min} ; \mathrm{t}_{1}=18.674 \mathrm{~min}, \mathrm{t}_{2}=31.203 \mathrm{~min}, \mathrm{t}_{\mathrm{R}}=18.676$ min $($ major$), \mathrm{t}_{\mathrm{R}}=31.304 \mathrm{~min}$ (minor). $92 \%$ ee. 


\section{Synthesis and characterization of Mn-H from complex Mn-1 ${ }^{16}$}

In a glove box, $20 \mathrm{mg}$ complex Mn-1 was dissolved in $1 \mathrm{~mL}$ of Toluene-d 8 containing $5.1 \mathrm{mg} \mathrm{KO}{ }^{t} \mathrm{Bu}(2 \mathrm{equiv}$.). $i$-PrOH (50 $\mu \mathrm{L}$, 14 equiv.) was added to this solution with vigorous stirring. Then the sample was immediately analyzed by ${ }^{1} \mathrm{H}$ NMR.
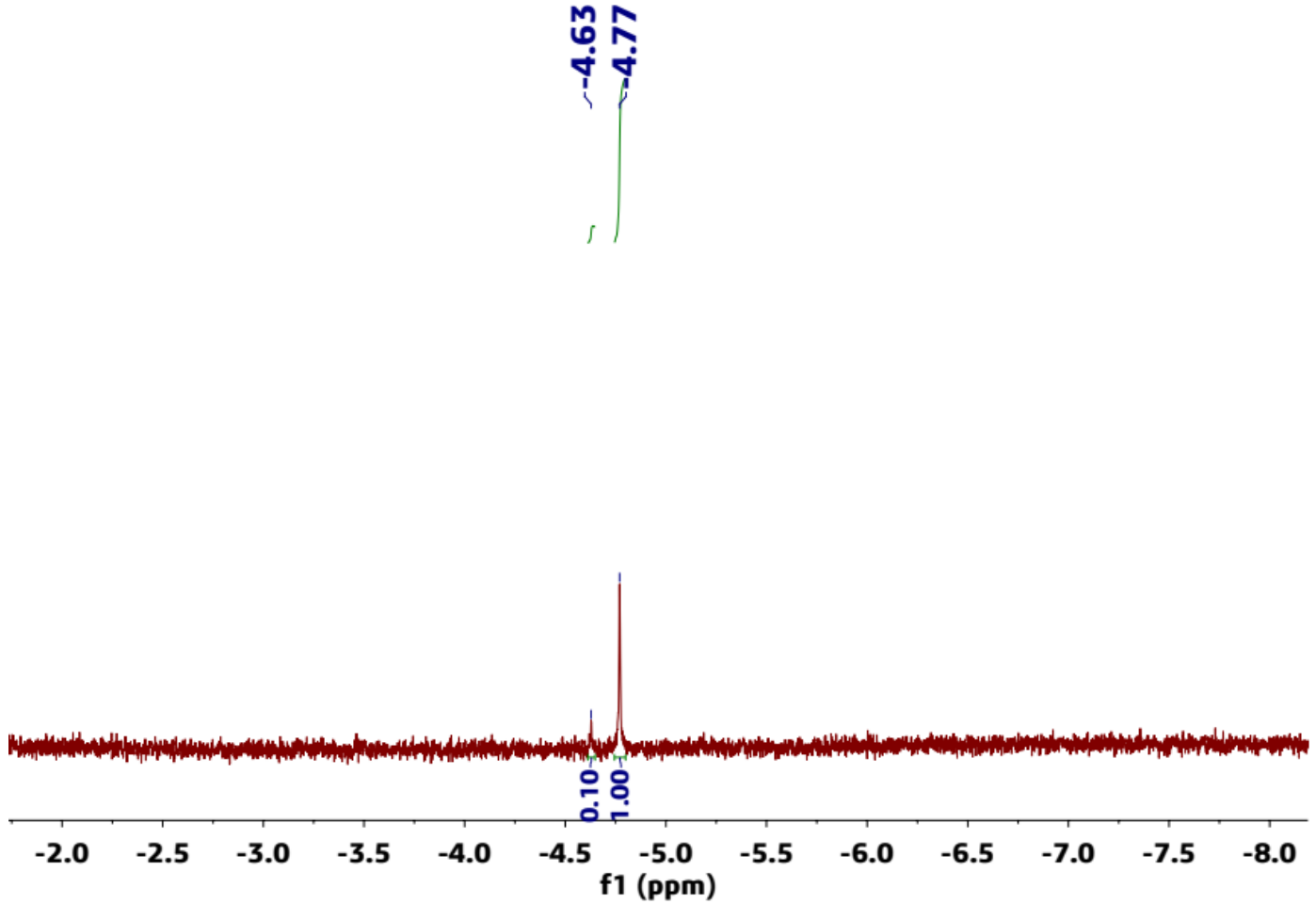
5. KIE experiment for the ATH of acetophenone with Mn-1 as catalyst (i-PrOH :deuterated i-PrOH $=$ $1: 1$, reaction at room temperature for $10 \mathrm{~min}, 13 \%$ yield of alcohols.)

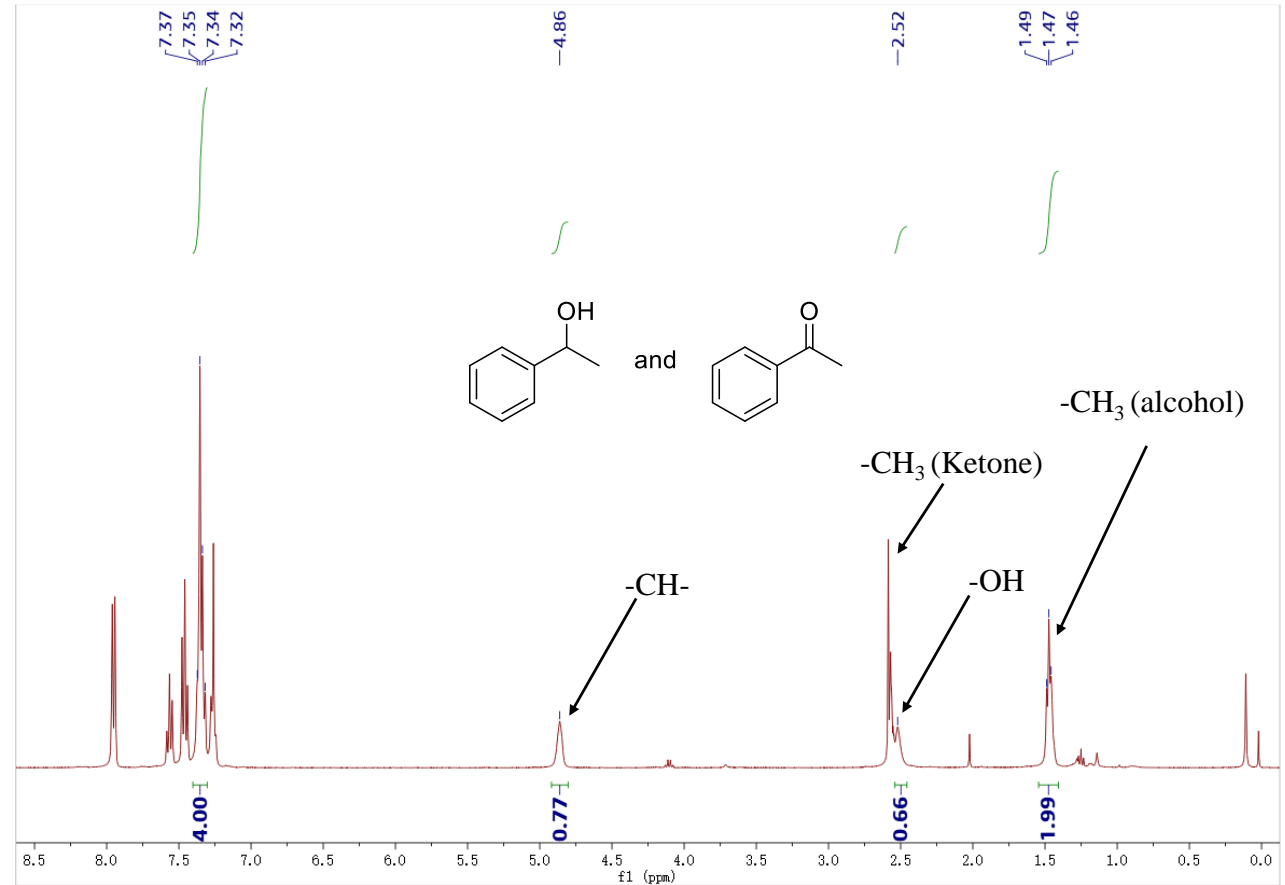

${ }^{1} \mathrm{H}$ NMR of mixture of KIE experiment after reaction of $10 \mathrm{~min}$ (the ratio of $\mathrm{H}$ at $7.35 \mathrm{ppm}$ and at $4.86 \mathrm{ppm}$ is $4: 0.77$. It indicates that $23 \%$ of $\alpha$-C$\mathrm{H}$ is deuterated (KIE value of 3.3.).

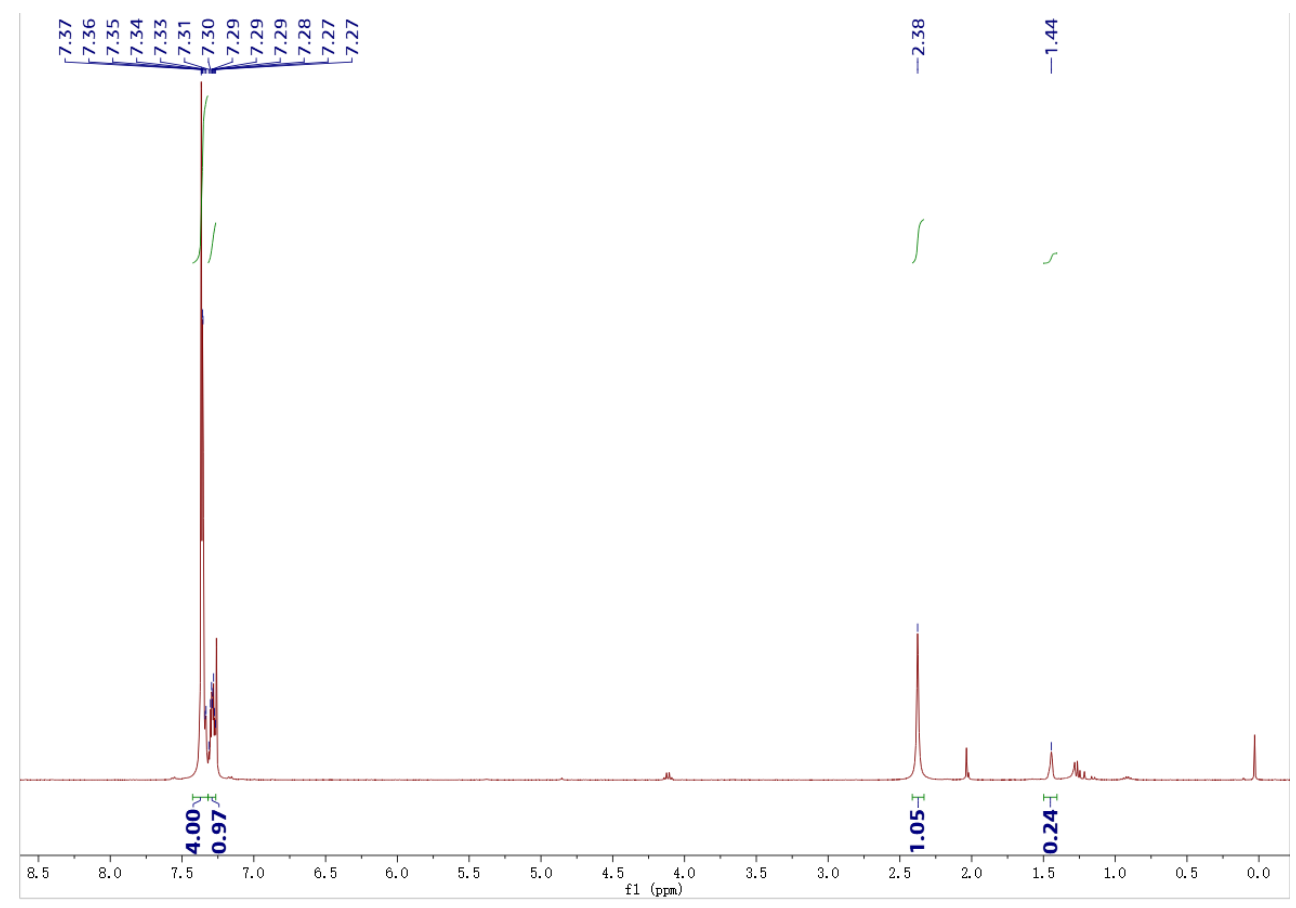

${ }^{1} \mathrm{H}$ NMR of $\alpha$-methylbenzyl alcohol with deuterated i-propanol under optimized conditions, no $\alpha$-C-H was observed. 


\section{Copies of NMR spectra}

(S)-2,2-dimethyl-1-(1-methyl-1H-benzo[d]imidazol-2-yl)propan-1-amine (L1)

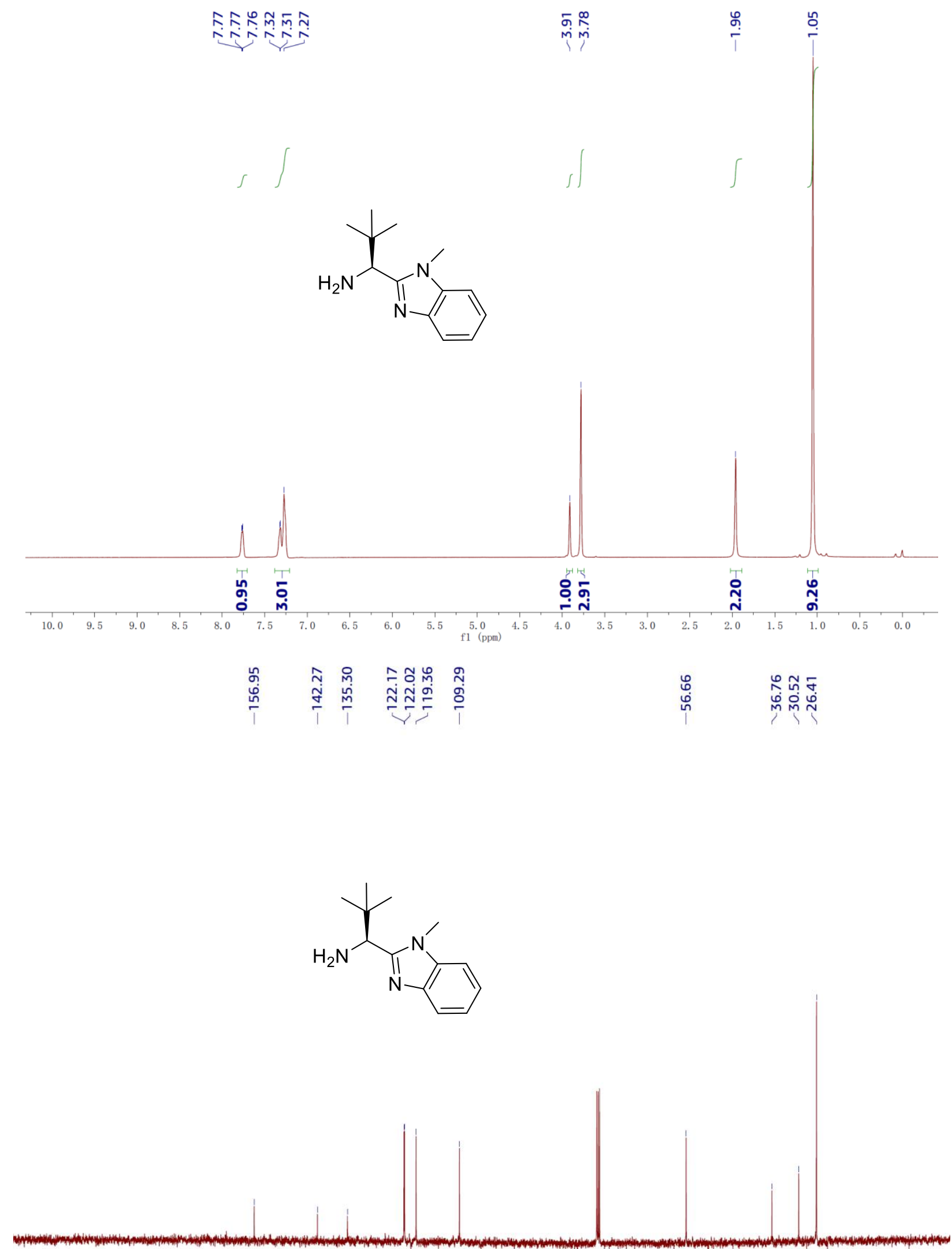

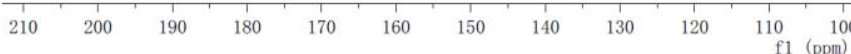


(S)-1-(1-ethyl-1H-benzo[d]imidazol-2-yl)-2,2-dimethylpropan-1-amine (L2)
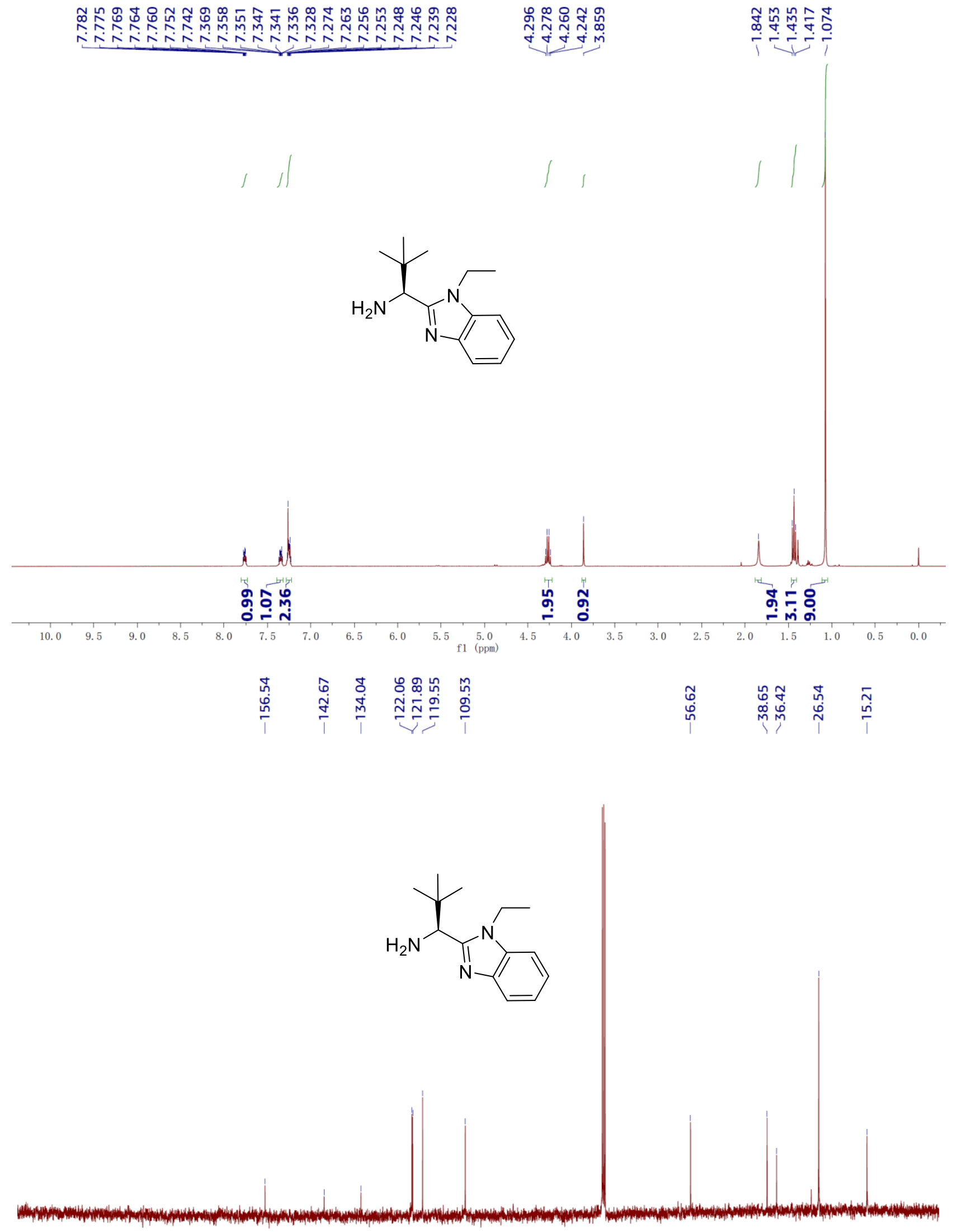

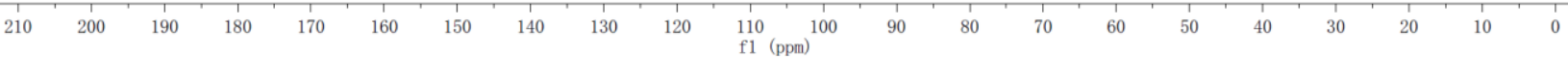


(S)-2-methyl-1-(1-methyl-1H-benzo[d]imidazol-2-yl)propan-1-amine (L3)
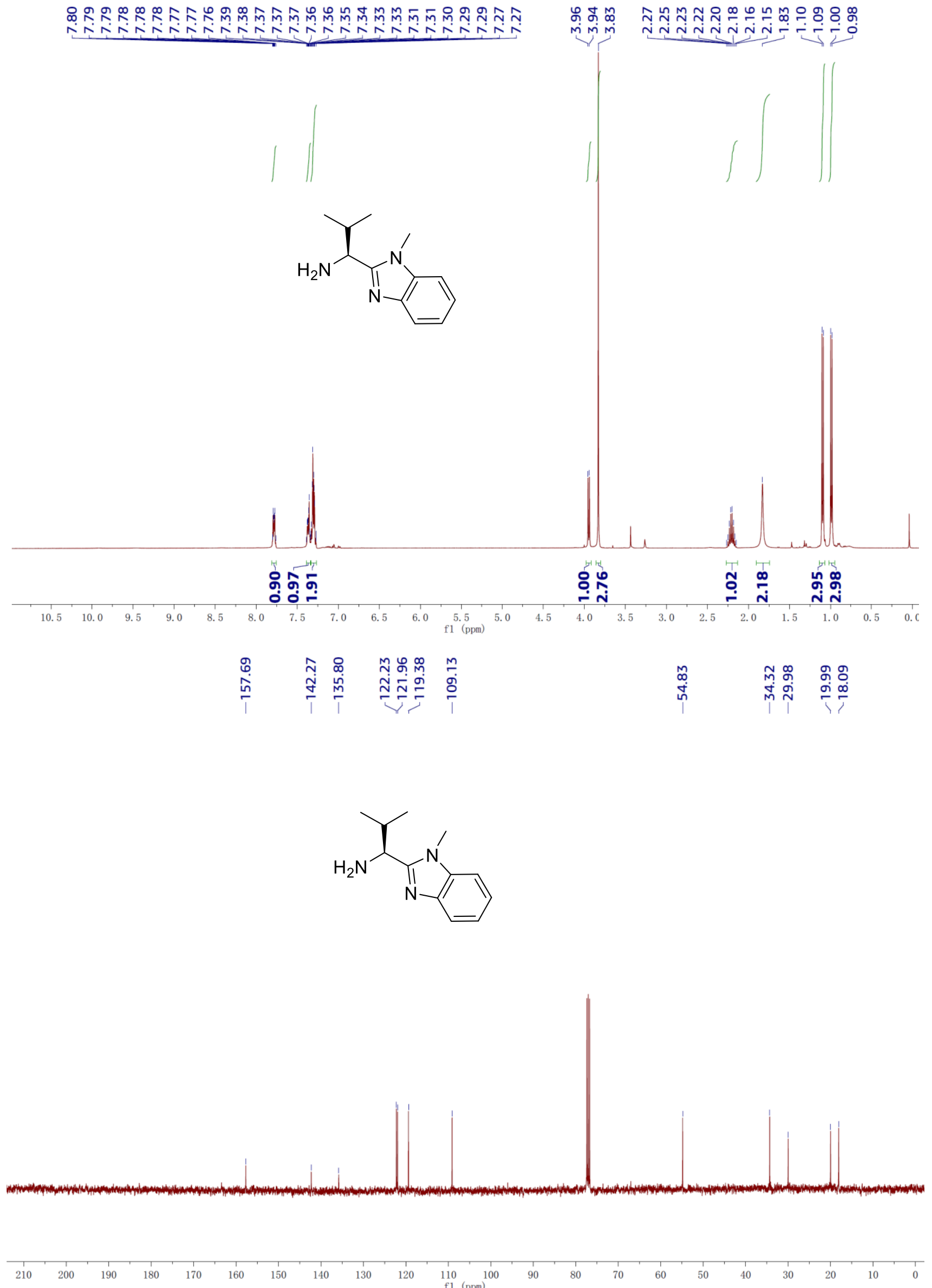
(1S)-((1r,3S)-adamantan-1-yl)(1-methyl-1H-benzo[d]imidazol-2-yl)methanamin (L4)

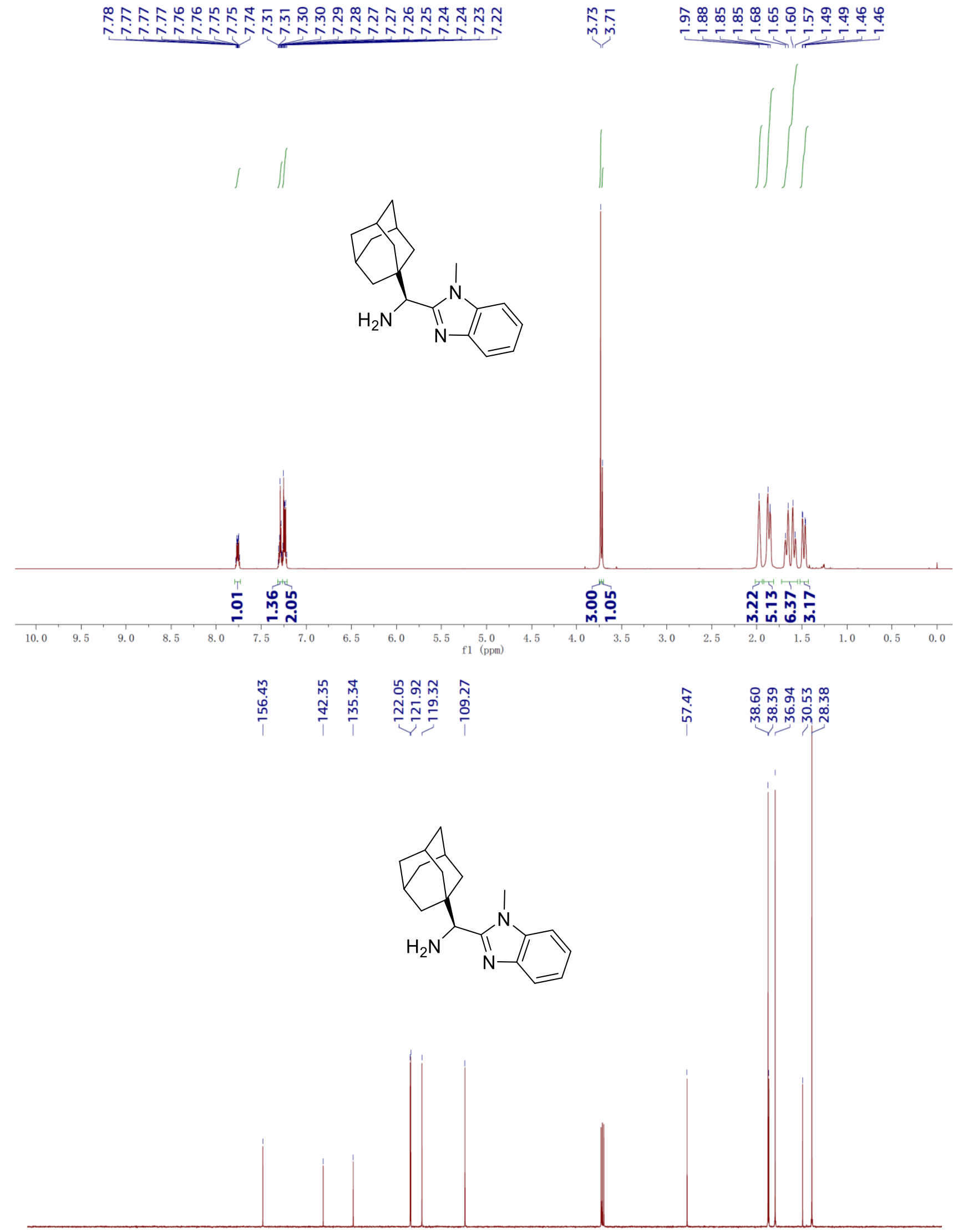

M下 $\quad$ ติ 
(S)-1-methyl-2-(pyrrolidin-2-yl)-1H-benzo[d]imidazole (L5)

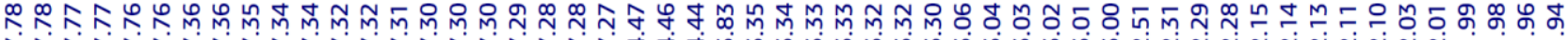

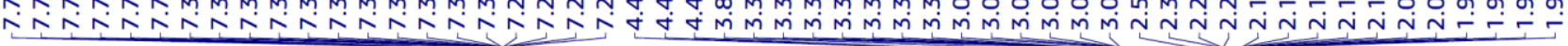

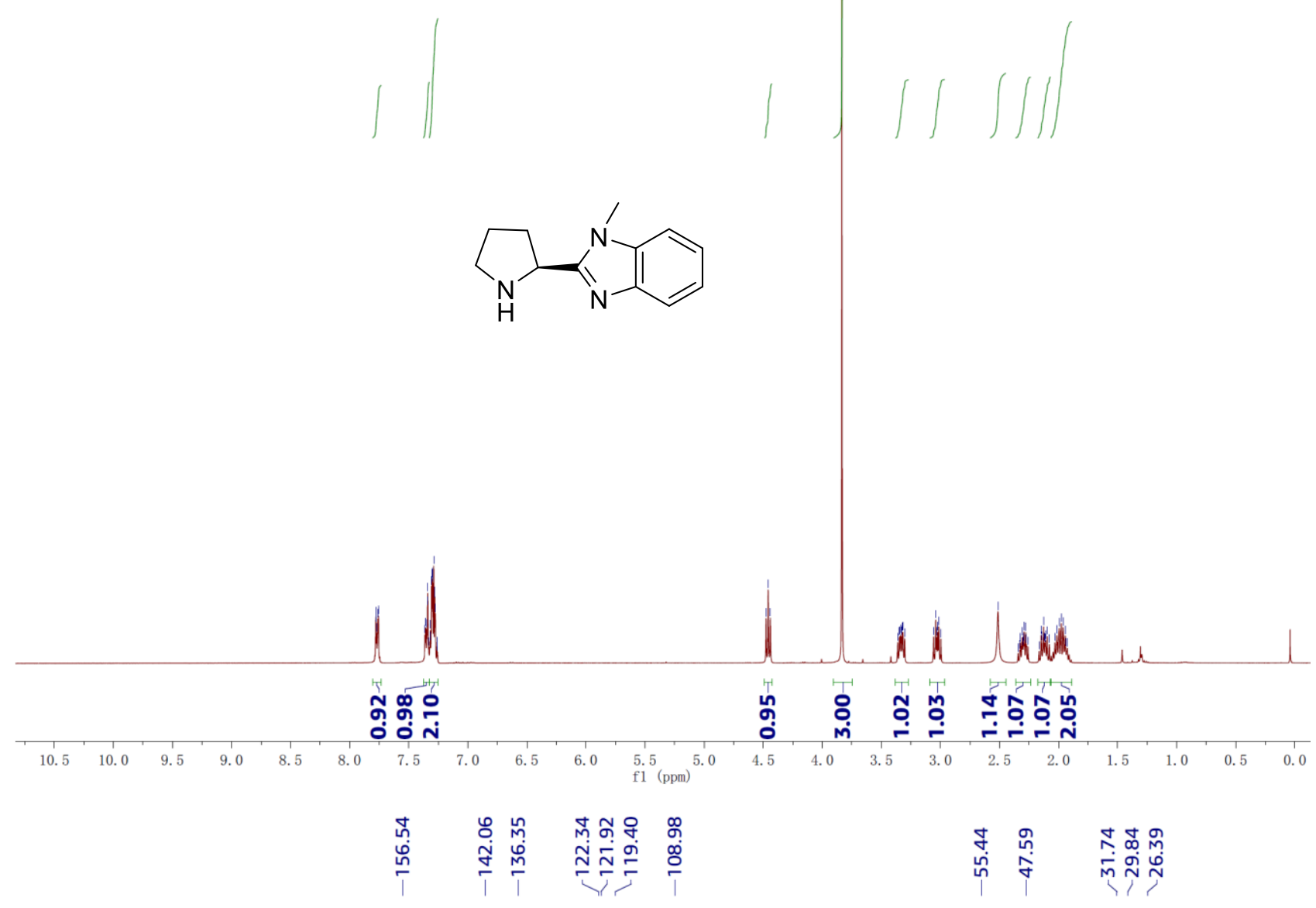<smiles>Cn1c(C2CCCN2)nc2ccccc21</smiles>

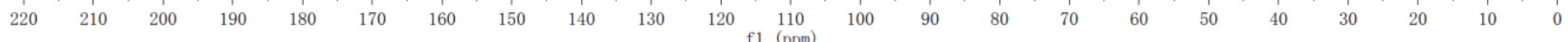


tert-butyl (S)-2-(4-bromo-1-methyl-1H-benzo[d]imidazol-2-yl)pyrrolidine-1-carboxylate(A4)

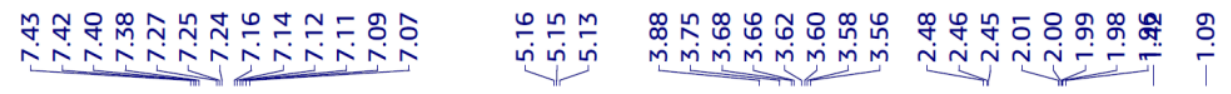
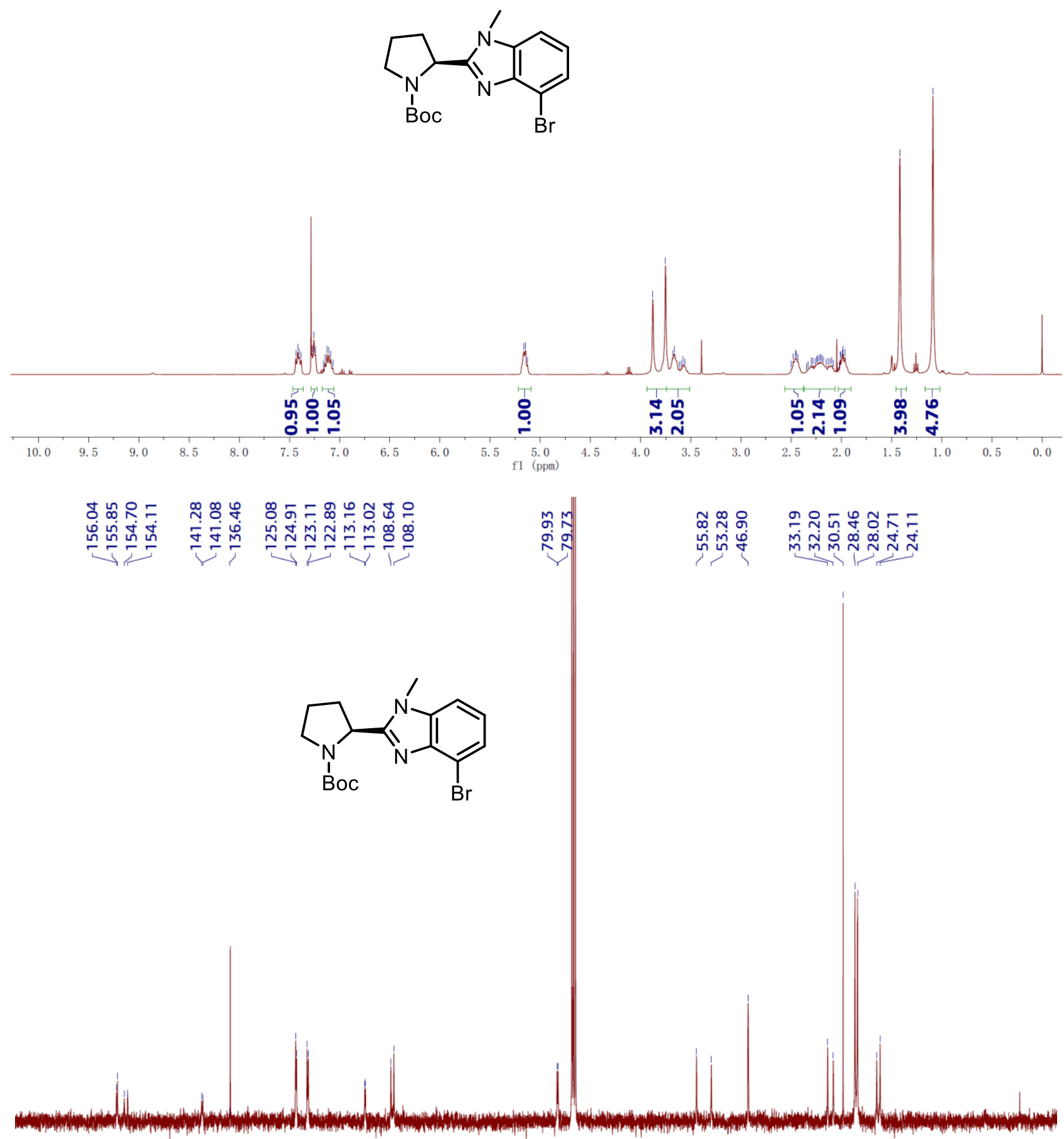

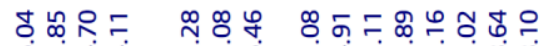

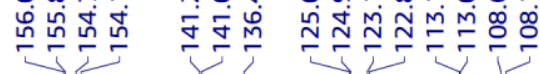
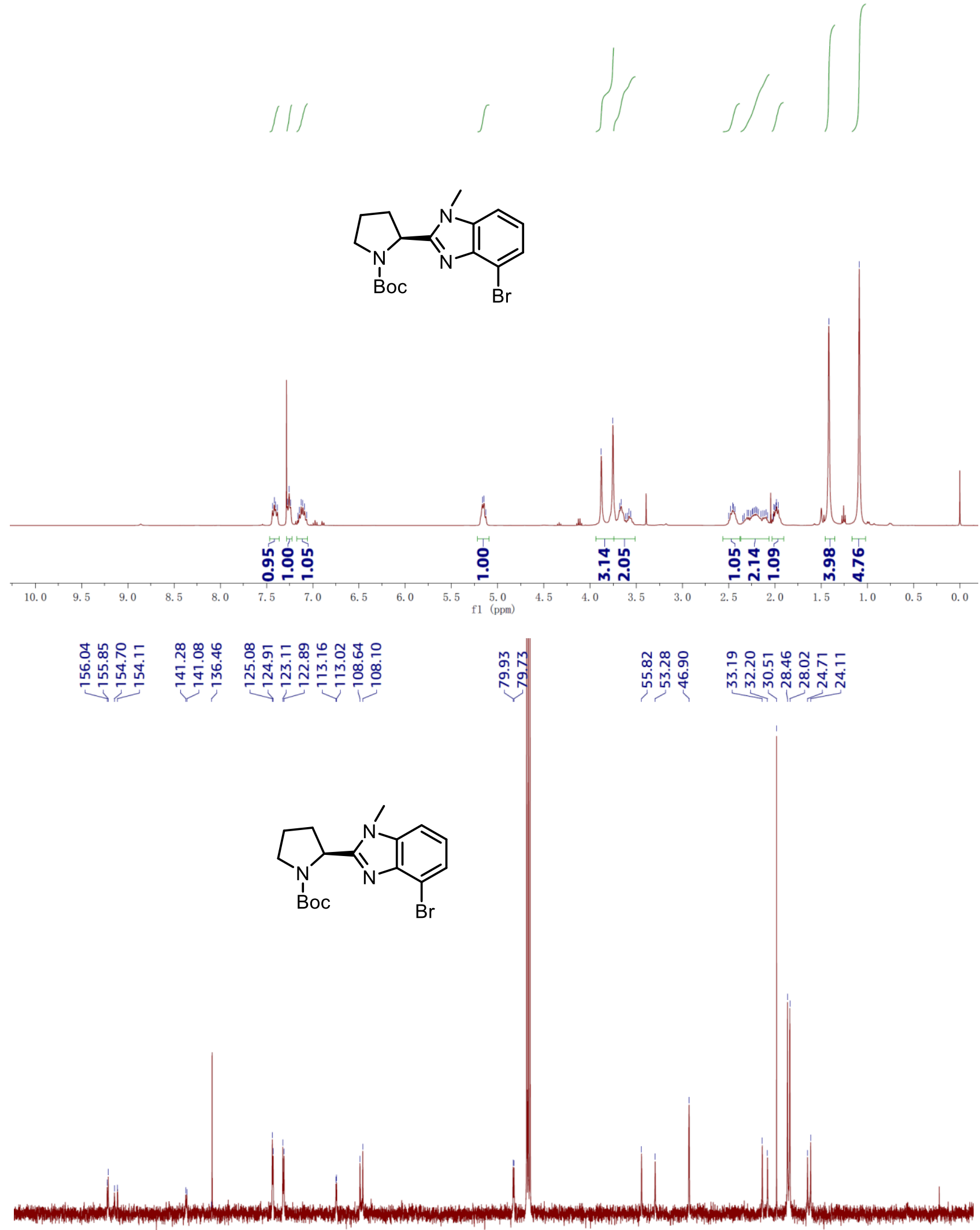

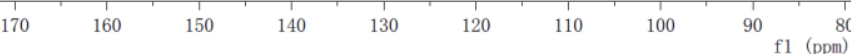


t 我
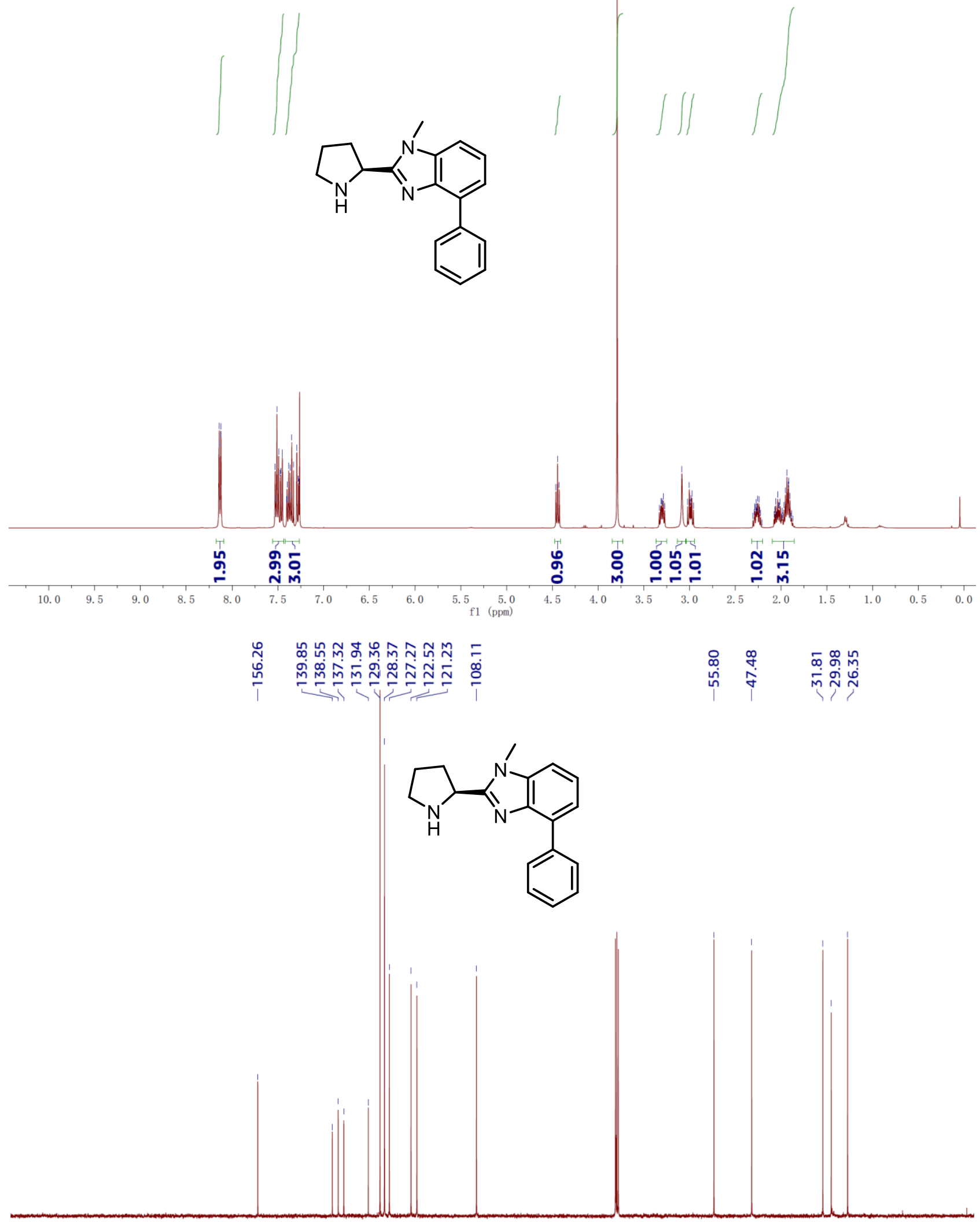
Nㅜำ

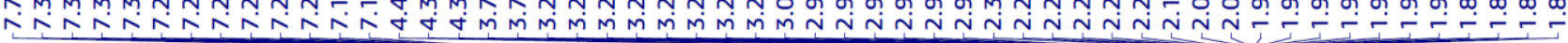<smiles>COc1c(C)cc(-c2cccc3c2nc(C2CCCN2)n3C)cc1C</smiles>

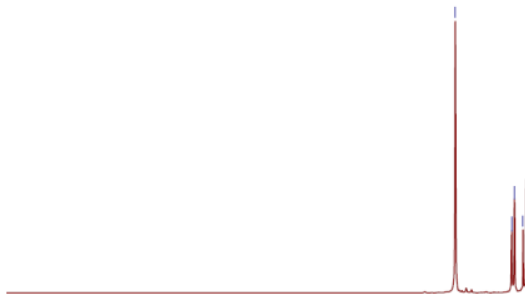

wit

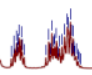

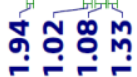

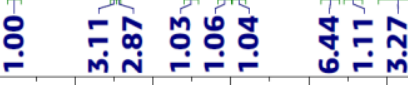

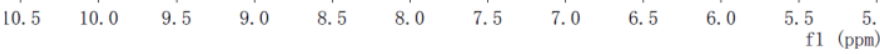

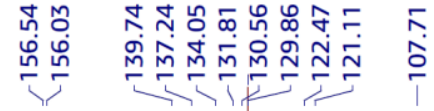

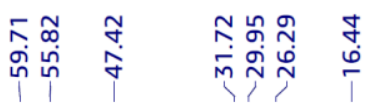<smiles>COc1c(C)cc(-c2cccc3c2nc(C2CCCN2)n3C)cc1C</smiles>

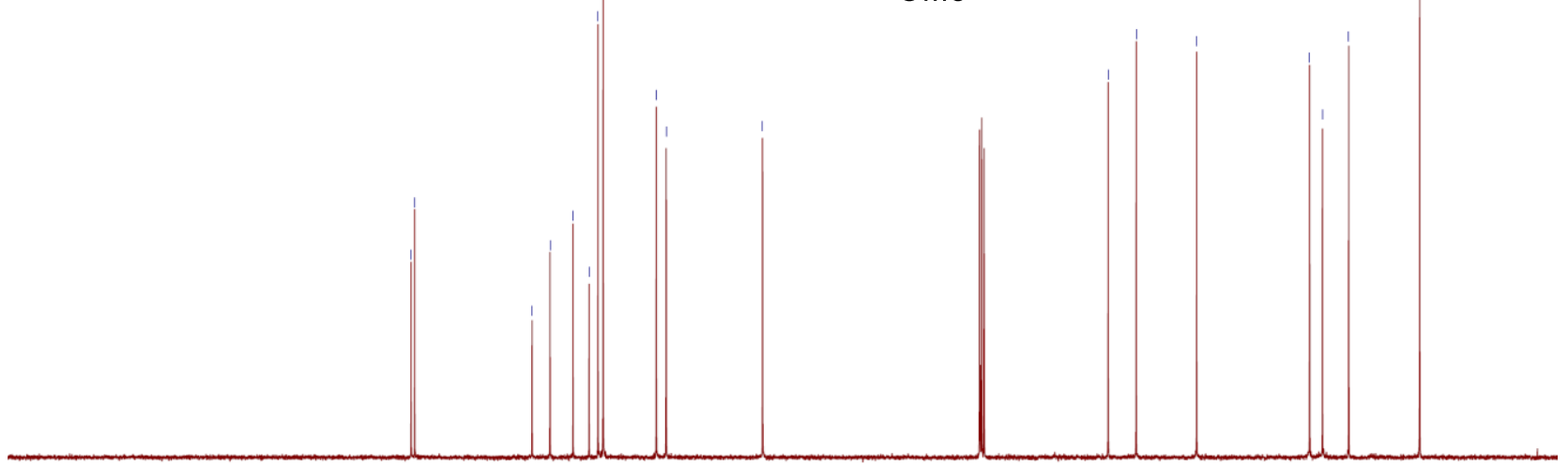

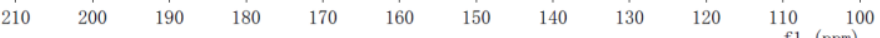

90

$80 \quad 70$

60

1 40 
<smiles>Cn1c(C2CCCN2)nc2c(-c3cc(C(C)(C)C)cc(C(C)(C)C)c3)cccc21</smiles>

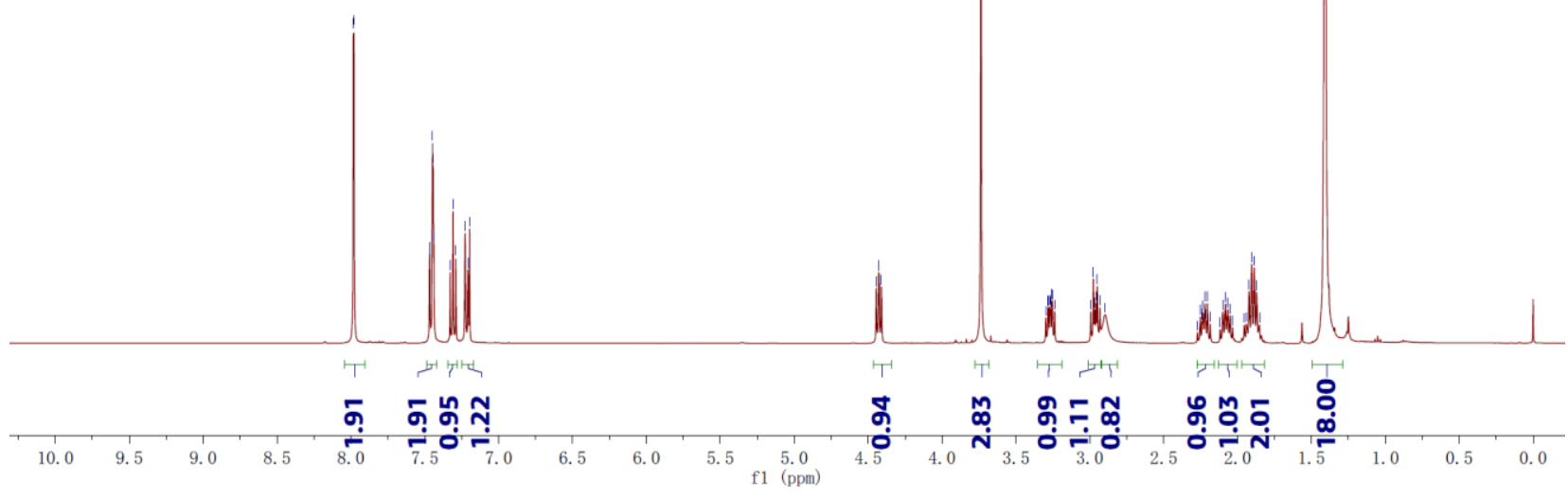

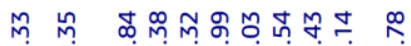

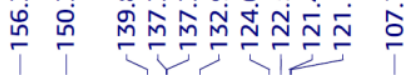
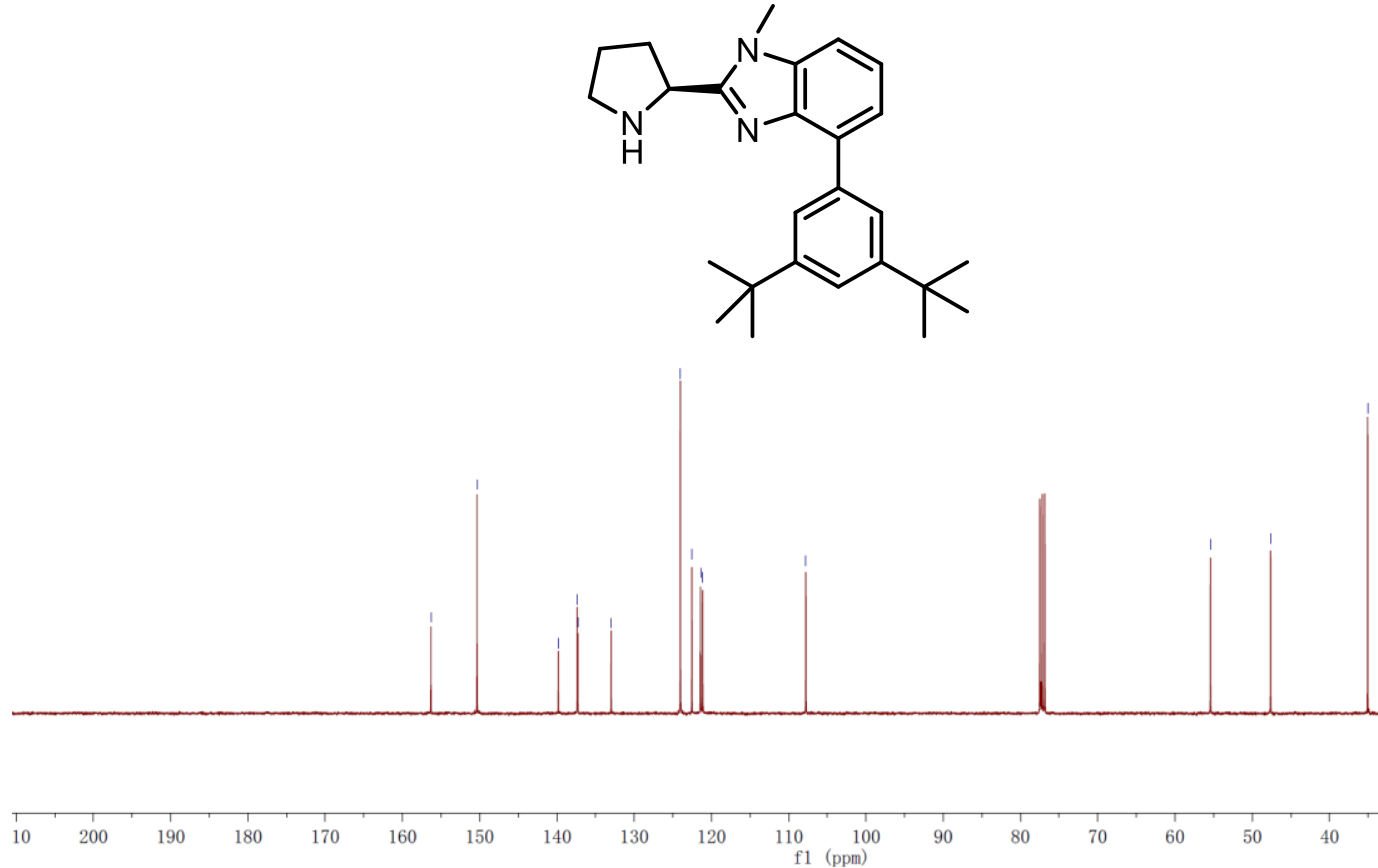
(4-bromo-1-methyl-1H-benzo[d]imidazol-2-yl)methanol (B1) Mำ

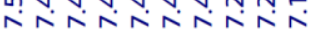

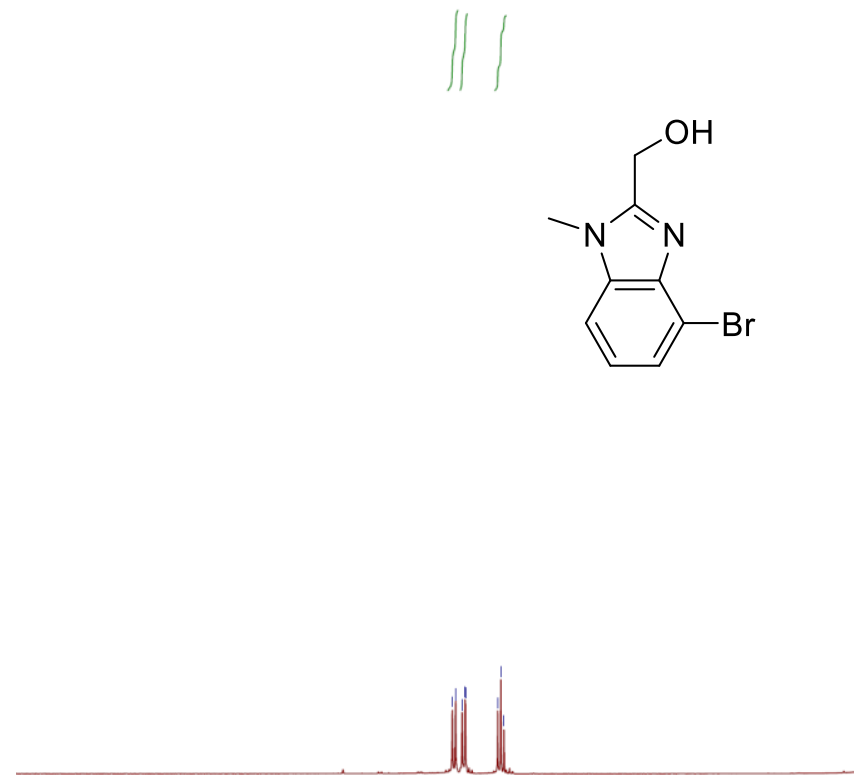

$\begin{array}{ll}\infty & \infty \\ \infty & \infty \\ \dot{\sigma} & \tilde{m} \\ \dot{1} & 1\end{array}$

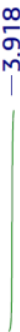

(2)

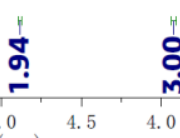

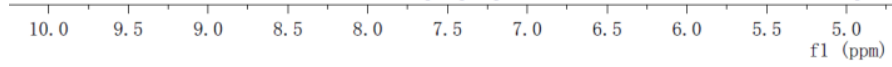

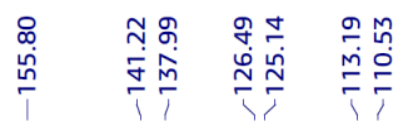

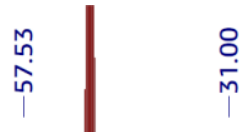

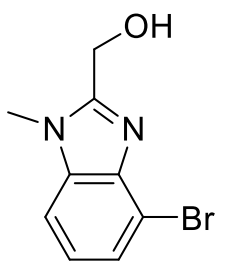

$\begin{array}{rrrrrrrrrrr}210 & 200 & 190 & 180 & 170 & 160 & 150 & 140 & 130 & 120 & 110 \\ \mathrm{fl} & \begin{array}{r}100 \\ (\mathrm{ppm})\end{array}\end{array}$ 

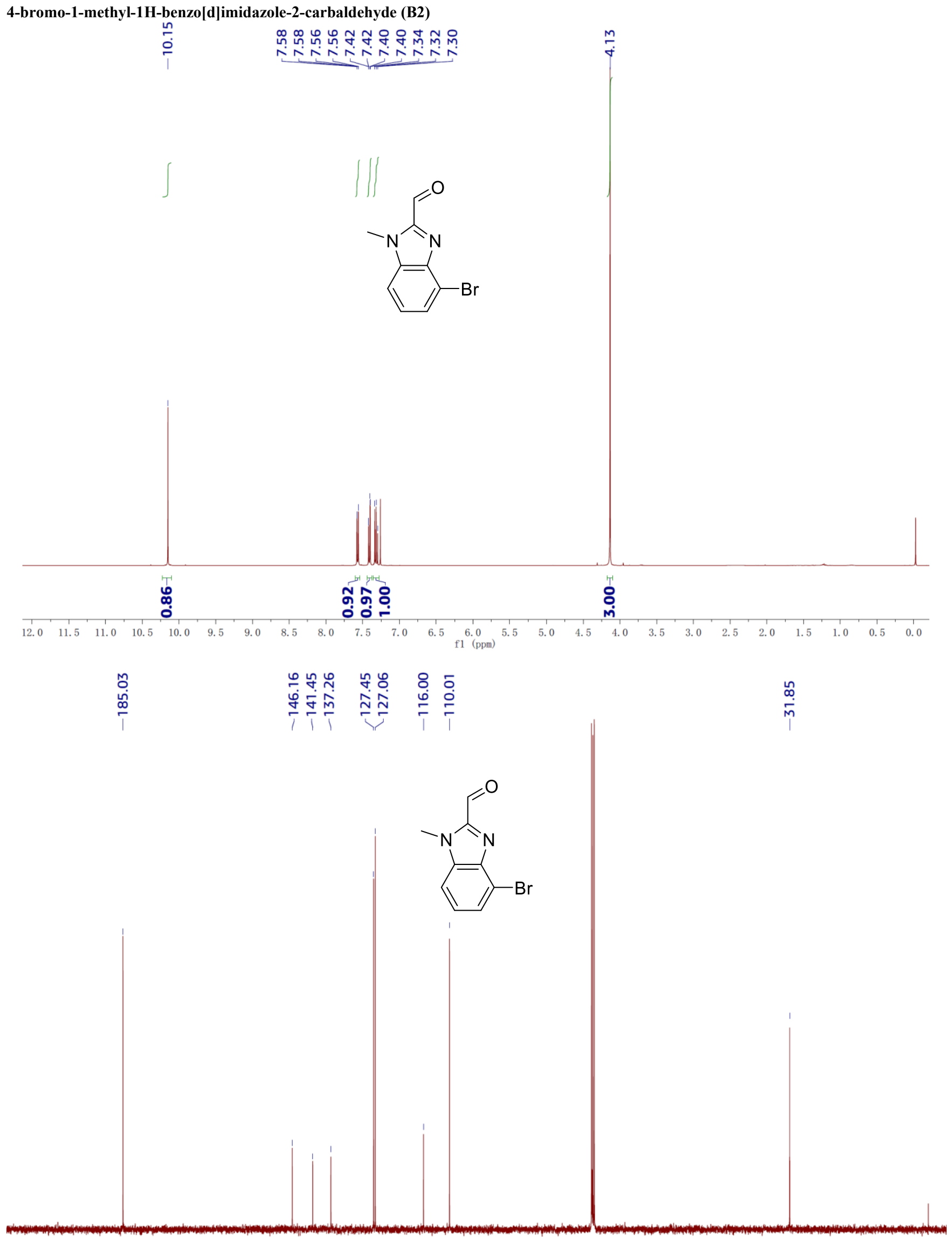
1-methyl-4-phenyl-1H-benzo[d]imidazole-2-carbaldehyde (B3)

กั

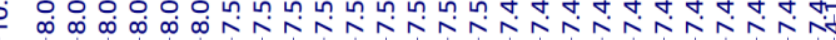

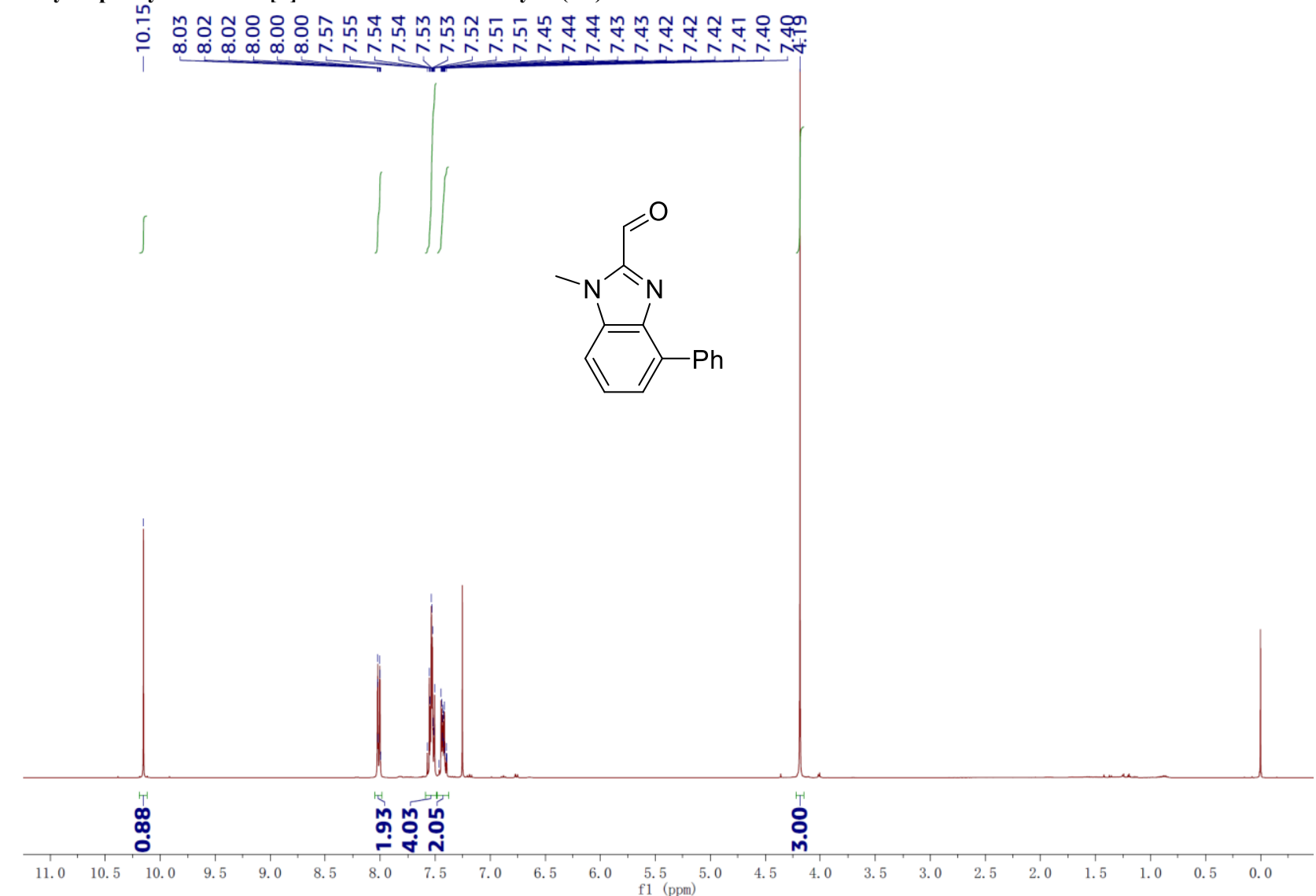

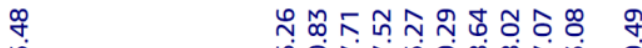

虫

$\frac{\hat{f}}{\hat{m}}$

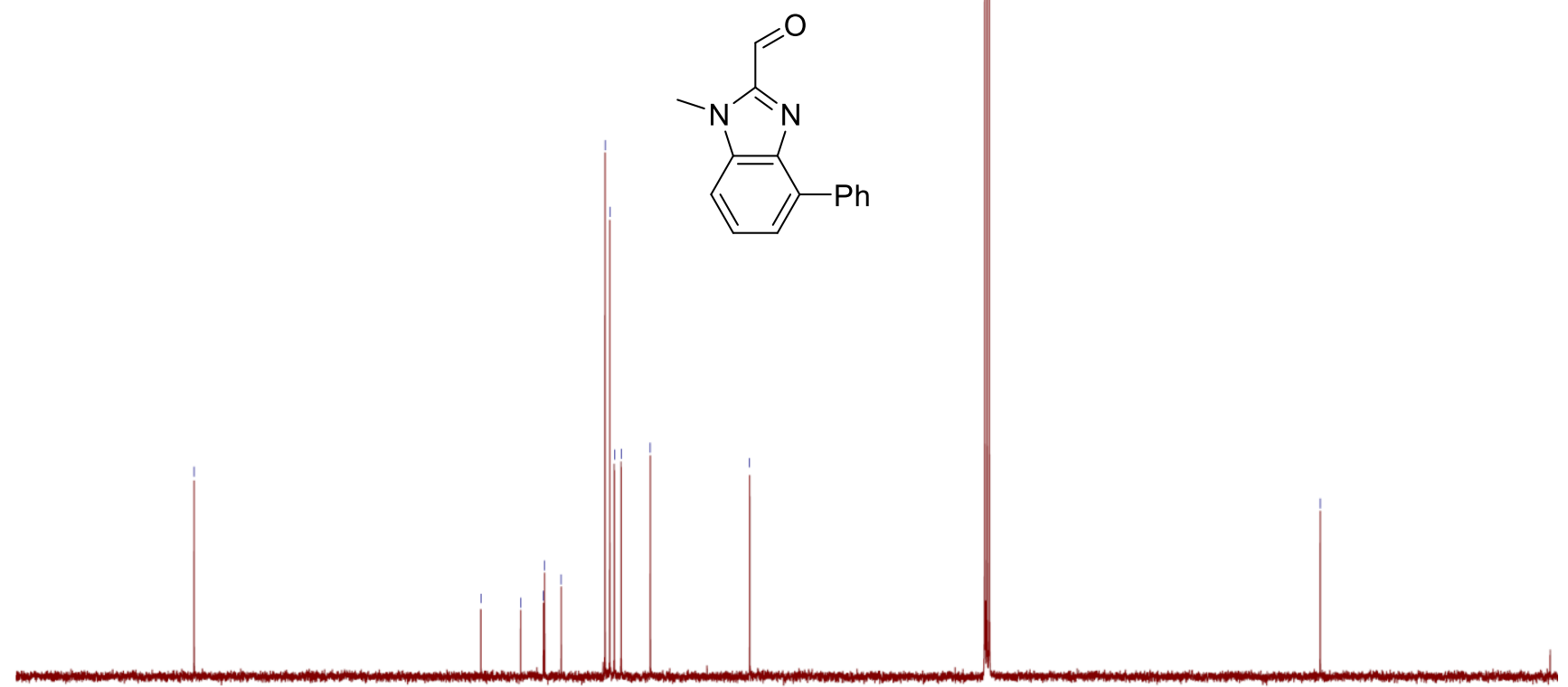

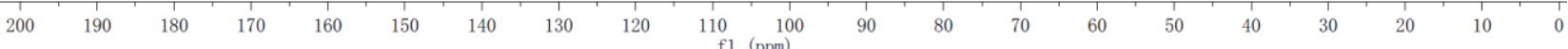


(S,E)-2-methyl-N-((1-methyl-4-phenyl-1H-benzo[d]imidazol-2-yl)methylene)propane-2-sulfinamide (B4)

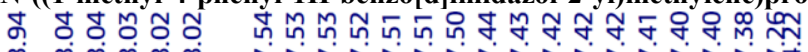

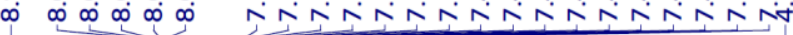

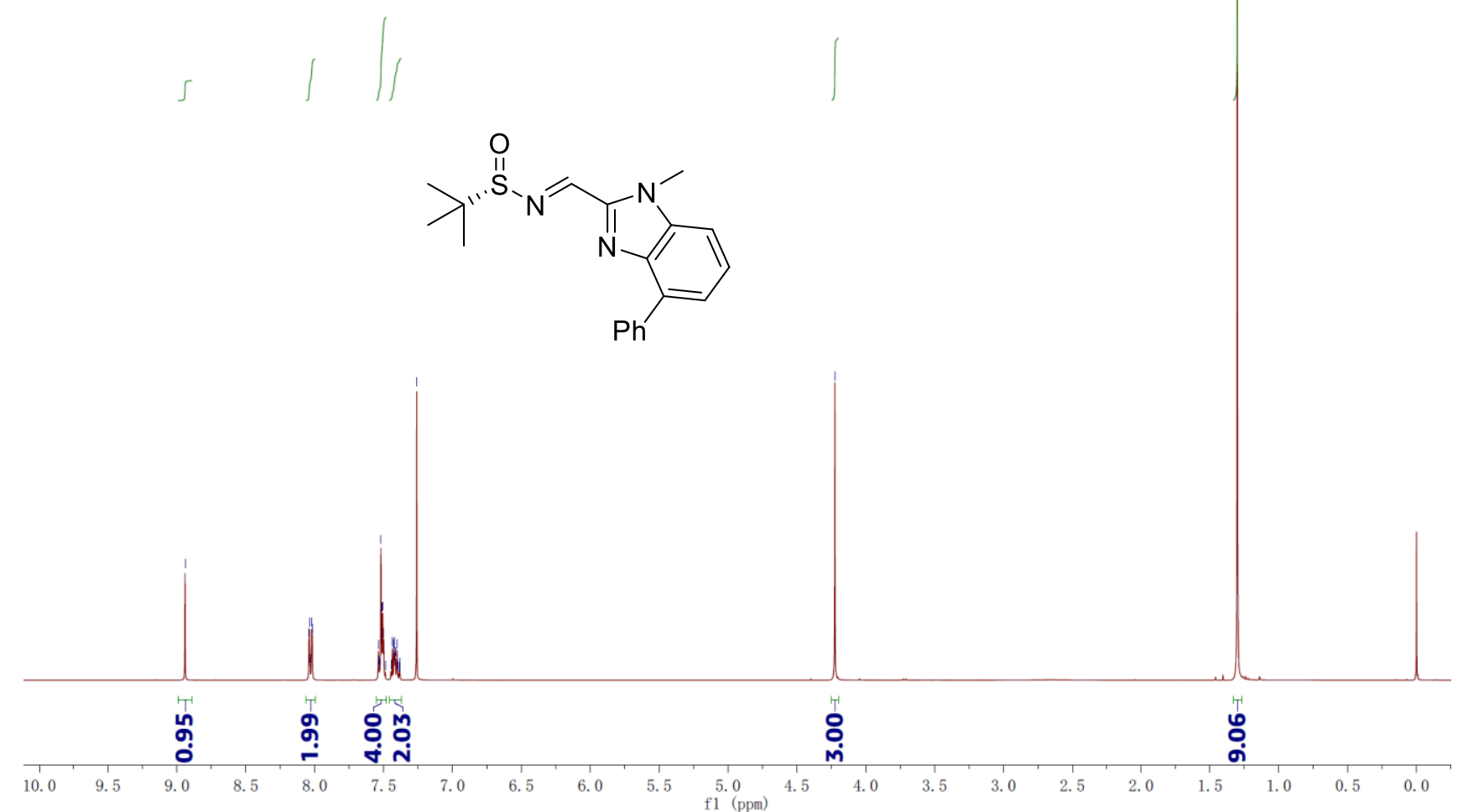

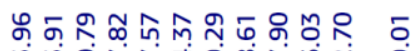

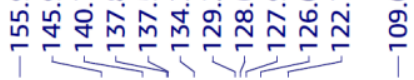

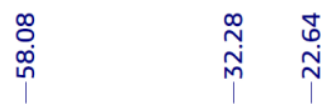<smiles>Cn1c(/C=N/[S@](=O)C(C)(C)C)nc2c(-c3ccccc3)cccc21</smiles>

$\begin{array}{llllllllll}200 & 190 & 180 & 170 & 160 & 150 & 140 & 130 & 120 & 110 \\ \mathrm{f} 1(\mathrm{ppm})\end{array}$ 
(S)-N-((S)-2,2-dimethyl-1-(1-methyl-4-phenyl-1H-benzo[d]imidazol-2-yl)propyl)-2-methylpropane-2-sulfinamide (B5)

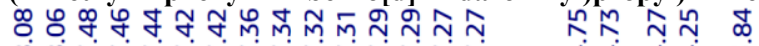

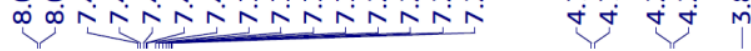<smiles>Cn1c([C@H](NS(=O)C(C)(C)C)C(C)(C)C)nc2c(-c3ccccc3)cccc21</smiles>

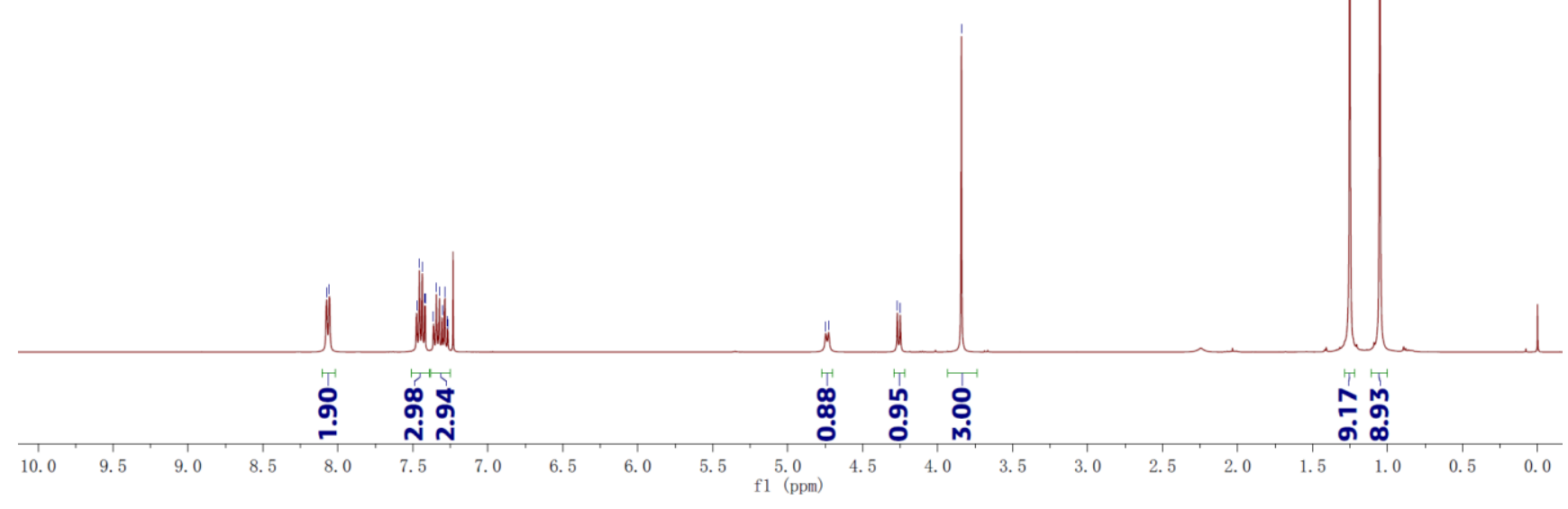

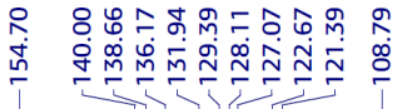

ஸ़ธ

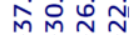

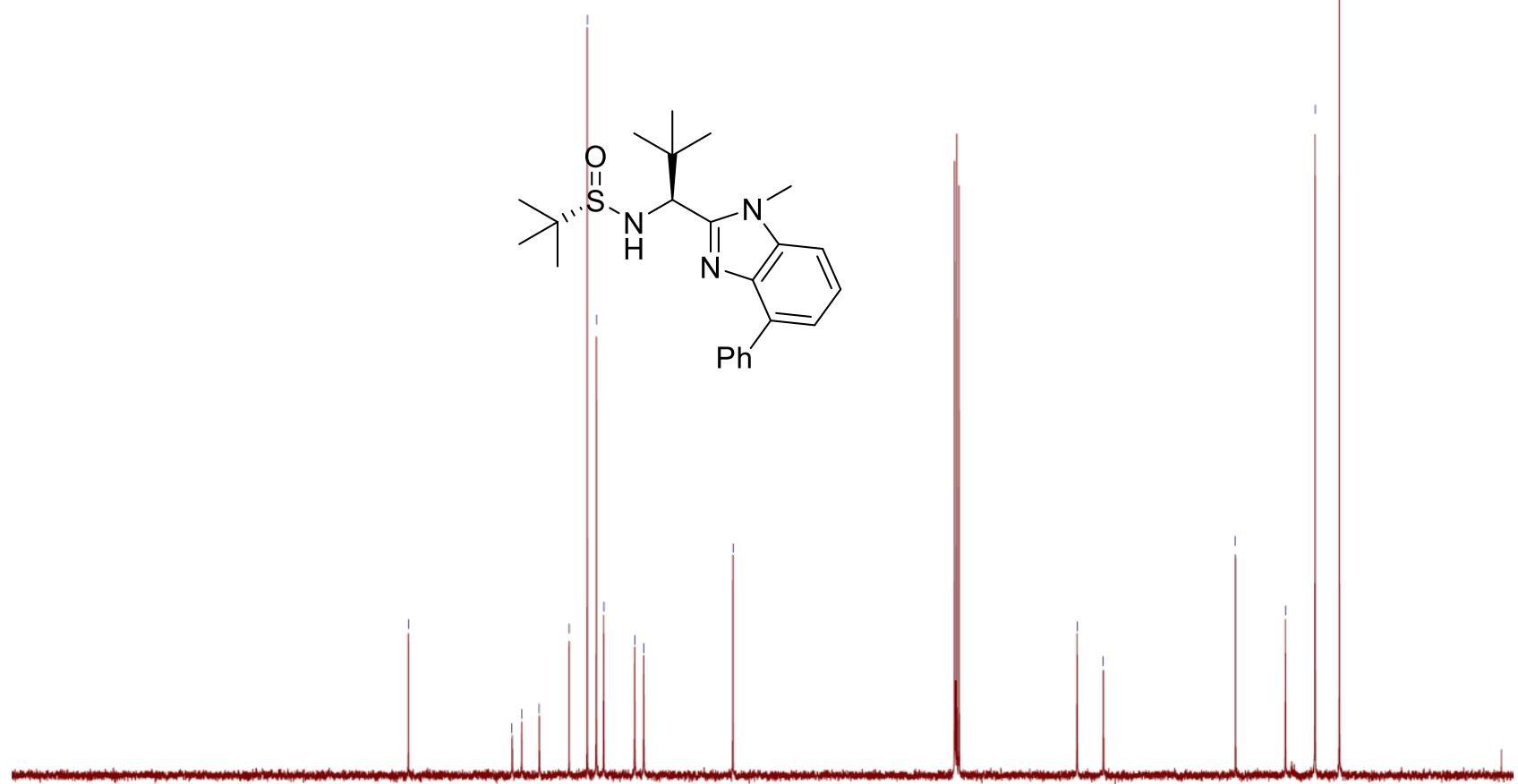


(S)-2,2-dimethyl-1-(1-methyl-4-phenyl-1H-benzo[d]imidazol-2-yl)propan-1-amine (L9)

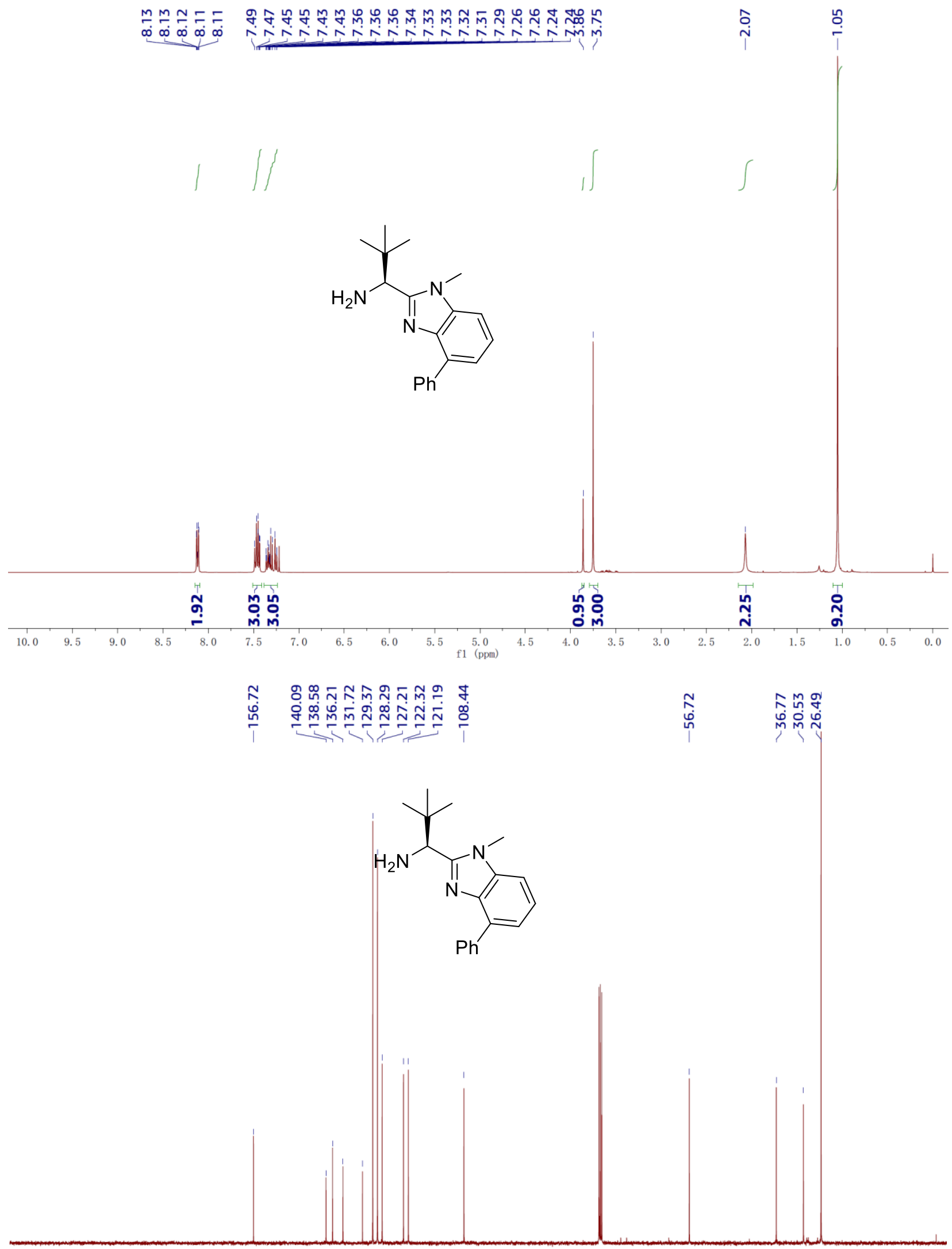

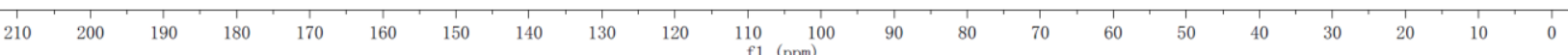


Mn-1

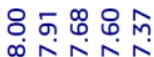

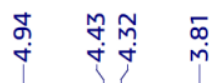

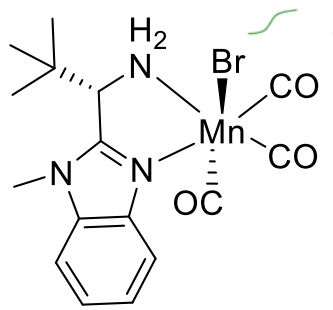

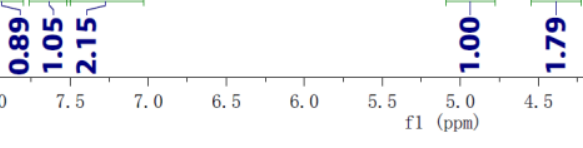

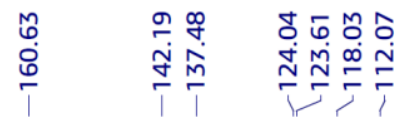

ำ

ळ $m$ m

NNN

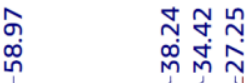

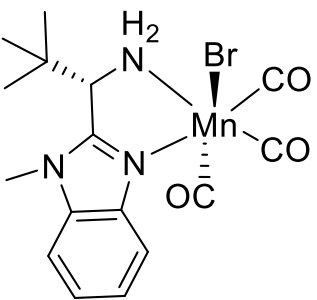


Mn-2

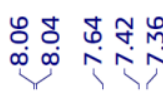

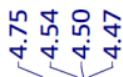

$>^{\prime \prime .} \mathrm{N}^{\mathrm{N}} \longrightarrow \mathrm{Br}$

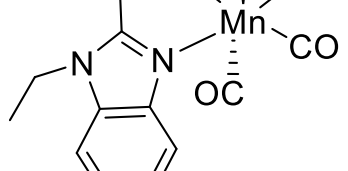

11

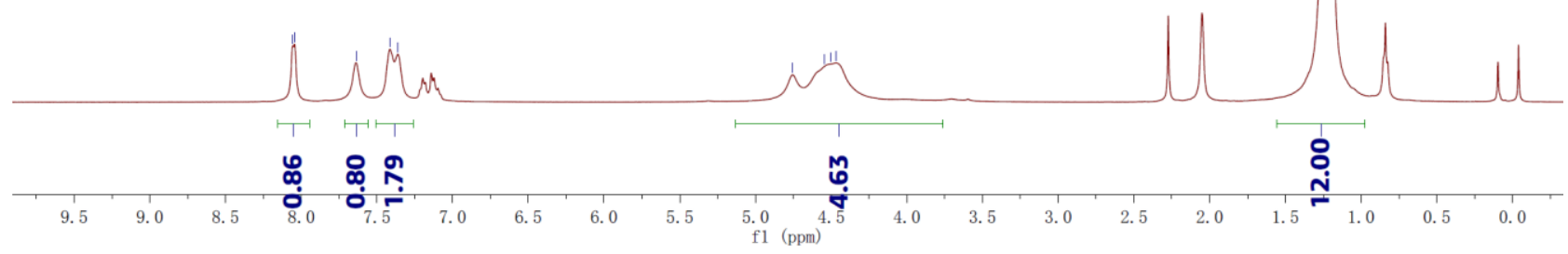

นึํำ

$\sqrt{n} \underset{n}{n}$

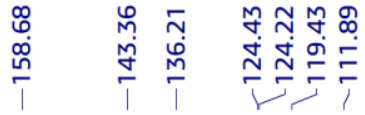

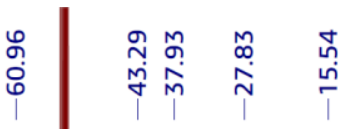

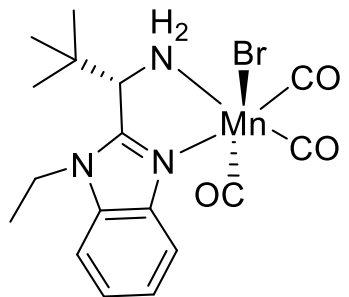

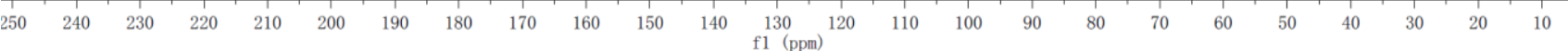




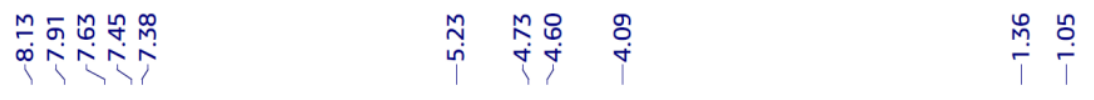
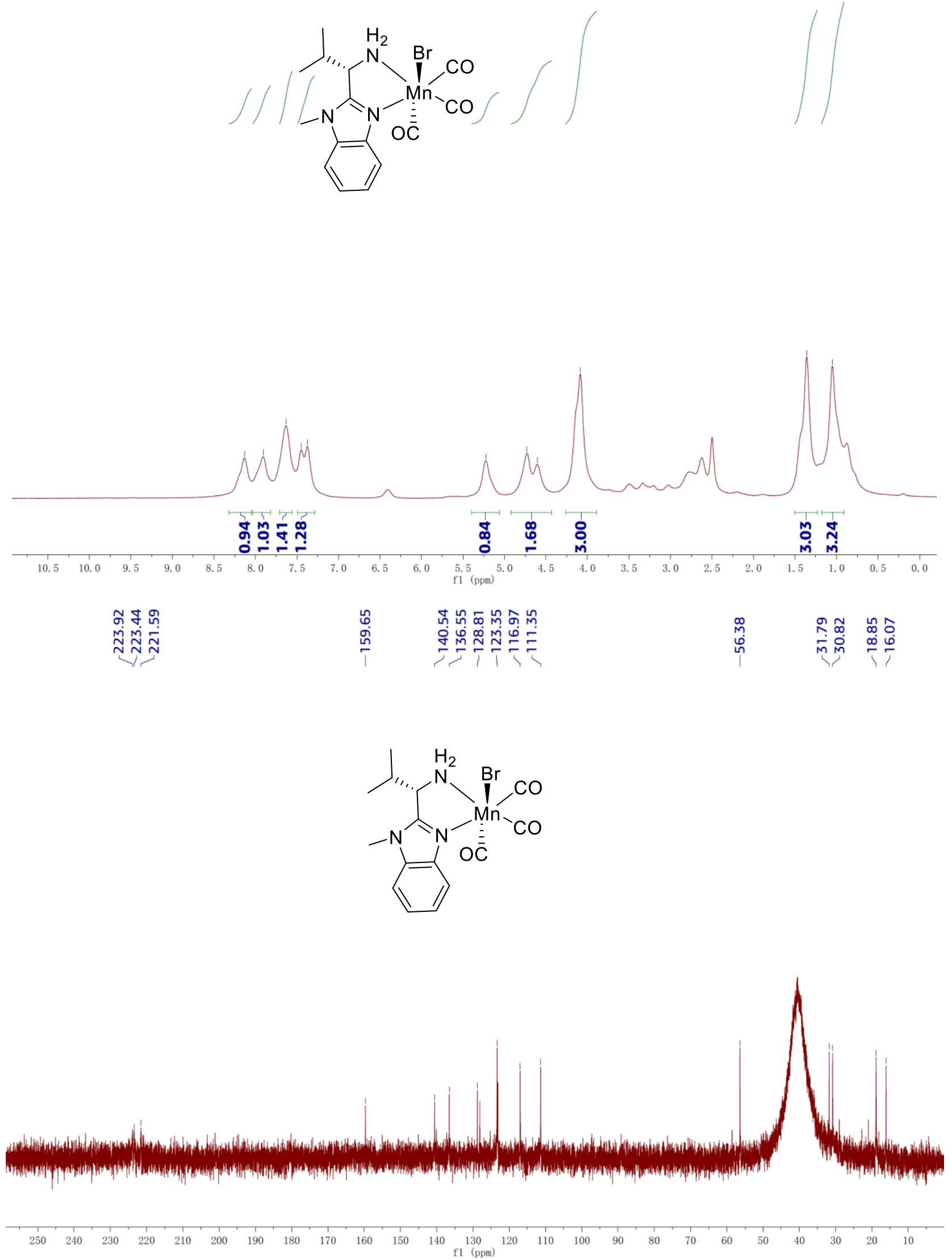
Mn-4

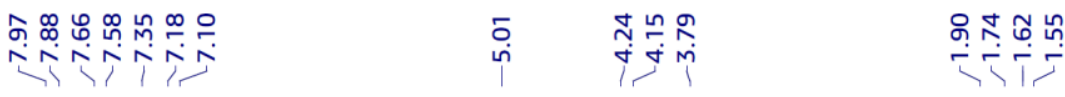
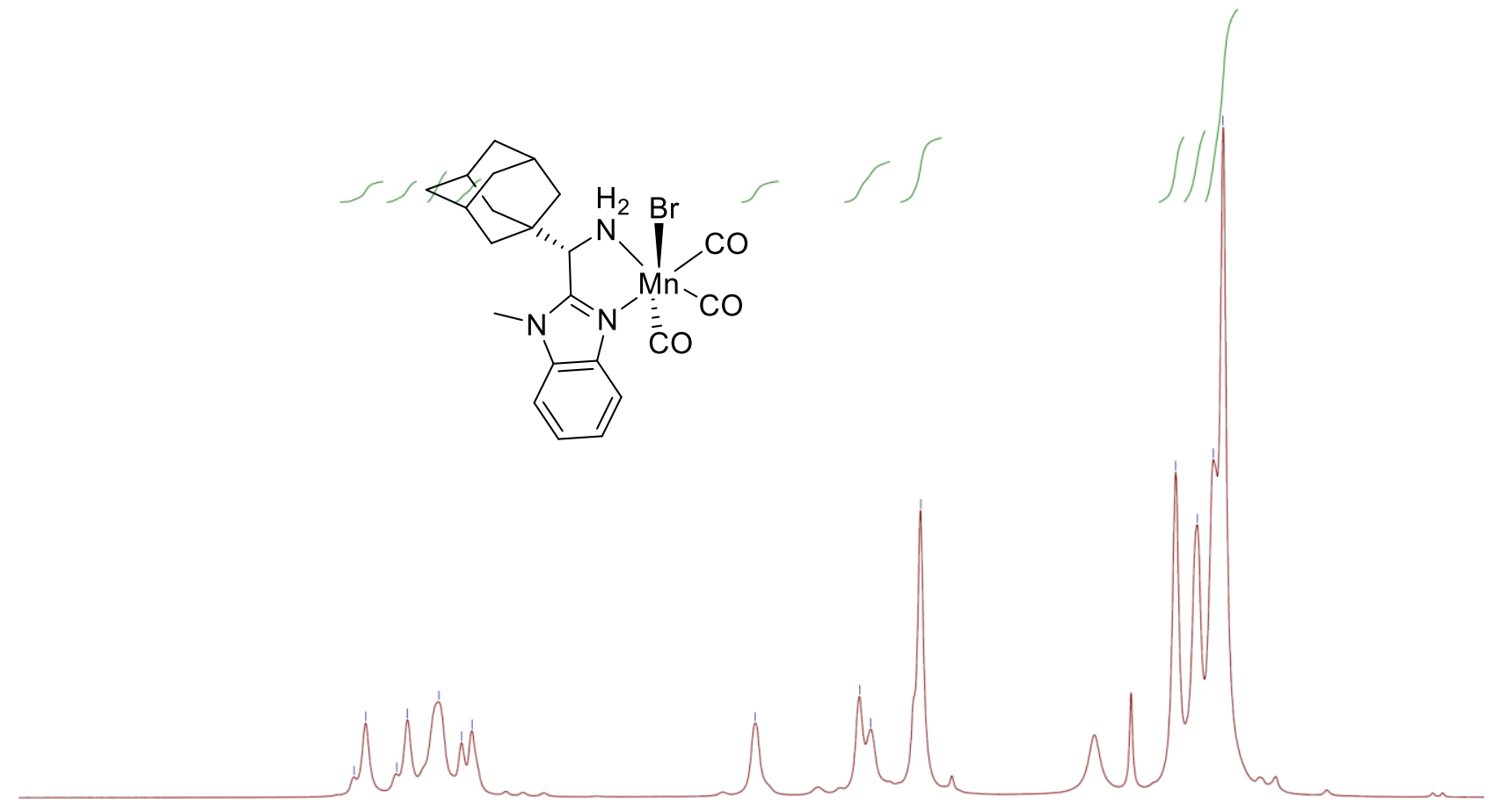

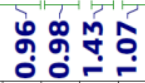

ลิ

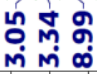

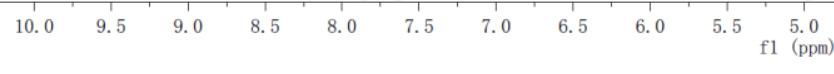

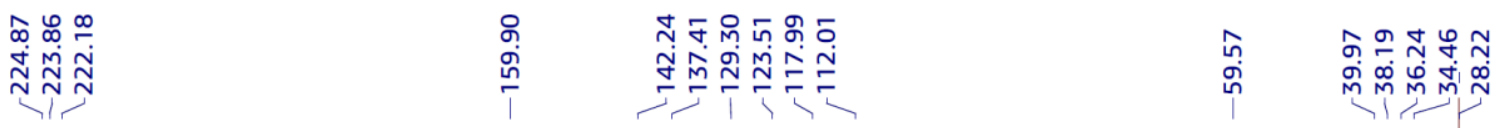

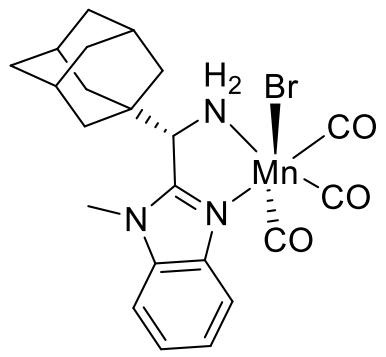

$\begin{array}{rrrrrrrrrrrrr}240 & 230 & 220 & 210 & 200 & 190 & 180 & 170 & 160 & 150 & 140 & 130 & 120 \\ \text { f1 (ppm) }\end{array}$ 
Mn-5

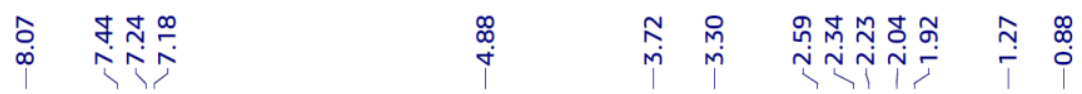
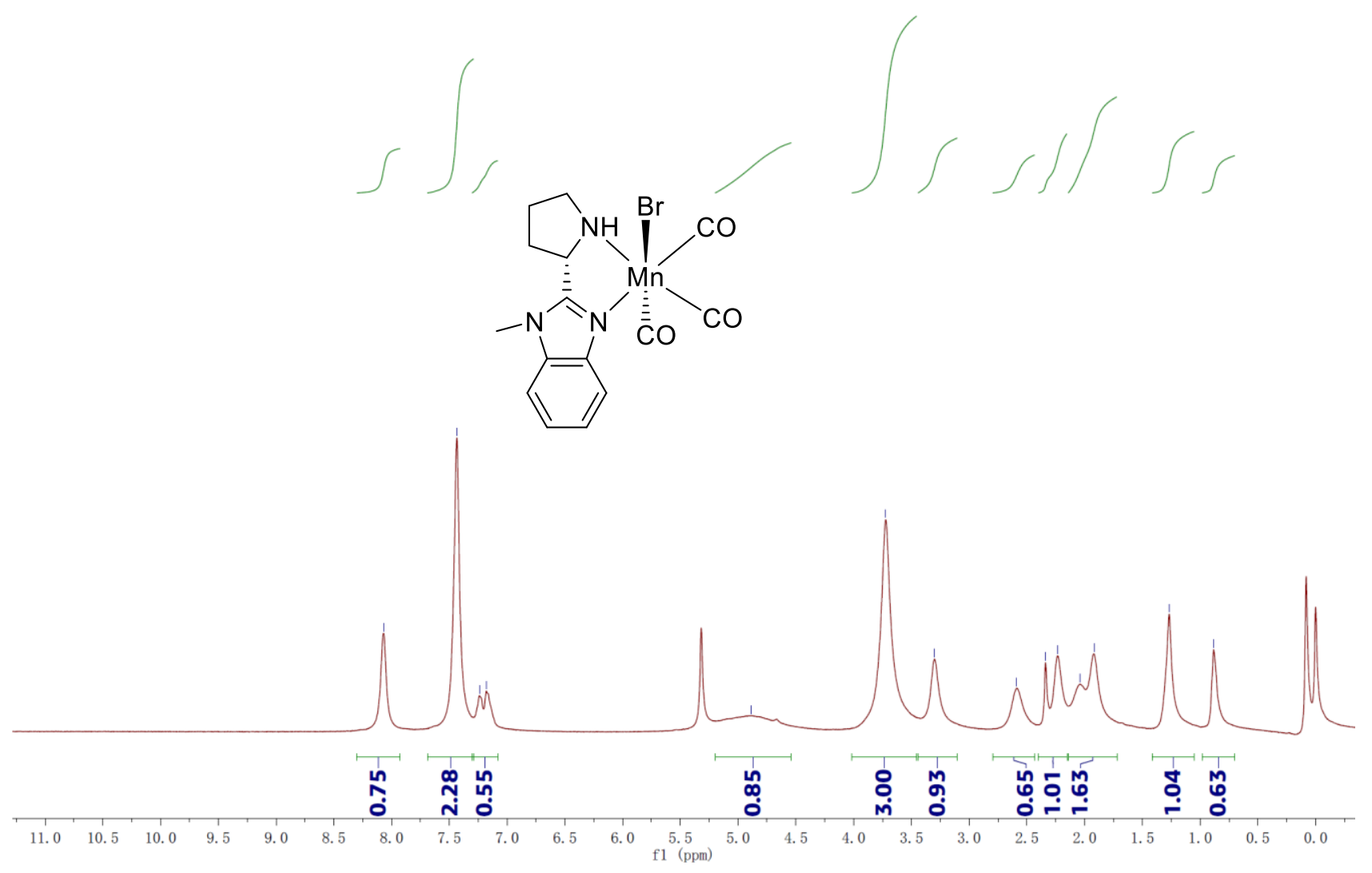

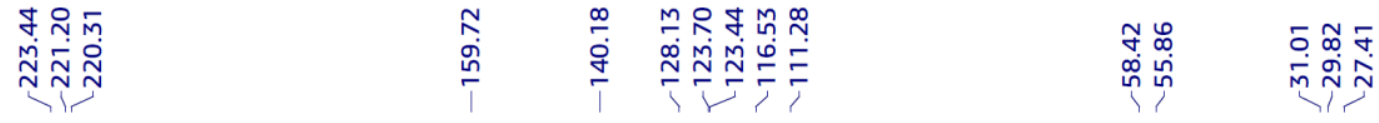
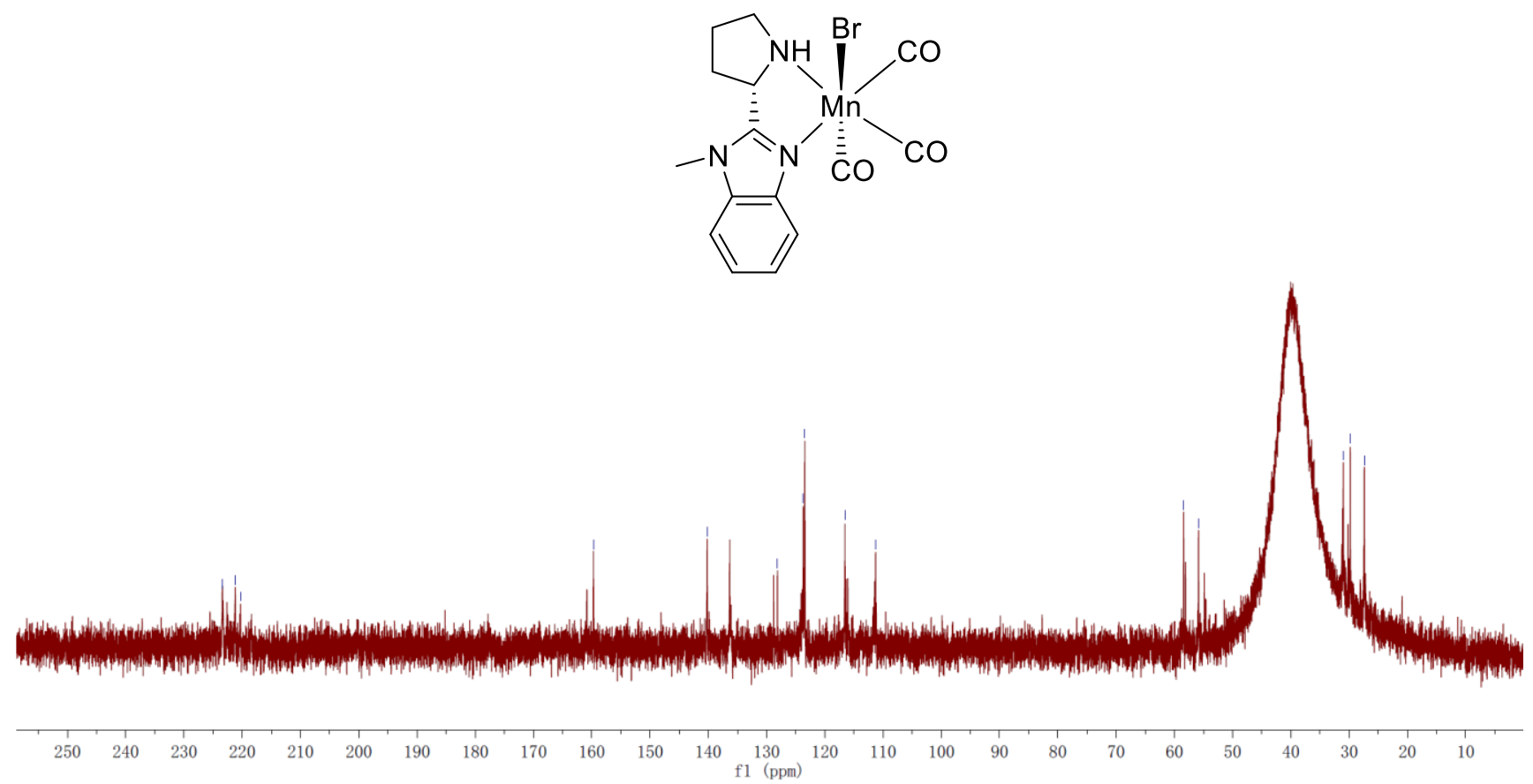
Mn-6
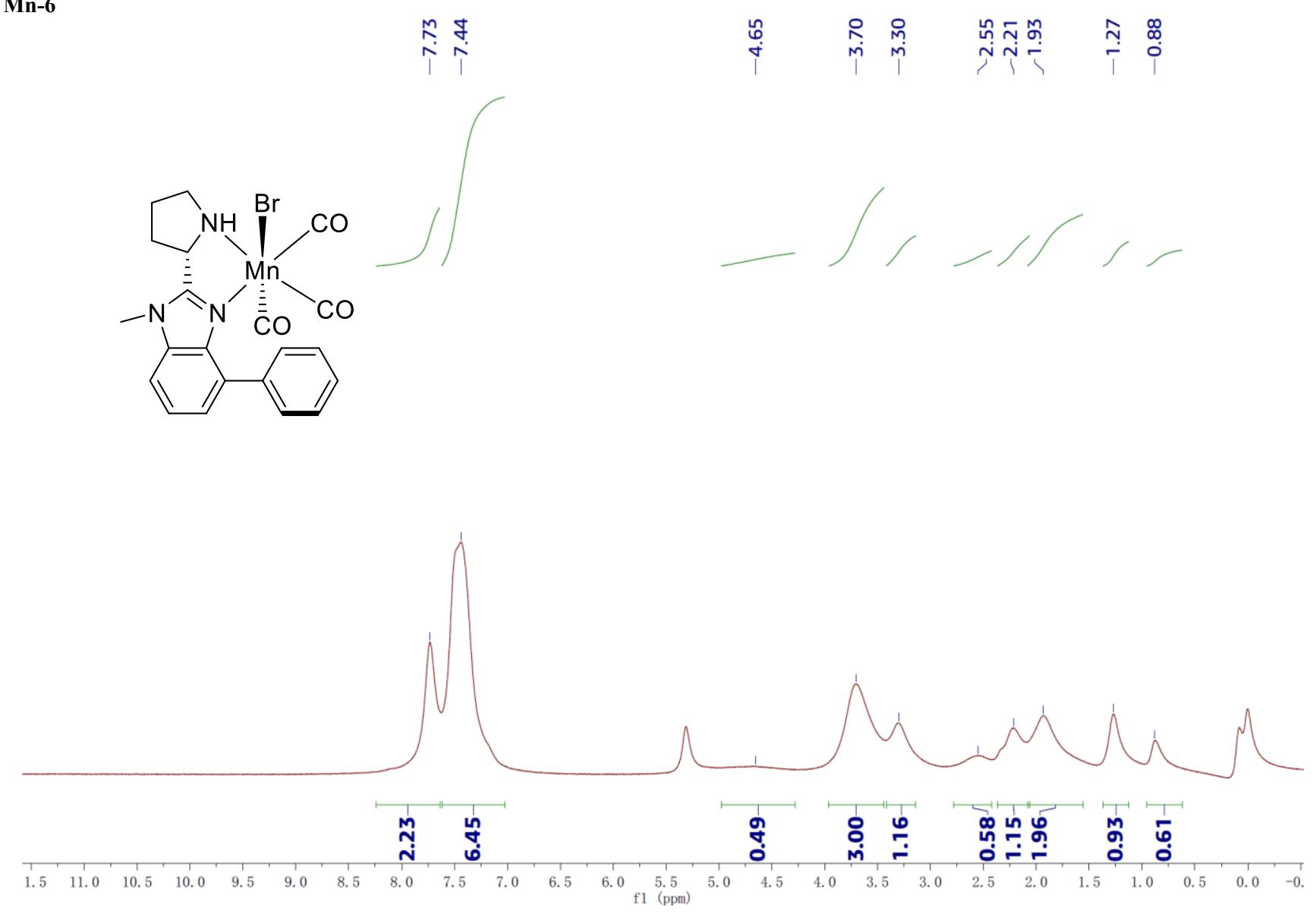

福曼
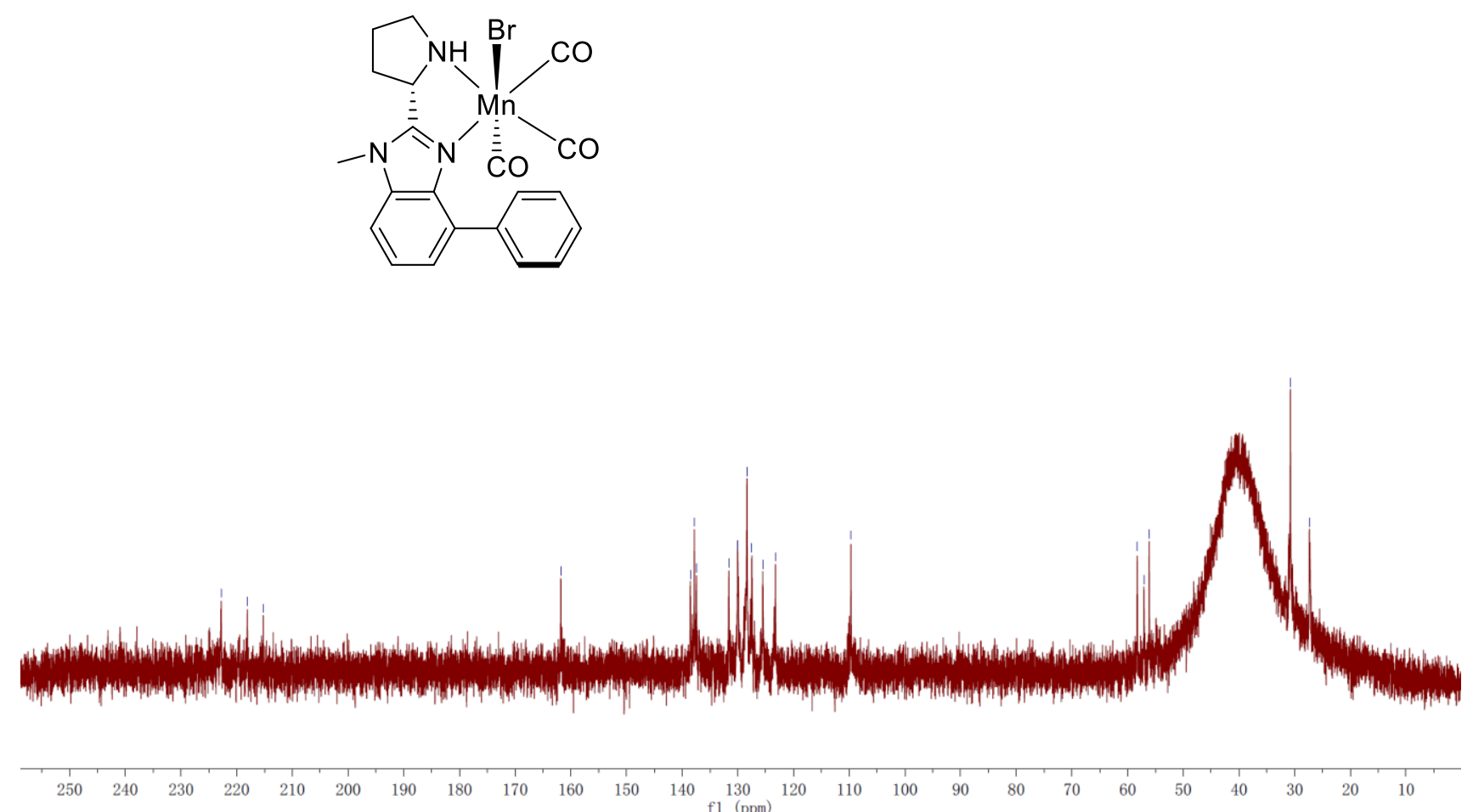
Mn-7
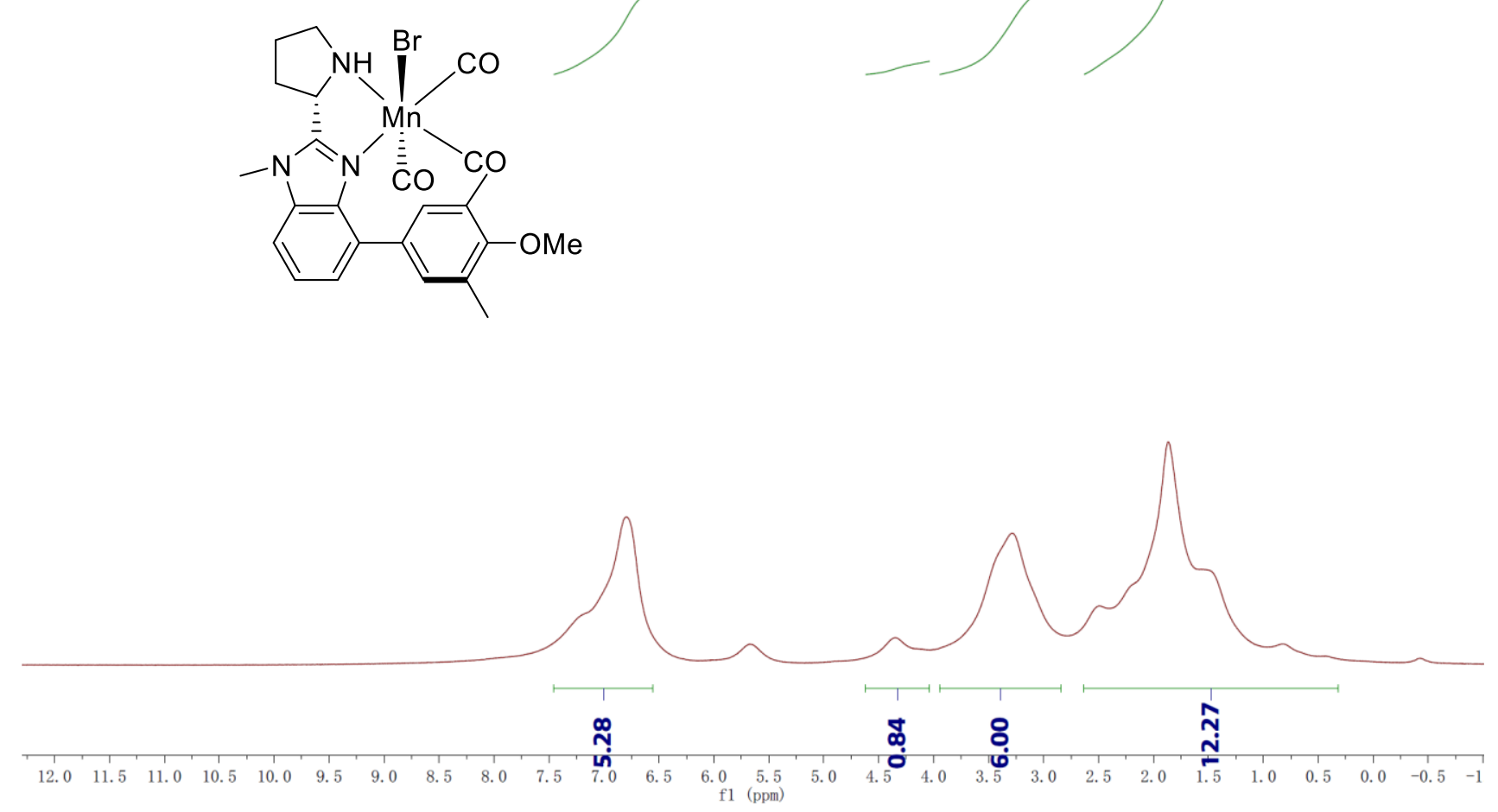

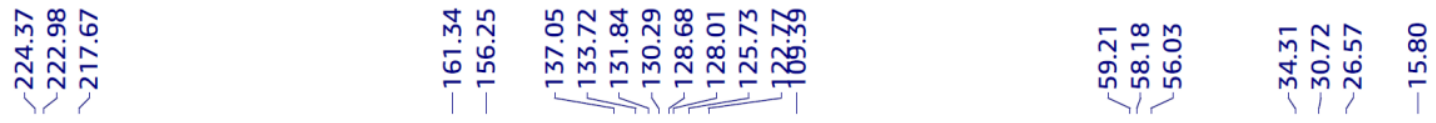

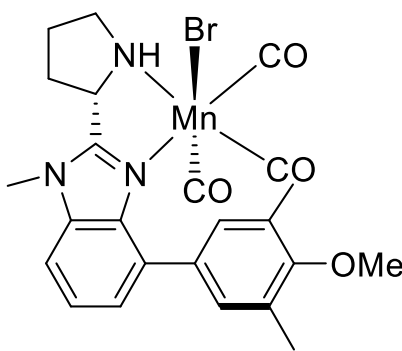

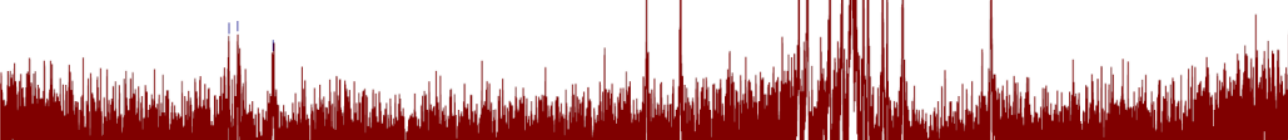

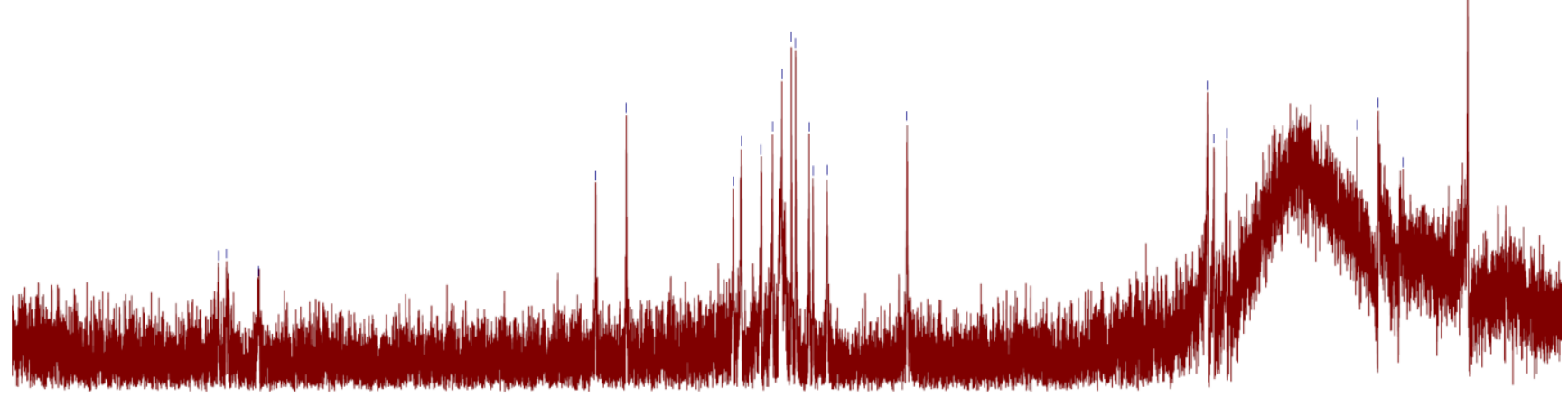

$\begin{array}{llllllllllllll}250 & 240 & 230 & 220 & 210 & 200 & 190 & 180 & 170 & 160 & 150 & 140 & 130 & 120 \\ \mathrm{f} 1(\mathrm{ppm}) & \end{array}$ 


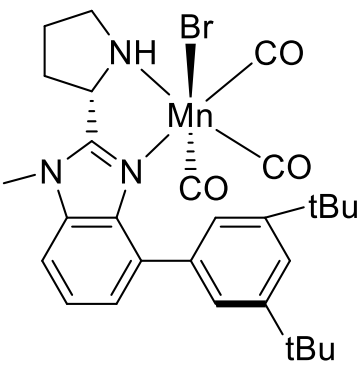

$\mathrm{Bu}$
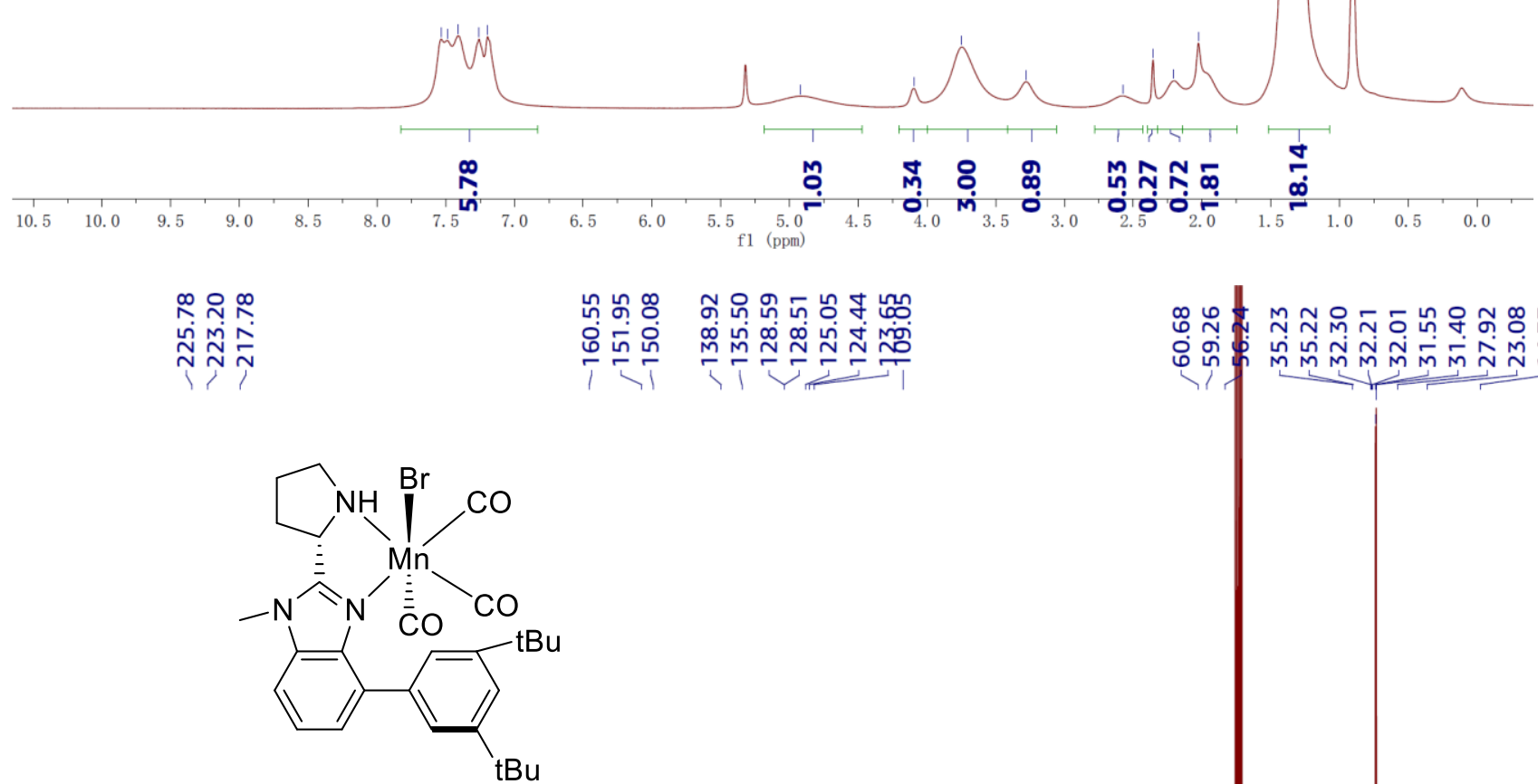

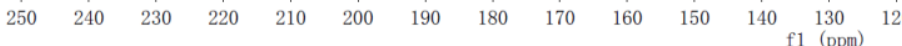

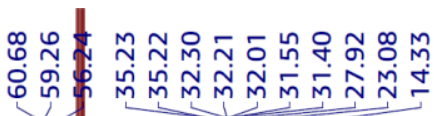


Mn-9

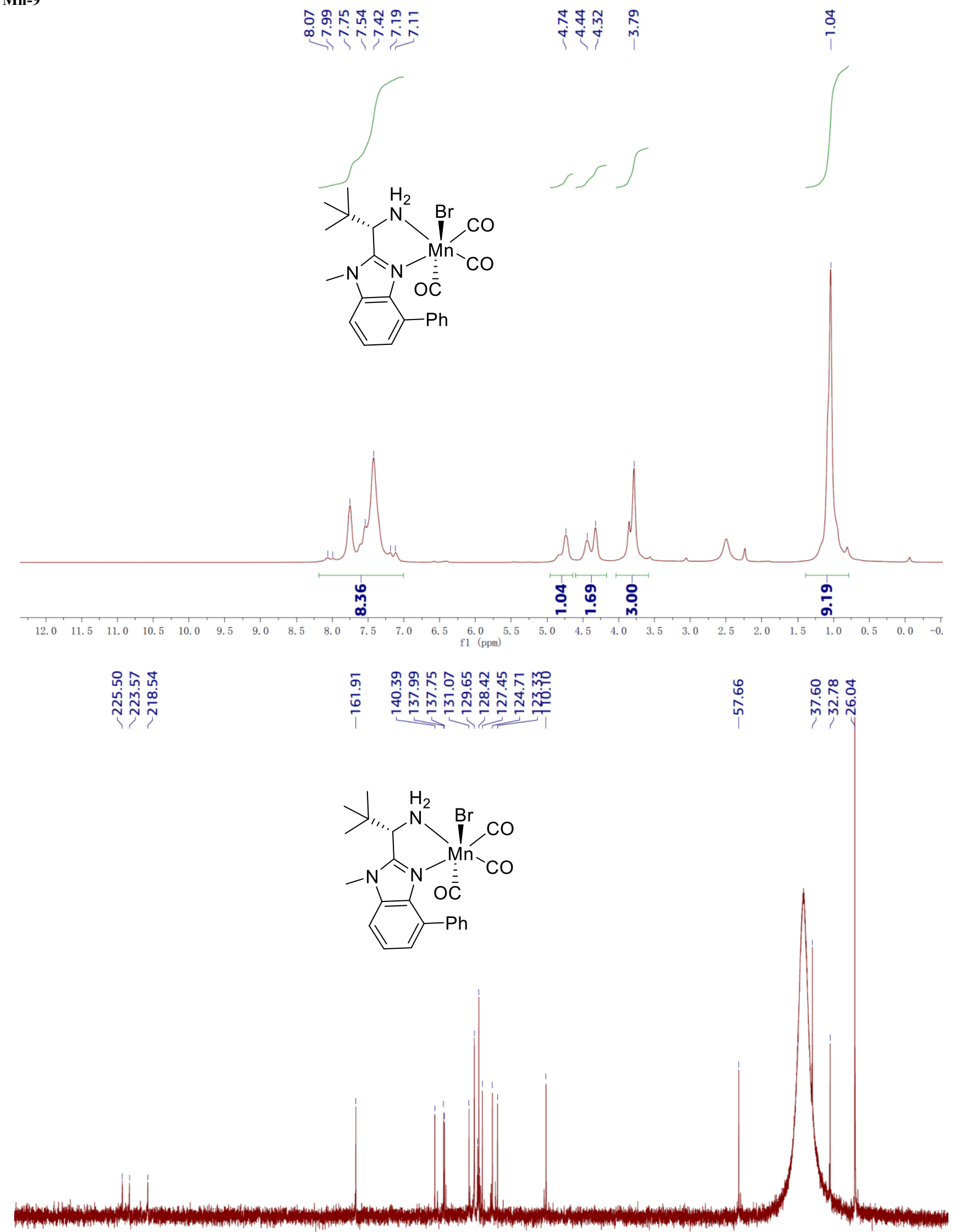

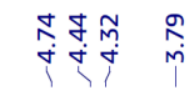

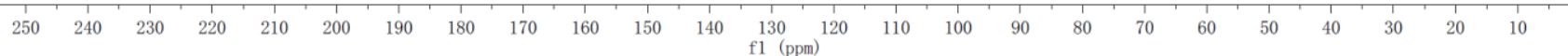

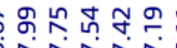

\section{.}


S)-1-phenylethan-1-ol (2a)
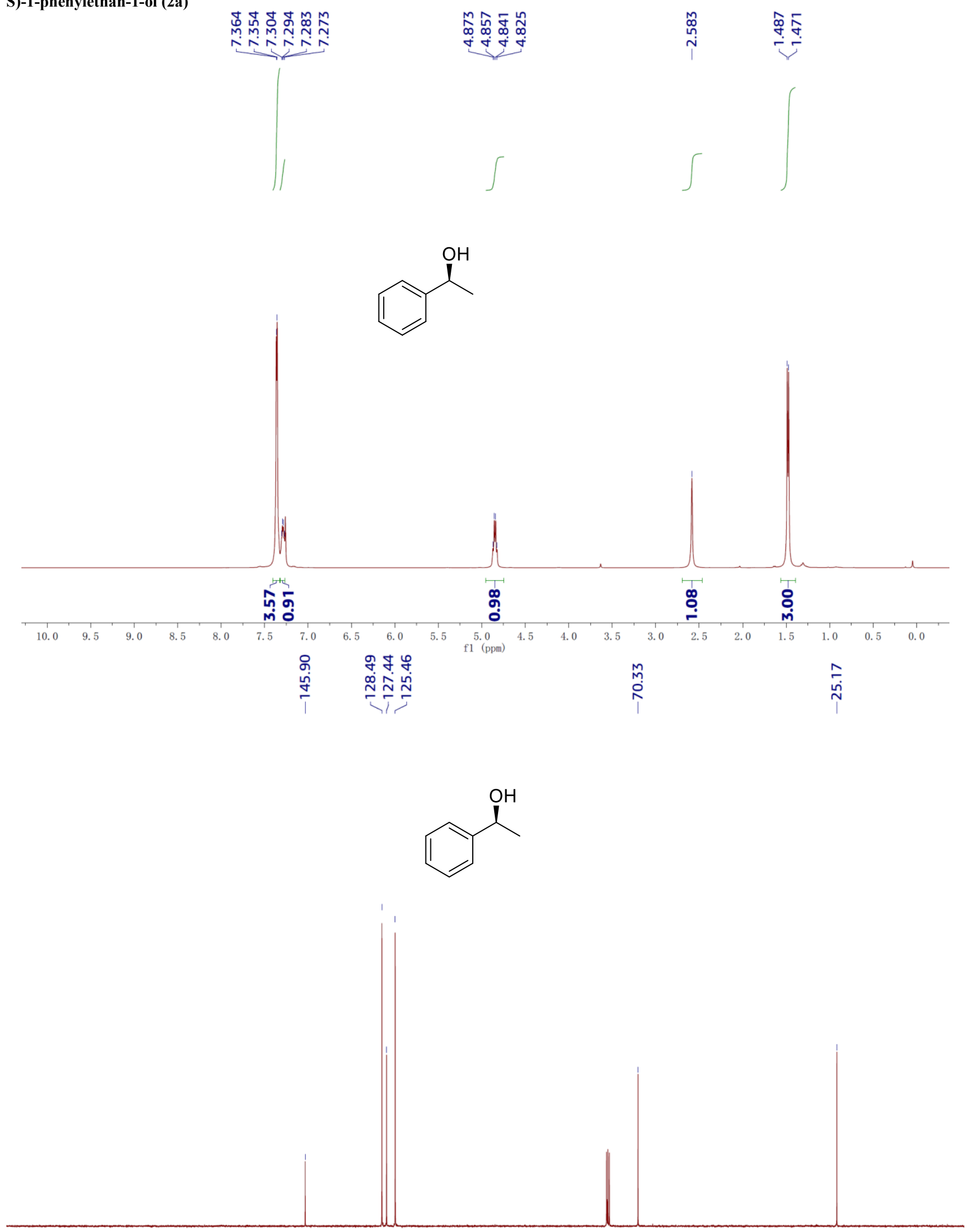

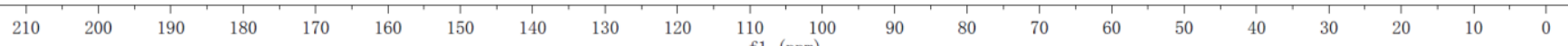


(S)-1-(o-tolyl)ethan-1-ol (2b)

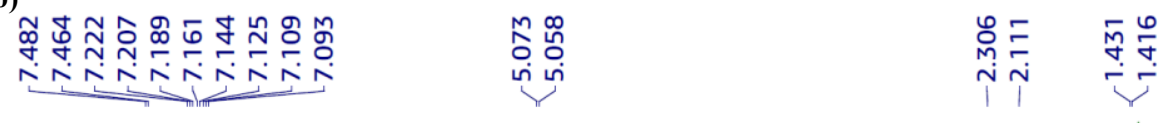

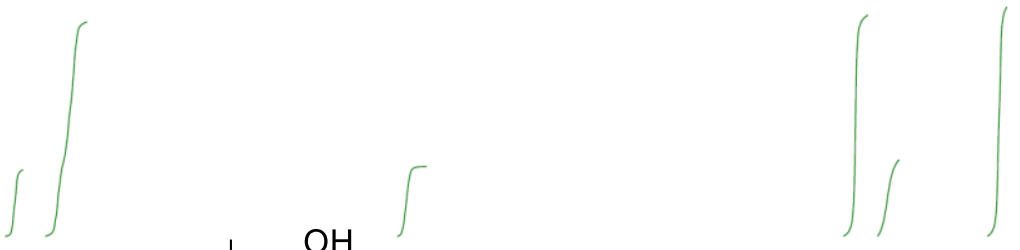<smiles>Cc1ccccc1[C@H](C)O</smiles>
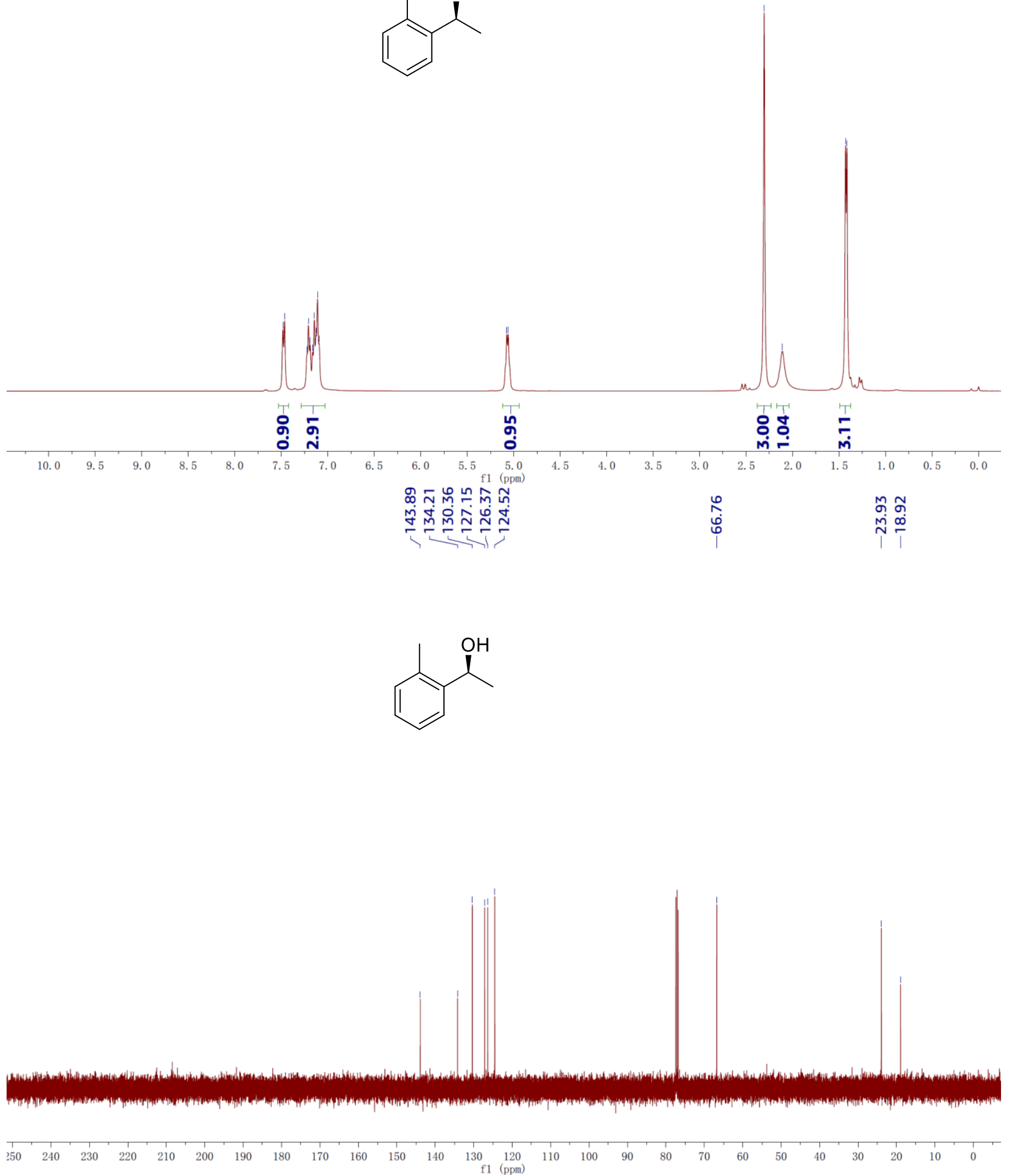
(S)-1-(2-methoxyphenyl)ethan-1-ol (2c)

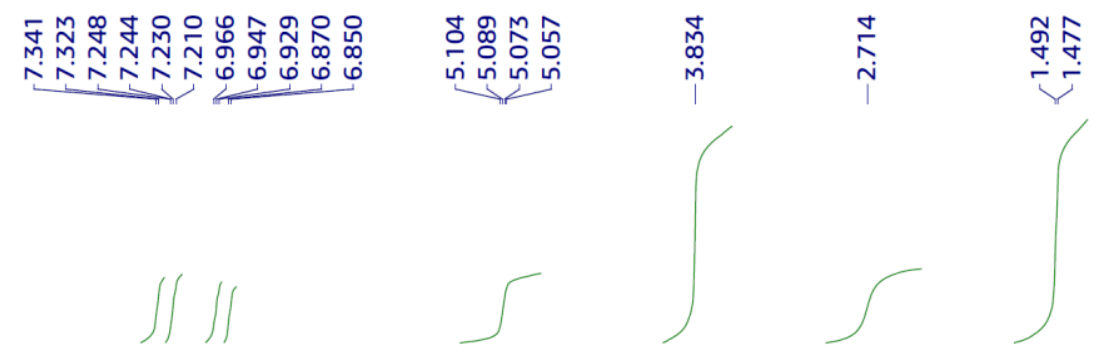<smiles>COc1ccccc1C(C)O</smiles>
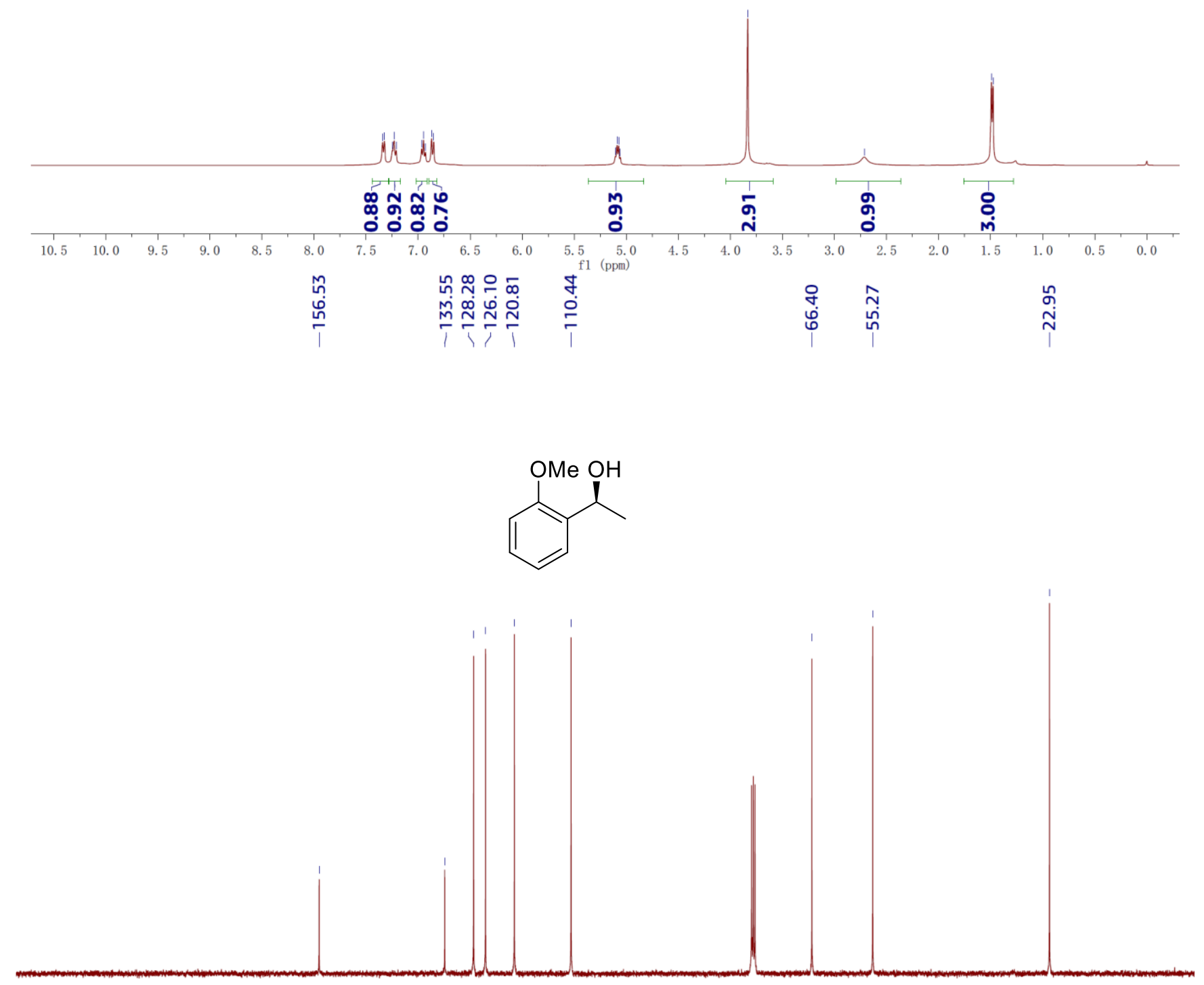
(S)-1-(2-bromophenyl)ethan-1-ol (2d)

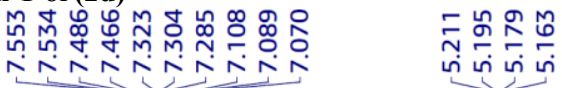

NָN

$\iint /$<smiles>C[C@@H](O)c1ccccc1Br</smiles>

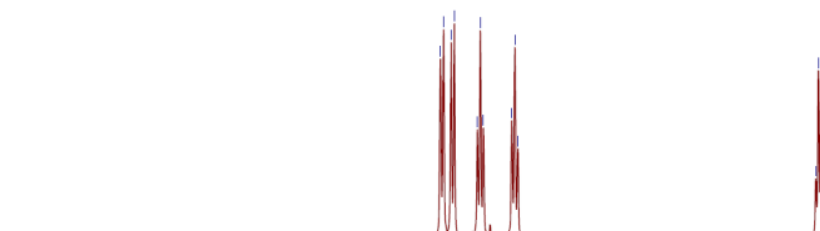

a.

$\begin{array}{llll}10.0 & 9.5 & 9.0 & 8.5\end{array}$<smiles>CC(O)c1ccccc1Br</smiles>

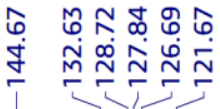

ตั่

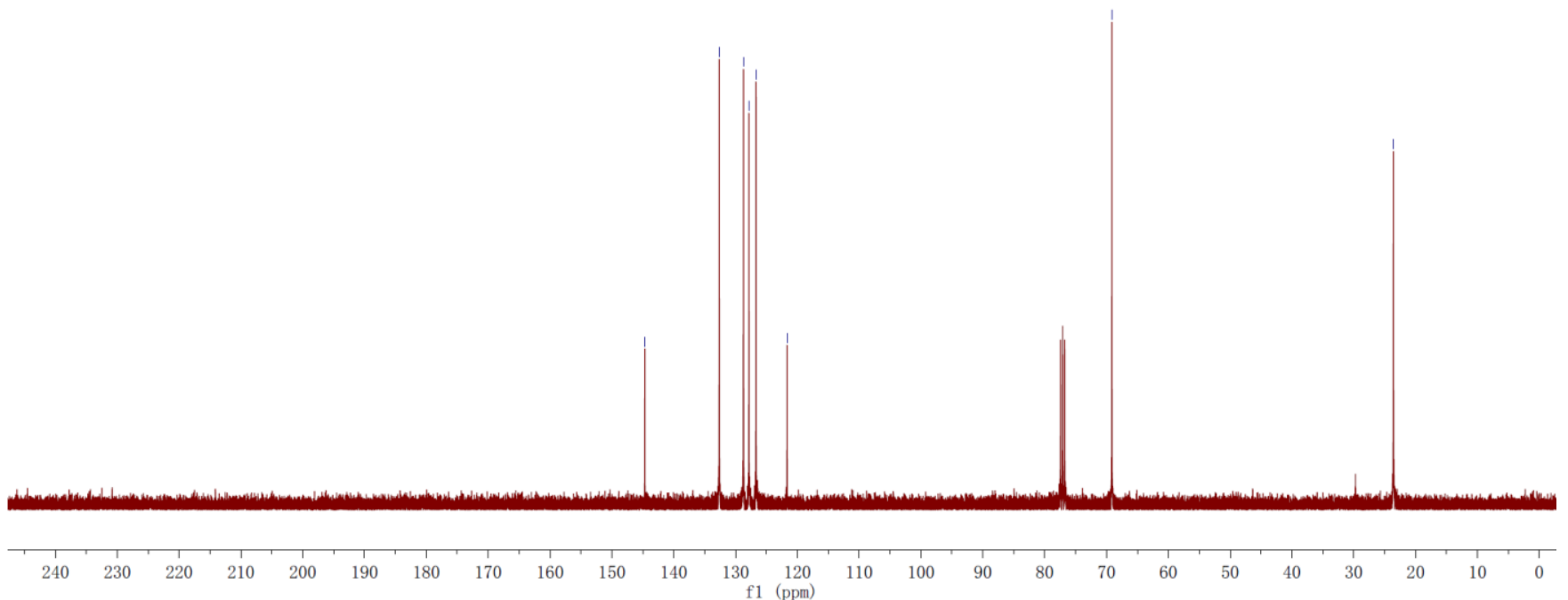


(S)-1-(4-bromophenyl)ethan-1-ol (2e)

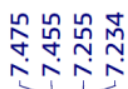

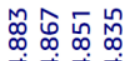

ナं

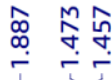
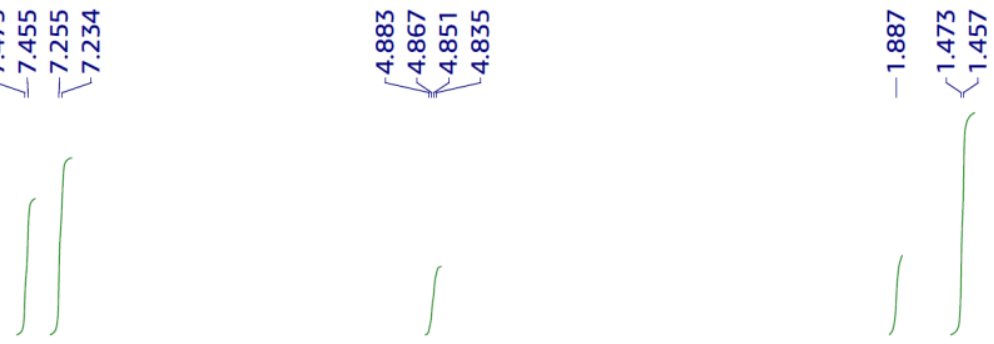<smiles>C[C@H](O)c1ccc(Br)cc1</smiles>
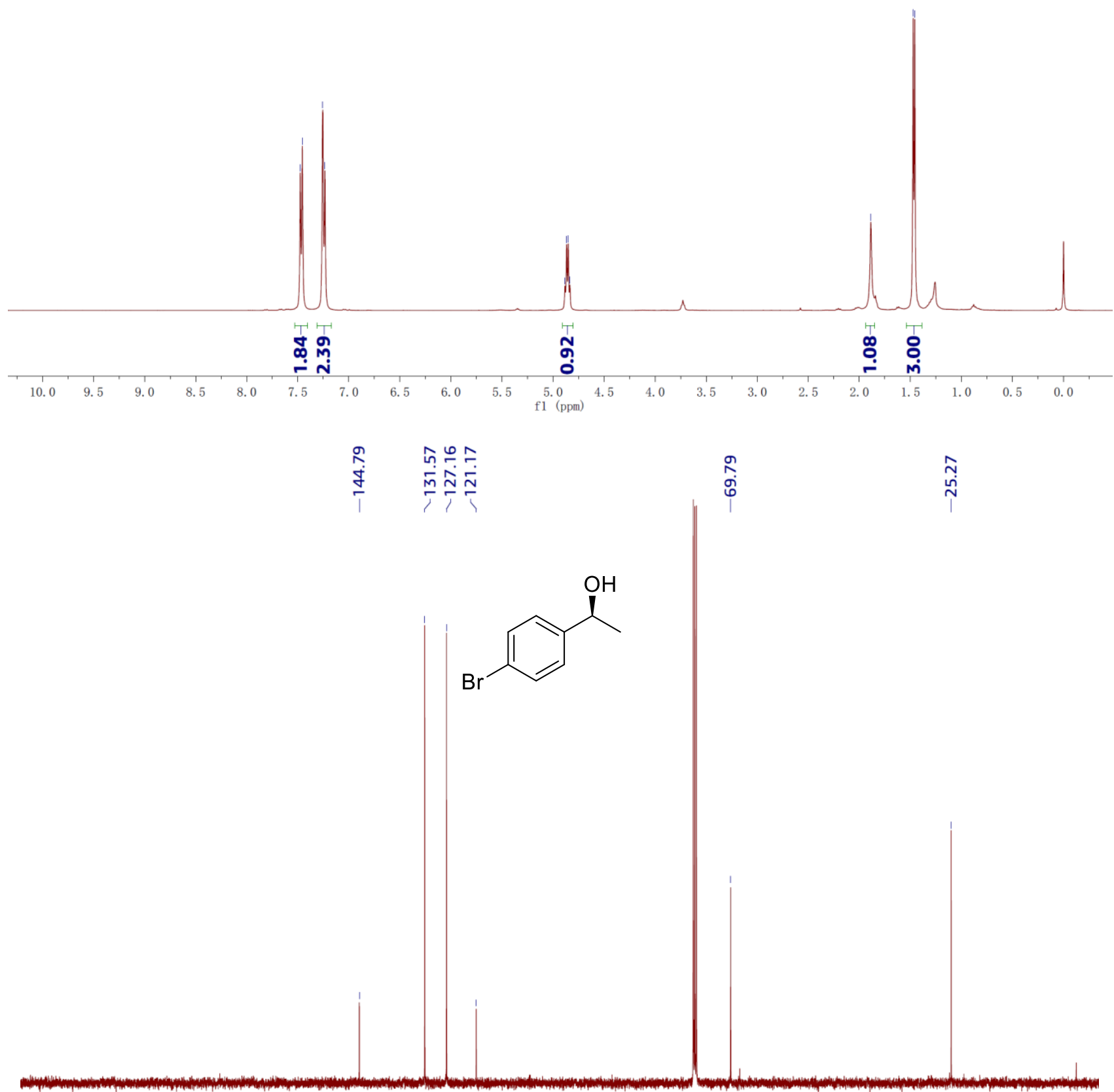

$\begin{array}{llllllllllll}210 & 200 & 190 & 180 & 170 & 160 & 150 & 140 & 130 & 120 & 110 & 100 \\ \mathrm{f} 1(\mathrm{ppm})\end{array}$ 
(S)-1-(4-iodophenyl)ethan-1-ol (2f)

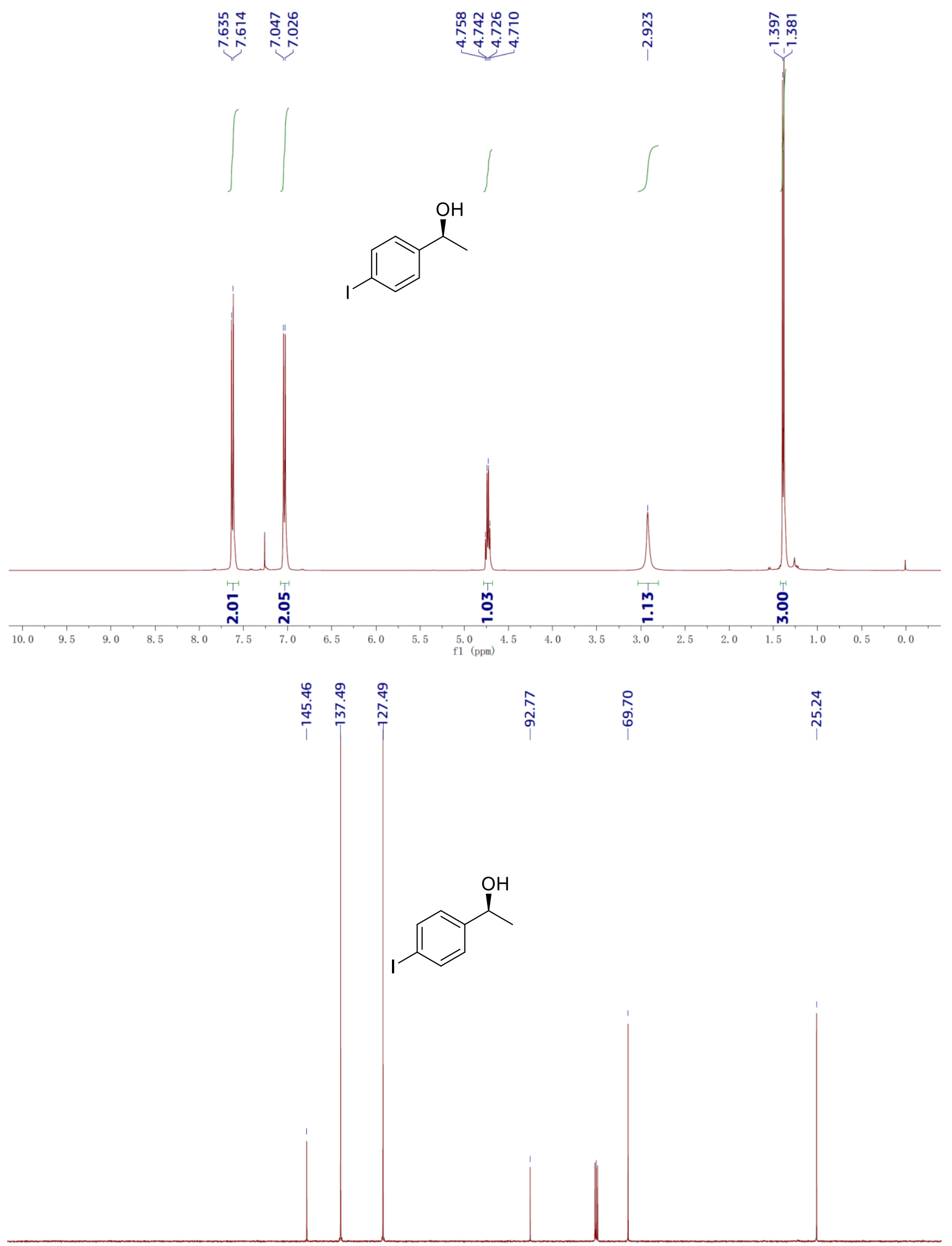

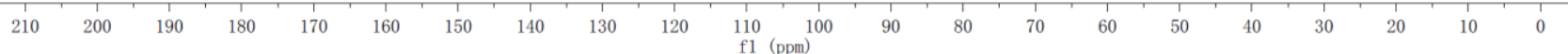


(S)-1-(2-chlorophenyl)ethan-1-ol (2g)

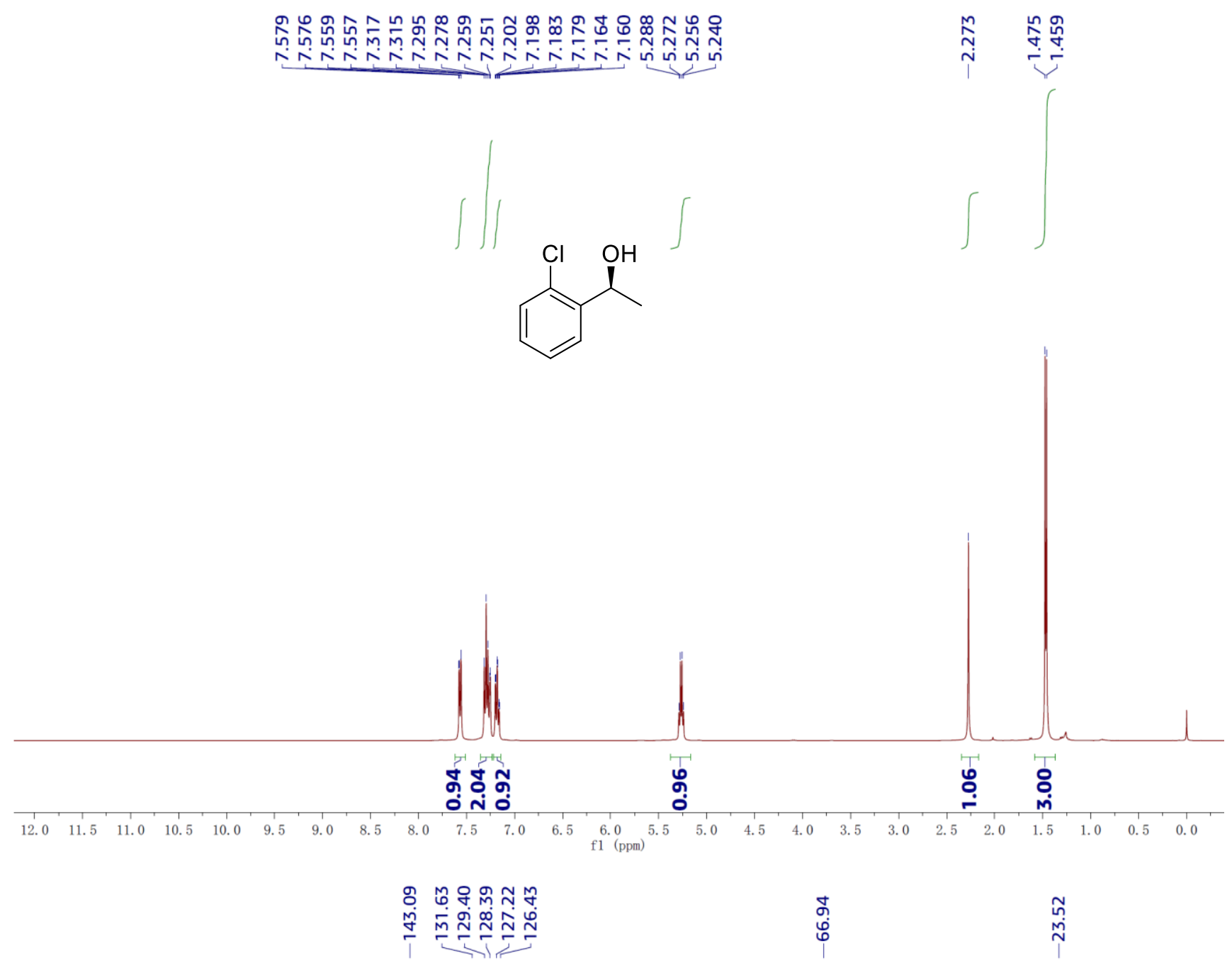<smiles>C[C@H](O)c1ccccc1Cl</smiles>

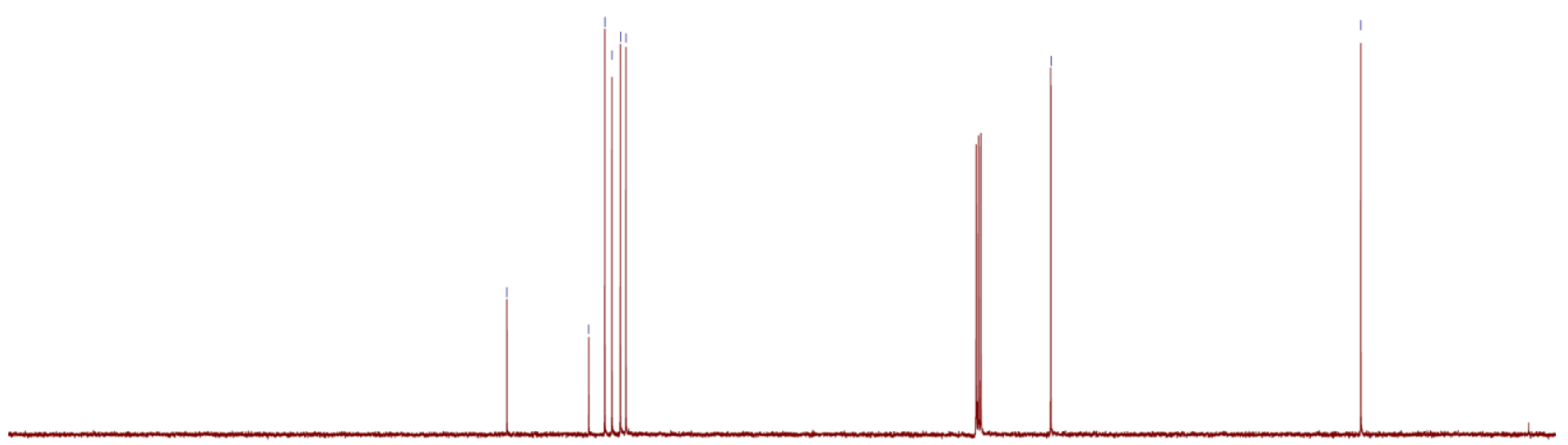

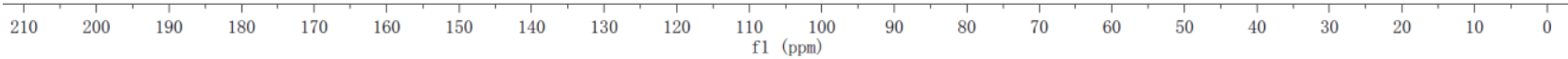


(S)-1-(2-fluorophenyl)ethan-1-ol (2h)

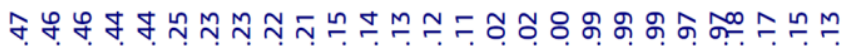

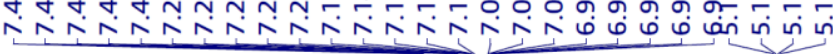

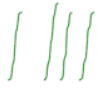<smiles>C[C@@H](O)c1ccccc1F</smiles>

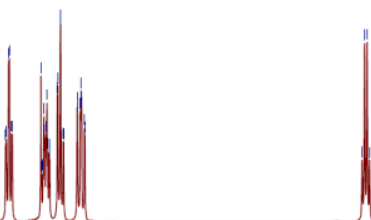

สีร์ำำ

क

읃

웅

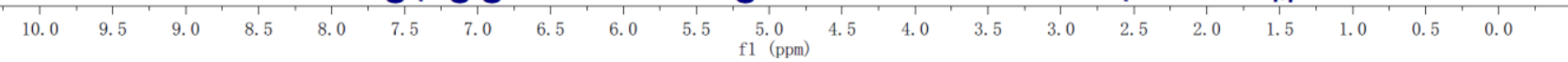

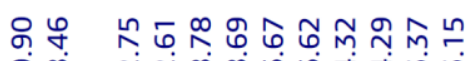

它空

₹ $\bar{m}$

它

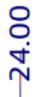

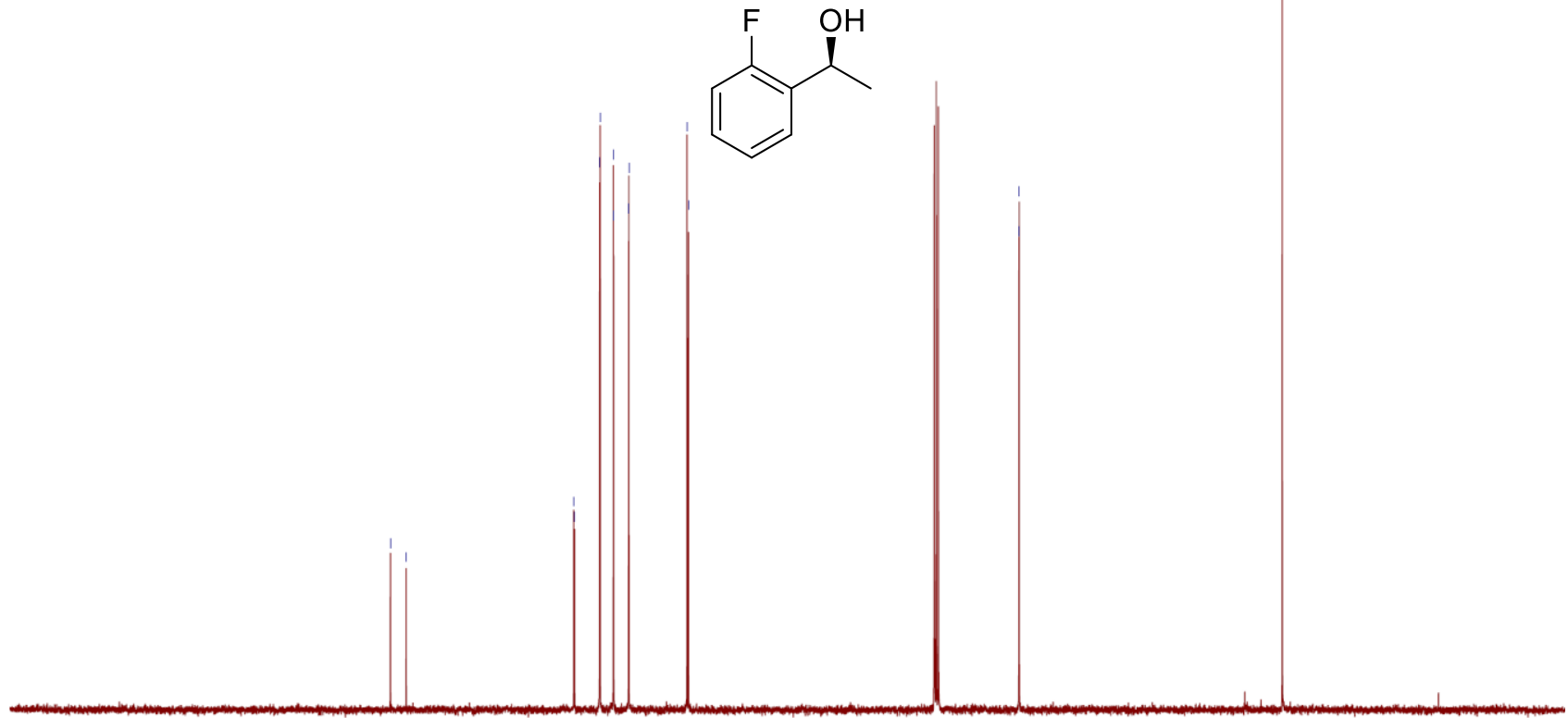

$\begin{array}{llllllllllll}210 & 200 & 190 & 180 & 170 & 160 & 150 & 140 & 130 & 120 & 110 & \begin{array}{c}100 \\ \mathrm{f} 1(\mathrm{ppm})\end{array}\end{array}$ 
<smiles>C[C@H](O)c1ccccc1F</smiles>

\begin{tabular}{rlllllllllllllllllllllll}
\hline & 10 & 0 & -10 & -20 & -30 & -40 & -50 & -60 & -70 & -80 & -90 & -100 & -110 & -120 & -130 & -140 & -150 & -160 & -170 & -180 & -190 & -200
\end{tabular} 
(S)-1-(4-fluorophenyl)ethan-1-ol (2i)

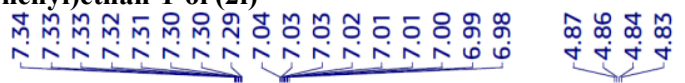

COH

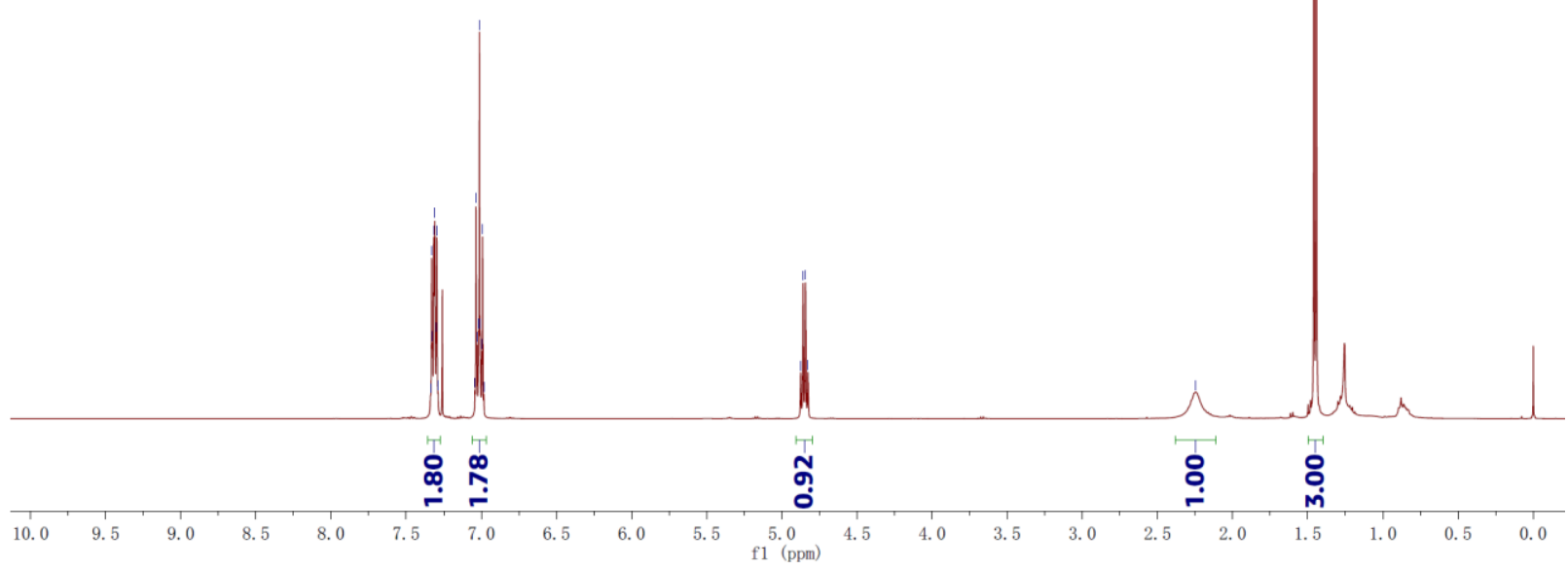



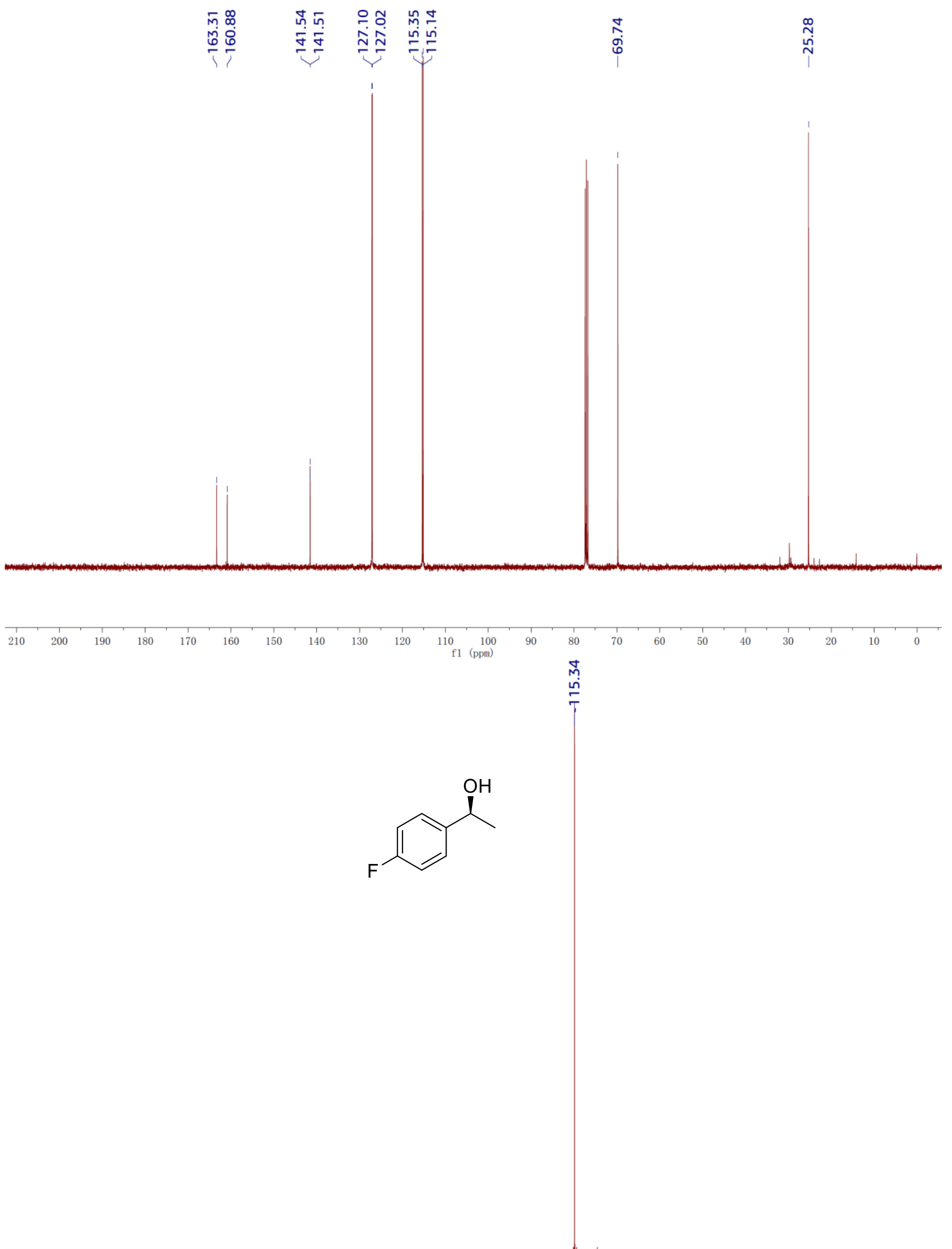

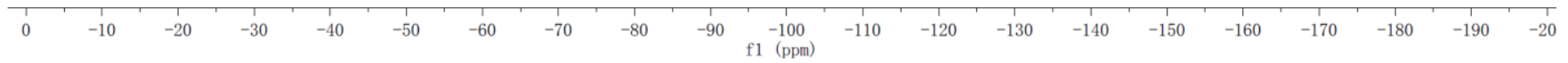


(S)-1-(4-(trifluoromethyl)phenyl)ethan-1-ol (2j)
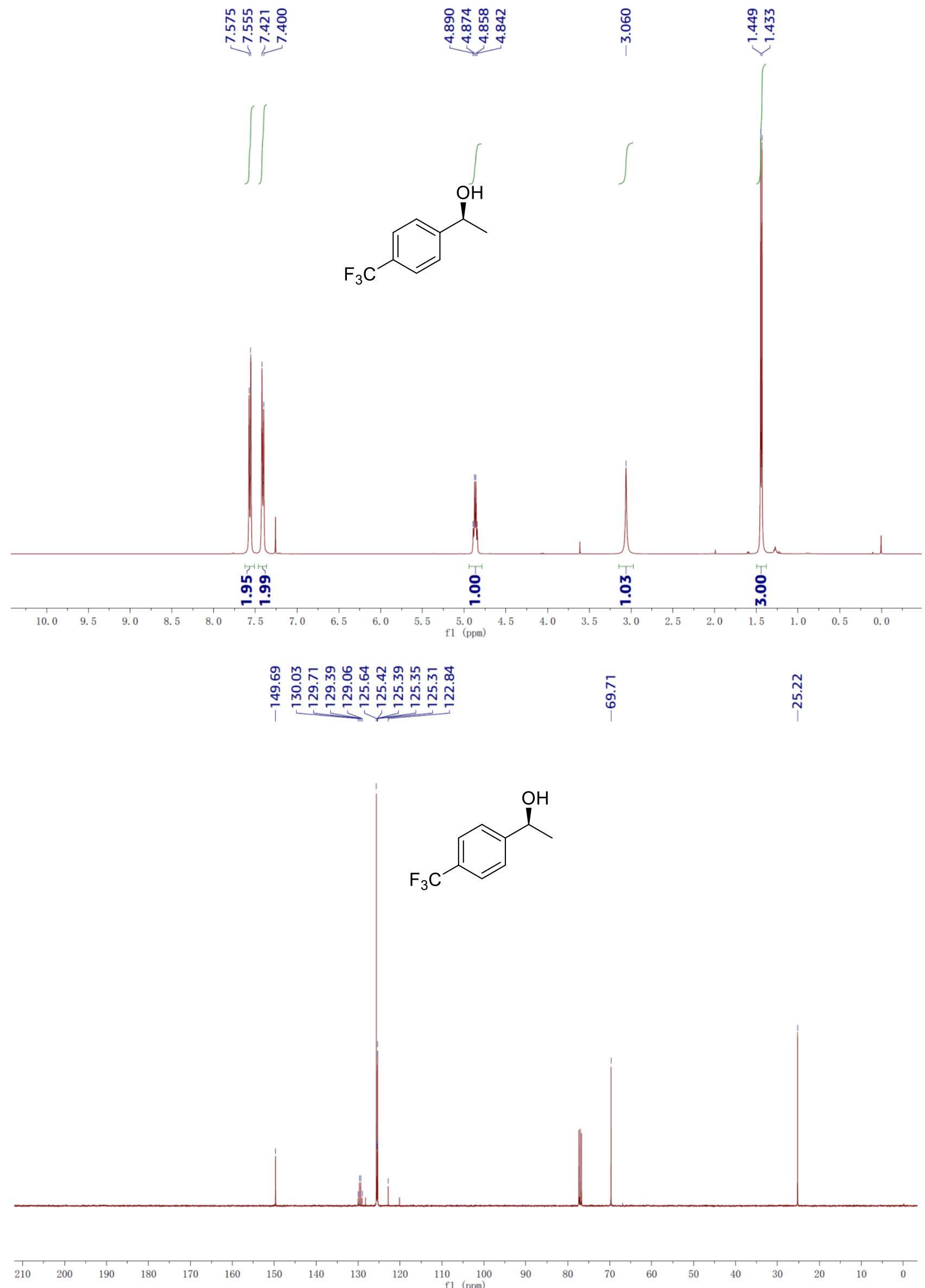
<smiles>C[C@H](O)c1ccc(C(F)(F)F)cc1</smiles>

\begin{tabular}{llllllllllllllllllllllllll}
\hline 110 & 90 & 80 & 70 & 60 & 50 & 40 & 30 & 20 & 10 & 0 & -10 & -30 & -50 & -70 & -90 & -110 & -130 & -150 & -170 & -190 & -210 & -230 & -250 & -1 &
\end{tabular} 
(S)-1-(2-(trifluoromethyl)phenyl)ethan-1-ol (2k)
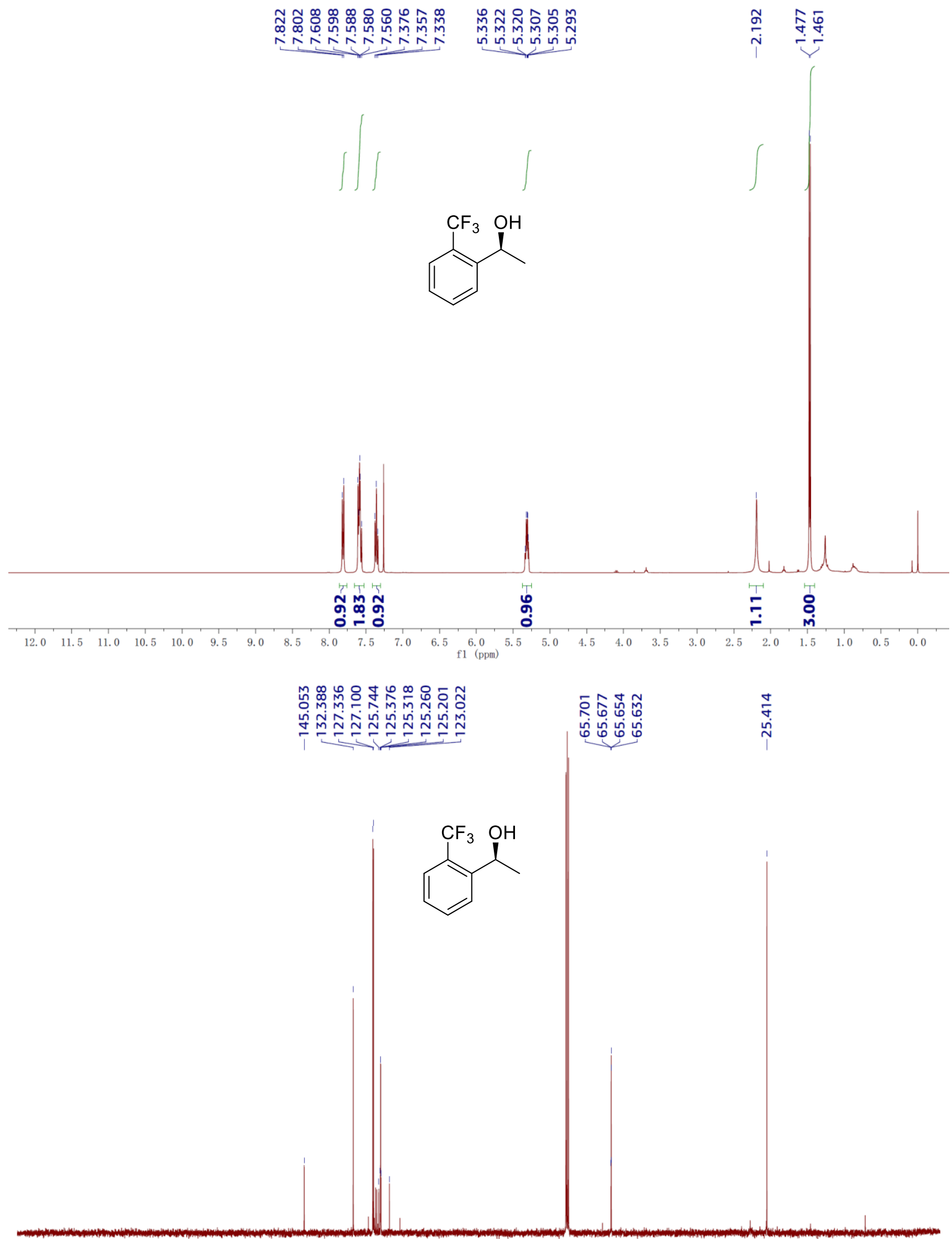

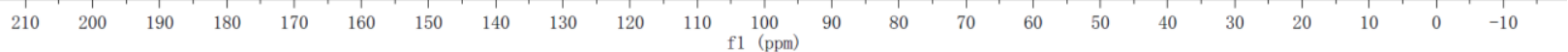



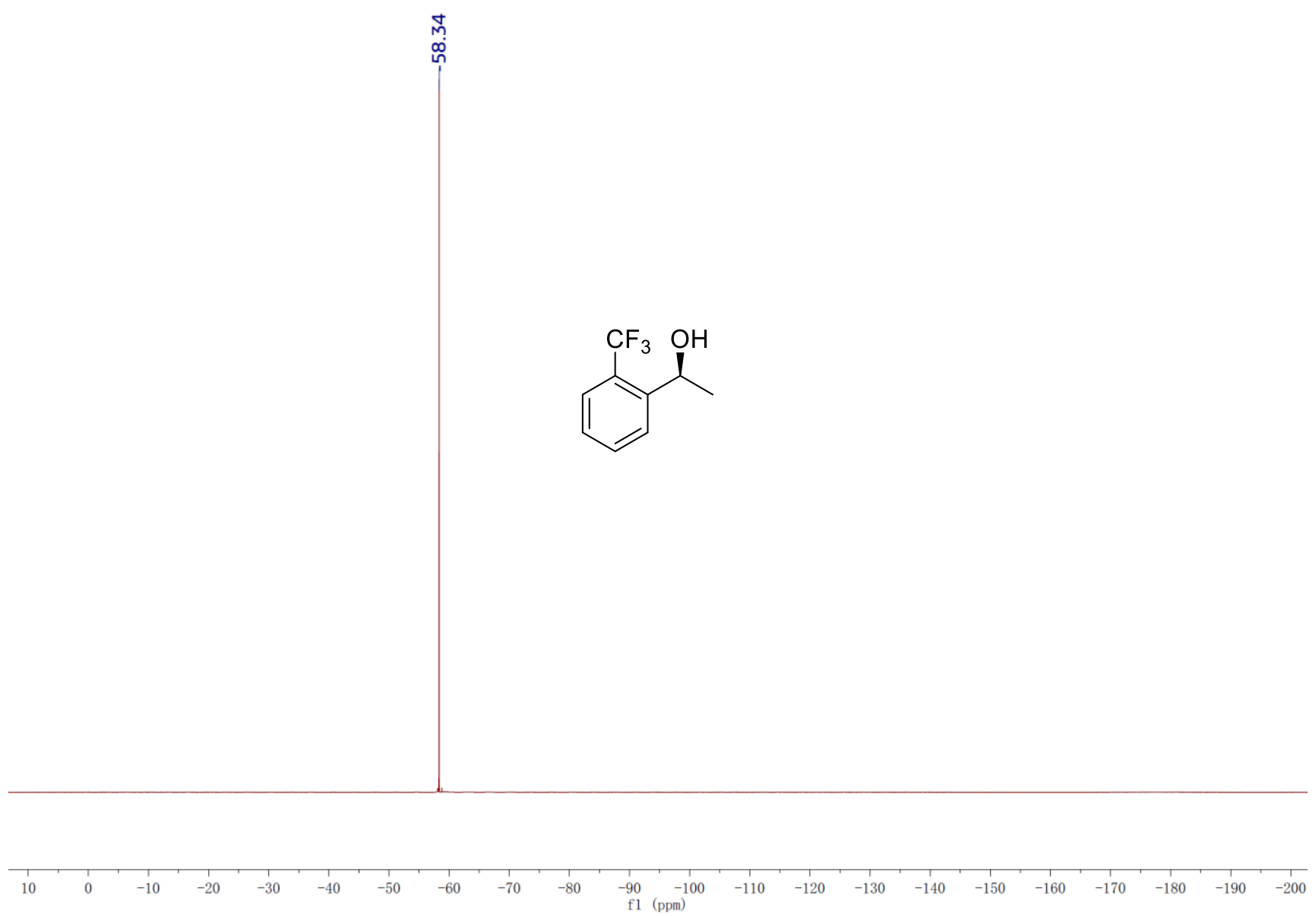

S60 
(S)-4-(1-hydroxyethyl)benzonitrile (2I)

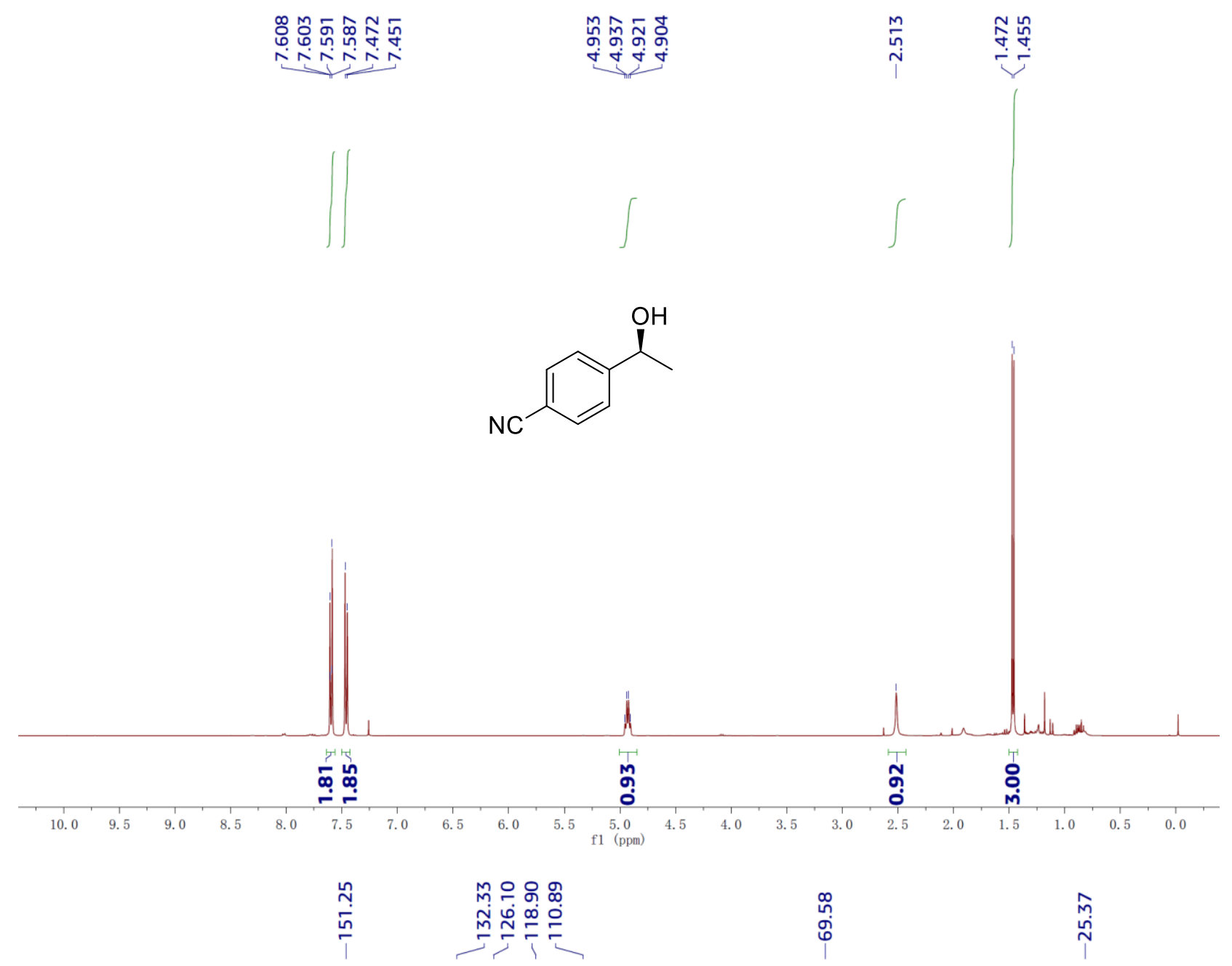

(O)

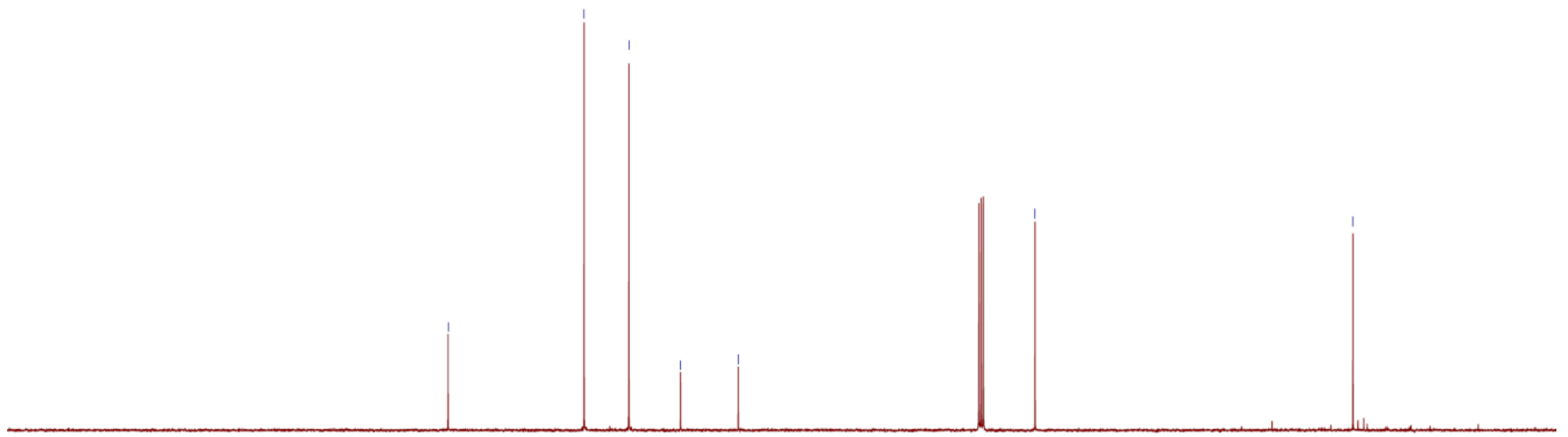


(S)-1-(2,6-dichloro-3-fluorophenyl)ethan-1-ol (2m)

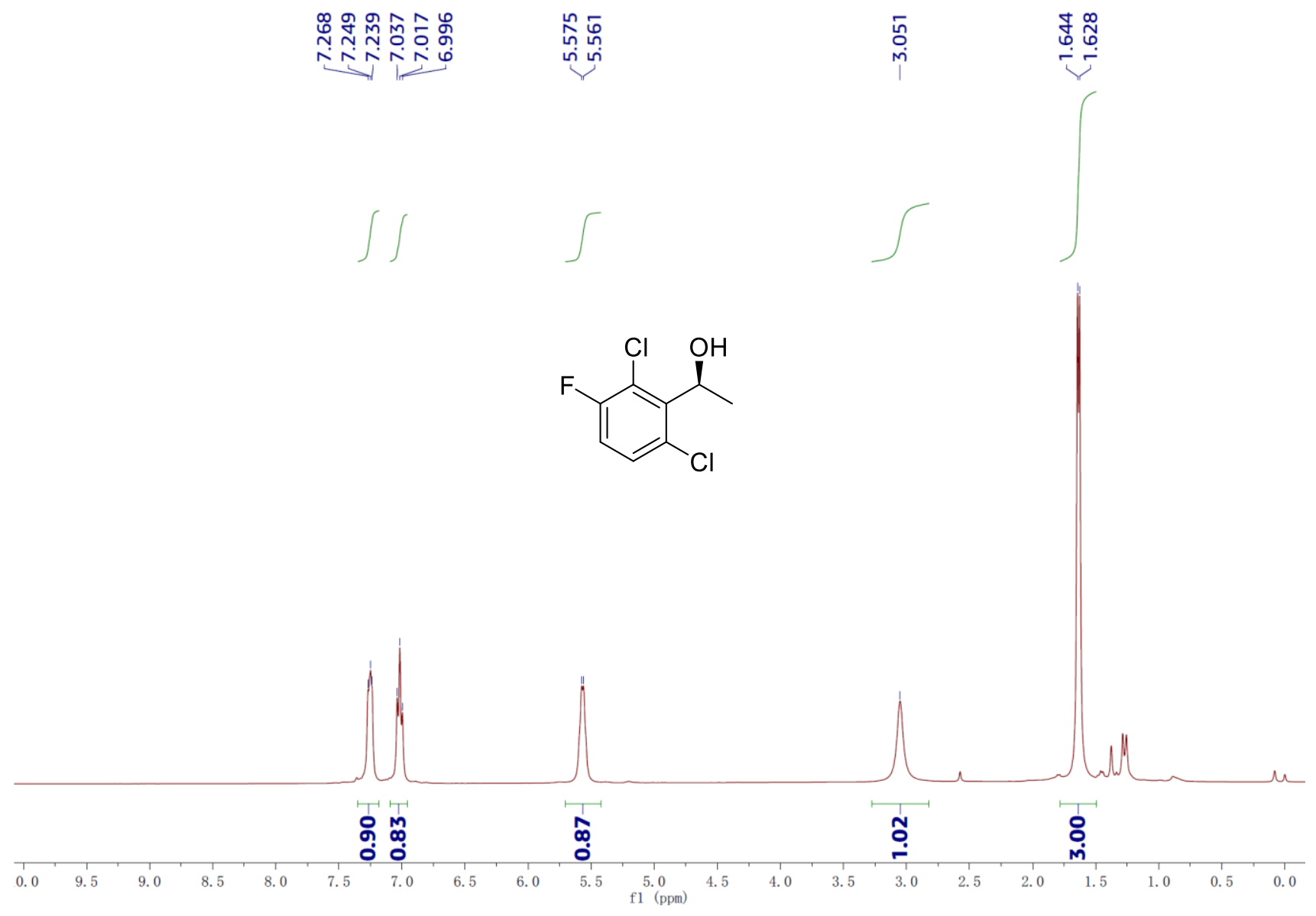

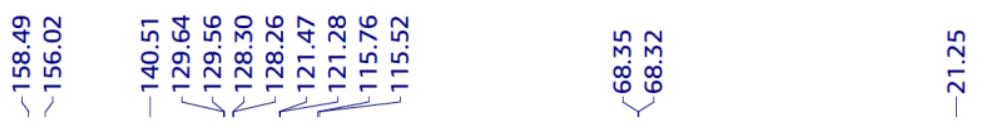

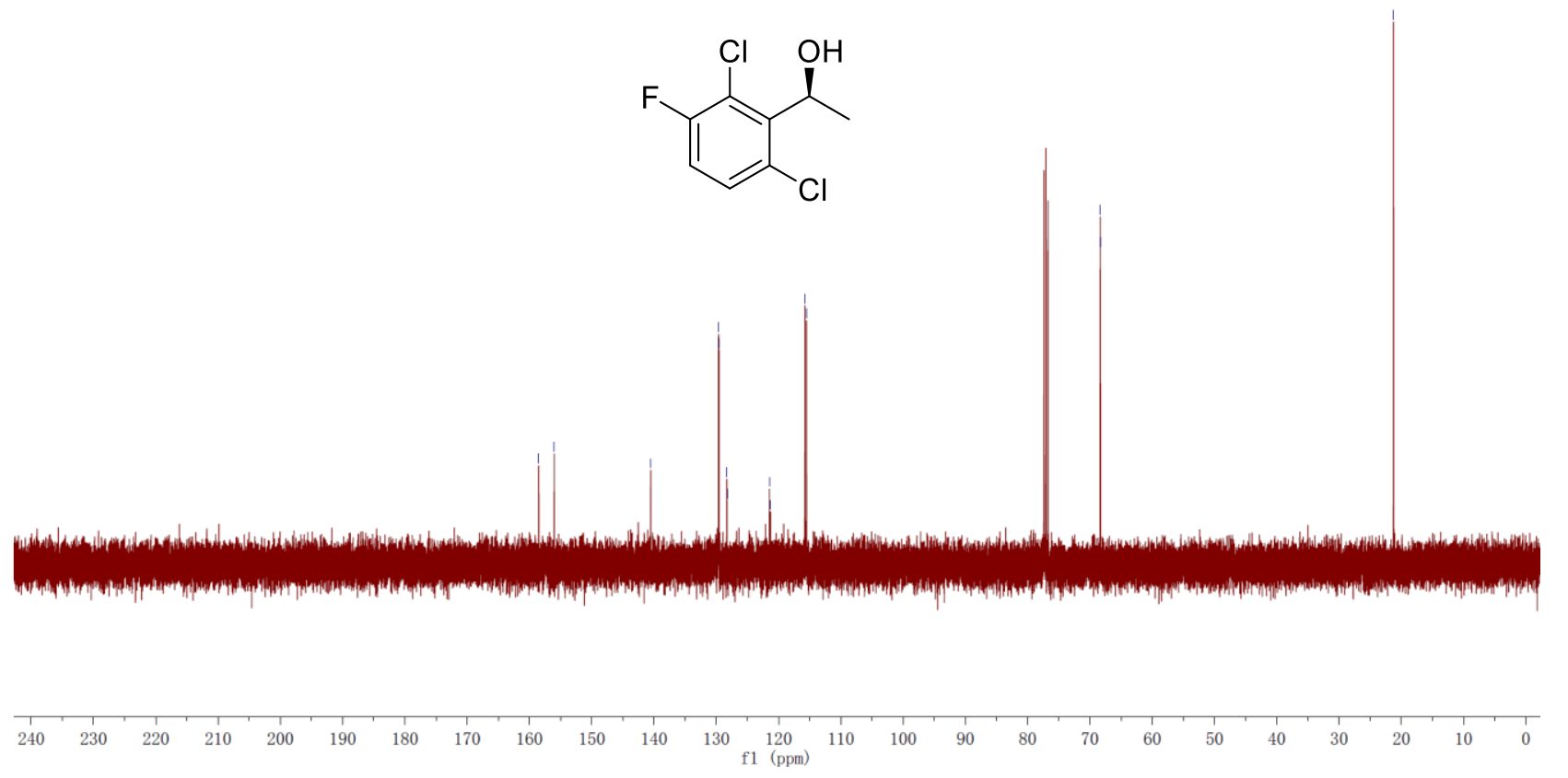



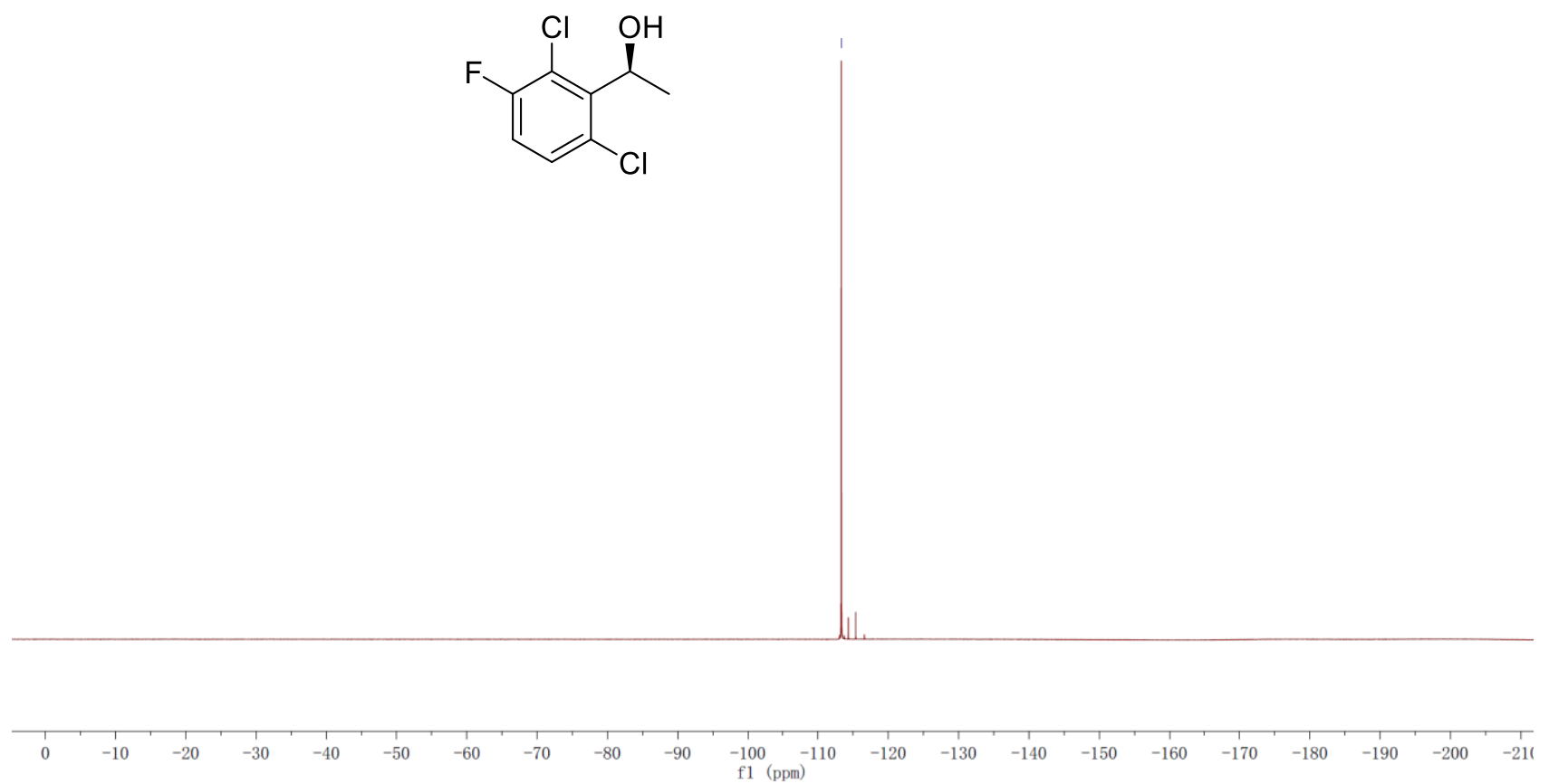
(S)-1-(2,6-dichlorophenyl)ethan-1-ol (2n)
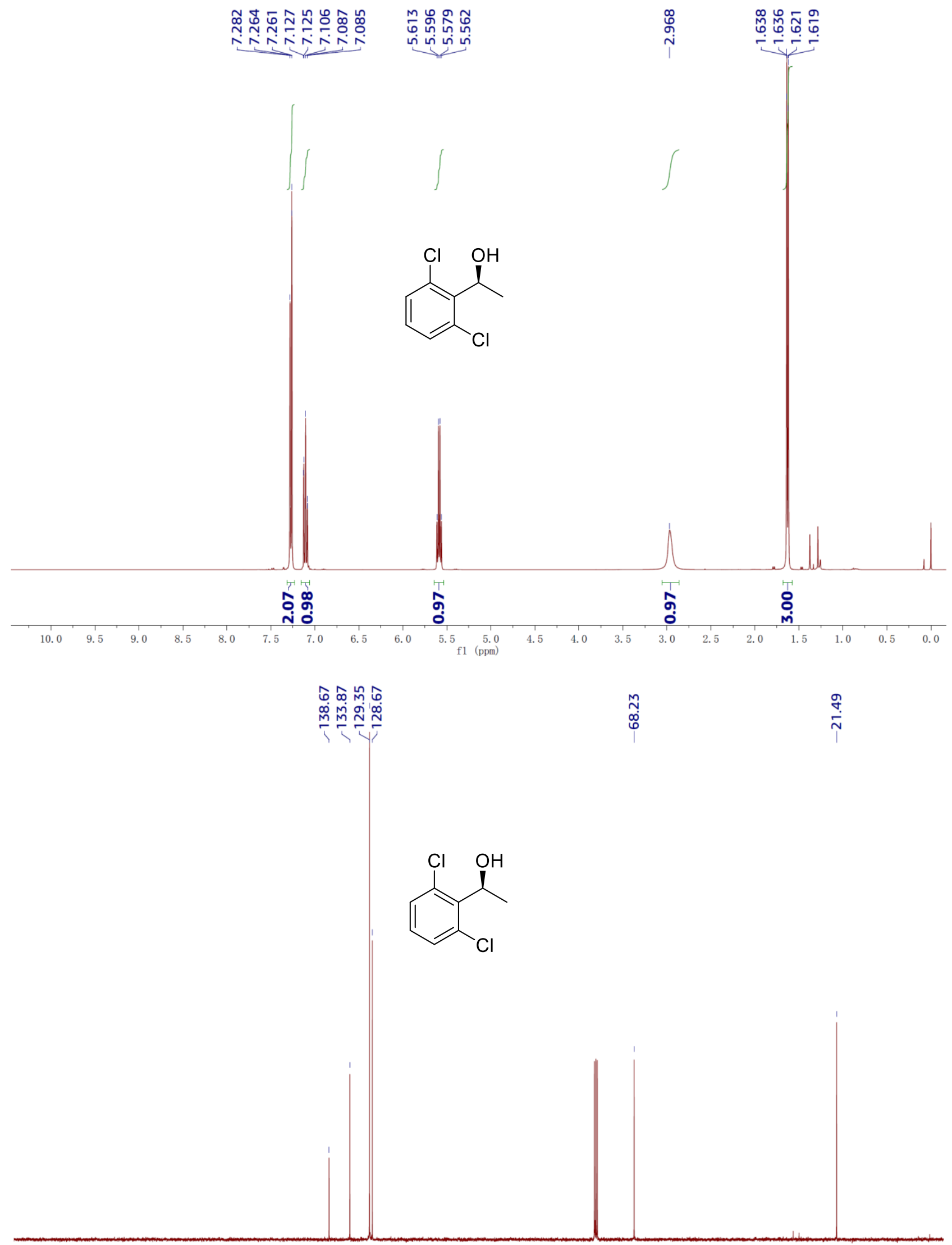

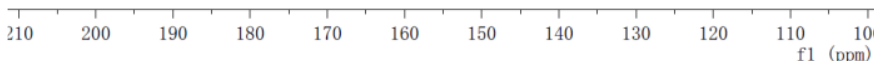


(S)-1-(2,6-dimethylphenyl)ethan-1-ol (2o)

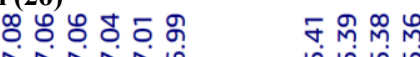

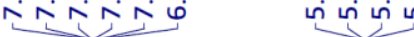

๒

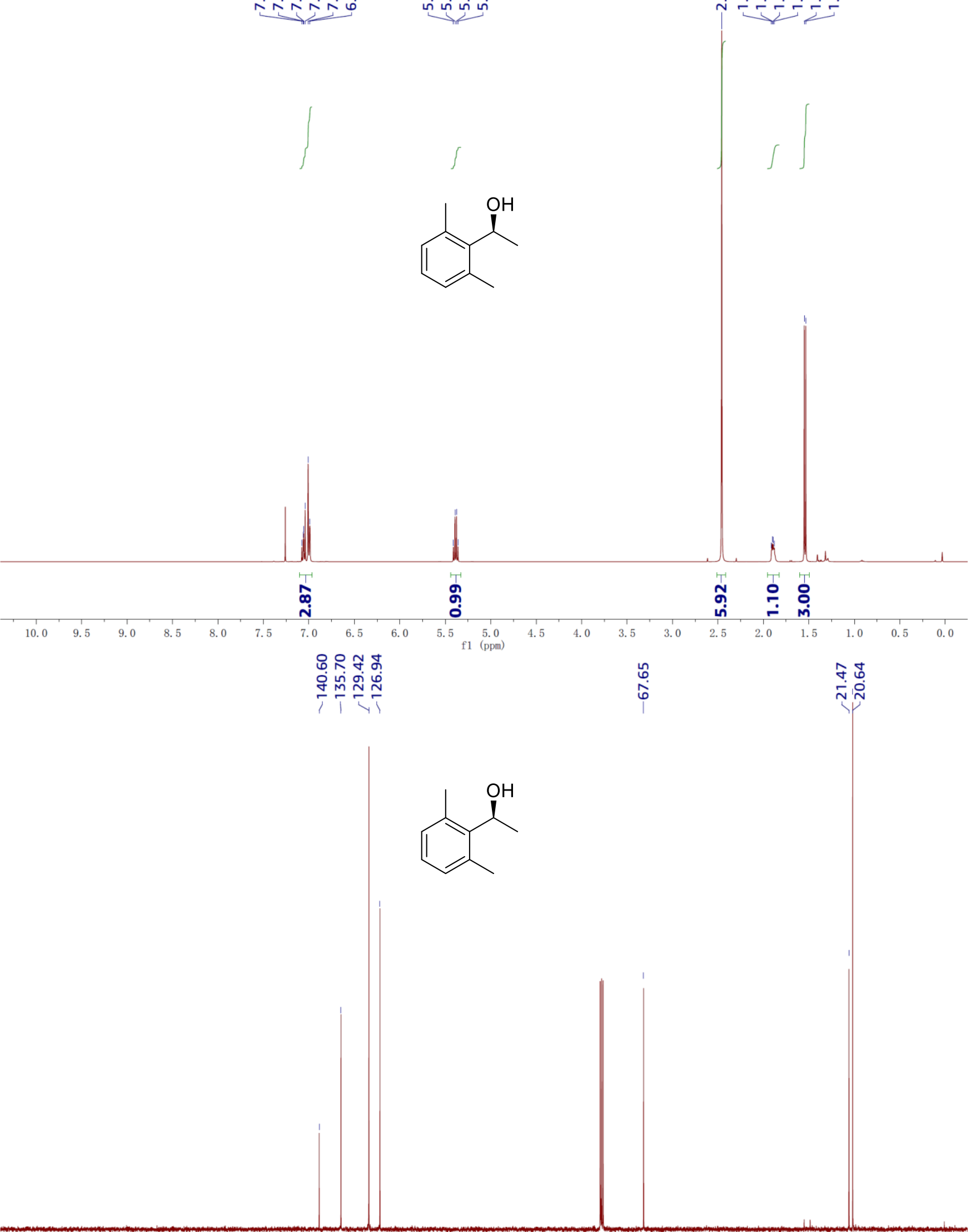

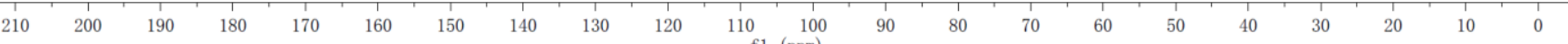


(S)-1-(furan-2-yl)ethan-1-ol (2p)
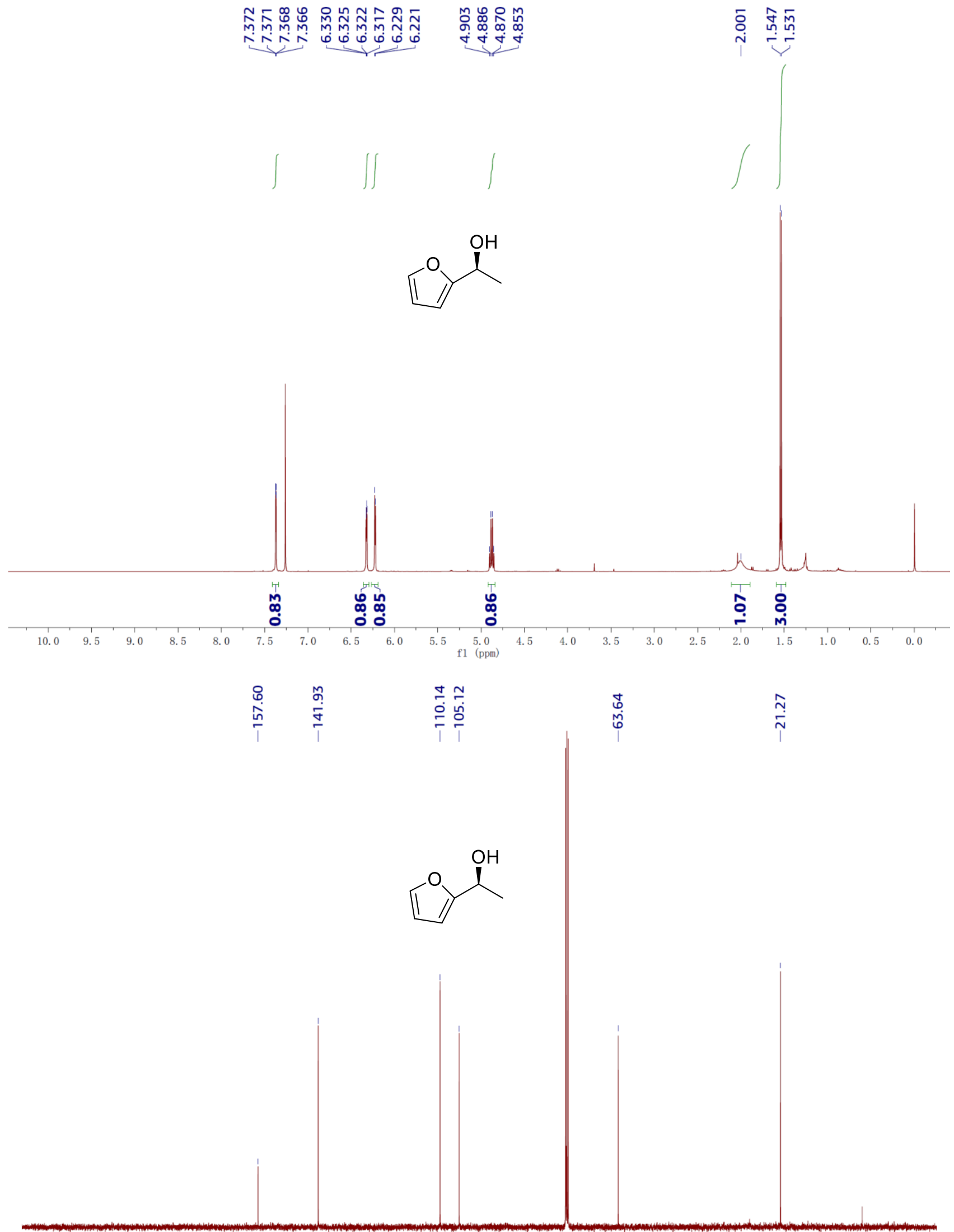
(S)-1-(thiophen-2-yl)ethan-1-ol (2q)

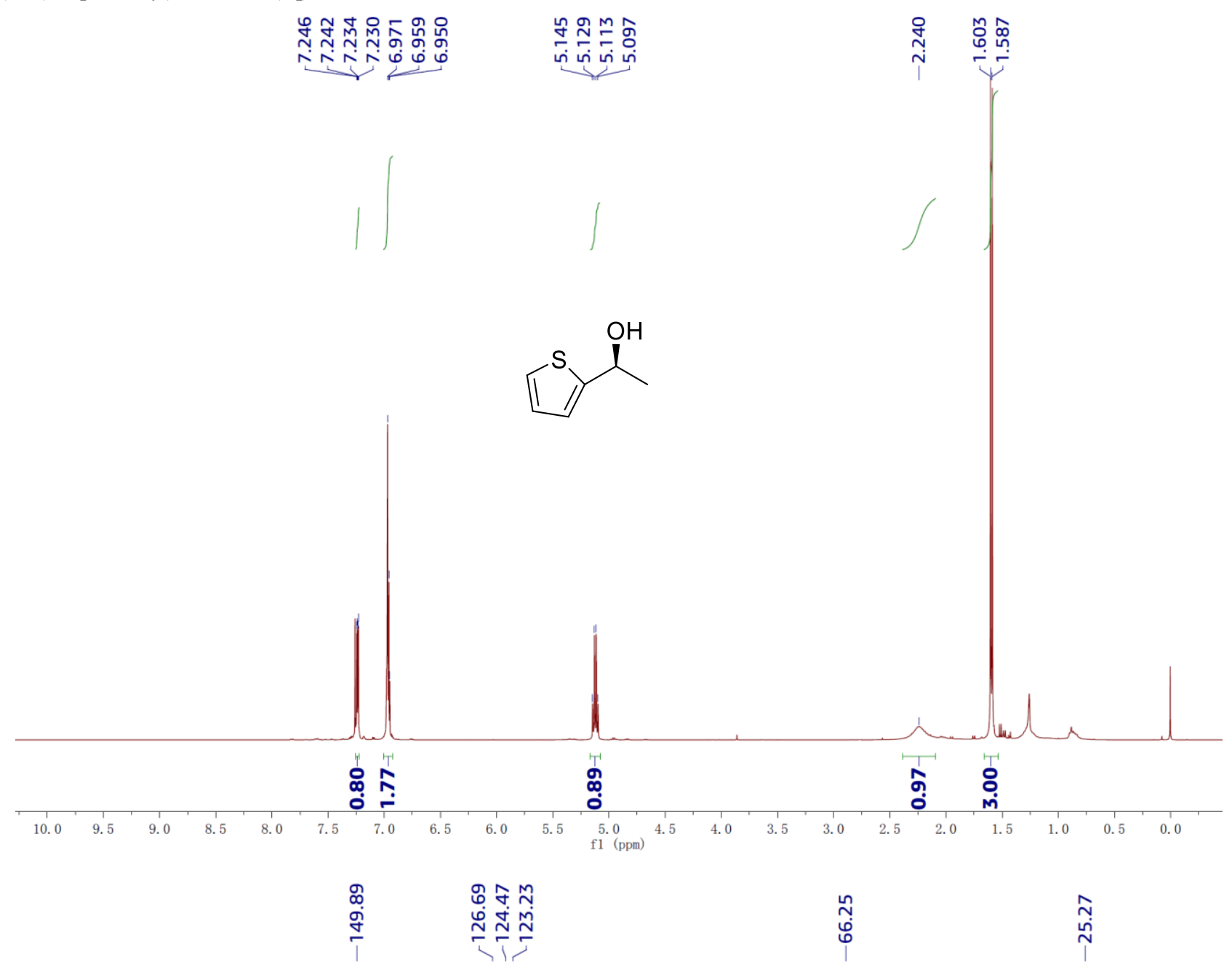

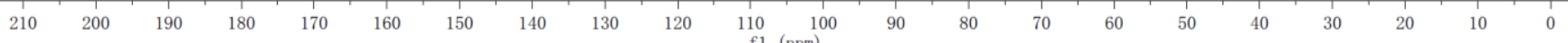


(S)-2,2-dimethyl-1-phenylpropan-1-ol (4a)

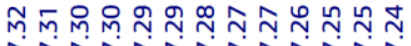

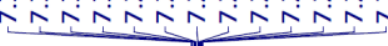
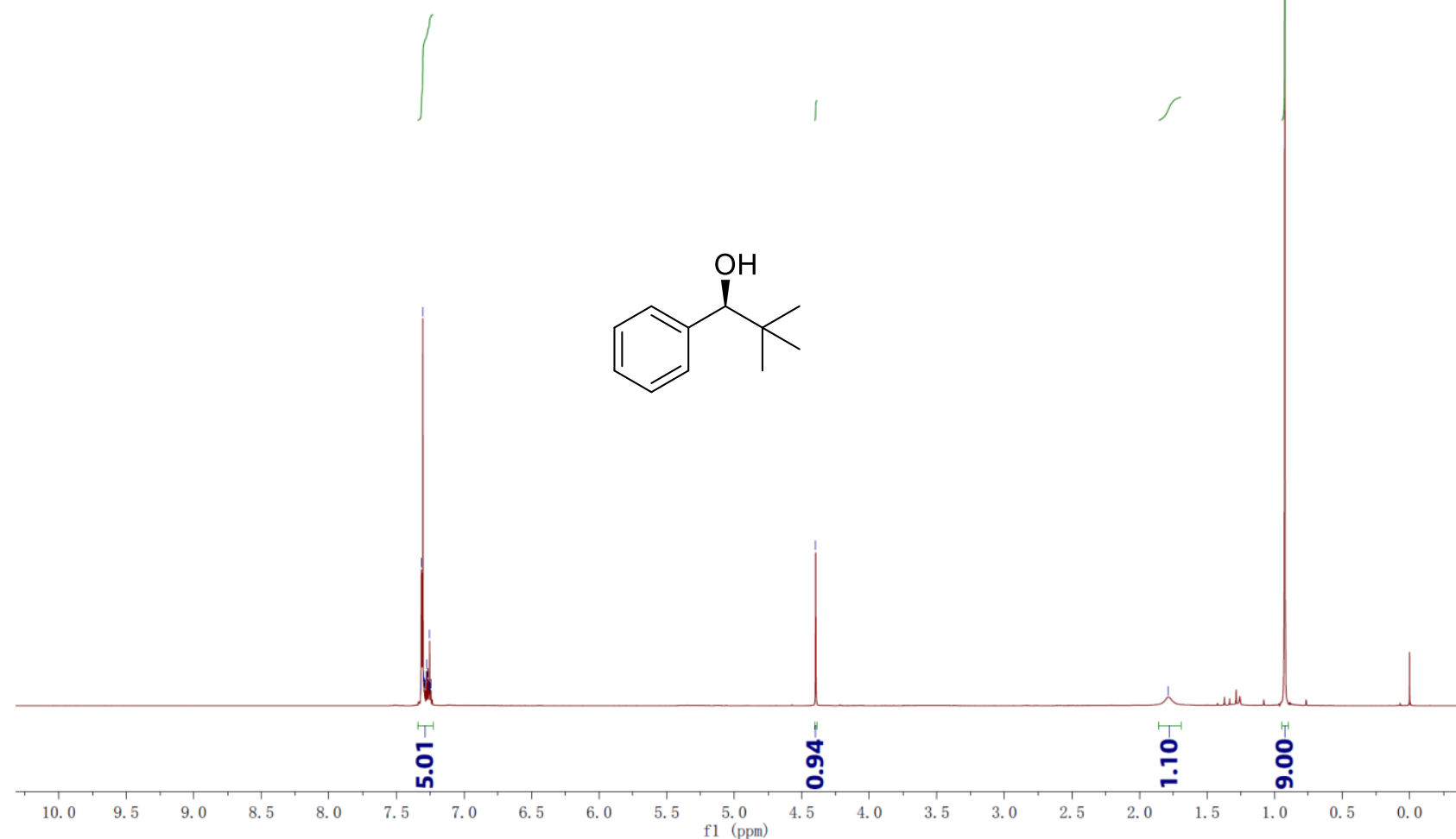

ำ
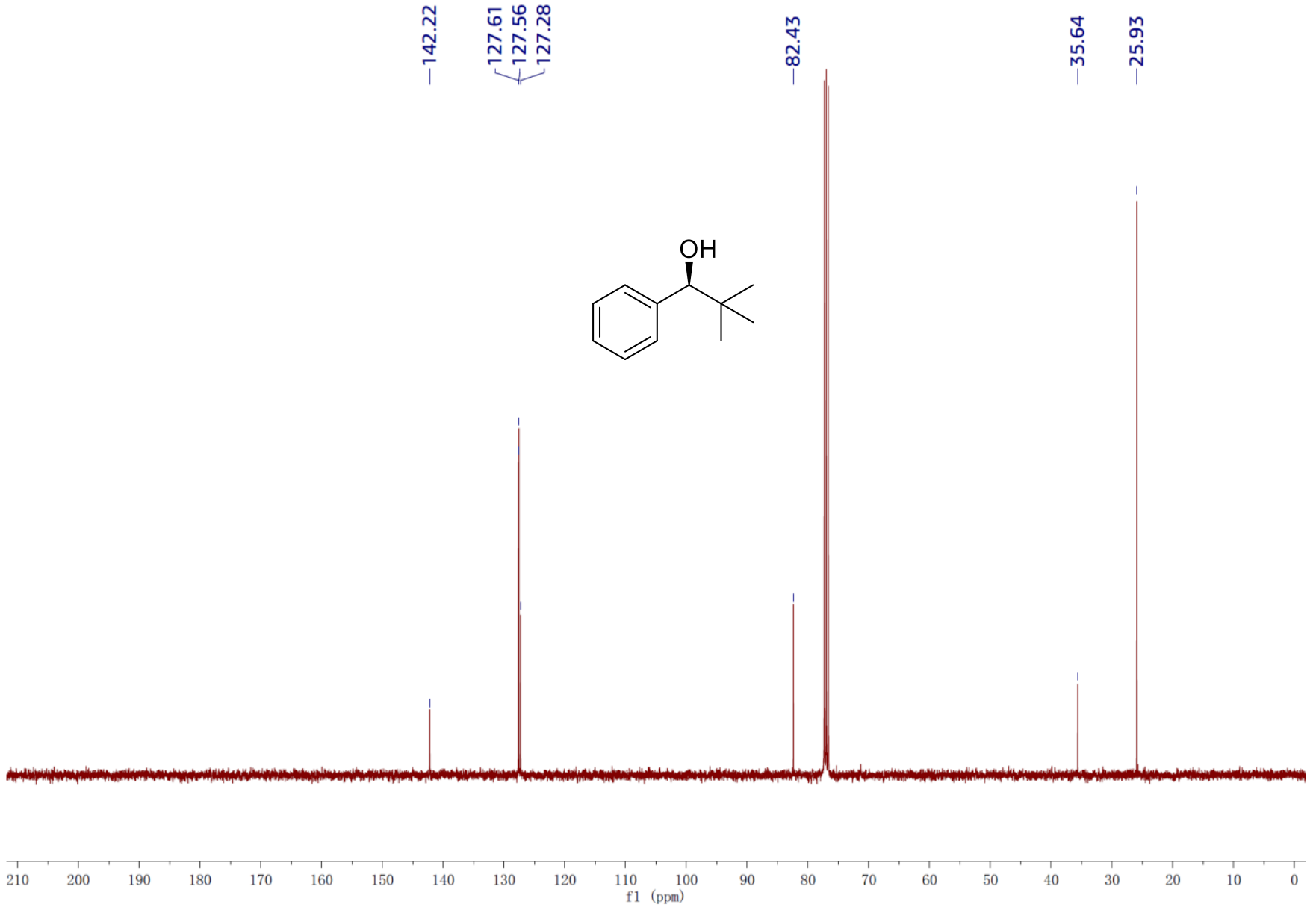


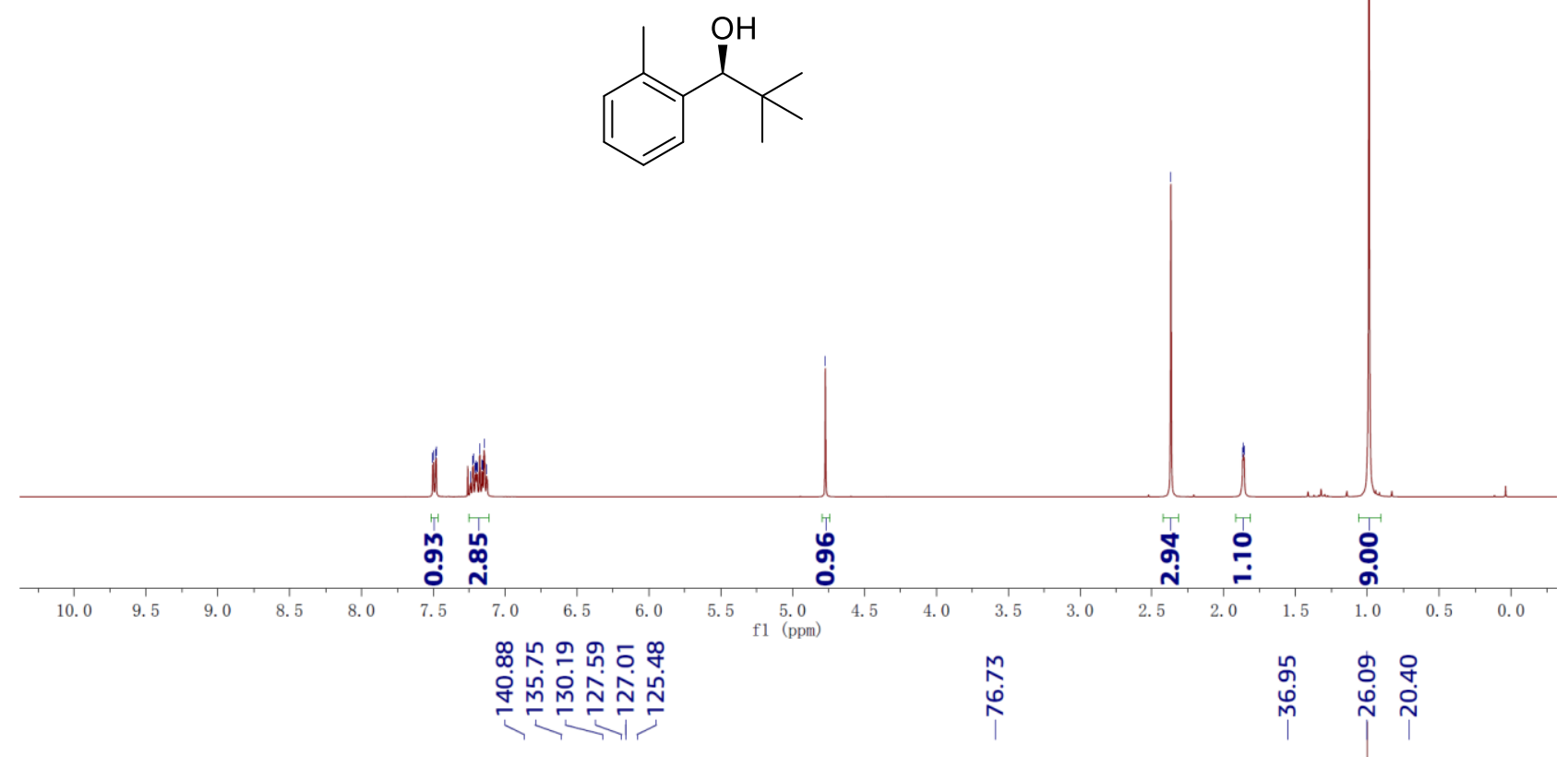<smiles>Cc1ccccc1[C@@H](O)C(C)(C)C</smiles>

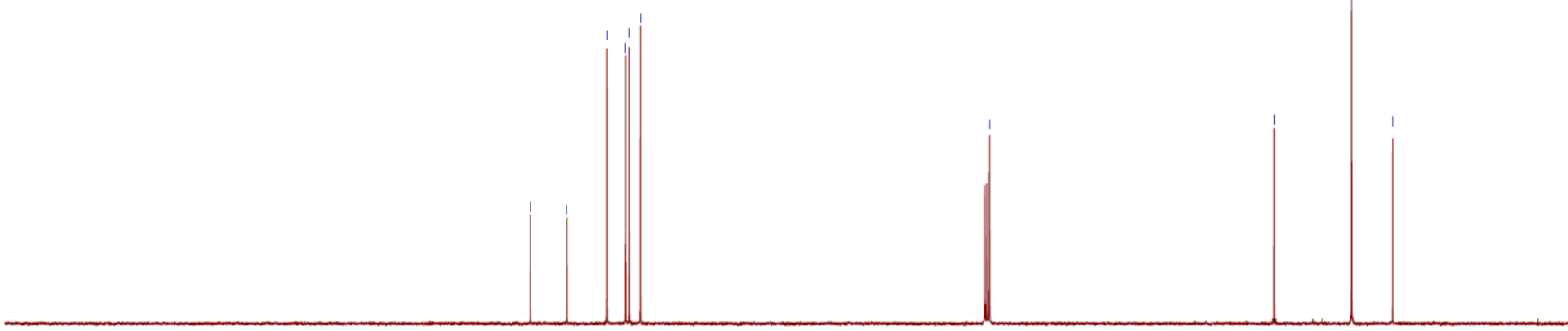

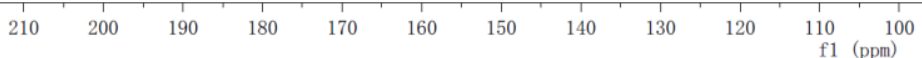


(S)-1-(4-methoxyphenyl)-2,2-dimethylpropan-1-ol (4c)

$$
\text { กูก กุ๊ }
$$

نำ

$\stackrel{\substack{n \\ i}}{\stackrel{n}{1}}$

$\stackrel{\text { Ò }}{i}$

ஜே
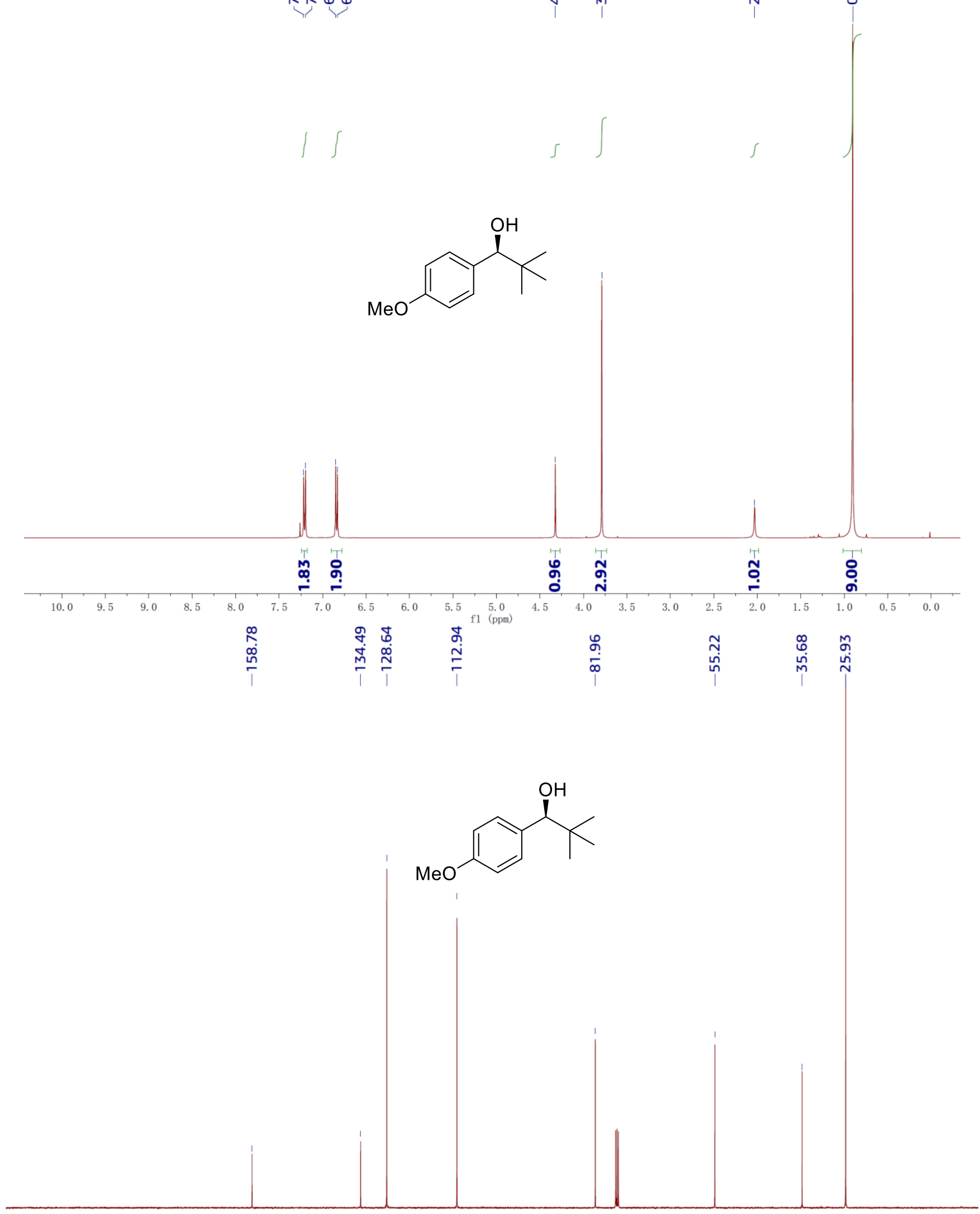

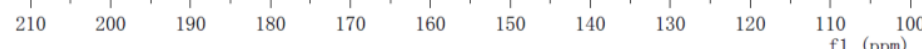


(S)-1-(2-bromophenyl)-2,2-dimethylpropan-1-ol (4d)

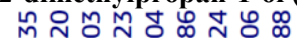

กำ

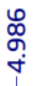

$\stackrel{\leftrightarrow}{\text { i }}$

ڤ్m

(1)
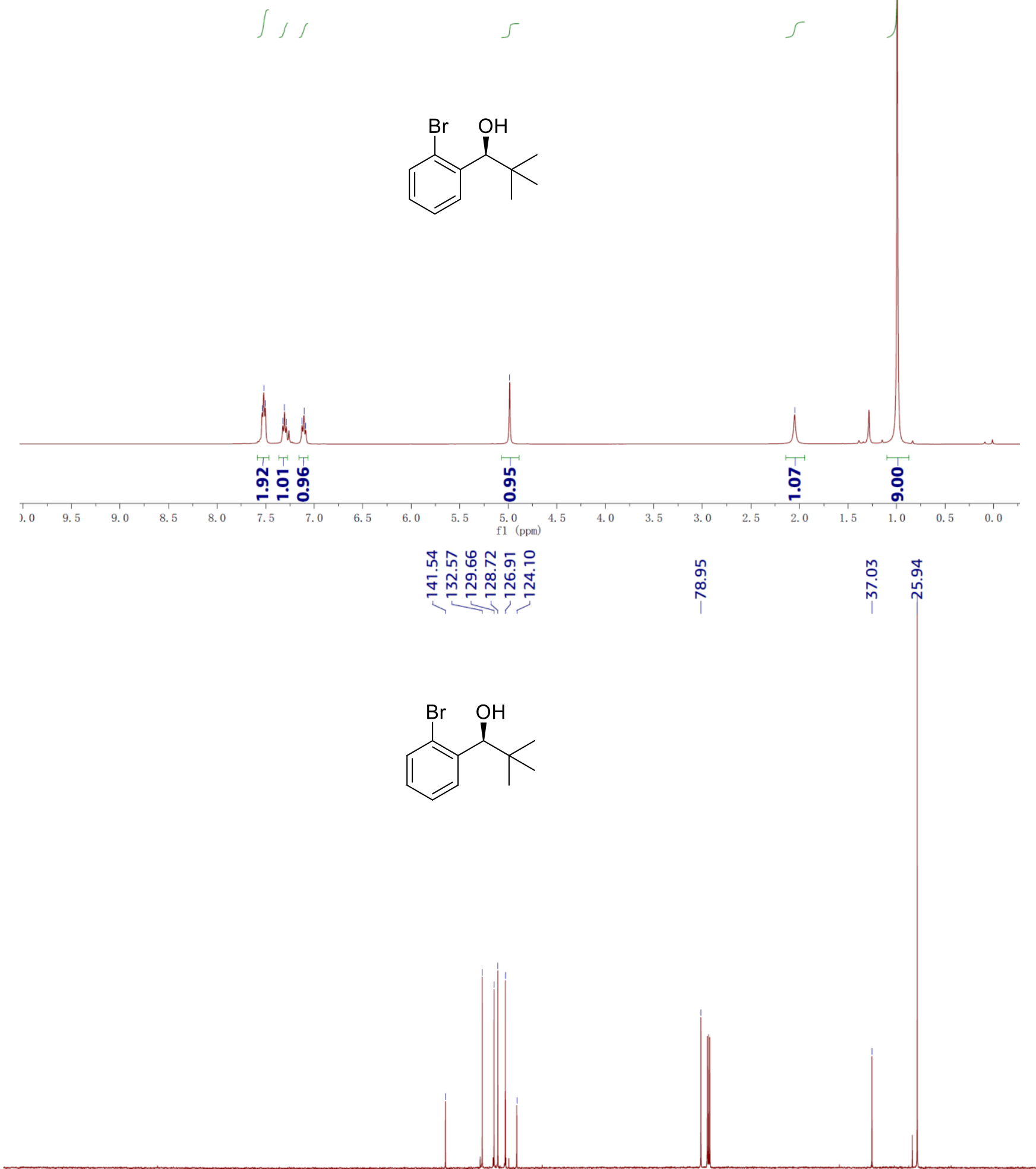

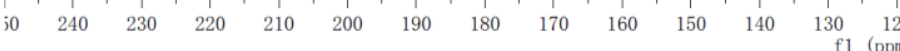


(S)-1-(3-bromophenyl)-2,2-dimethylpropan-1-ol (4e)

ํำ ำ

ヘ

$\stackrel{\substack{n \\ i}}{\stackrel{n}{i}}$

$\stackrel{\text { Mo }}{\text { i }}$

ஃீ.

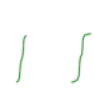

$\mathrm{C}_{1}$
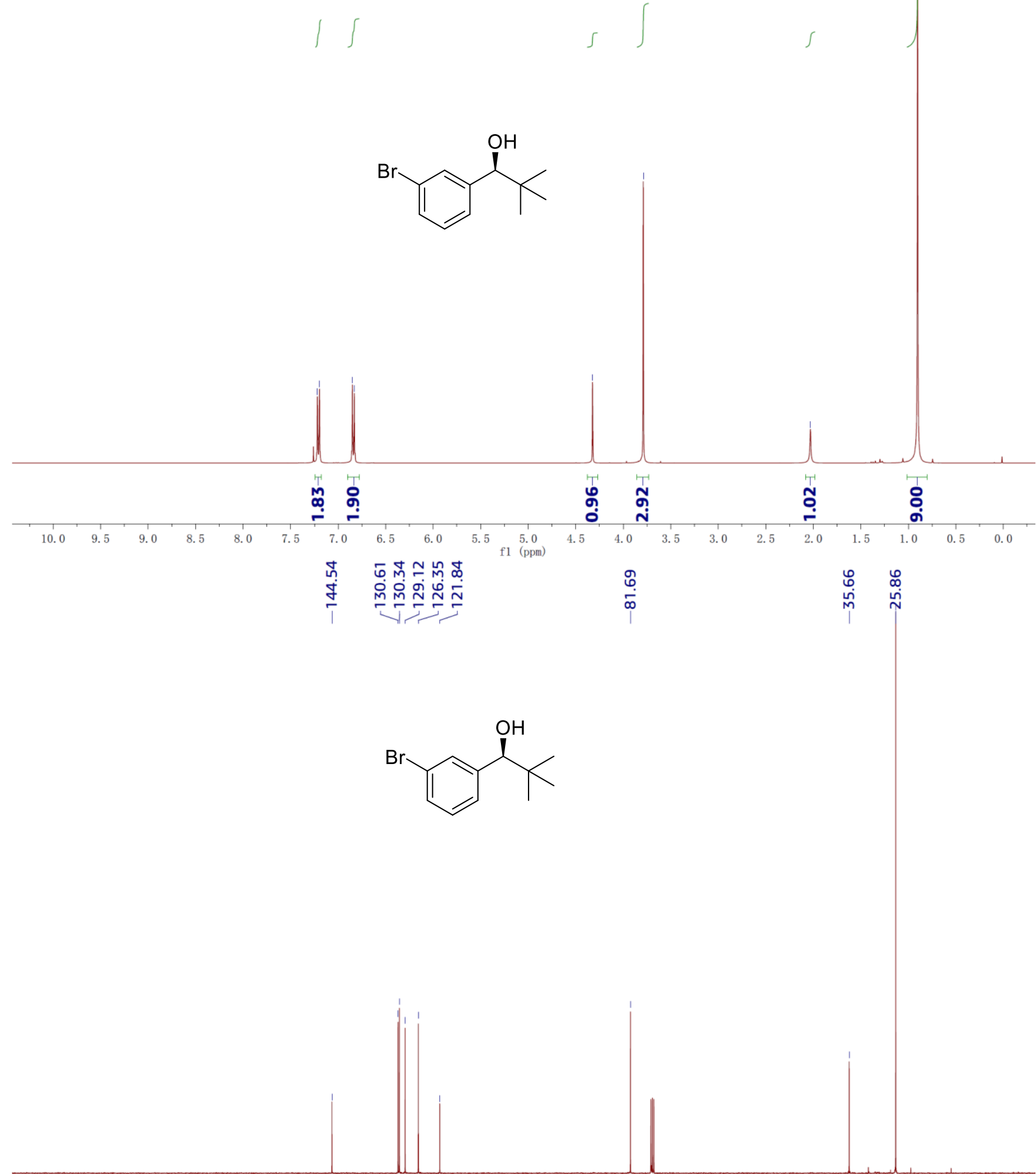

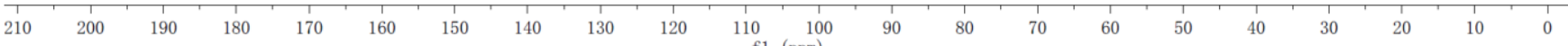


(S)-1-(4-bromophenyl)-2,2-dimethylpropan-1-ol (4f)

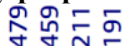

穴

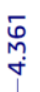

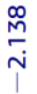

ஸ़

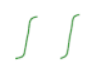<smiles>CC(C)(C)[C@@H](O)c1ccc(Br)cc1</smiles>
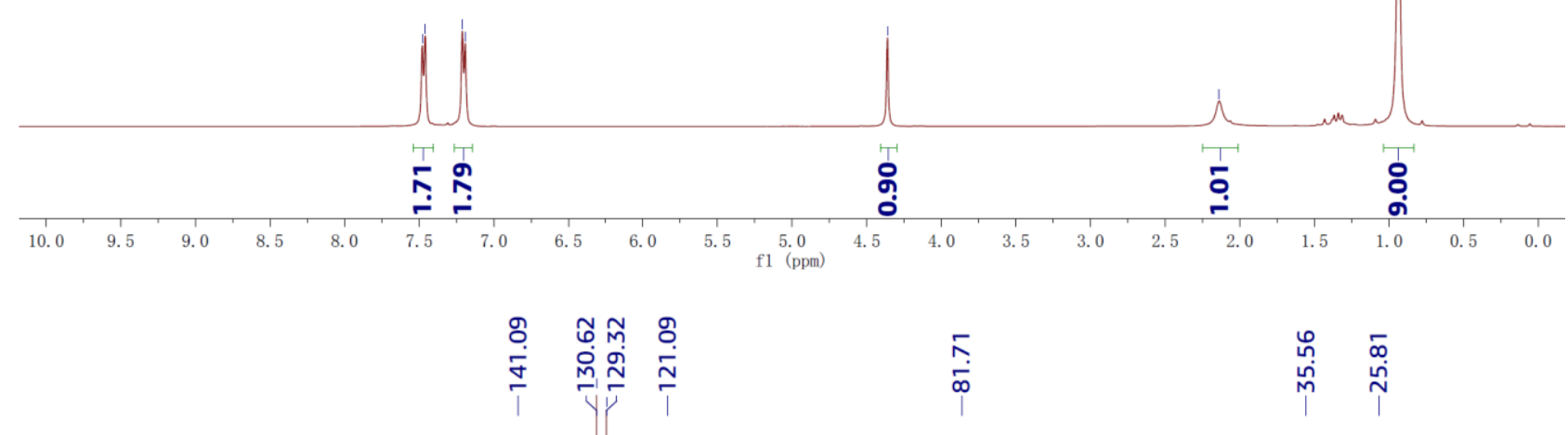

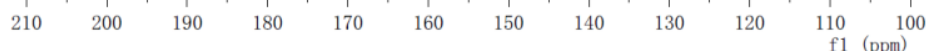

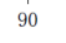

80

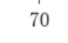

60

$\begin{array}{llllll}50 & 40 & 30 & 20 & 10 & 0\end{array}$ 
(S)-1-phenylpropan-1-ol (4g)
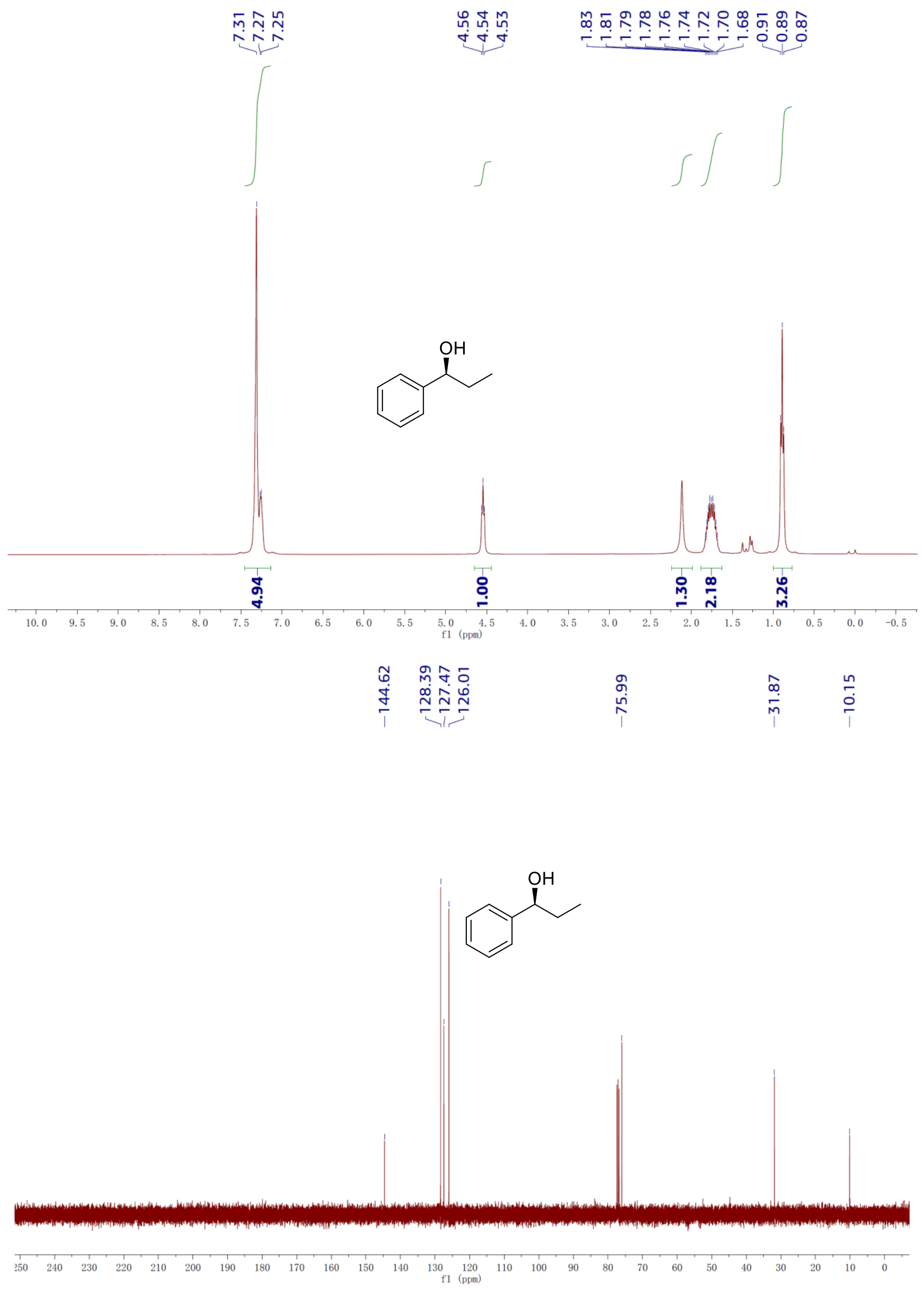
(S)-2-methyl-1-phenylpropan-1-ol (4h)

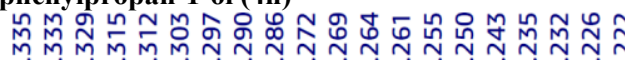

$\stackrel{\text { m. }}{\text { m. }}$

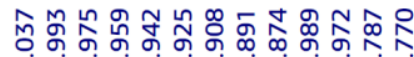

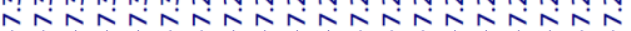

m

N- - - -
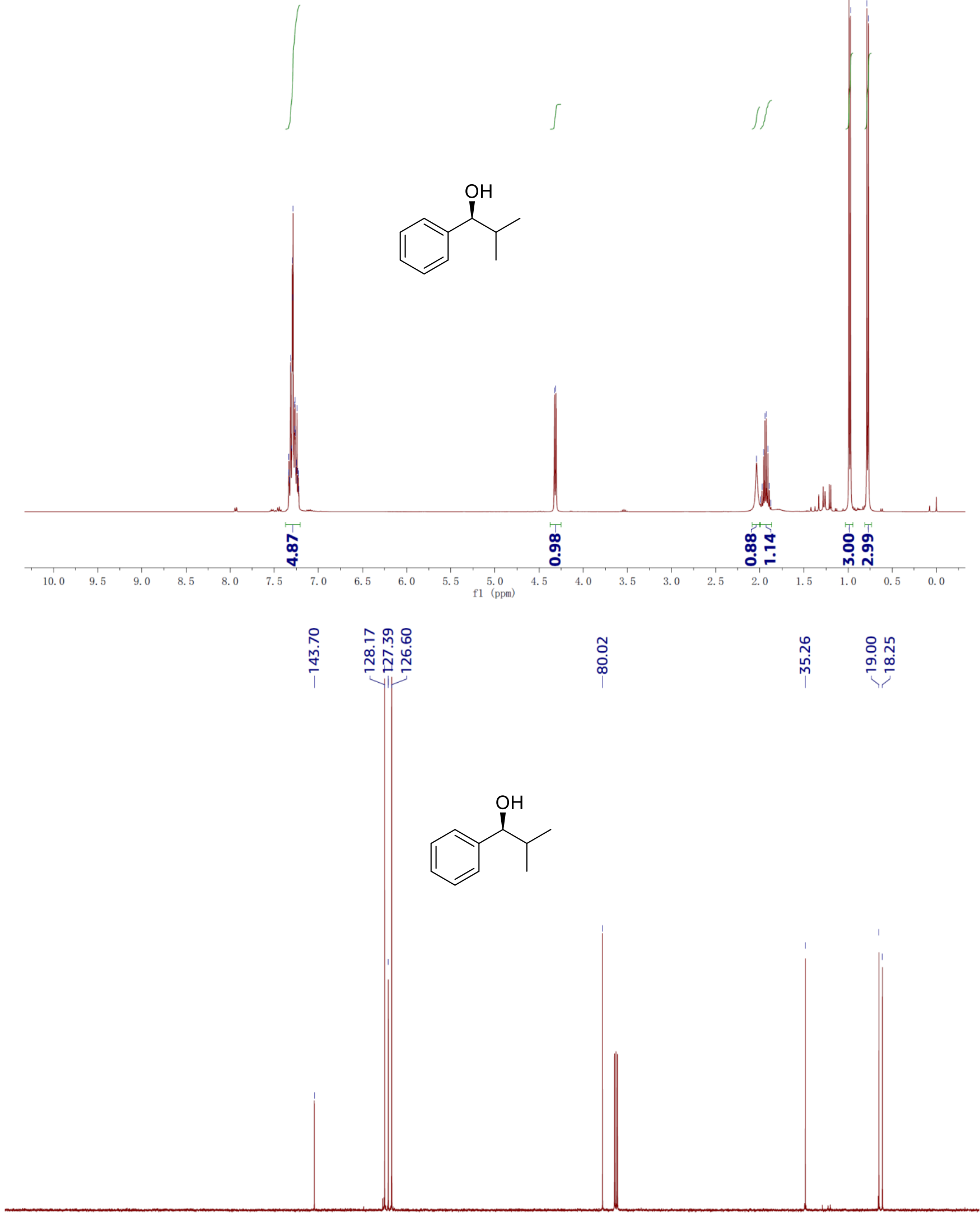
(S)-1-phenylbutan-1-ol (4i)
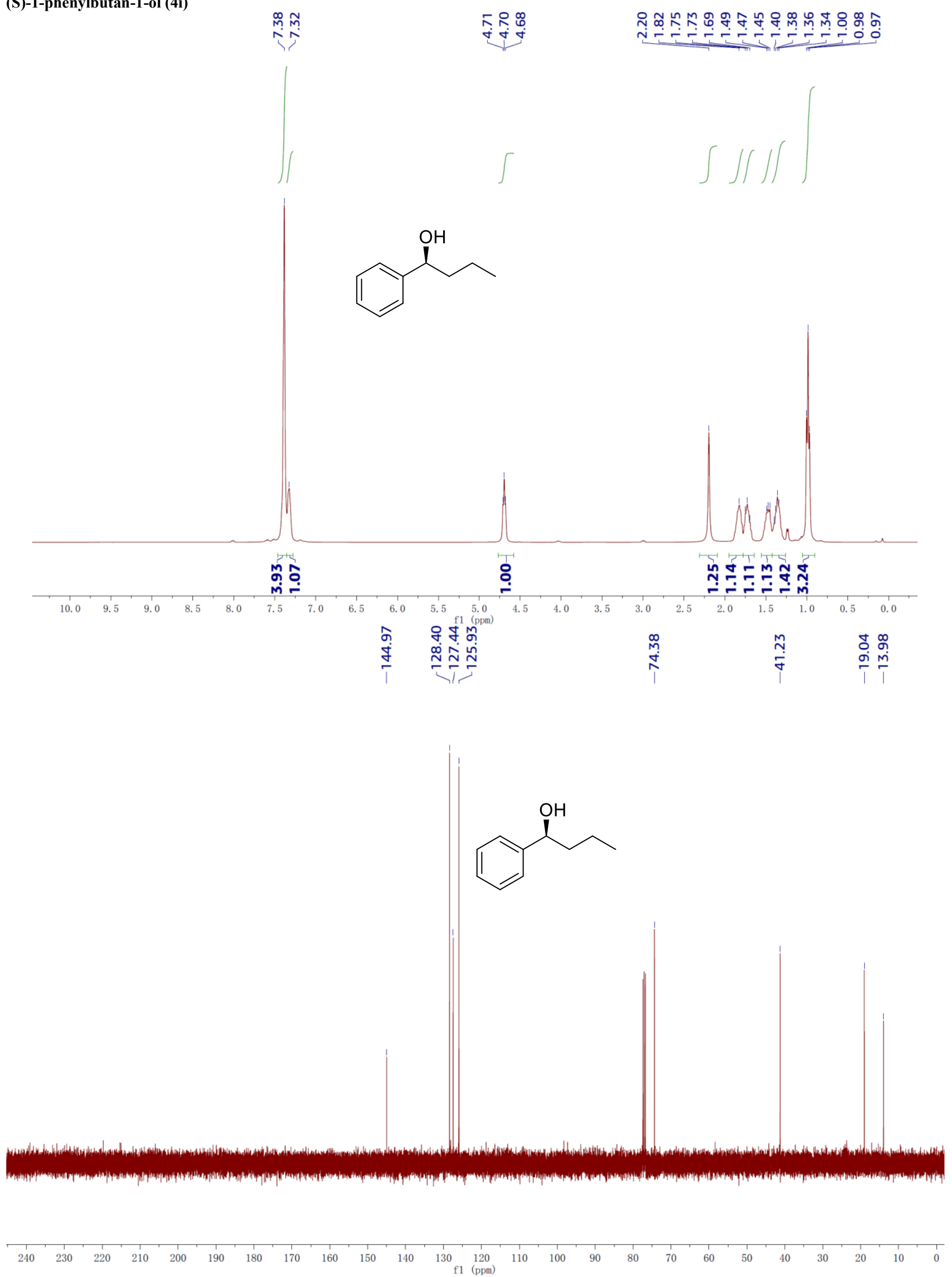
(S)-cyclopentyl(phenyl)methanol (4j)

离离

ก̃ก

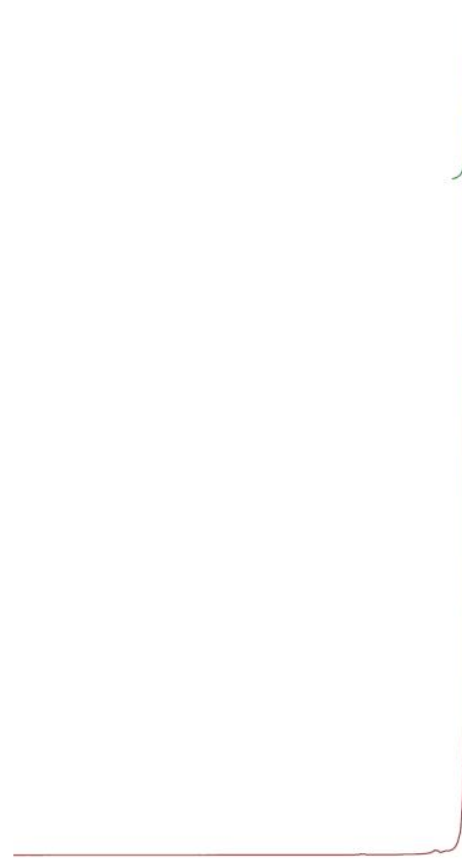

กั่

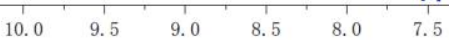

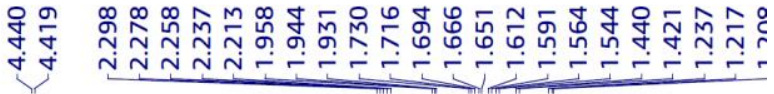<smiles>O[C@H](c1ccccc1)C1CCCC1</smiles>

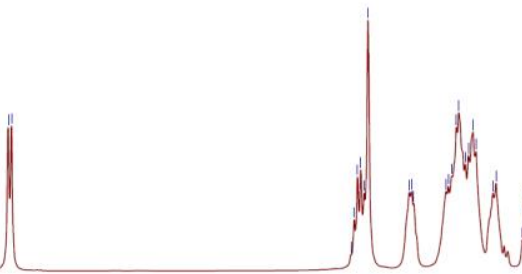

8

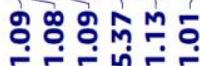

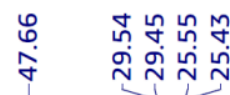<smiles>O[C@H](c1ccccc1)C1CCCC1</smiles> 
(S)-cyclohexyl(phenyl)methanol (4k)

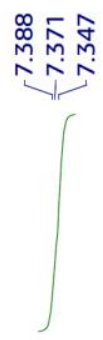

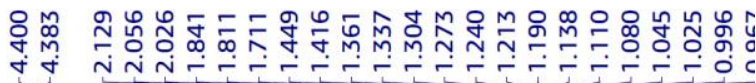

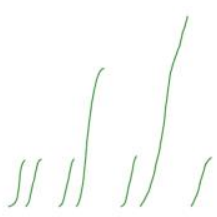<smiles>O[C@H](c1ccccc1)C1CCCCC1</smiles>
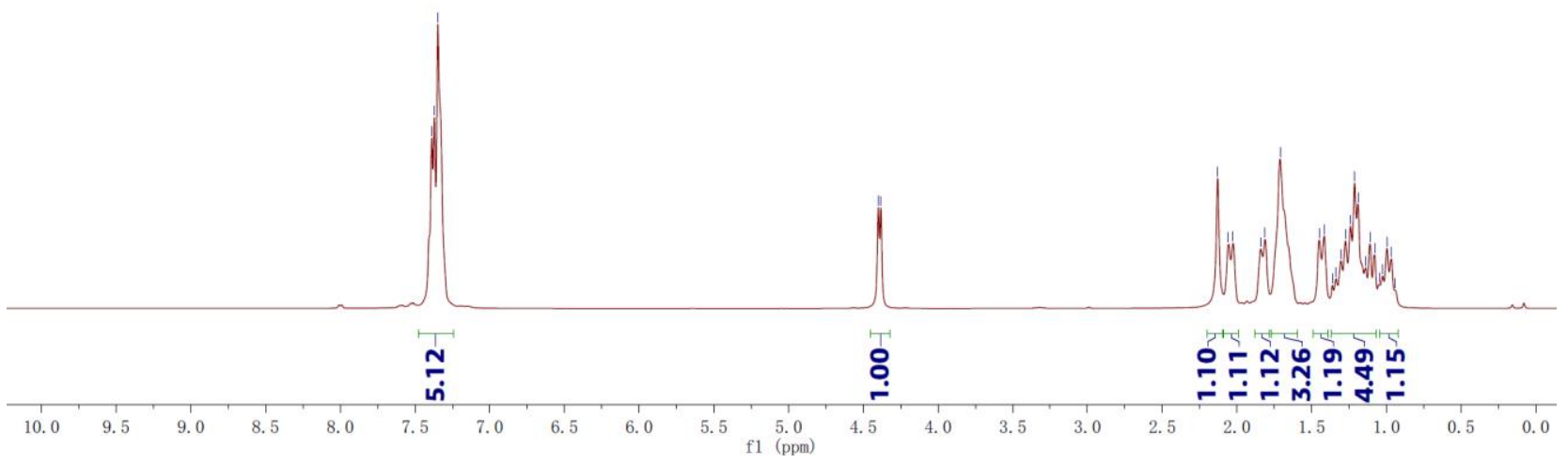

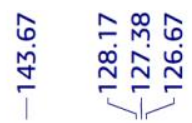

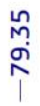

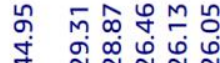

J

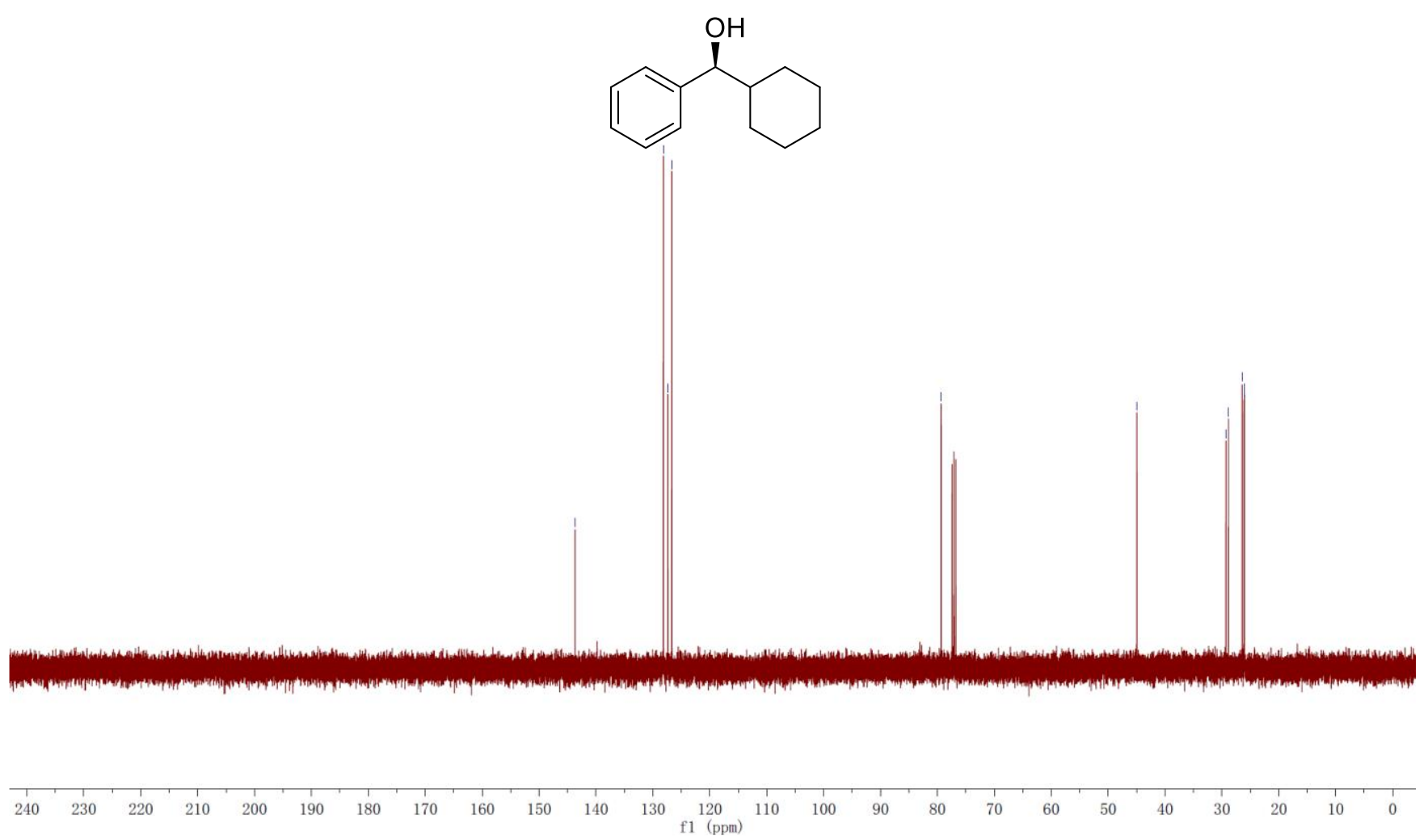


(S)-1-(2,4-dichlorophenyl)propan-1-ol (4I)

守衣药

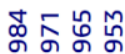

बें भु भं

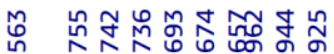

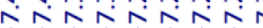

i

$\iint$
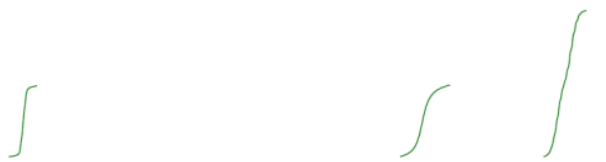<smiles>CC[C@H](O)c1ccc(Cl)cc1Cl</smiles>
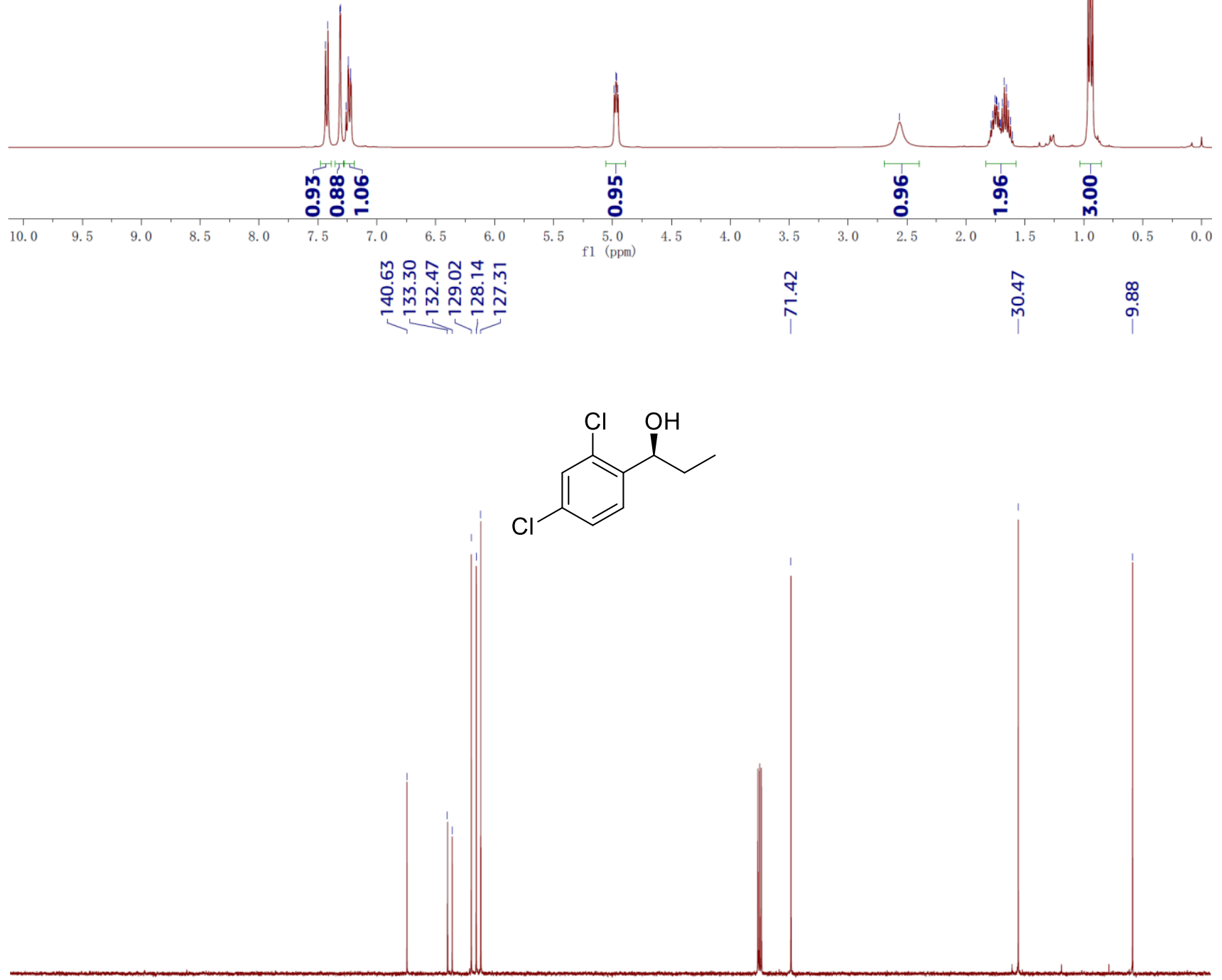

$\begin{array}{rrrrrrrrrrrrrrrrrrrrrr}\mid & \mid \\ 210 & 200 & 190 & 180 & 170 & 160 & 150 & 140 & 130 & 120 & 110 & 100 & 90 & 80 & 70 & 60 & 50 & 40 & 30 & 20 & 10 & 1\end{array}$ 


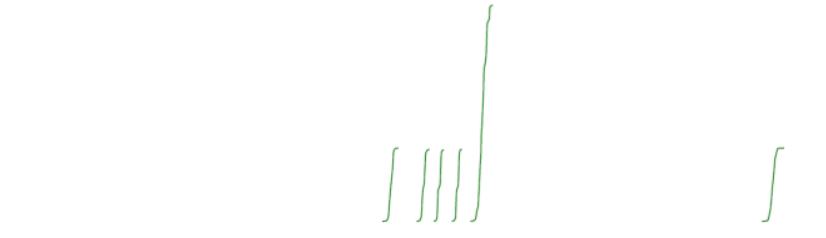<smiles>C[C@H](O)c1cccc2ccccc12</smiles><smiles>C#CC</smiles>
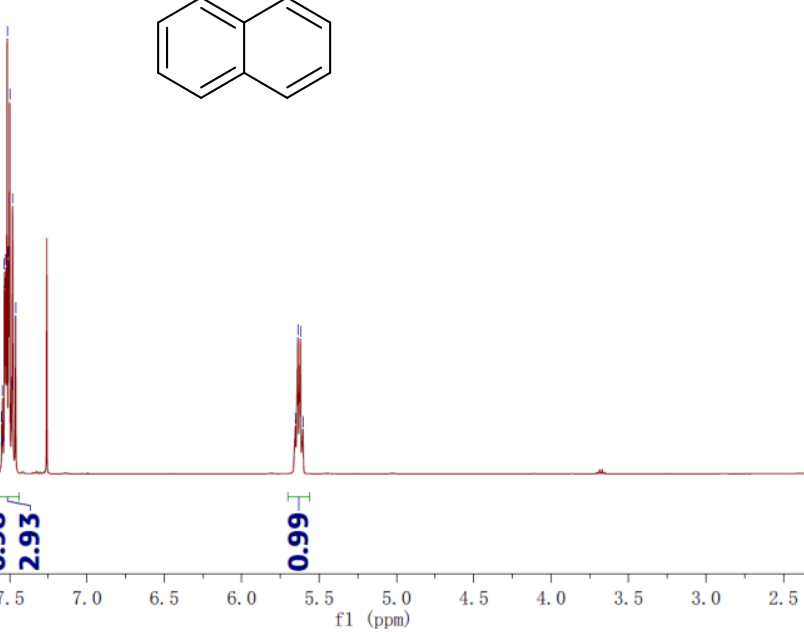

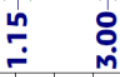

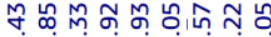

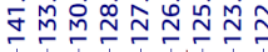

$\stackrel{0}{i}$
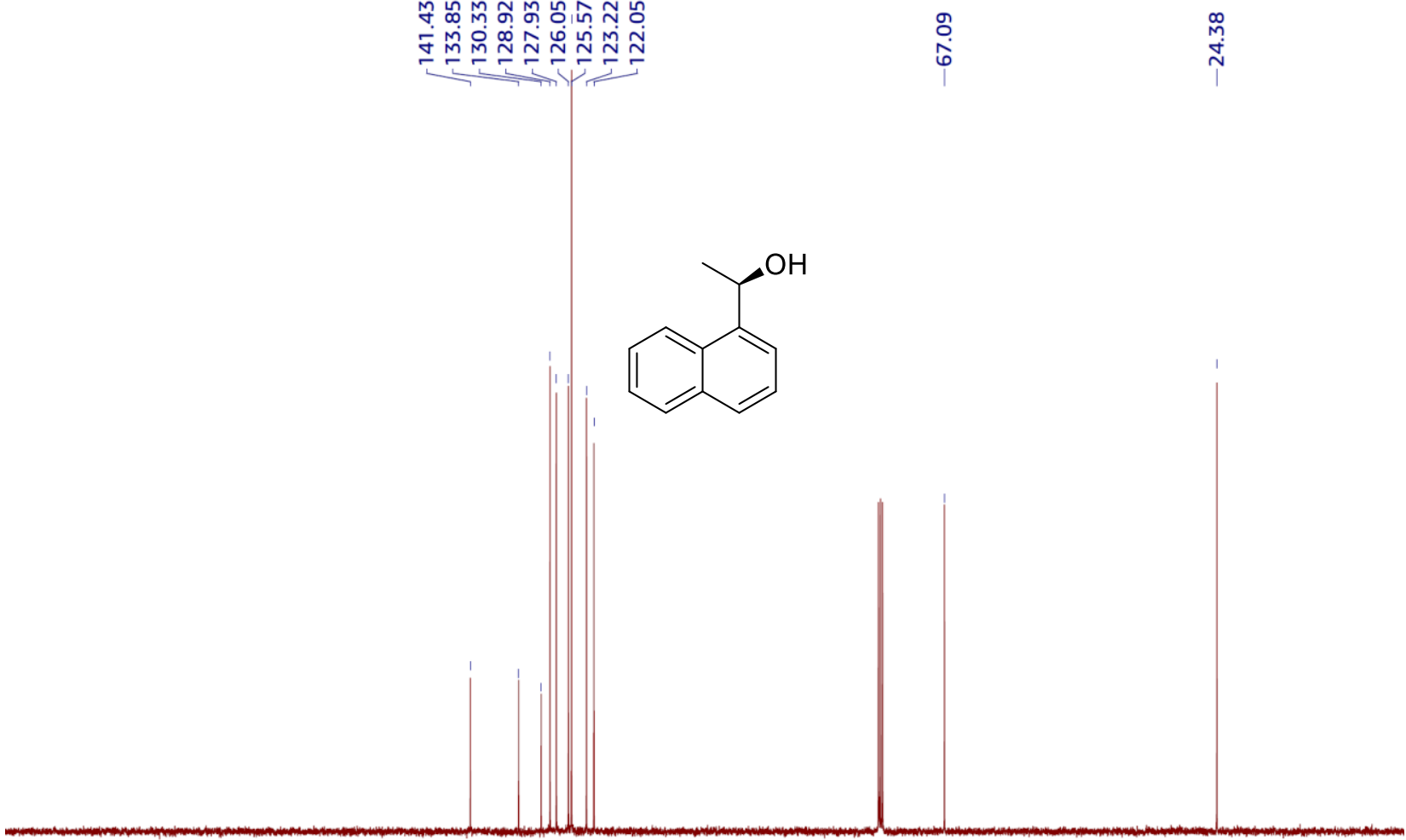

$\begin{array}{llllllllllll}210 & 200 & 190 & 180 & 170 & 160 & 150 & 140 & 130 & 120 & 110 & 100\end{array}$ 
(R)-1-(anthracen-9-yl)ethan-1-ol (4n)

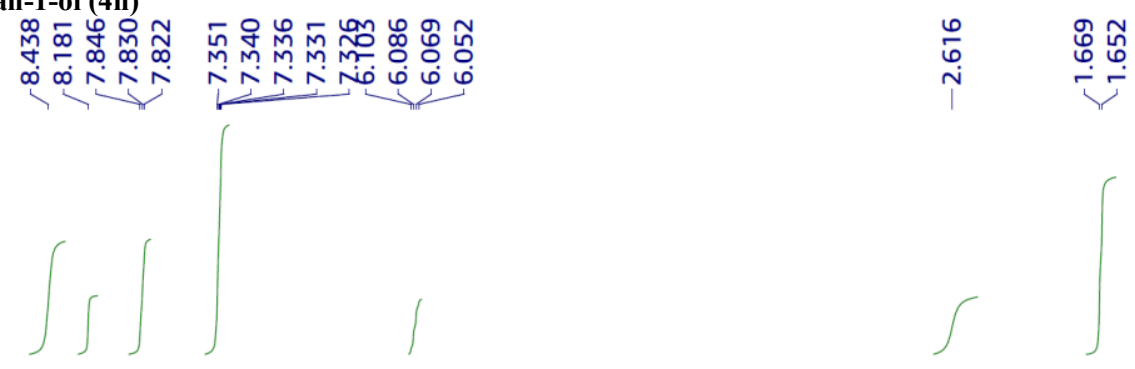<smiles>CC(O)c1c2ccccc2cc2ccccc12</smiles>

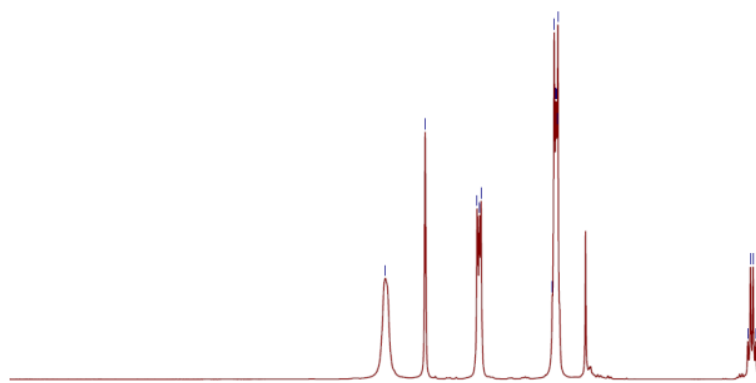

कุดัด ฏ

然

ริ :
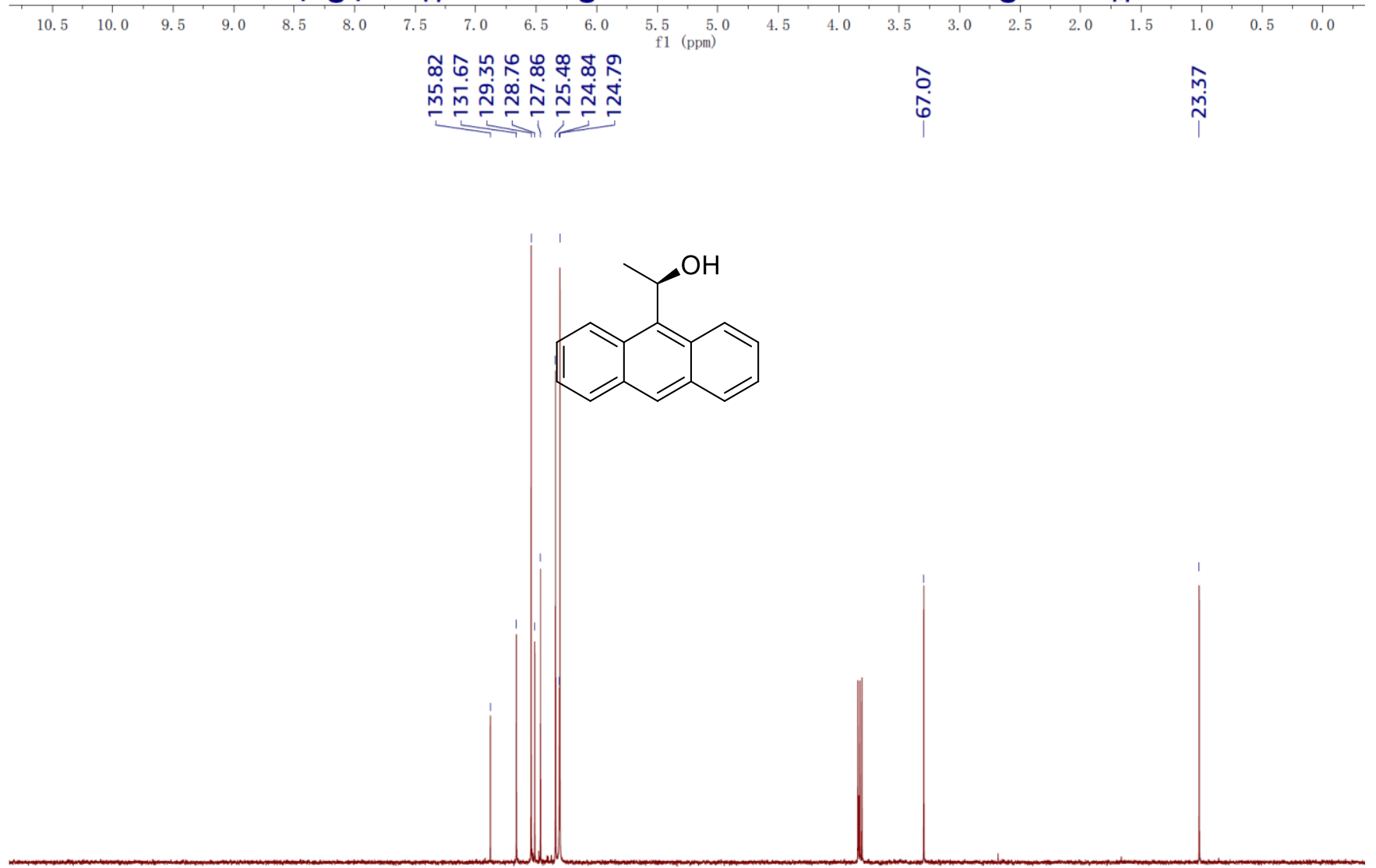

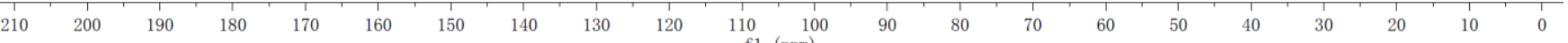




\section{Copies of GC and HPLC specta}

(S)-1-phenylethan-1-ol (2a)

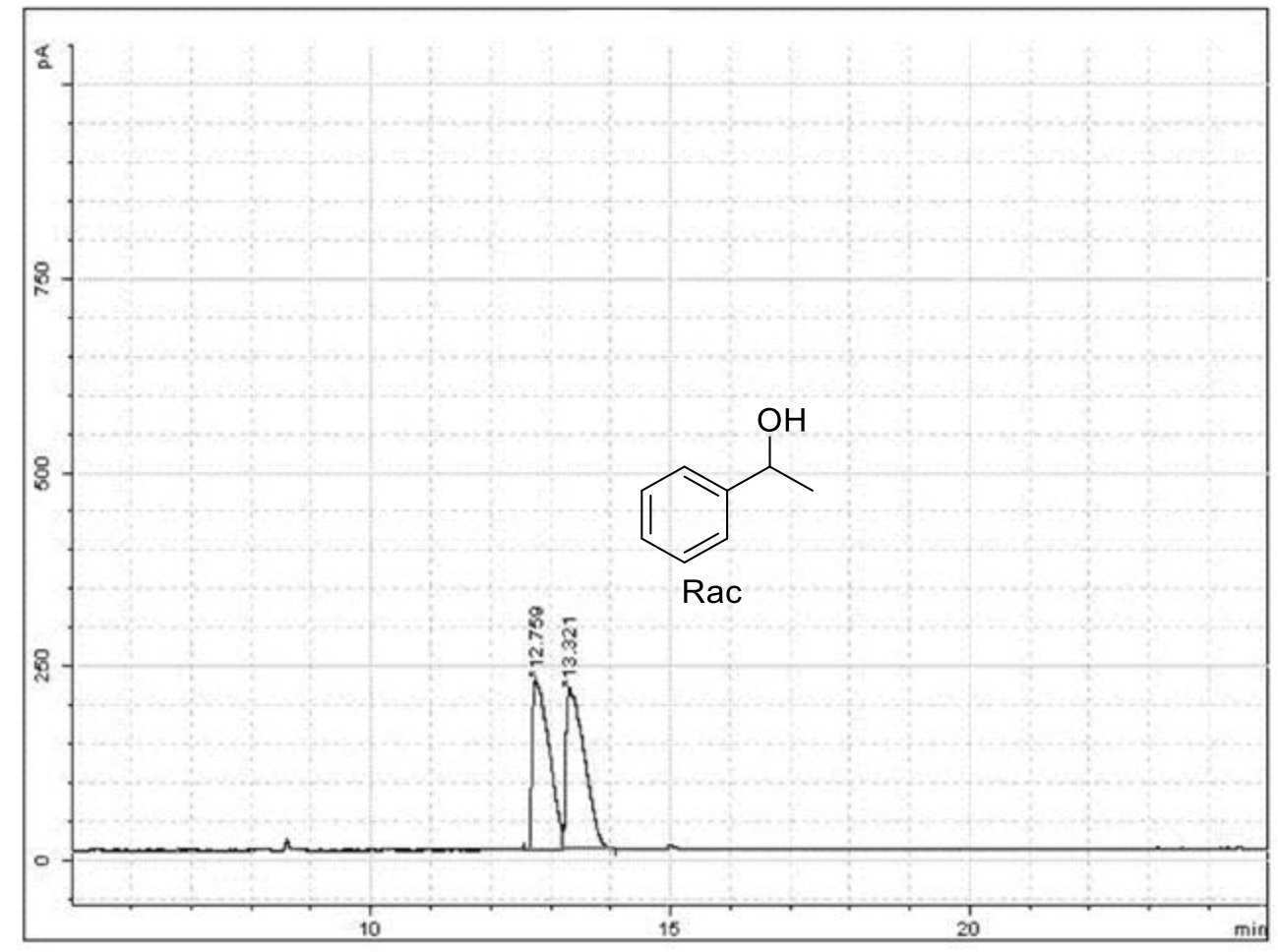

\begin{tabular}{|c|c|c|c|}
\hline Peak & Ret. Time & Area & Area \% \\
\hline 1 & 12.759 & 4322.00 & 49.21 \\
\hline 2 & 13.321 & 4460.25 & 50.79 \\
\hline
\end{tabular}

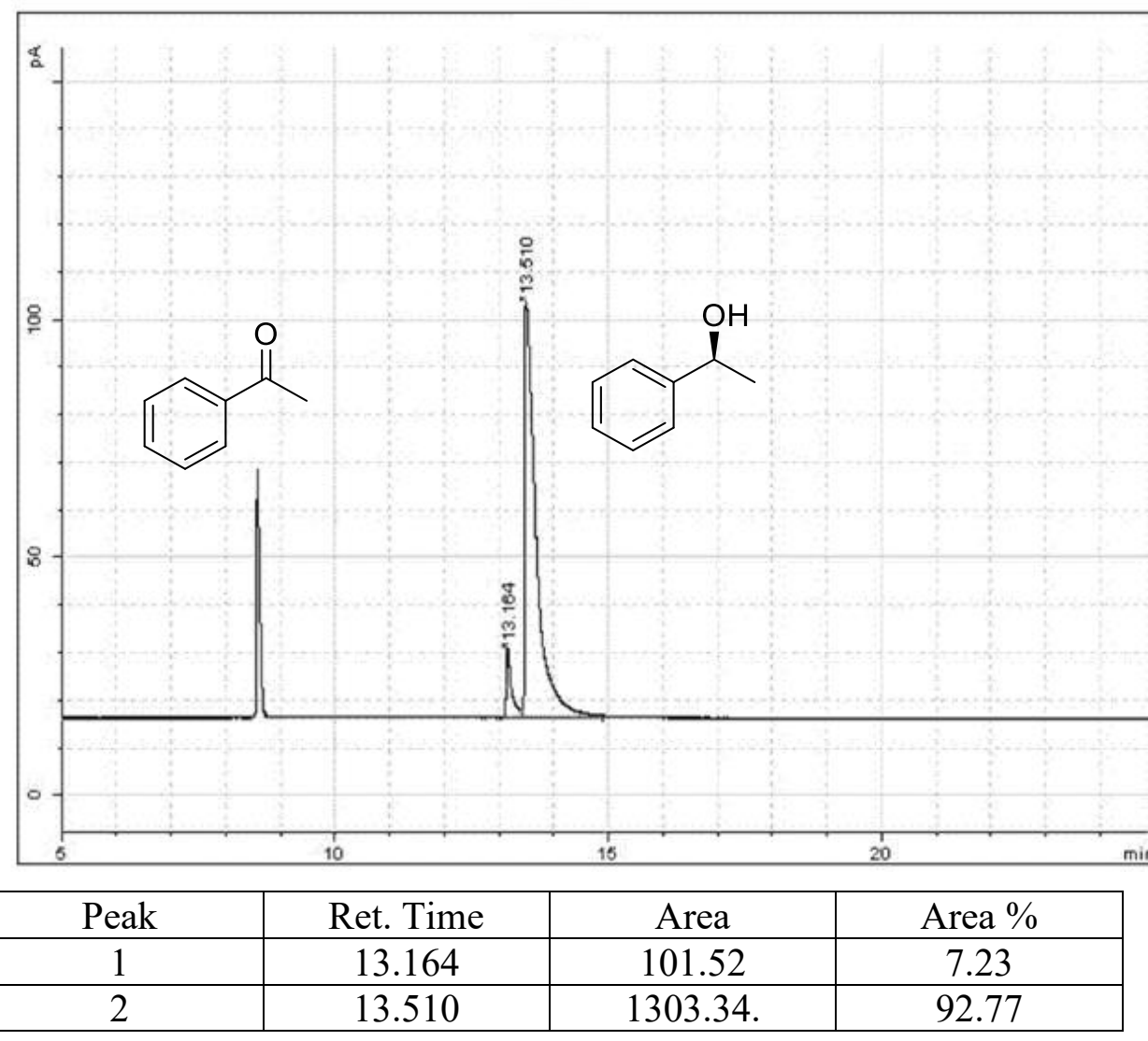



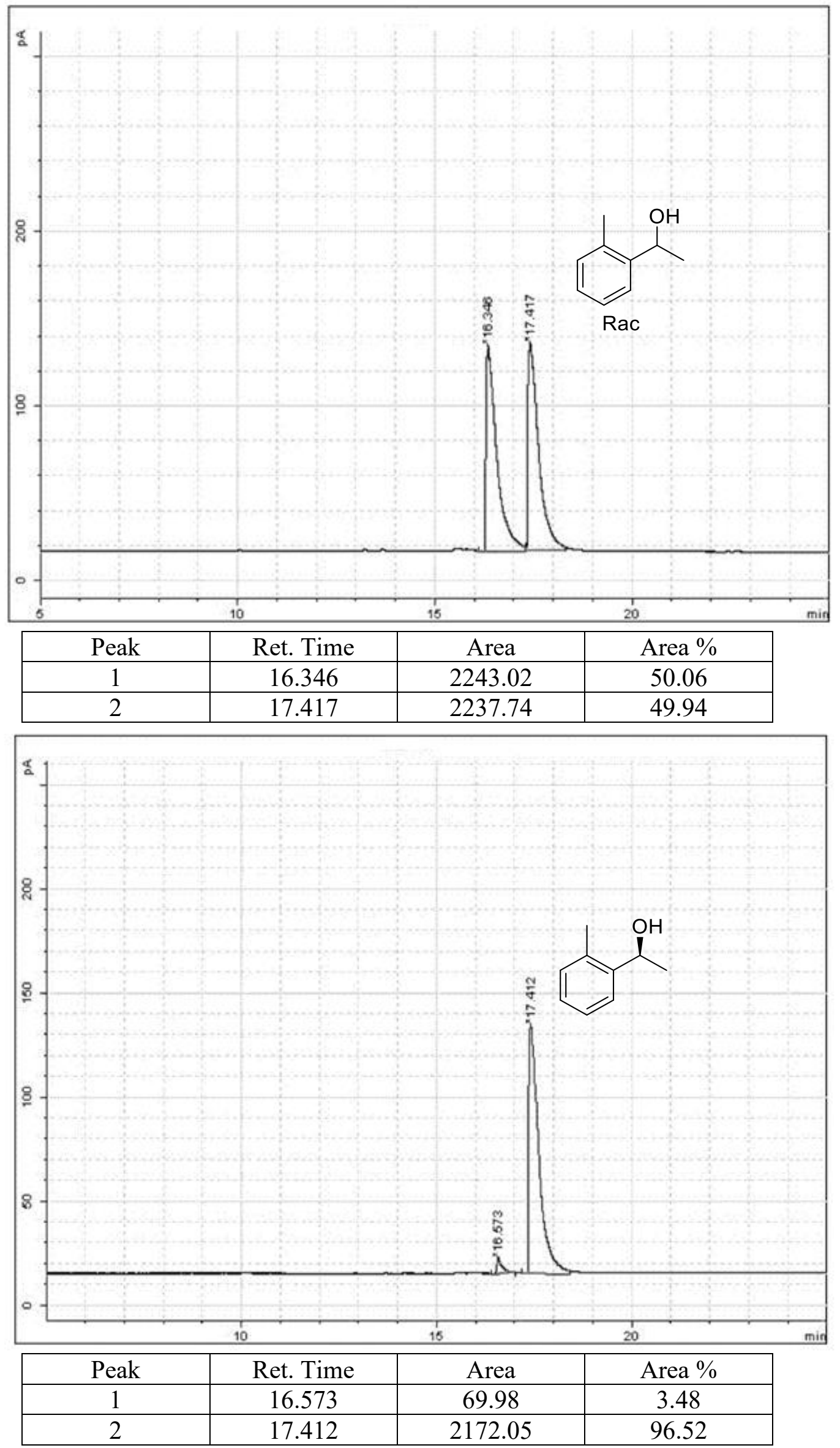
(S)-1-(2-methoxyphenyl)ethan-1-ol (2c)

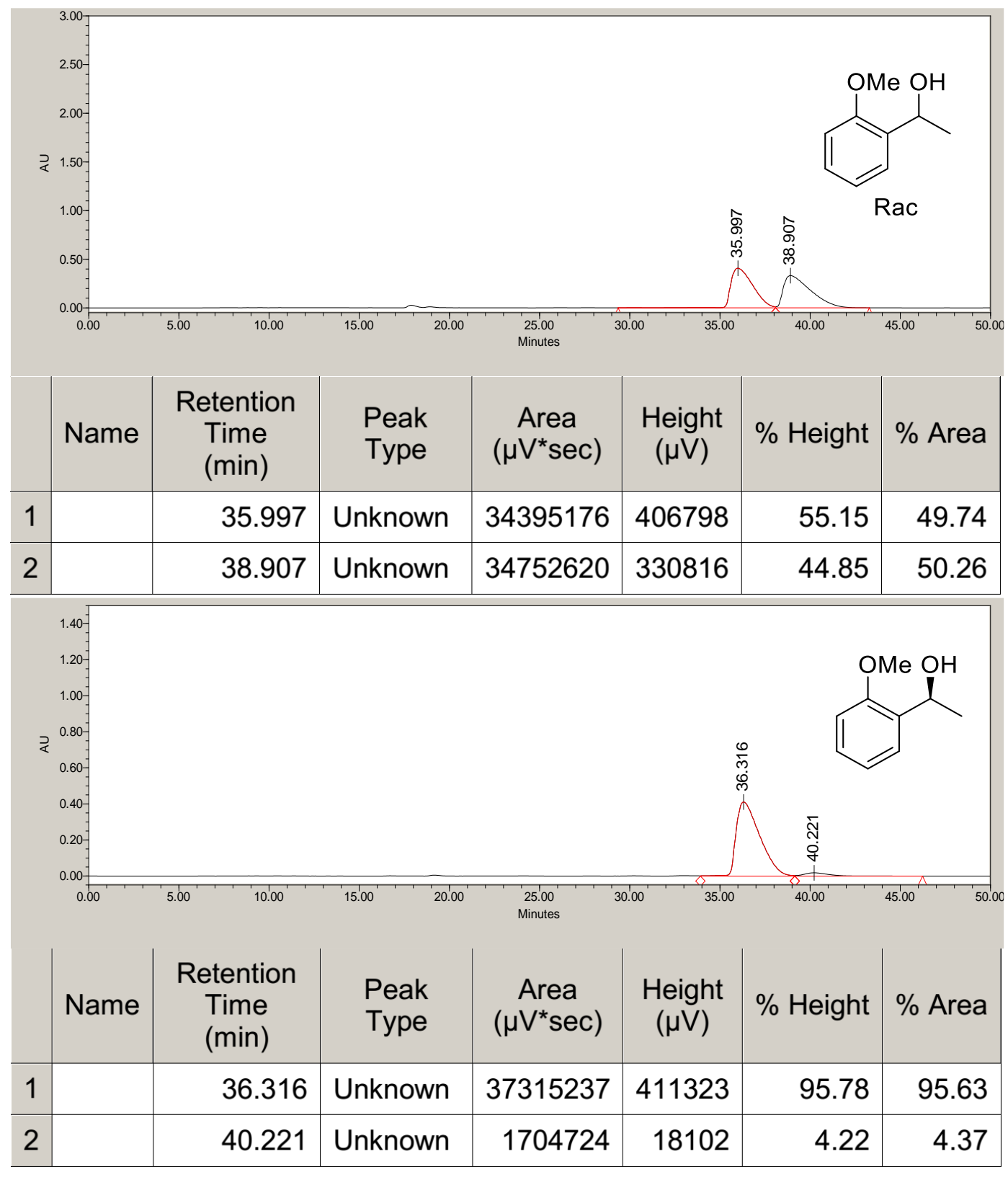




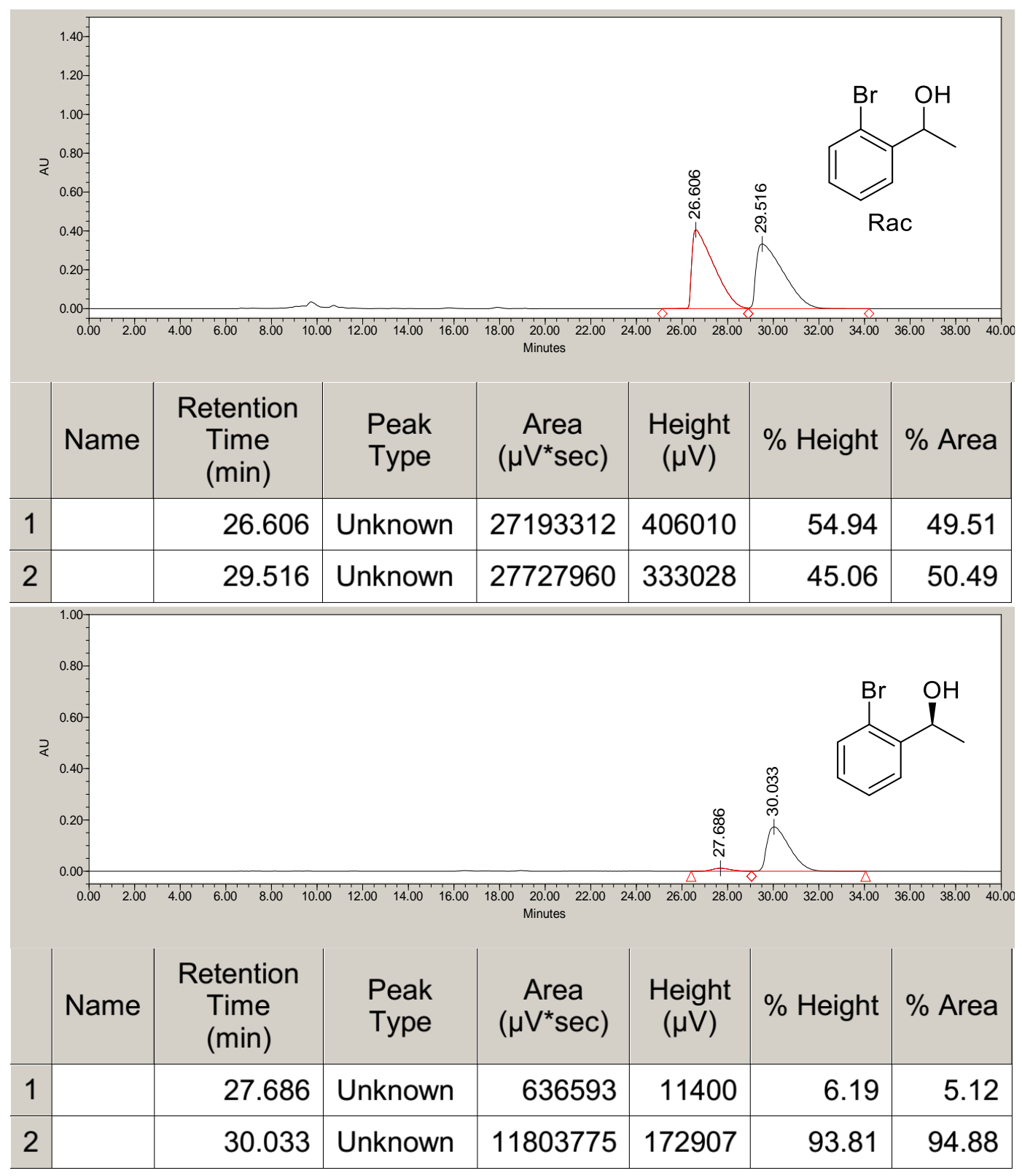


(S)-1-(4-bromophenyl)ethan-1-ol (2e)

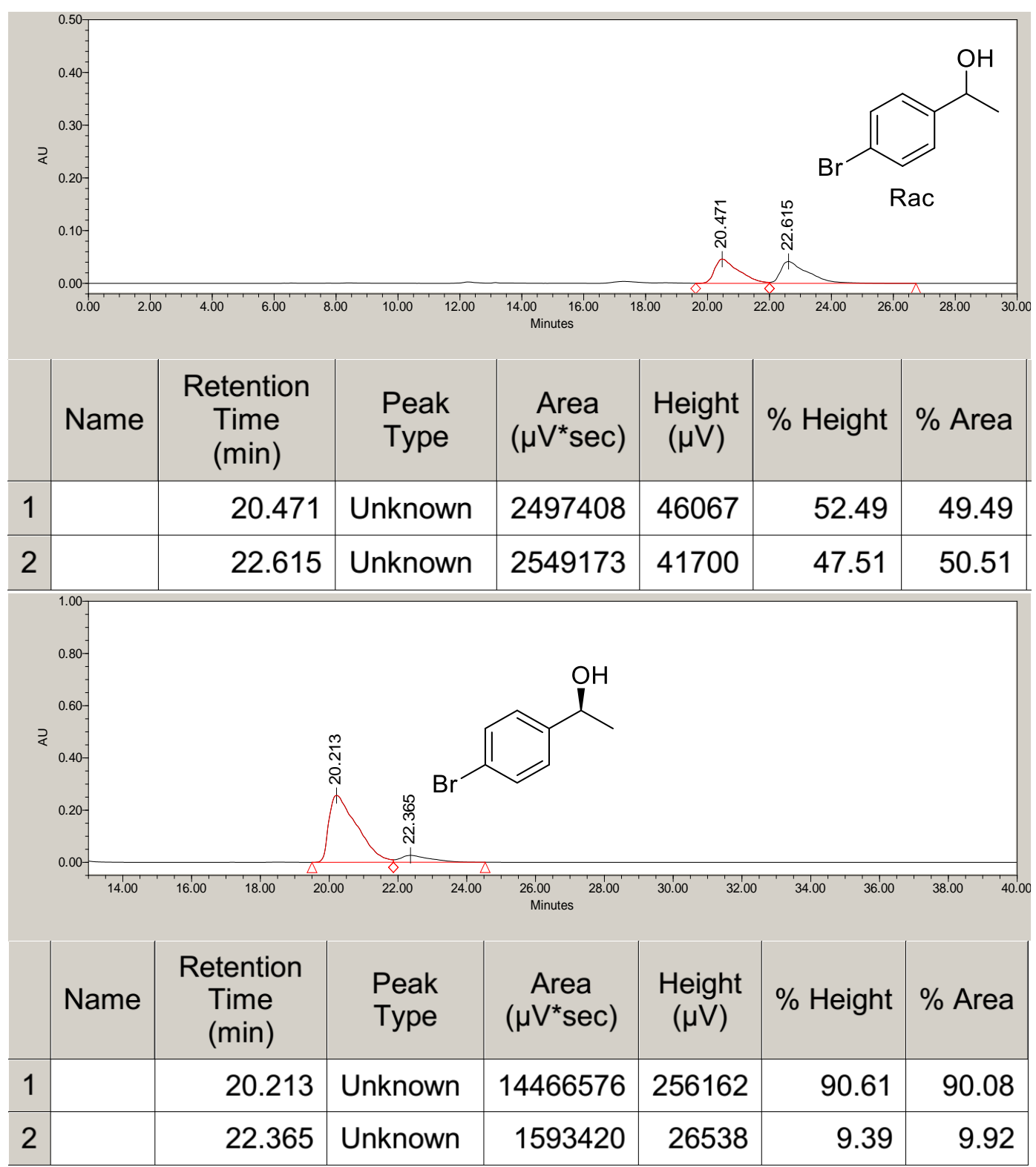



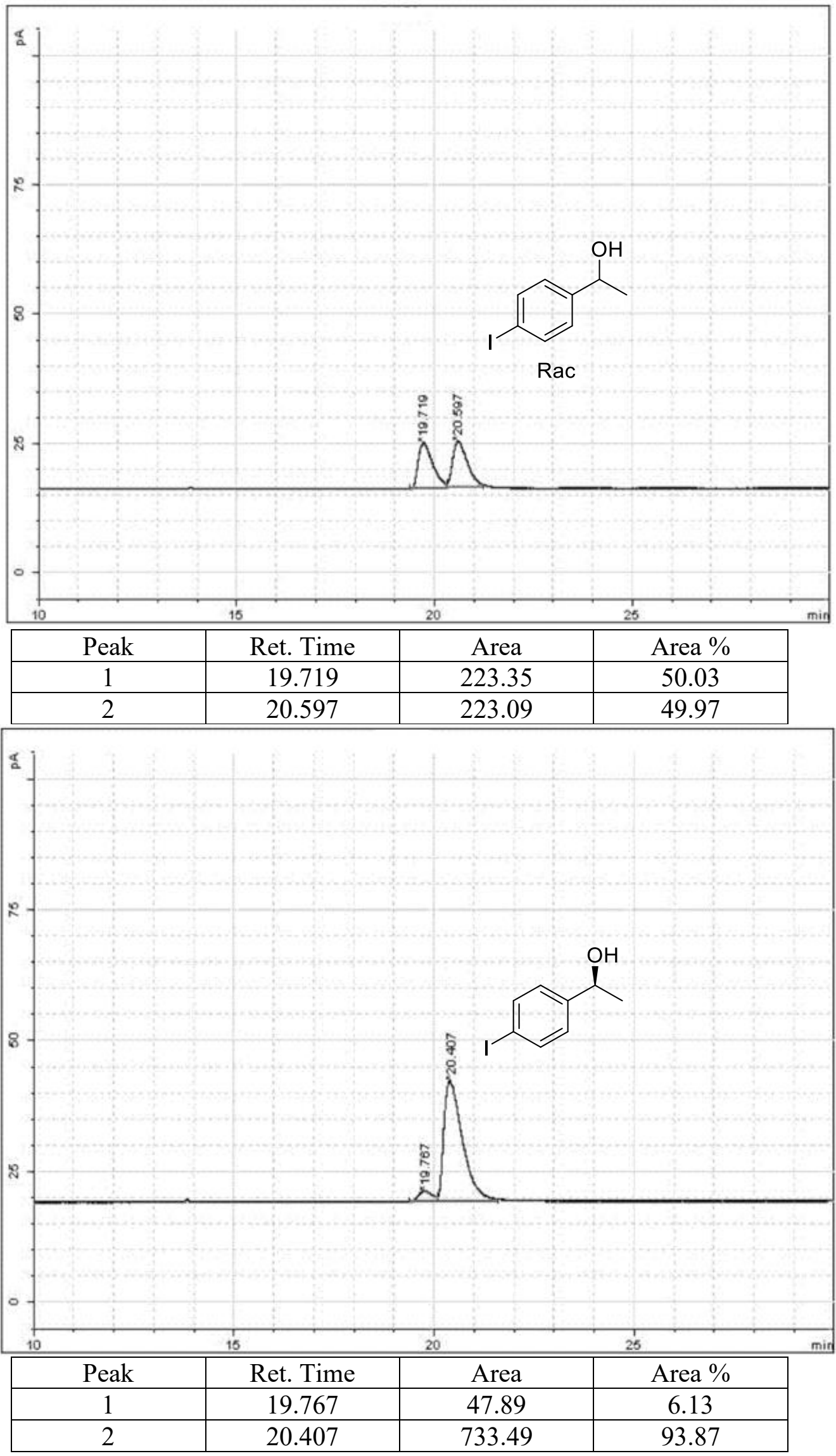

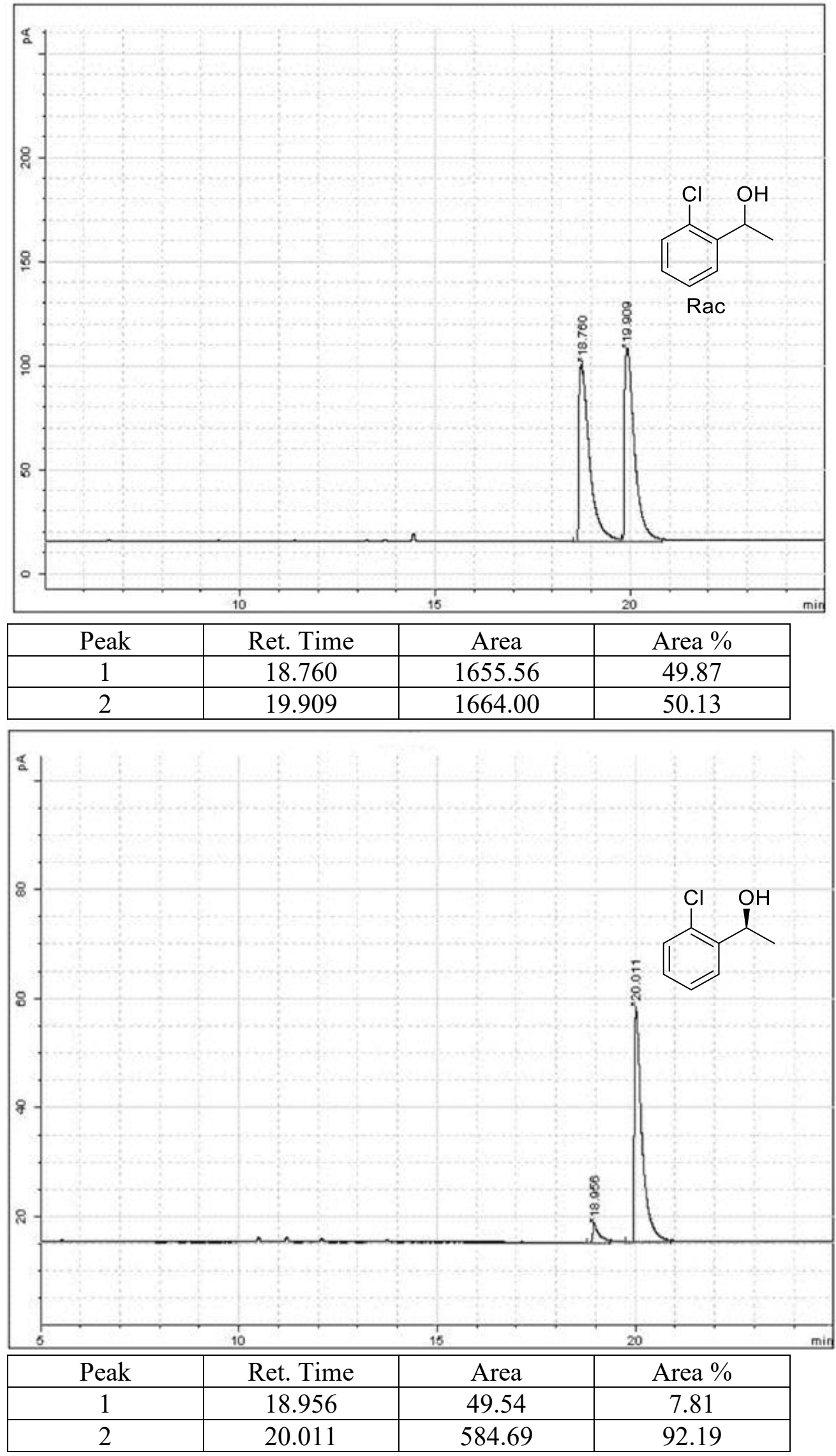

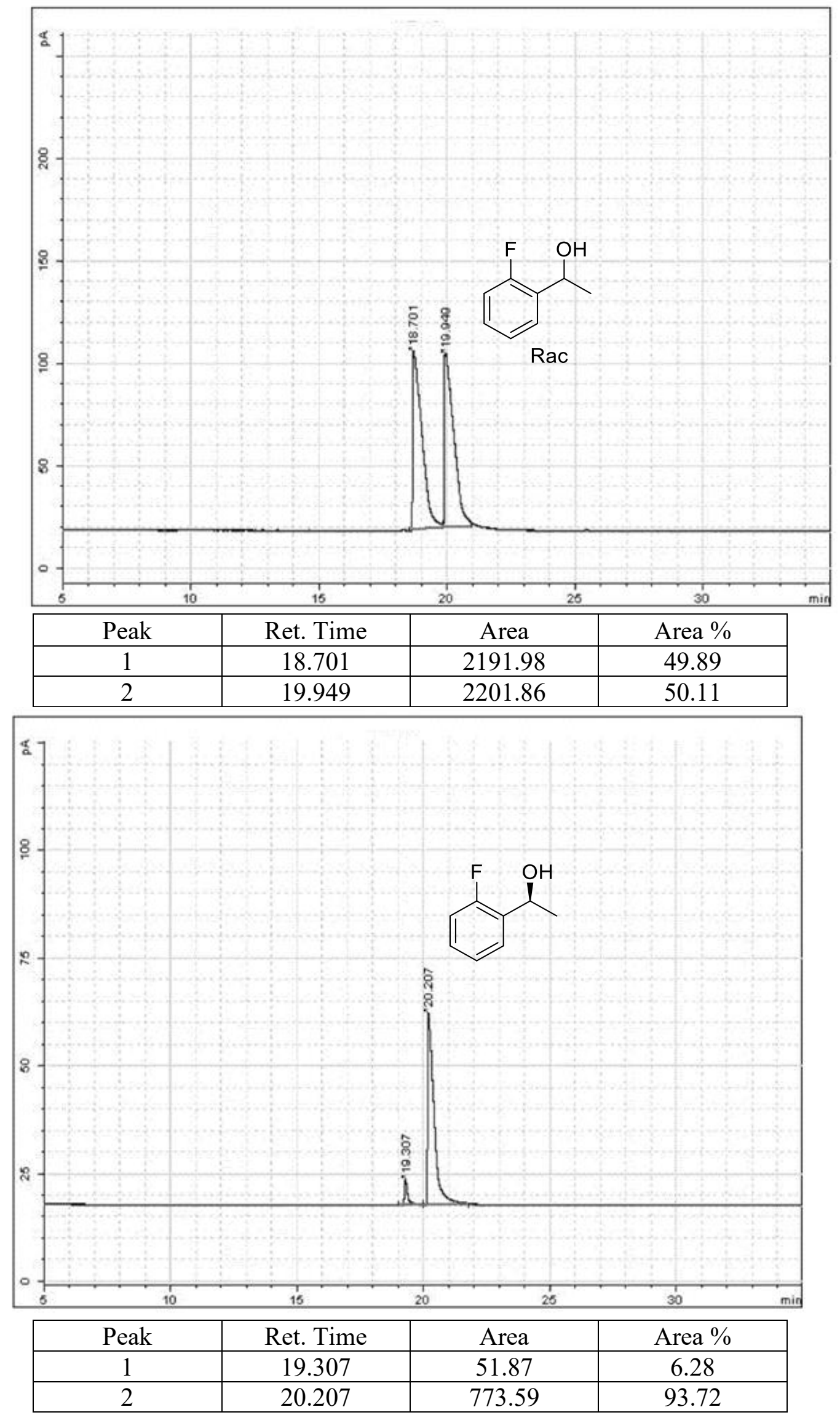

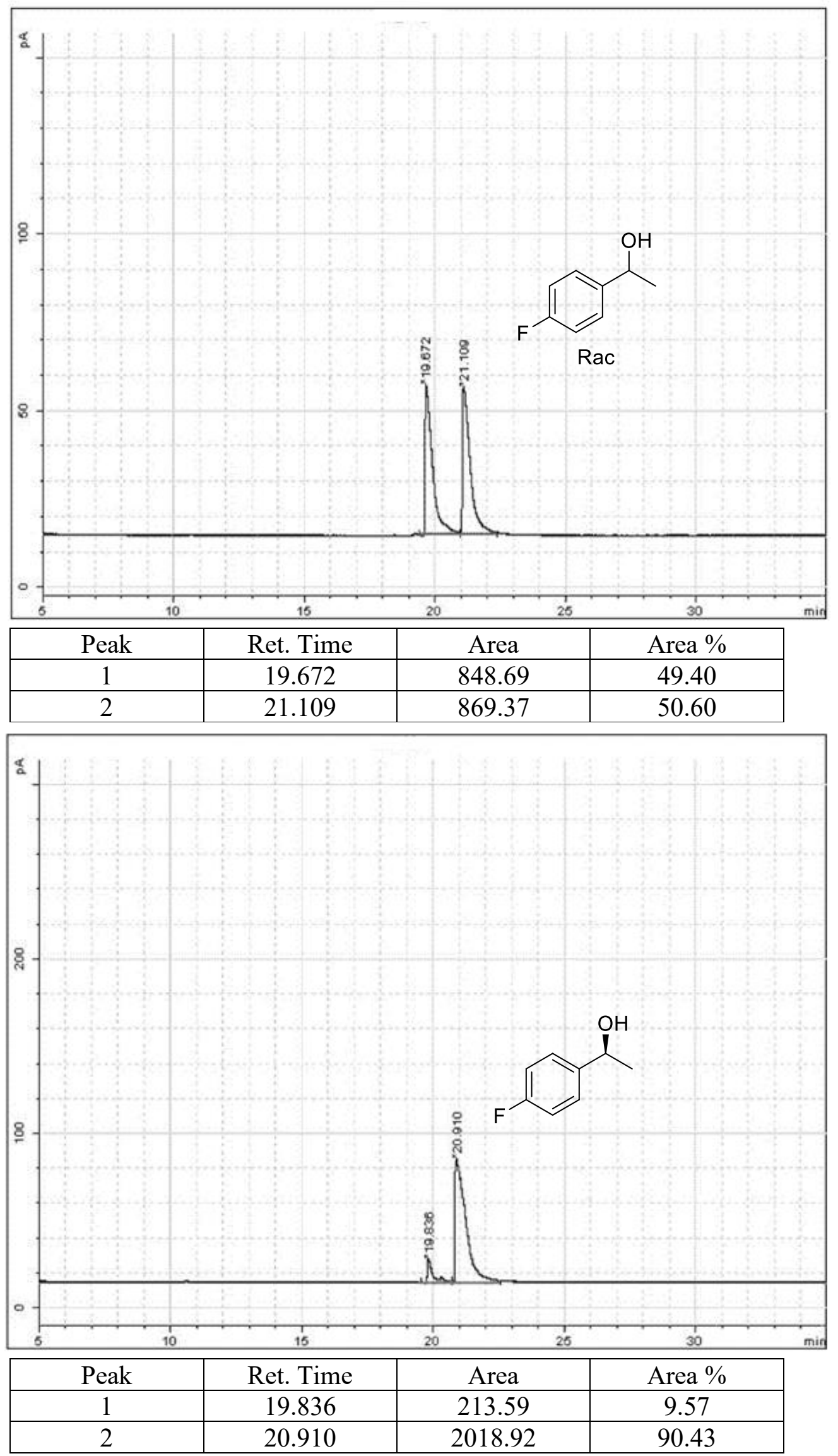

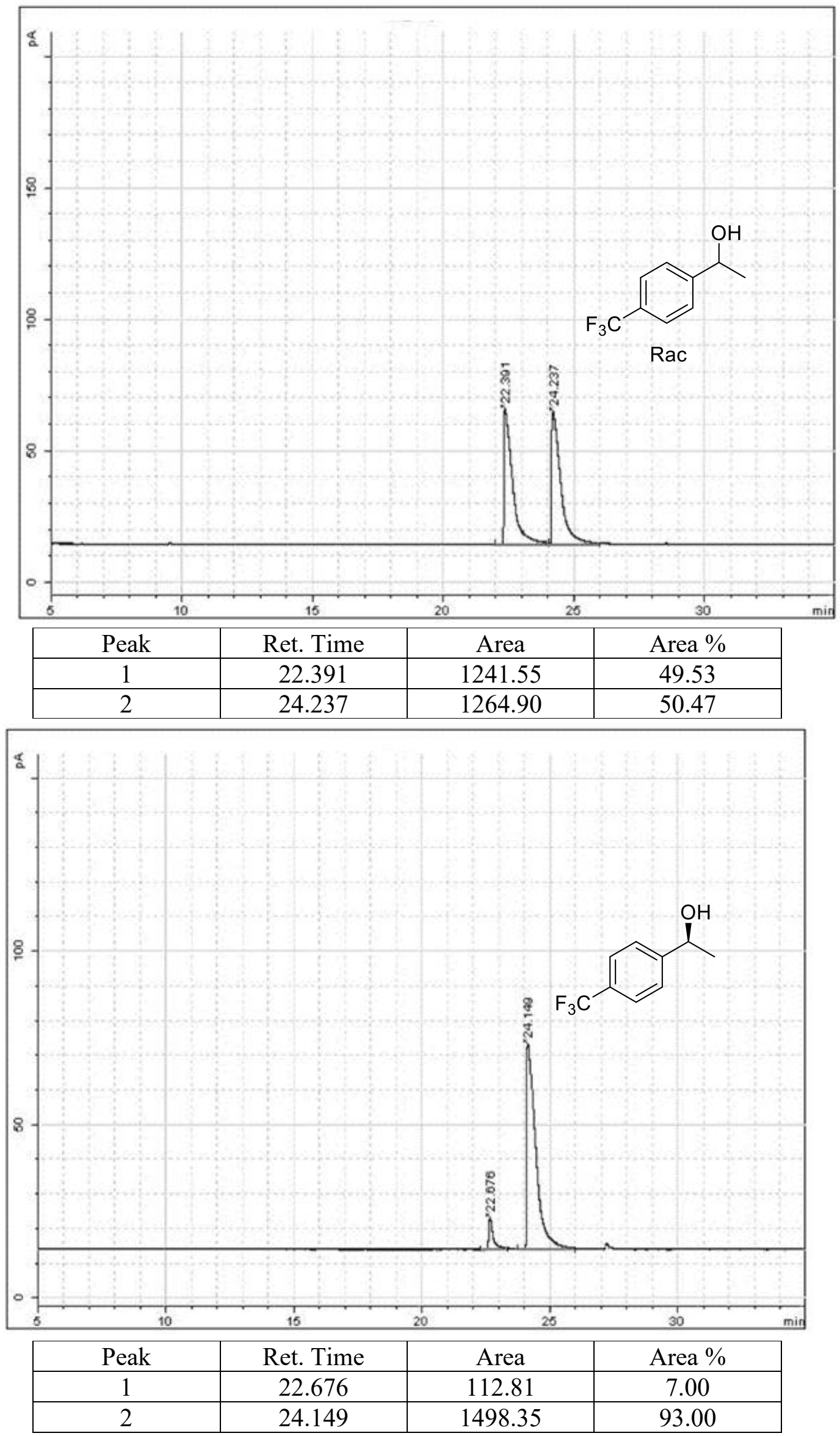

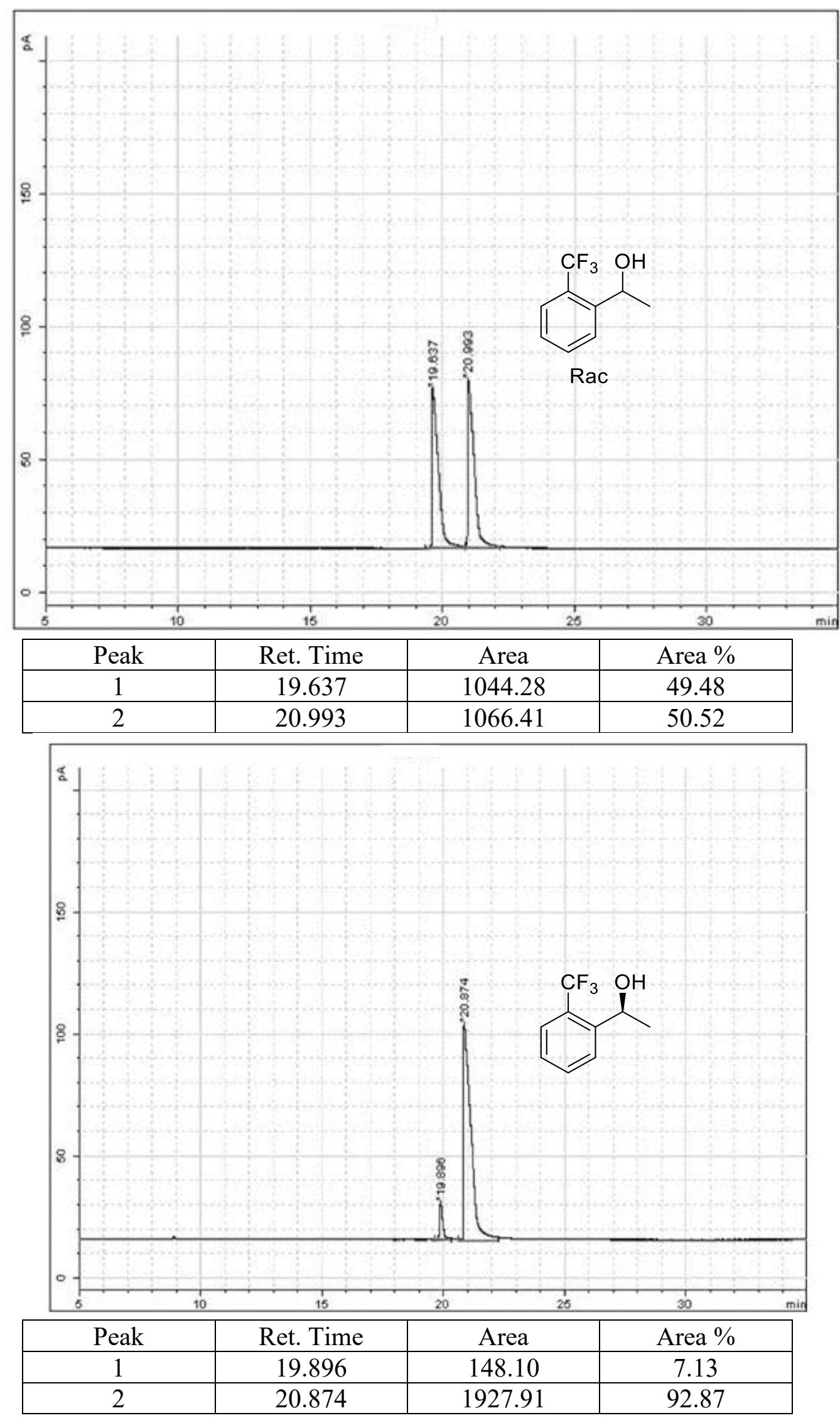
(S)-4-(1-hydroxyethyl)benzonitrile (2j)

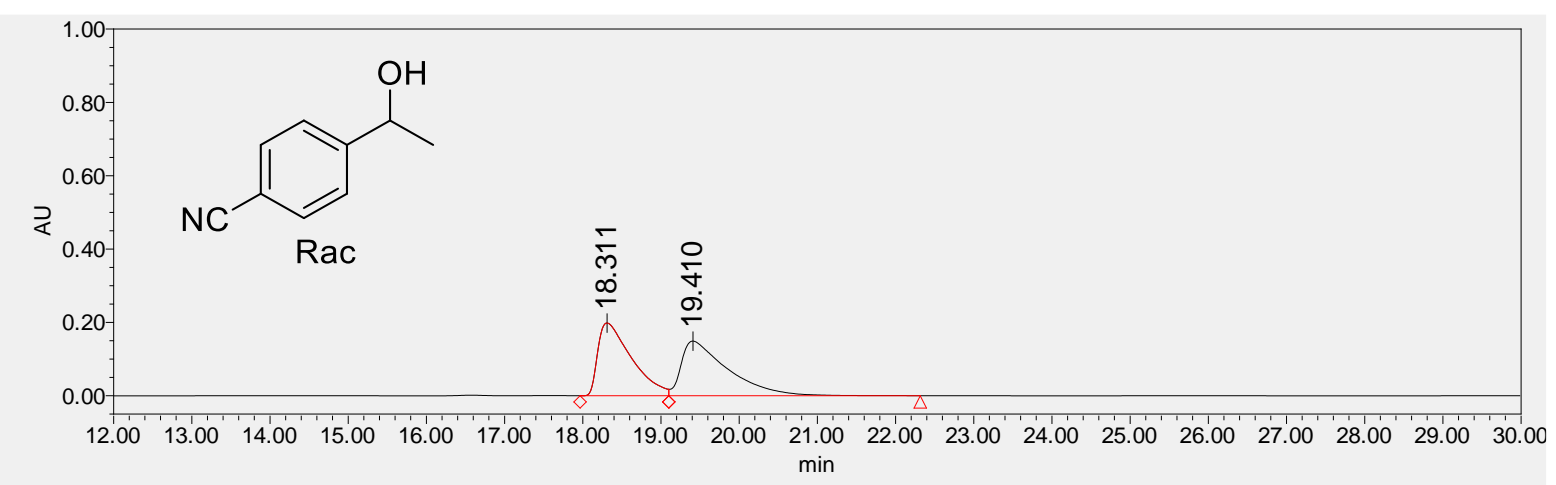

\begin{tabular}{|c|c|c|c|c|}
\hline Peak & Time & Area & Height & Area\% \\
\hline 1 & 18.311 & 5926362 & 198573 & 49.02 \\
\hline 2 & 19.410 & 6164004 & 148998 & 50.98 \\
\hline
\end{tabular}

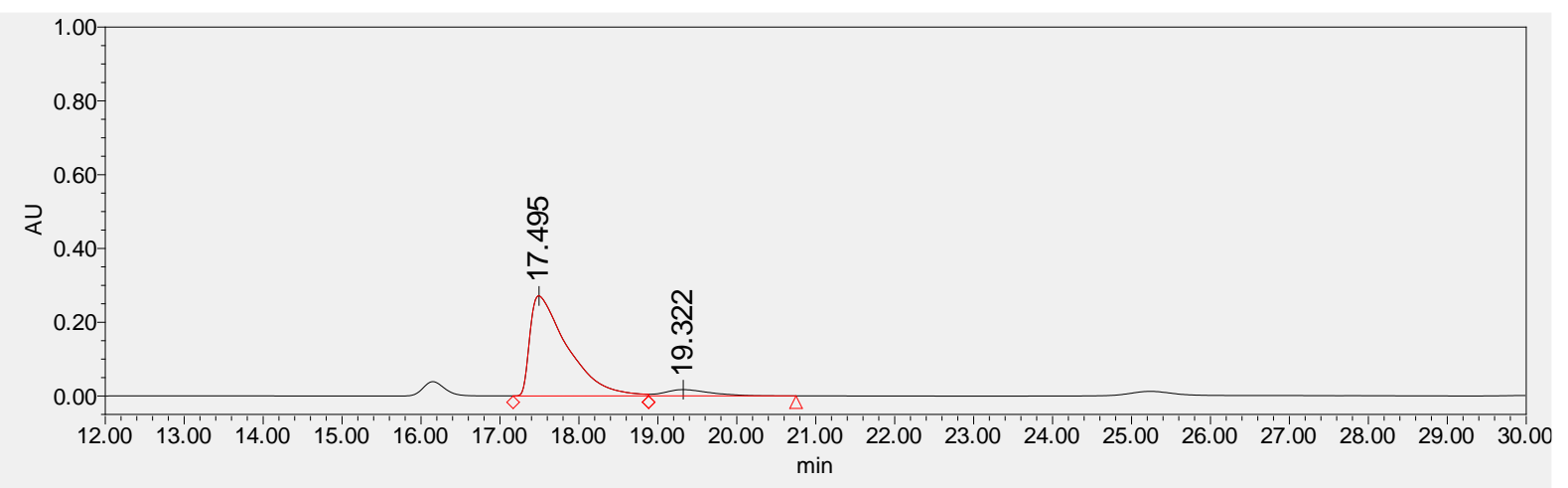

\begin{tabular}{|c|c|c|c|c|}
\hline Peak & Time & Area & Height & Area $\%$ \\
\hline 1 & 17.495 & 8968800 & 271466 & 92.80 \\
\hline 2 & 19.322 & 696076 & 17139 & 7.20 \\
\hline
\end{tabular}


(S)-1-(2,6-dichloro-3-fluorophenyl)ethan-1-ol (2m)

mV

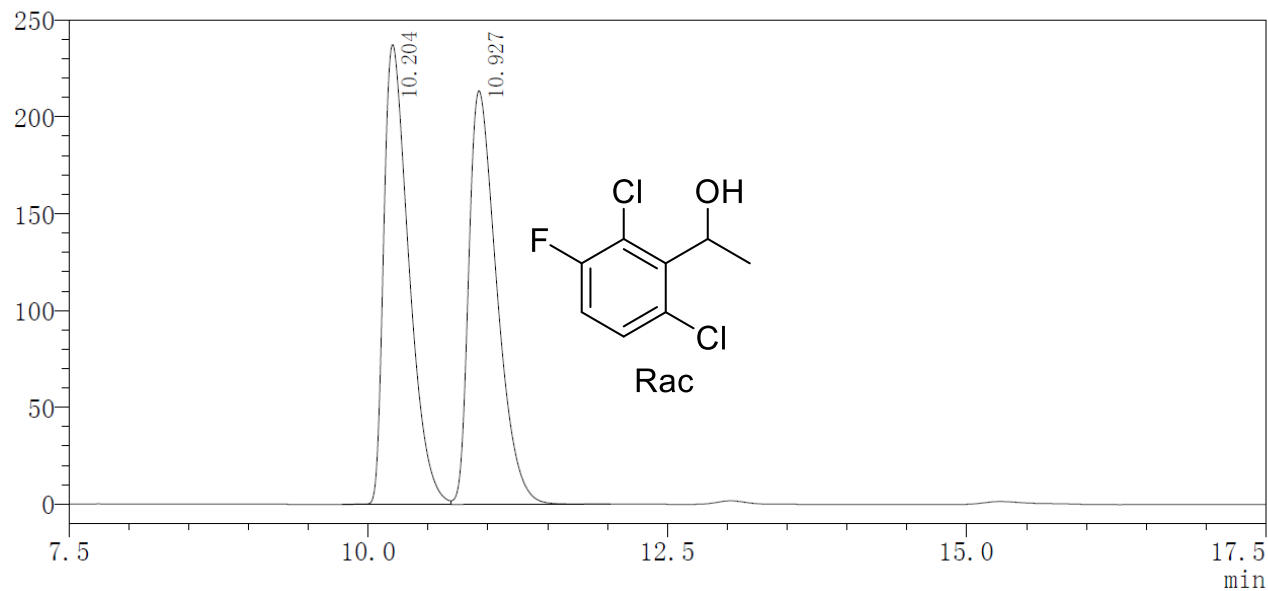

\begin{tabular}{|r|c|l|r|r|r|}
\hline Peak & Ret. Time & Area & Area \% & \multicolumn{1}{|c|}{ Height } & Height \% \\
\hline 1 & 10.204 & 3470210 & 49.911 & 237353 & 52.630 \\
\hline 2 & 10.927 & 3482563 & 50.089 & 213628 & 47.370 \\
\hline
\end{tabular}

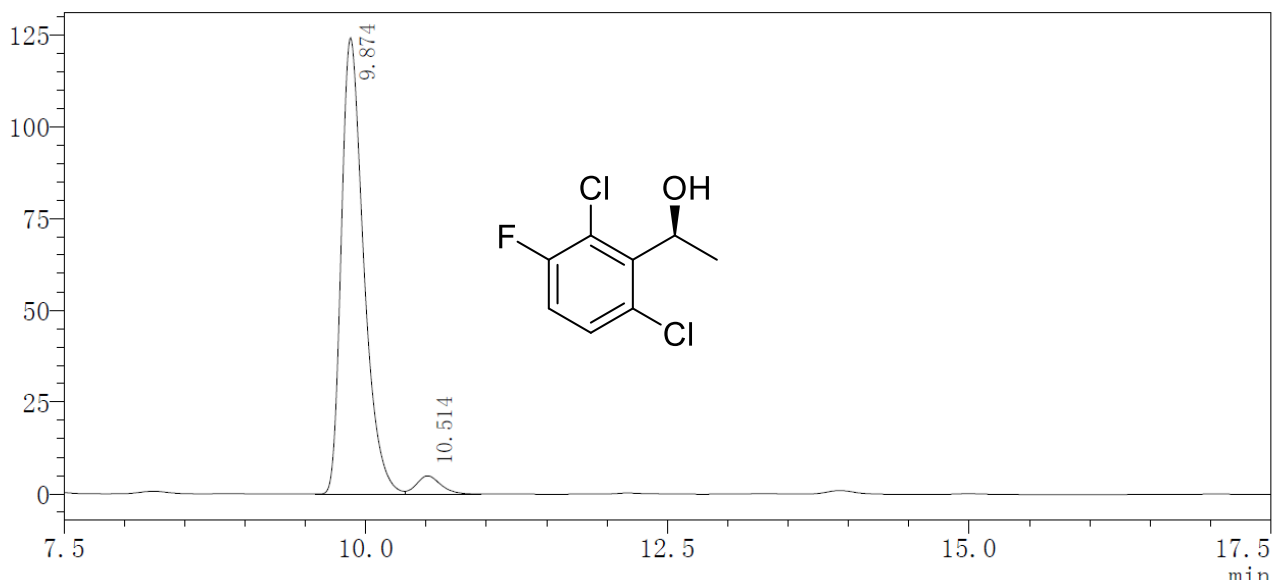

\begin{tabular}{|r|r|r|r|r|r|}
\hline Peak & Ret. Time & \multicolumn{1}{|c|}{ Area } & \multicolumn{1}{|c|}{ Area \% } & Height & \multicolumn{1}{c|}{ Height \% } \\
\hline 1 & 9.874 & 1603776 & 95.866 & 124216 & 96.154 \\
\hline 2 & 10.514 & 69154 & 4.134 & 4969 & 3.846 \\
\hline
\end{tabular}


(S)-1-(2,6-dichlorophenyl)ethan-1-ol (2n)

$\mathrm{mV}$

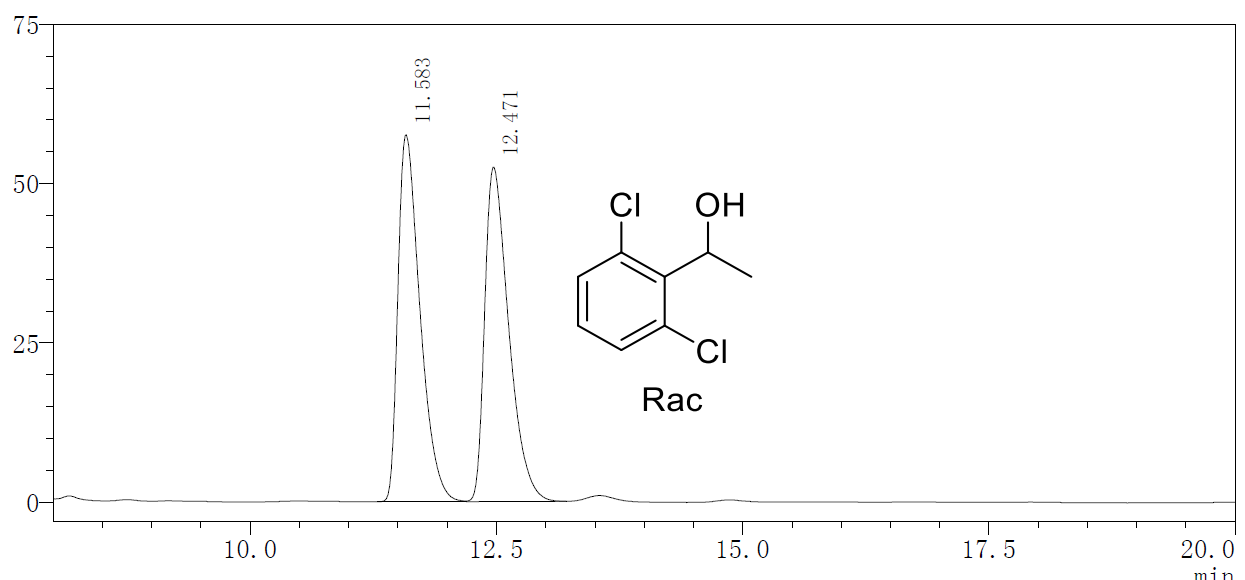

\begin{tabular}{|r|r|r|r|r|r|}
\hline Peak & Ret. Time & Area & Area \% & Height & Height \% \\
\hline 1 & 11.583 & 892139 & 50.051 & 57576 & 52.341 \\
\hline 2 & 12.471 & 890337 & 49.949 & 52425 & 47.659 \\
\hline
\end{tabular}

$\mathrm{mV}$

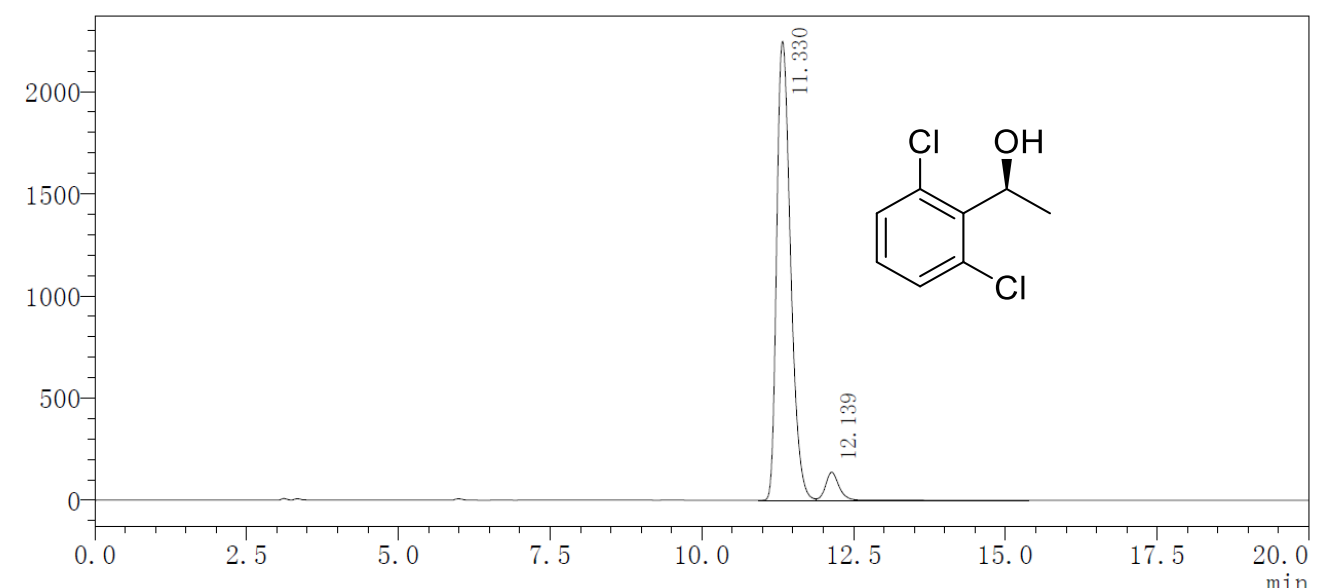

\begin{tabular}{|r|r|r|r|r|r|}
\hline Peak & Ret. Time & \multicolumn{1}{c|}{ Area } & \multicolumn{1}{|c|}{ Area \% } & \multicolumn{1}{c|}{ Height } & Height \% \\
\hline 1 & 11.330 & 35760833 & 94.340 & 2246799 & 94.244 \\
\hline 2 & 12.139 & 2145681 & 5.660 & 137232 & 5.756 \\
\hline
\end{tabular}


(S)-1-(2,6-dimethylphenyl)ethan-1-ol (2o)

$\mathrm{mV}$

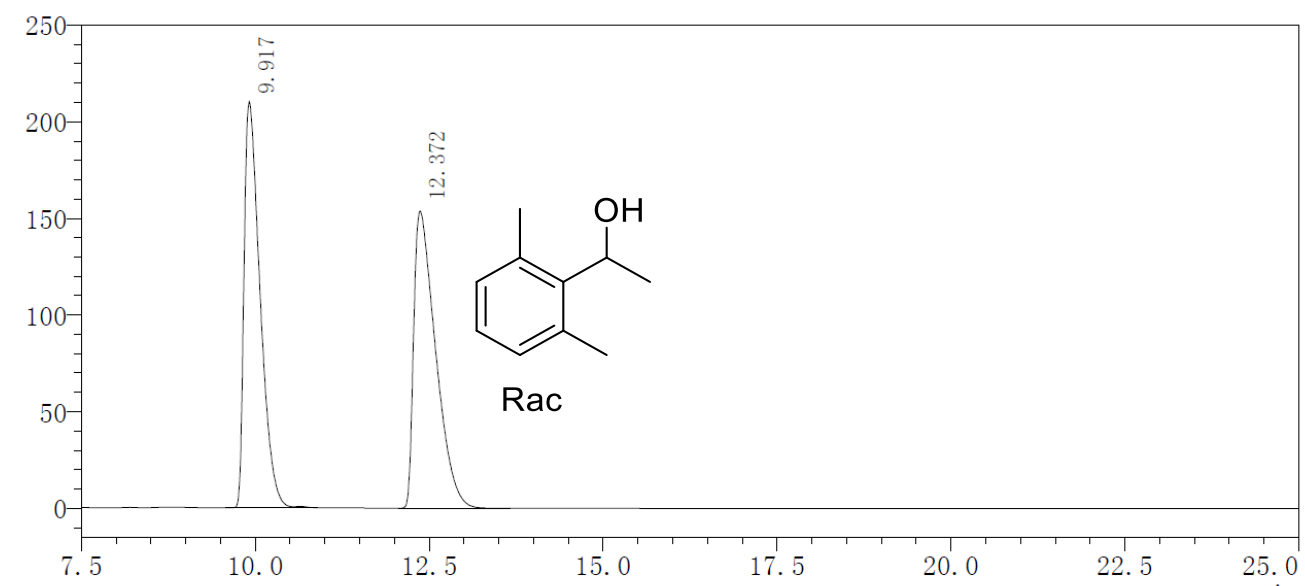

\begin{tabular}{|r|r|r|r|r|r|}
\hline Peak & Ret. Time & Area & Area \% & Height & \multicolumn{1}{c|}{ Height \% } \\
\hline 1 & 9.917 & 3372561 & 50.054 & 210083 & 57.768 \\
\hline 2 & 12.372 & 3365221 & 49.946 & 153586 & 42.232 \\
\hline
\end{tabular}

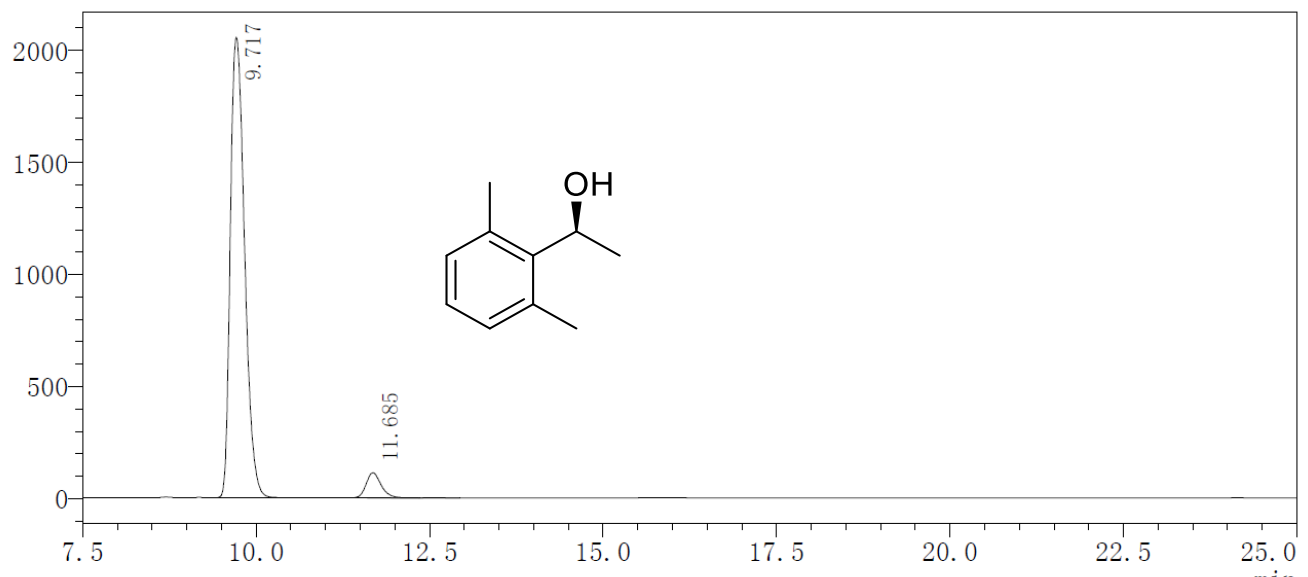

\begin{tabular}{|r|r|r|r|r|r|}
\hline Peak & Ret. Time & \multicolumn{1}{c|}{ Area } & \multicolumn{1}{c|}{ Area \% } & \multicolumn{1}{c|}{ Height } & \multicolumn{1}{c|}{ Height \% } \\
\hline 1 & 9.717 & 29388216 & 94.627 & 2054290 & 94.830 \\
\hline 2 & 11.685 & 1668740 & 5.373 & 112007 & 5.170 \\
\hline
\end{tabular}



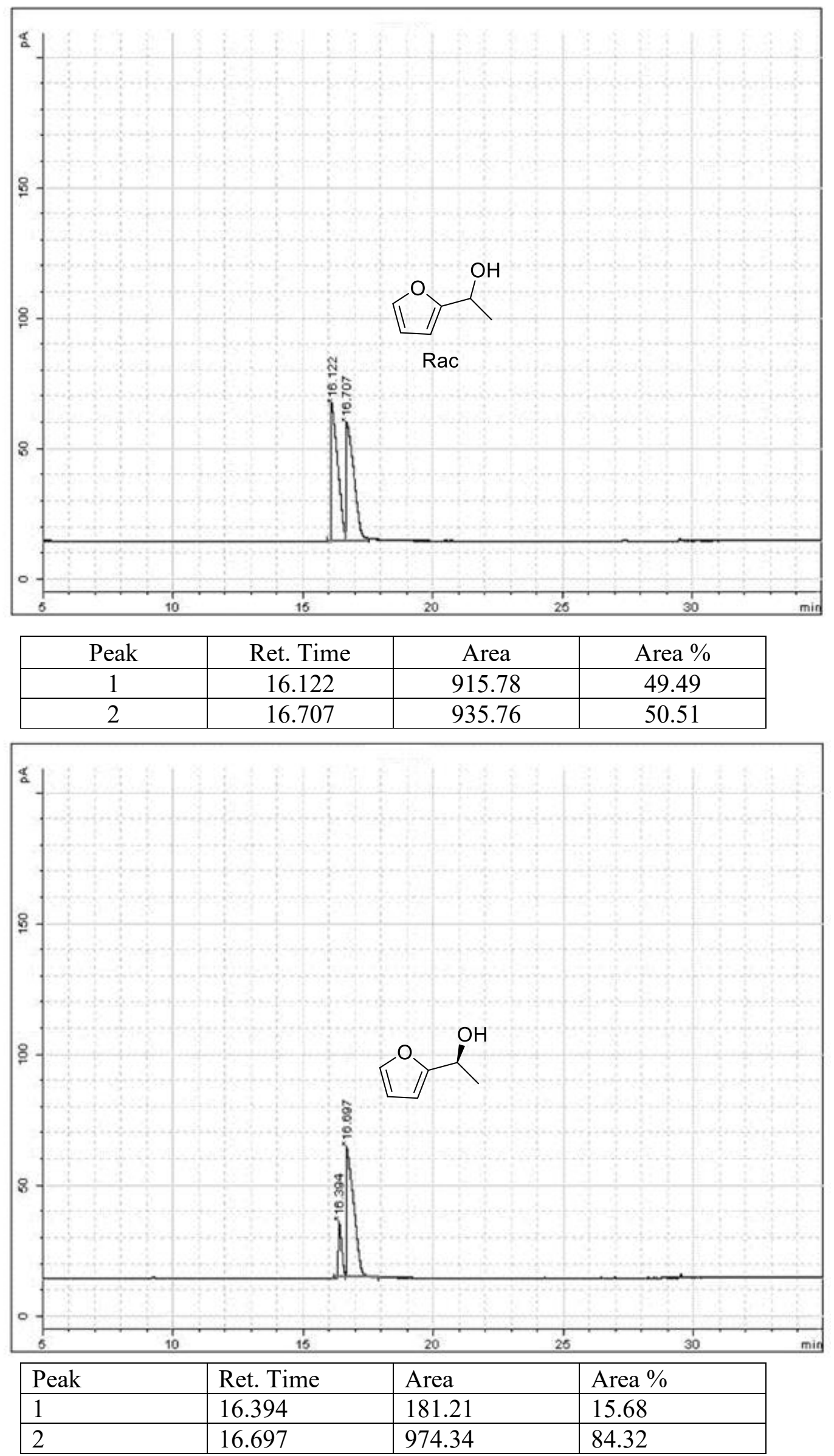

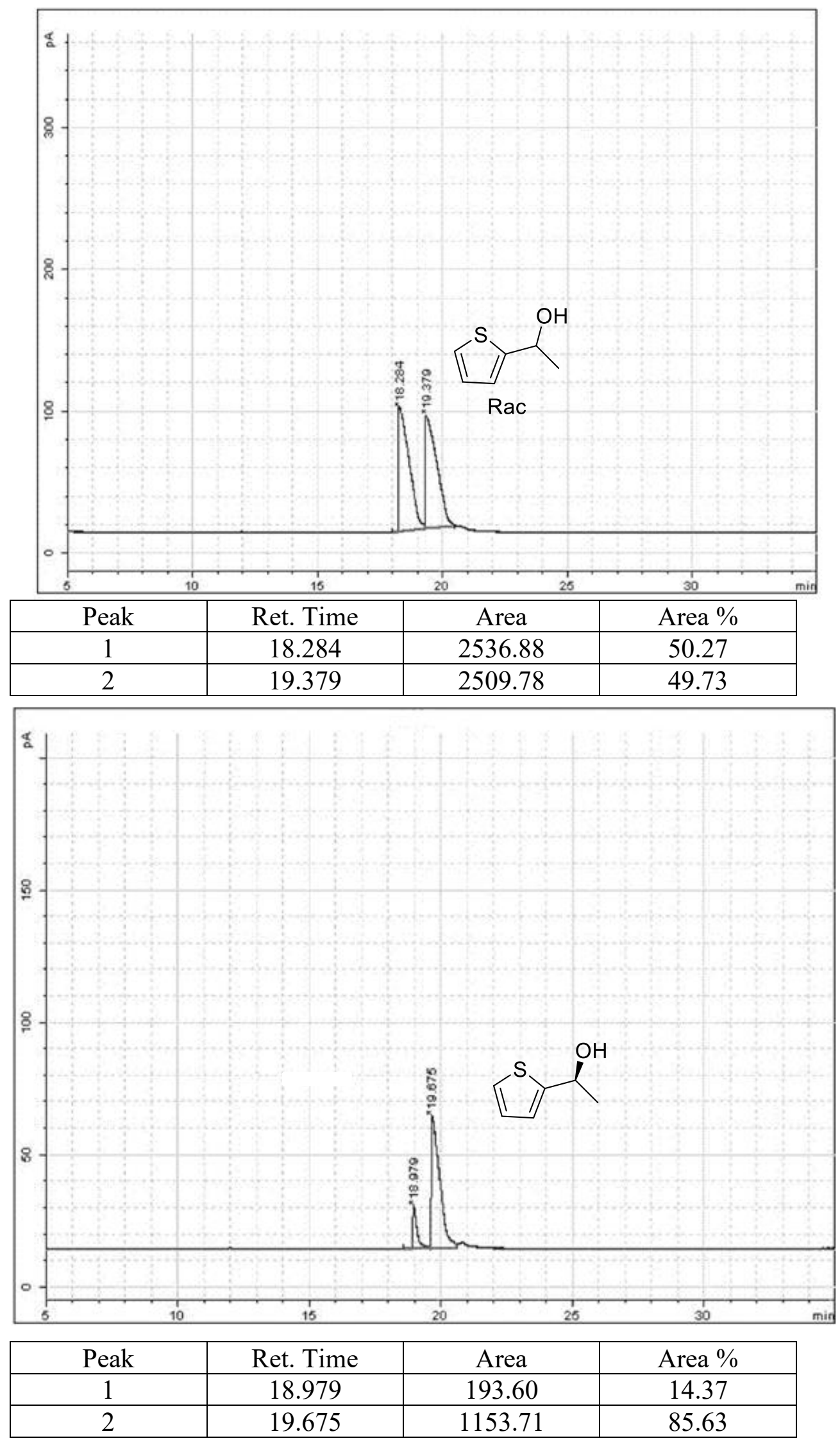
(S)-2,2-dimethyl-1-phenylpropan-1-ol (4a)

$\mathrm{mV}$

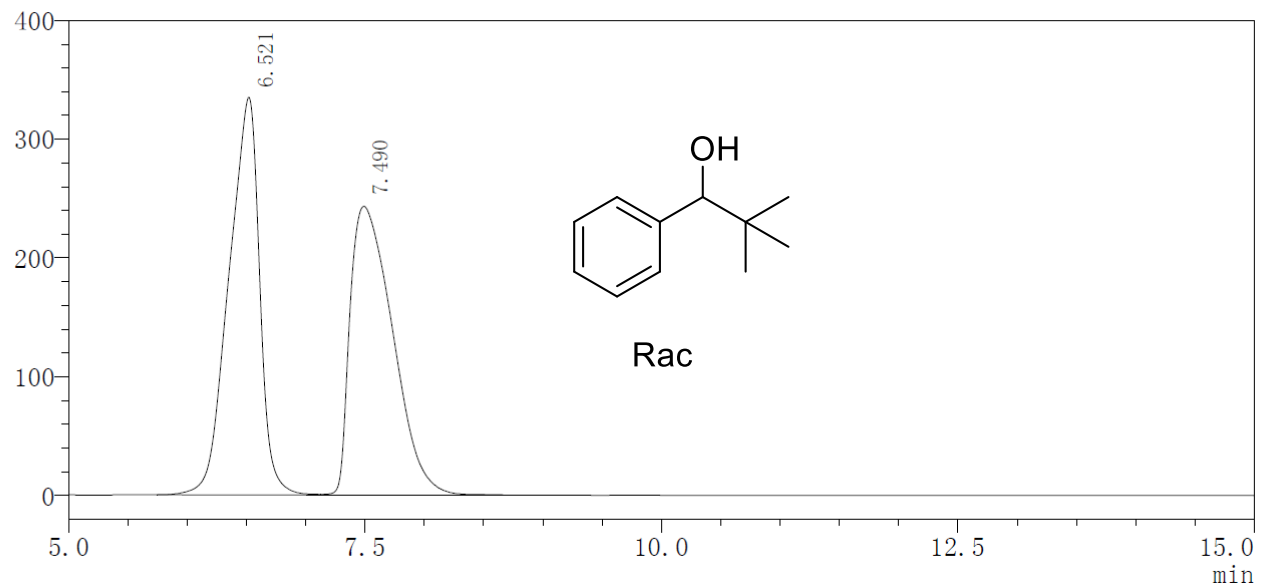

\begin{tabular}{|r|r|r|r|r|r|}
\hline Peak & Ret. Time & Area & Area \% & \multicolumn{1}{|c|}{ Height } & \multicolumn{1}{c|}{ Height \% } \\
\hline 1 & 6.521 & 6042782 & 49.829 & 334703 & 57.913 \\
\hline 2 & 7.490 & 6084321 & 50.171 & 243241 & 42.087 \\
\hline
\end{tabular}

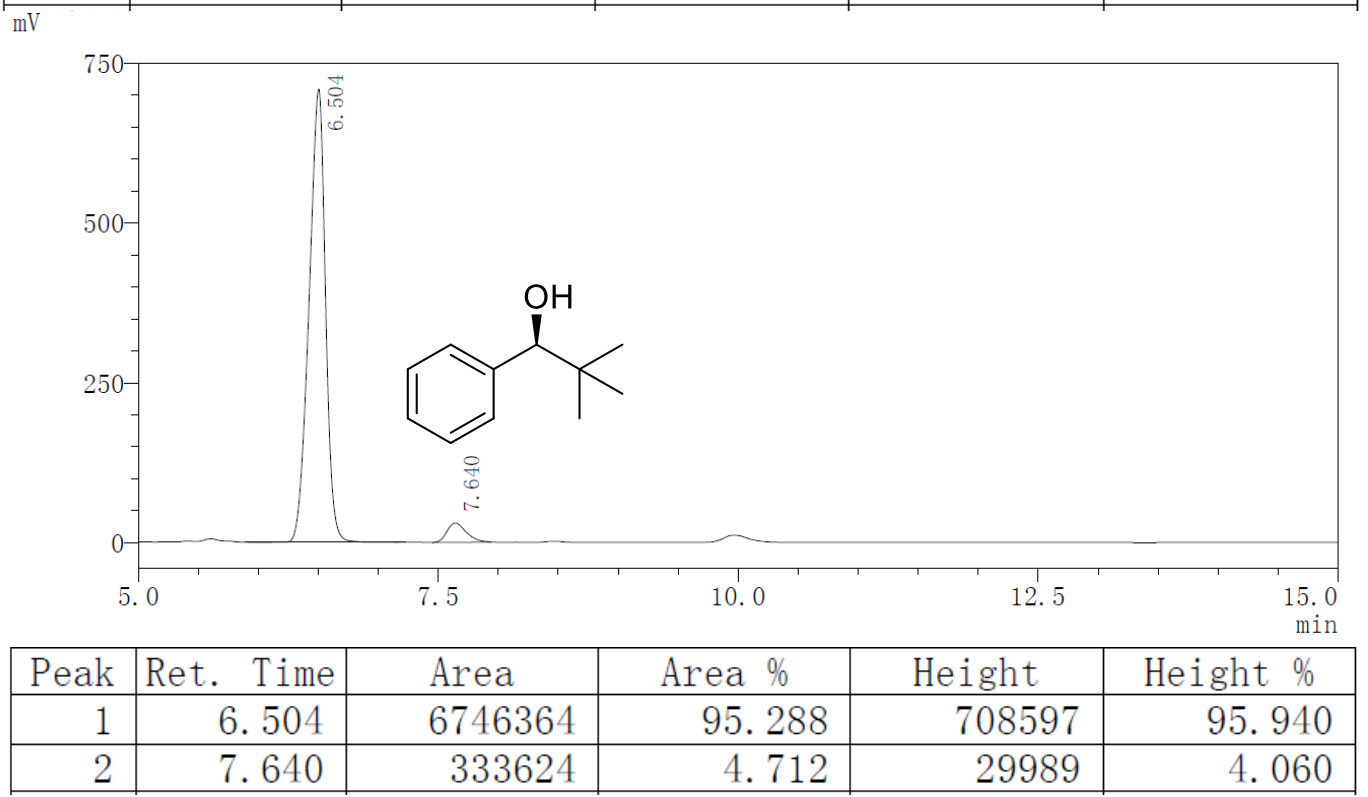


$\mathrm{m} V$

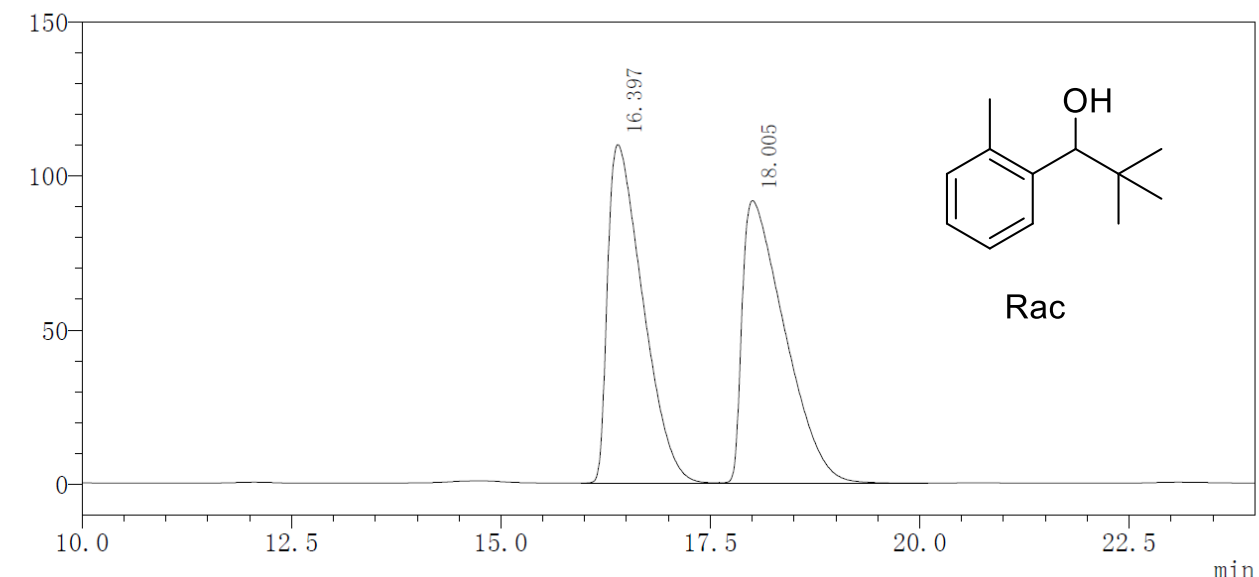

\begin{tabular}{|r|r|r|r|r|r|}
\hline Peak & Ret. Time & Area & Area \% & \multicolumn{1}{c|}{ Height } & Height \% \\
\hline 1 & 16.397 & 3205288 & 49.764 & 109759 & 54.516 \\
\hline 2 & 18.005 & 3235691 & 50.236 & 91573 & 45.484 \\
\hline
\end{tabular}

$\mathrm{mV}$

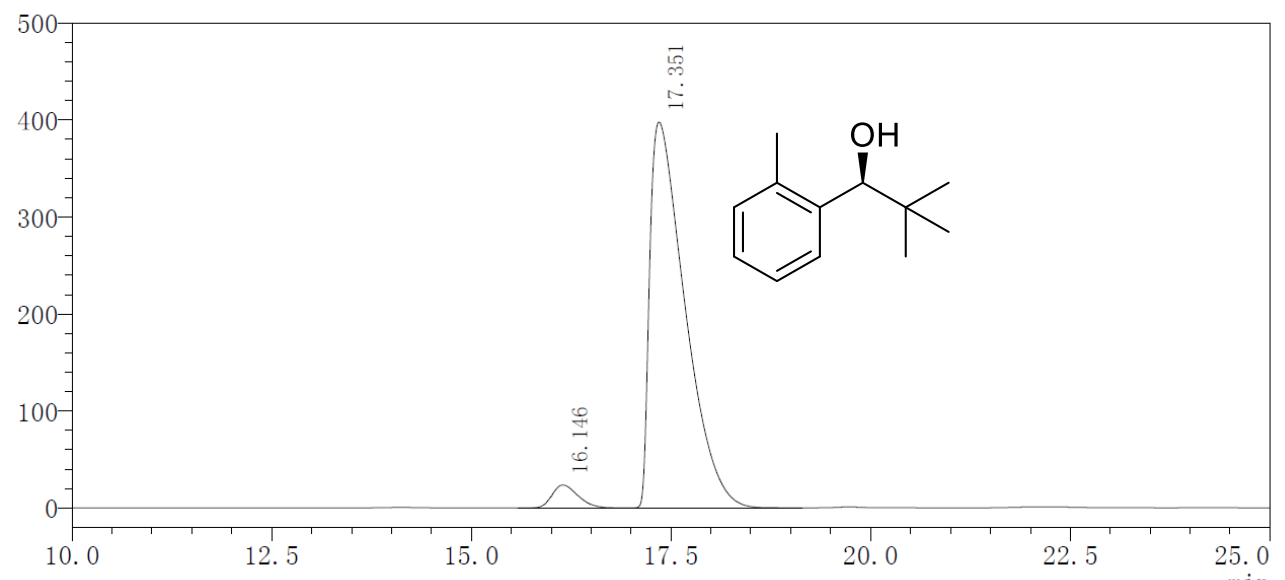

\begin{tabular}{|r|r|r|r|r|r|}
\hline Peak & Ret. Time & \multicolumn{1}{c|}{ Area } & Area \% & \multicolumn{1}{c|}{ Height } & Height \% \\
\hline 1 & 16.146 & 531794 & 4.174 & 23679 & 5.616 \\
\hline 2 & 17.351 & 12210134 & 95.826 & 397957 & 94.384 \\
\hline
\end{tabular}


(S)-1-(4-methoxyphenyl)-2,2-dimethylpropan-1-ol (4c)

mV

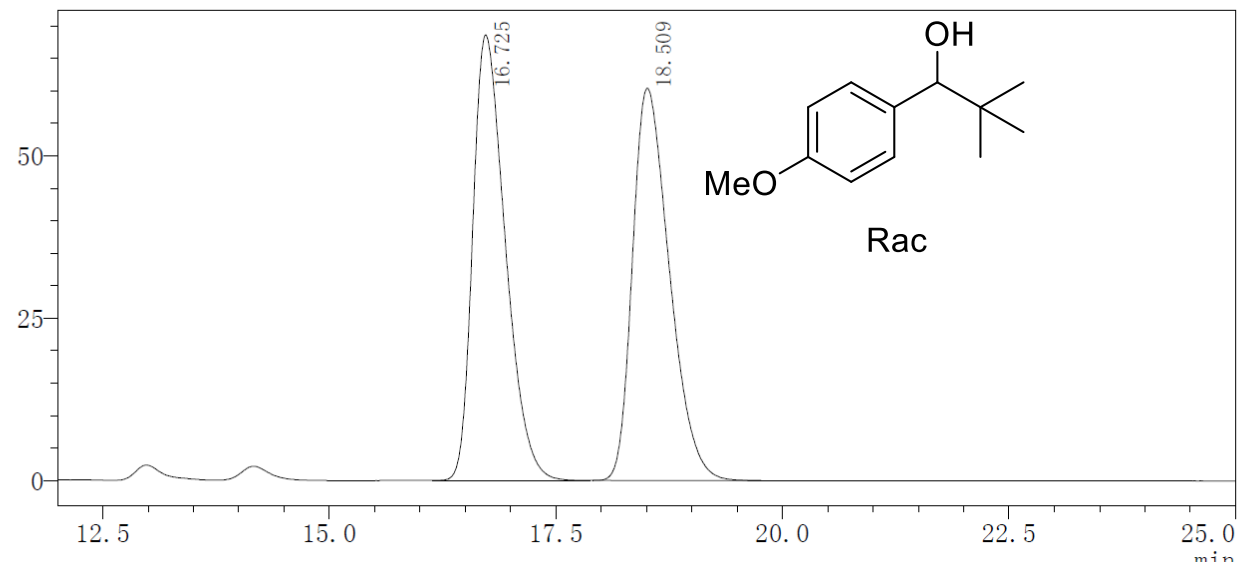

\begin{tabular}{|r|r|r|r|r|r|}
\hline Peak & Ret. Time & Area & Area \% & Height & \multicolumn{1}{|c|}{ Height \% } \\
\hline 1 & 16.725 & 1717601 & 50.037 & 68603 & 53.171 \\
\hline 2 & 18.509 & 1715069 & 49.963 & 60419 & 46.829 \\
\hline
\end{tabular}

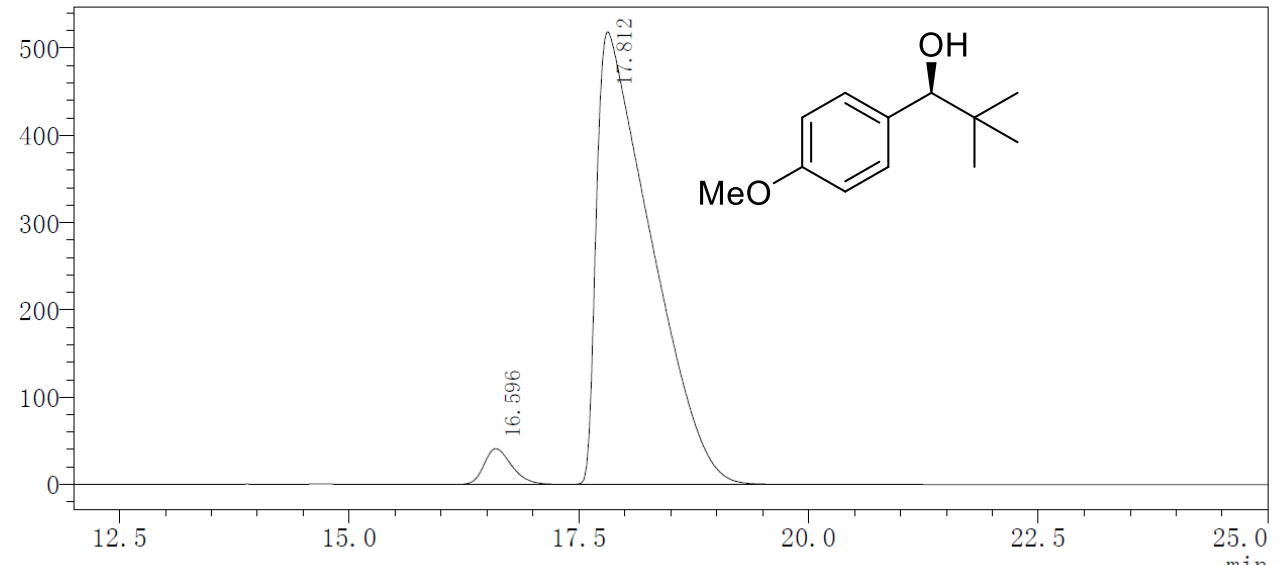

\begin{tabular}{|r|r|r|r|r|r|}
\hline Peak & Ret. Time & \multicolumn{1}{c|}{ Area } & \multicolumn{1}{|c|}{ Area \% } & Height & Height \% \\
\hline 1 & 16.596 & 861484 & 3.871 & 40949 & 7.322 \\
\hline 2 & 17.812 & 21391472 & 96.129 & 518322 & 92.678 \\
\hline
\end{tabular}


(S)-1-(2-bromophenyl)-2,2-dimethylpropan-1-ol(4d)

$\mathrm{mV}$

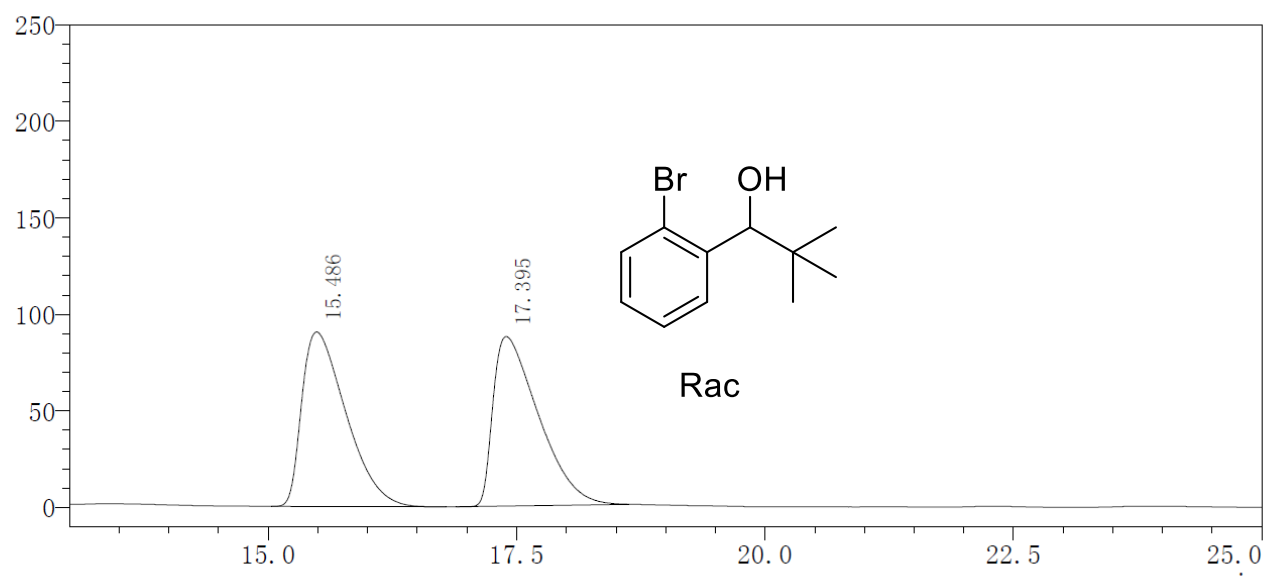

\begin{tabular}{|r|r|r|r|r|r|}
\hline Peak & Ret. Time & Area & Area \% & Height & Height \% \\
\hline 1 & 15.486 & 2788822 & 49.955 & 90443 & 50.726 \\
\hline 2 & 17.395 & 2793826 & 50.045 & 87853 & 49.274 \\
\hline
\end{tabular}

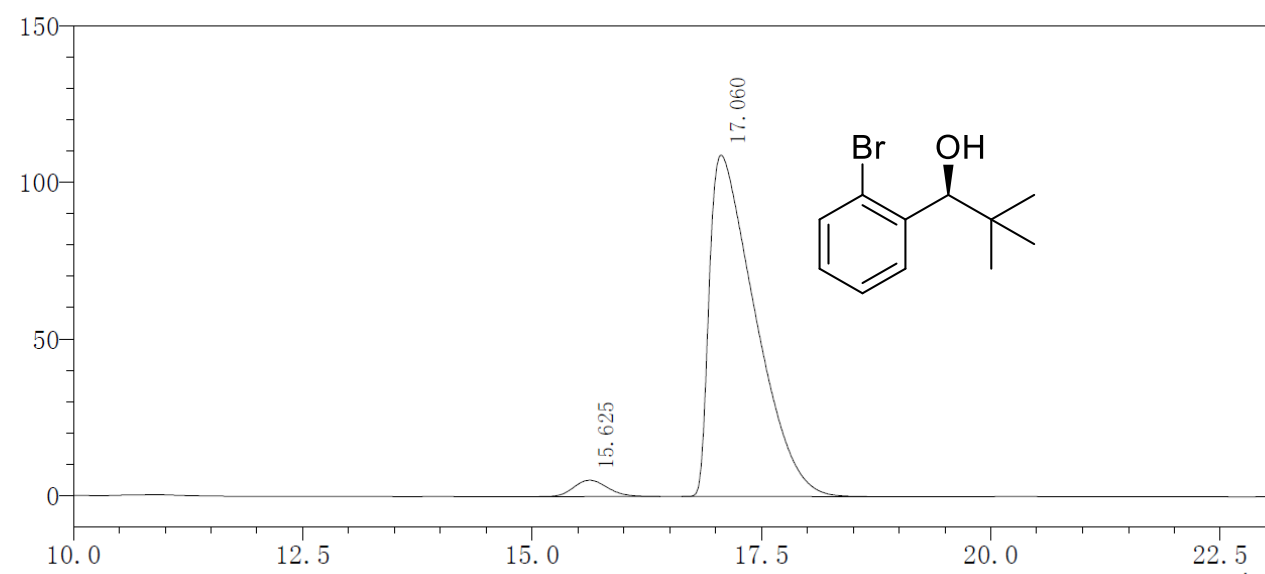

\begin{tabular}{|r|r|r|r|r|r|}
\hline Peak & Ret. Time & \multicolumn{1}{c|}{ Area } & Area \% & Height & Height \% \\
\hline 1 & 15.625 & 132920 & 3.390 & 5191 & 4.545 \\
\hline 2 & 17.060 & 3787914 & 96.610 & 109017 & 95.455 \\
\hline
\end{tabular}


(S)-1-(3-bromophenyl)-2,2-dimethylpropan-1-ol (4e)

$\mathrm{mV}$

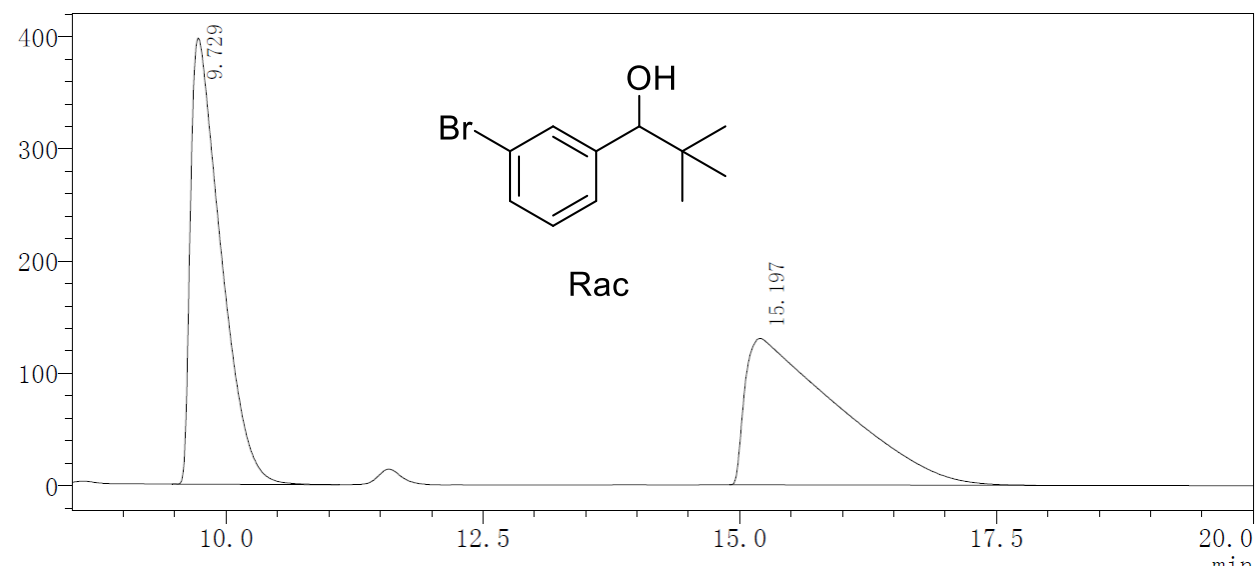

\begin{tabular}{|r|r|r|r|r|r|}
\hline Peak & Ret. Time & Area & Area \% & Height & \multicolumn{1}{|c|}{ Height \% } \\
\hline 1 & 9.729 & 8207390 & 50.055 & 397288 & 75.288 \\
\hline 2 & 15.197 & 8189317 & 49.945 & 130404 & 24.712 \\
\hline
\end{tabular}

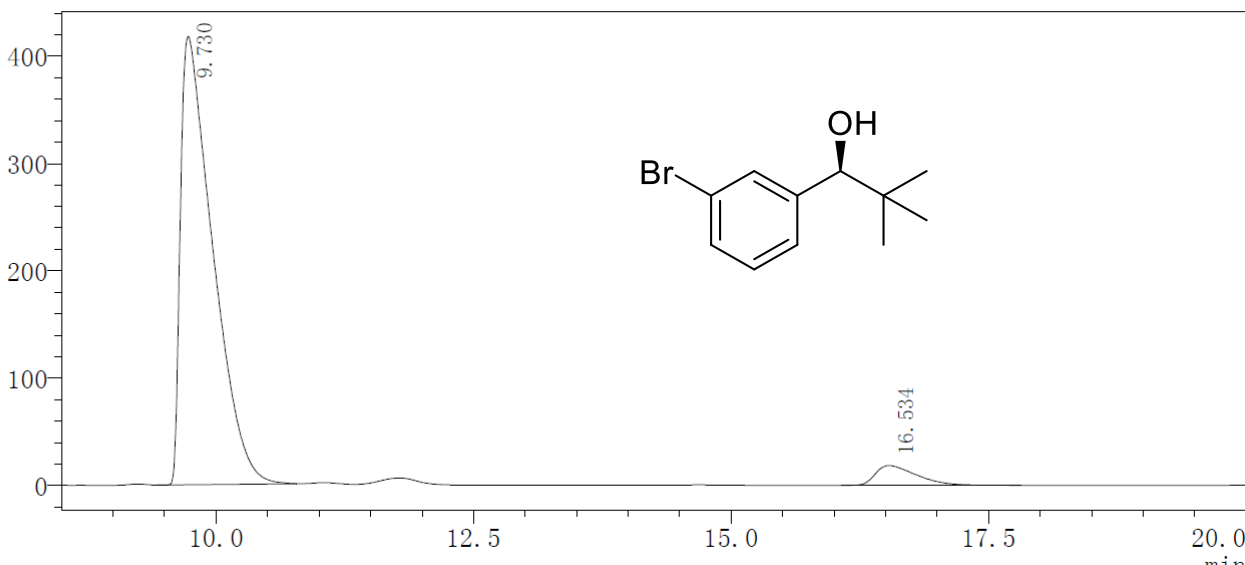

\begin{tabular}{|r|r|r|r|r|r|}
\hline Peak & Ret. Time & \multicolumn{1}{|c|}{ Area } & Area \% & \multicolumn{1}{c|}{ Height } & Height \% \\
\hline 1 & 9.730 & 9088281 & 94.729 & 417501 & 95.778 \\
\hline 2 & 16.534 & 505694 & 5.271 & 18403 & 4.222 \\
\hline
\end{tabular}


(S)-1-(4-bromophenyl)-2,2-dimethylpropan-1-ol (4f)

$\mathrm{mV}$

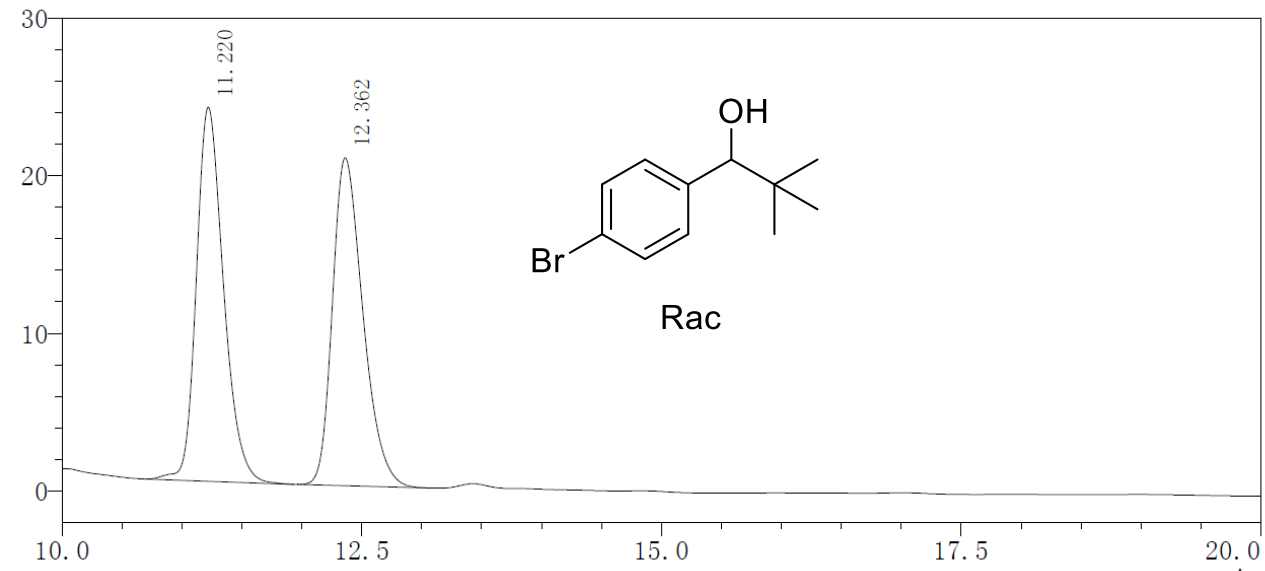

\begin{tabular}{|r|r|r|r|r|r|}
\hline Peak & Ret. Time & \multicolumn{1}{c|}{ Area } & Area \% & Height & Height \% \\
\hline 1 & 11.220 & 375384 & 50.451 & 23732 & 53.289 \\
\hline 2 & 12.362 & 368671 & 49.549 & 20803 & 46.711 \\
\hline
\end{tabular}

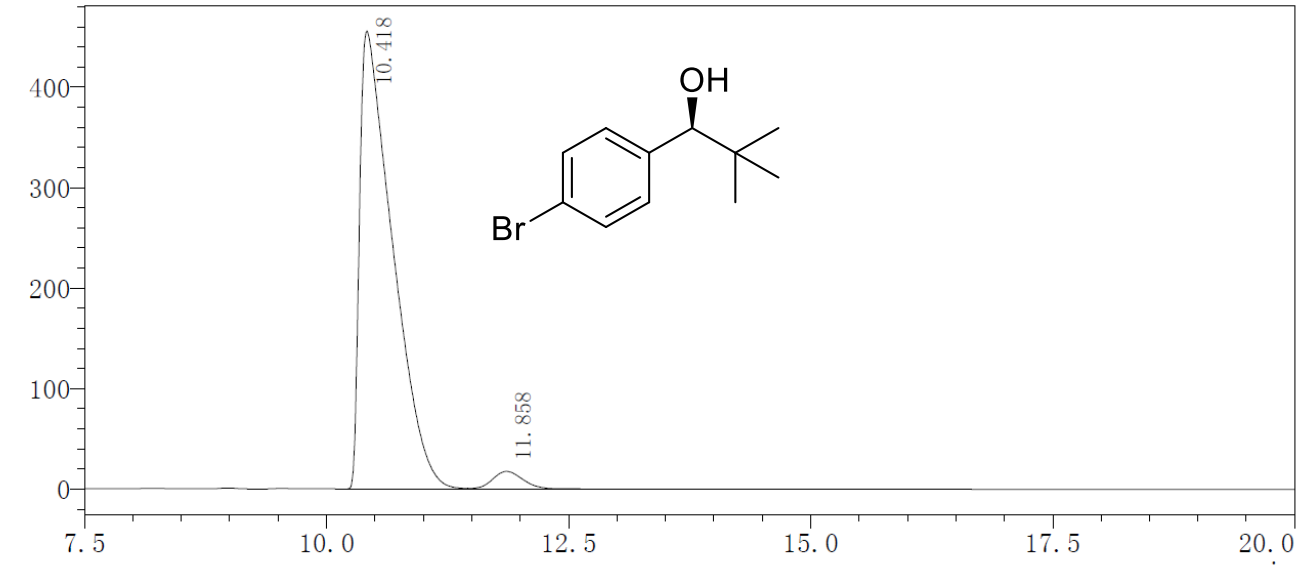

\begin{tabular}{|r|r|r|r|r|r|}
\hline Peak & Ret. Time & \multicolumn{1}{c|}{ Area } & \multicolumn{1}{c|}{ Area \% } & Height & Height \% \\
\hline 1 & 10.418 & 10790394 & 96.457 & 455941 & 96.274 \\
\hline 2 & 11.858 & 396348 & 3.543 & 17648 & 3.726 \\
\hline
\end{tabular}




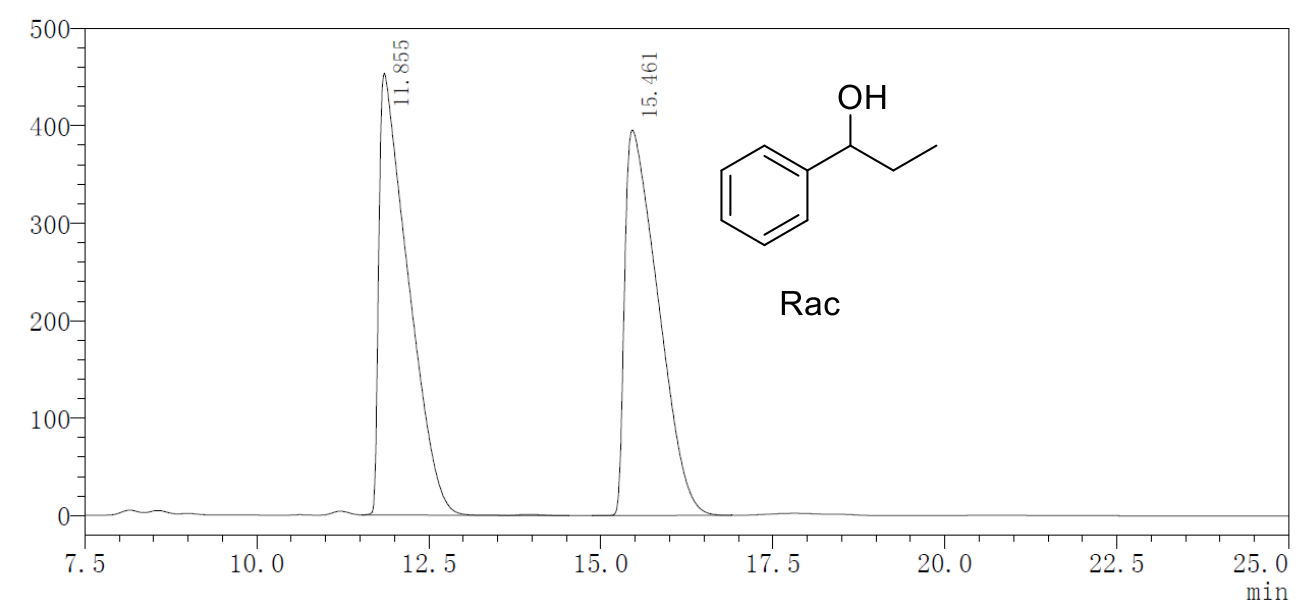

\begin{tabular}{|r|r|r|r|r|r|}
\hline Peak & Ret. Time & Area & \multicolumn{1}{|c|}{ Area \% } & Height & Height \% \\
\hline 1 & 11.855 & 13394945 & 50.080 & 453125 & 53.393 \\
\hline 2 & 15.461 & 13352109 & 49.920 & 395537 & 46.607 \\
\hline
\end{tabular}

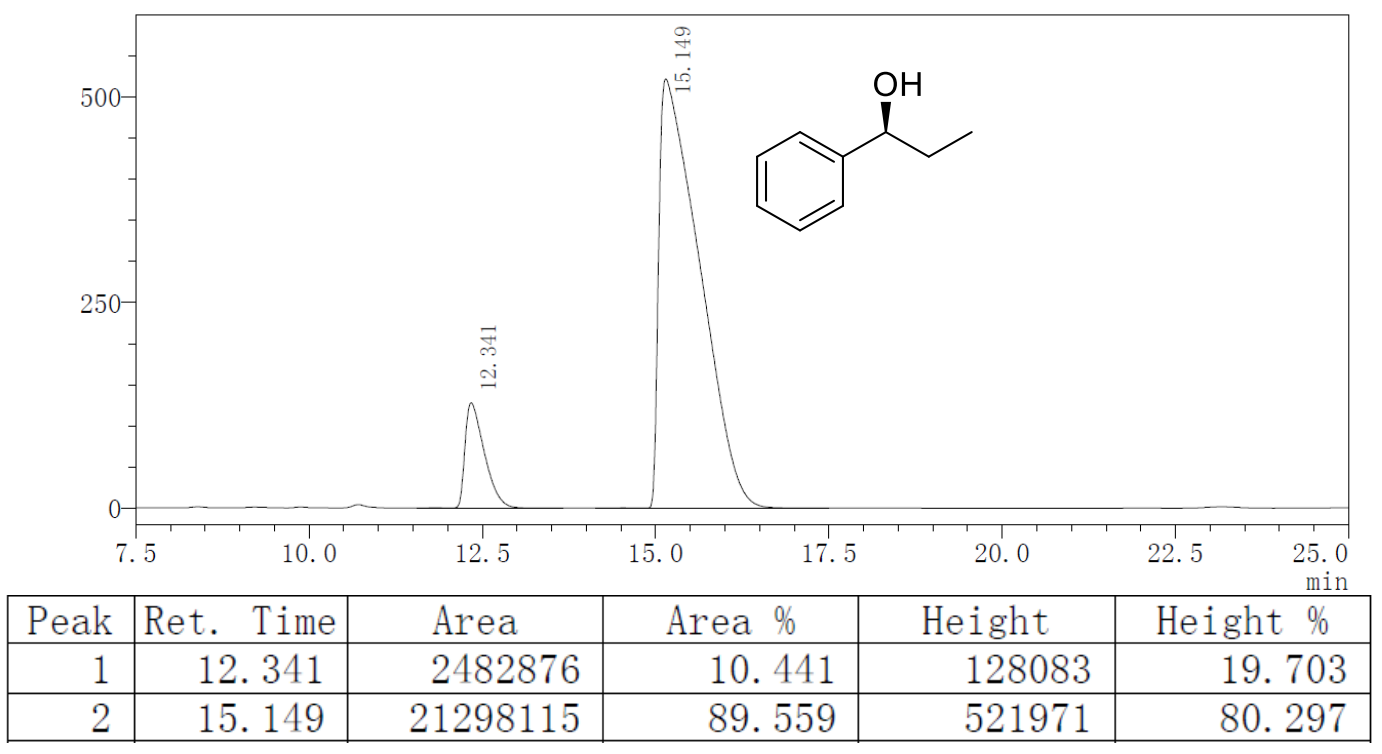




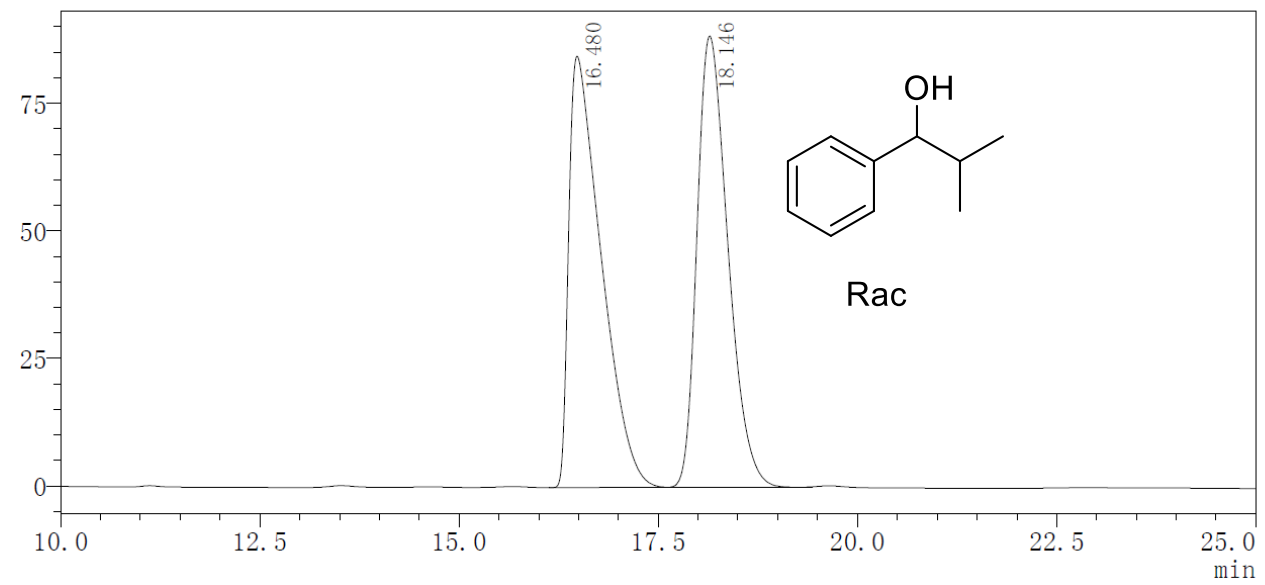

\begin{tabular}{|r|c|c|r|r|r|}
\hline Peak & Ret. Time & Area & Area \% & Height & Height \% \\
\hline 1 & 16.480 & 2393066 & 50.094 & 84537 & 48.895 \\
\hline 2 & 18.146 & 2384112 & 49.906 & 88356 & 51.105 \\
\hline
\end{tabular}

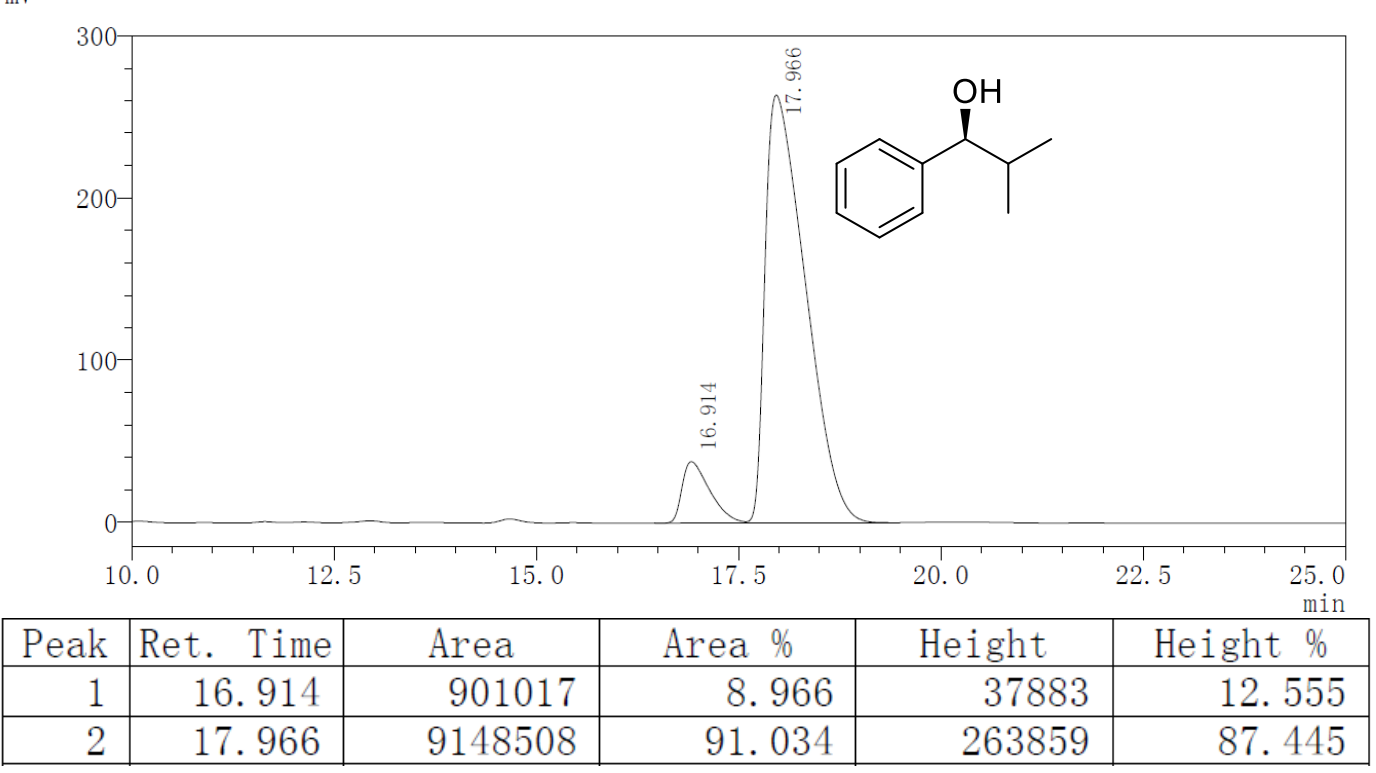


$\mathrm{mV}$

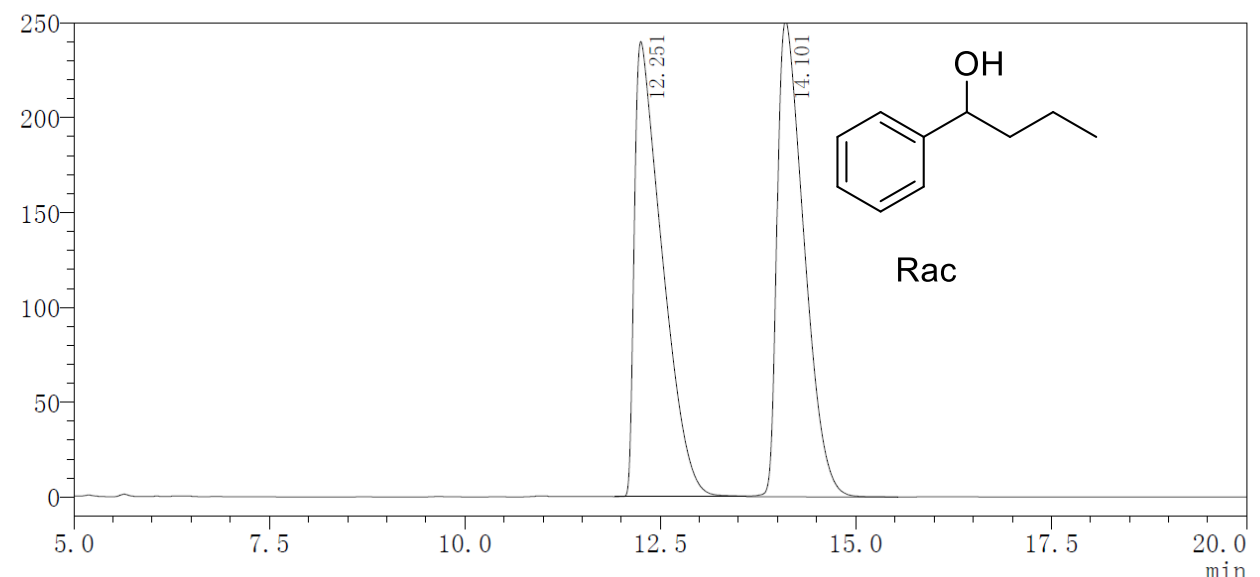

\begin{tabular}{|r|r|r|r|r|r|}
\hline Peak & Ret. Time & Area & \multicolumn{1}{|c|}{ Area \% } & Height & Height \% \\
\hline 1 & 12.251 & 5944790 & 49.866 & 239937 & 48.883 \\
\hline 2 & 14.101 & 5976705 & 50.134 & 250897 & 51.117 \\
\hline
\end{tabular}

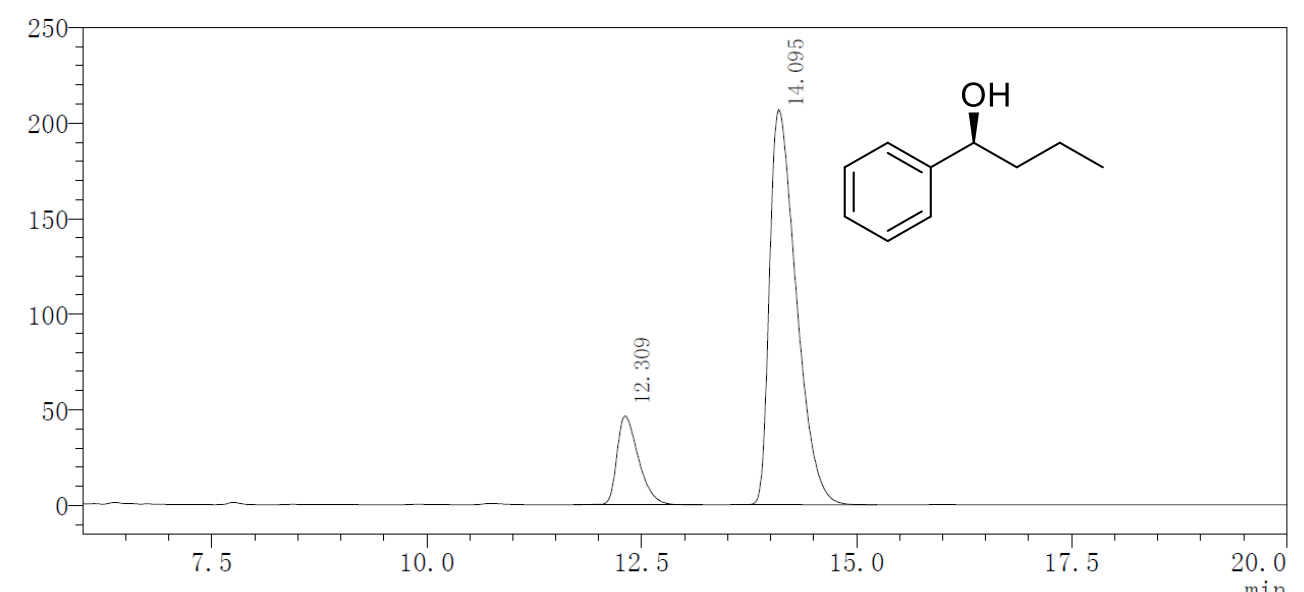

\begin{tabular}{|r|r|r|r|r|r|}
\hline Peak & Ret. Time & \multicolumn{1}{|c|}{ Area } & \multicolumn{1}{|c|}{ Area \% } & Height & \multicolumn{1}{c|}{ Height \% } \\
\hline 1 & 12.309 & 809079 & 15.358 & 46415 & 18.360 \\
\hline 2 & 14.095 & 4458913 & 84.642 & 206383 & 81.640 \\
\hline
\end{tabular}


(S)-cyclopentyl(phenyl)methanol (4j)

$\mathrm{mV}$

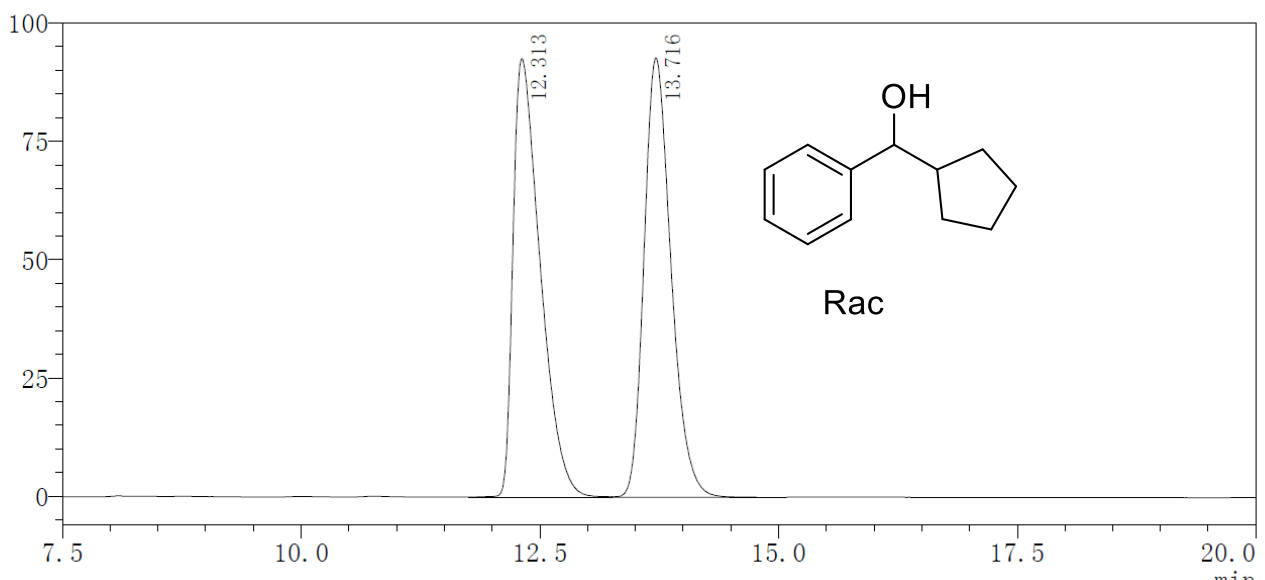

\begin{tabular}{|r|r|r|r|r|r|}
\hline Peak & Ret. Time & Area & Area \% & \multicolumn{1}{|c|}{ Height } & Height \% \\
\hline 1 & 12.313 & 1833246 & 50.071 & 92578 & 49.941 \\
\hline 2 & 13.716 & 1828052 & 49.929 & 92796 & 50.059 \\
\hline
\end{tabular}

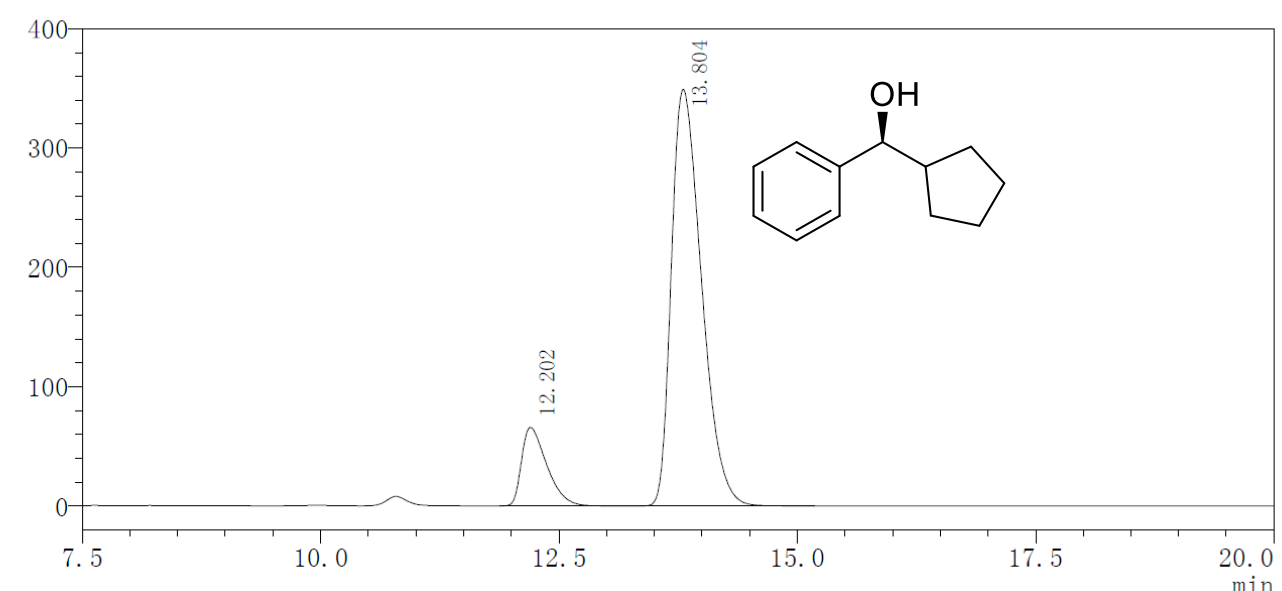

\begin{tabular}{|r|r|r|r|r|r|}
\hline Peak & Ret. Time & \multicolumn{1}{|c|}{ Area } & \multicolumn{1}{|c|}{ Area \% } & Height & Height \% \\
\hline 1 & 12.202 & 1207004 & 13.574 & 65763 & 15.854 \\
\hline 2 & 13.804 & 7684838 & 86.426 & 349049 & 84.146 \\
\hline
\end{tabular}




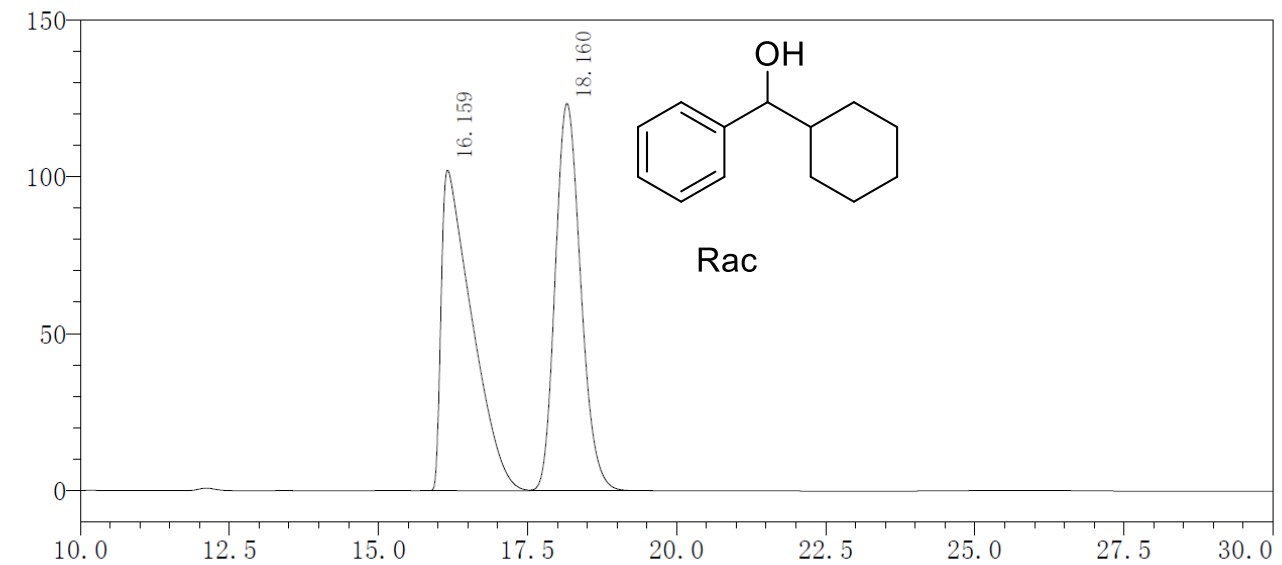

\begin{tabular}{|r|r|r|r|r|r|}
\hline Peak & Ret. Time & Area & \multicolumn{1}{|c|}{ Area \% } & Height & Height \% \\
\hline 1 & 16.159 & 3660851 & 50.017 & 102226 & 45.293 \\
\hline 2 & 18.160 & 3658433 & 49.983 & 123472 & 54.707 \\
\hline
\end{tabular}

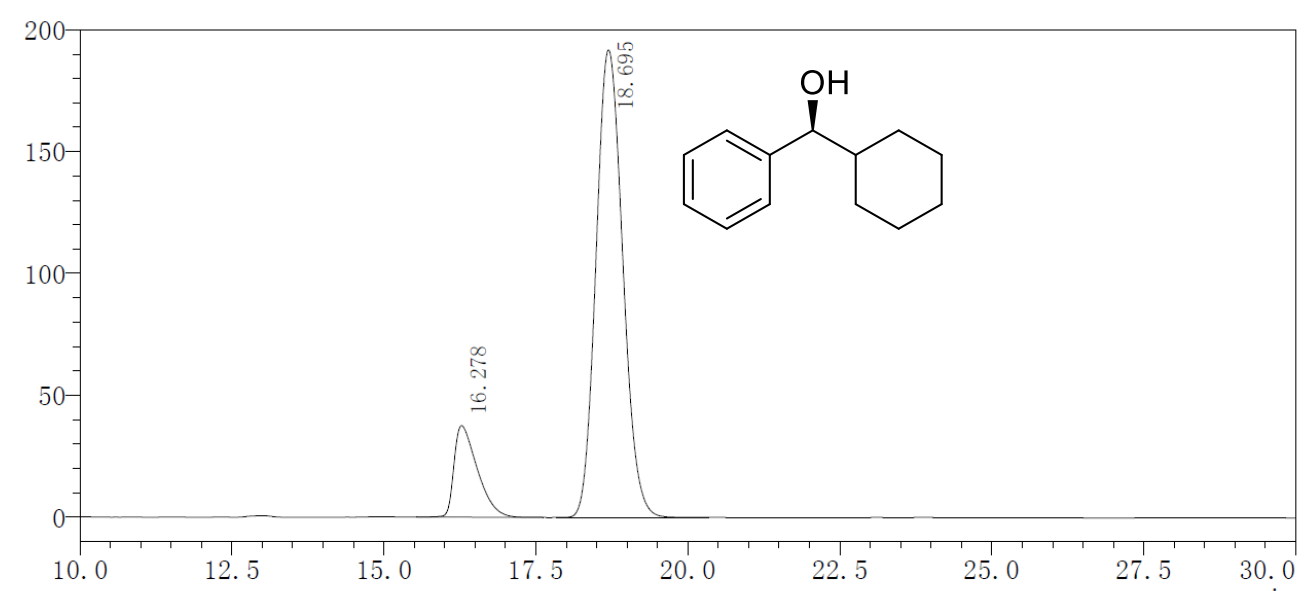

\begin{tabular}{|r|r|r|r|r|r|}
\hline Peak & Ret. Time & Area & \multicolumn{1}{c|}{ Area \% } & Height & Height \% \\
\hline 1 & 16.278 & 1005752 & 14.597 & 37603 & 16.381 \\
\hline 2 & 18.695 & 5884333 & 85.403 & 191956 & 83.619 \\
\hline
\end{tabular}




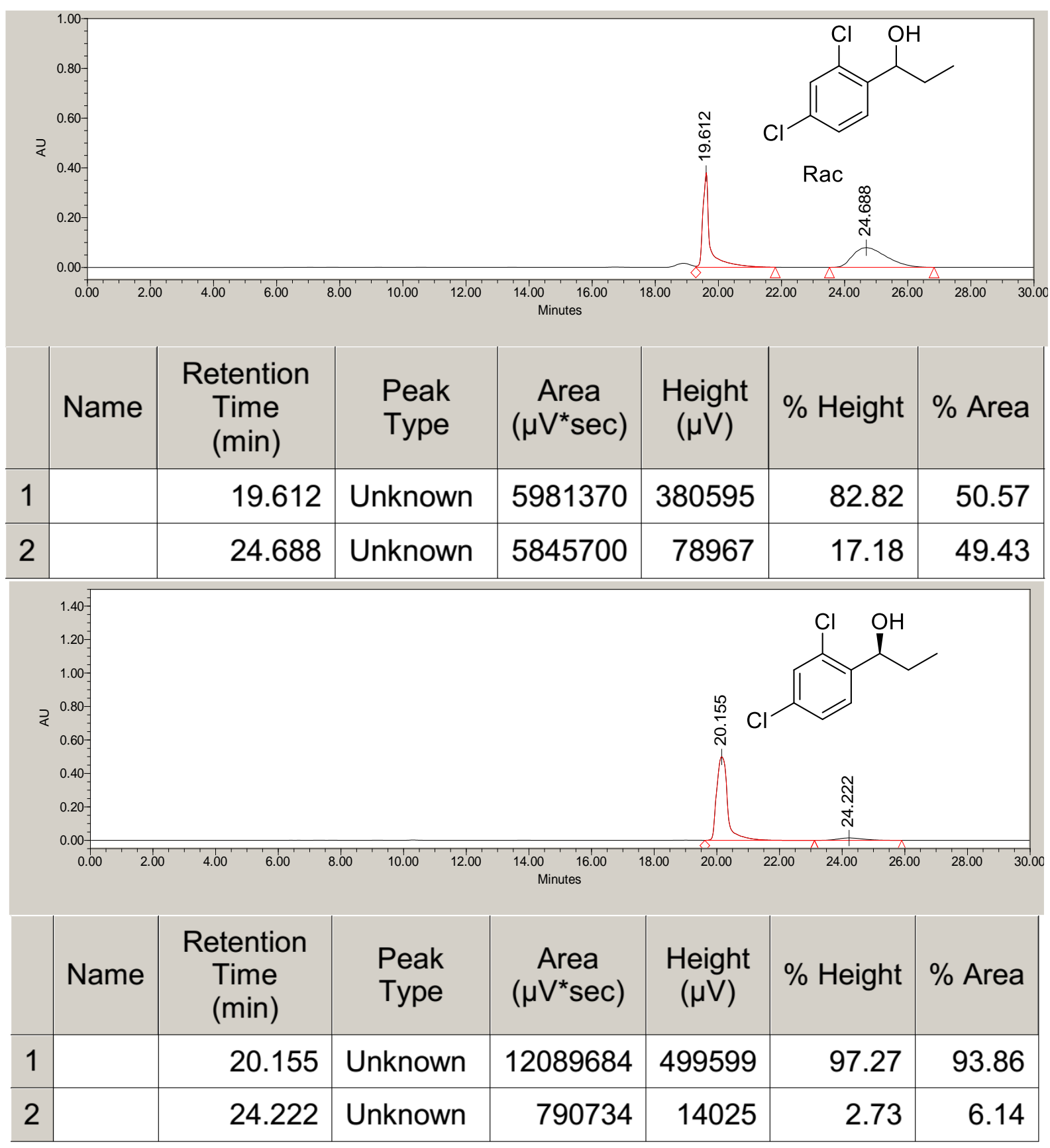


(S)-1-(naphthalen-1-yl)ethan-1-ol (4m)

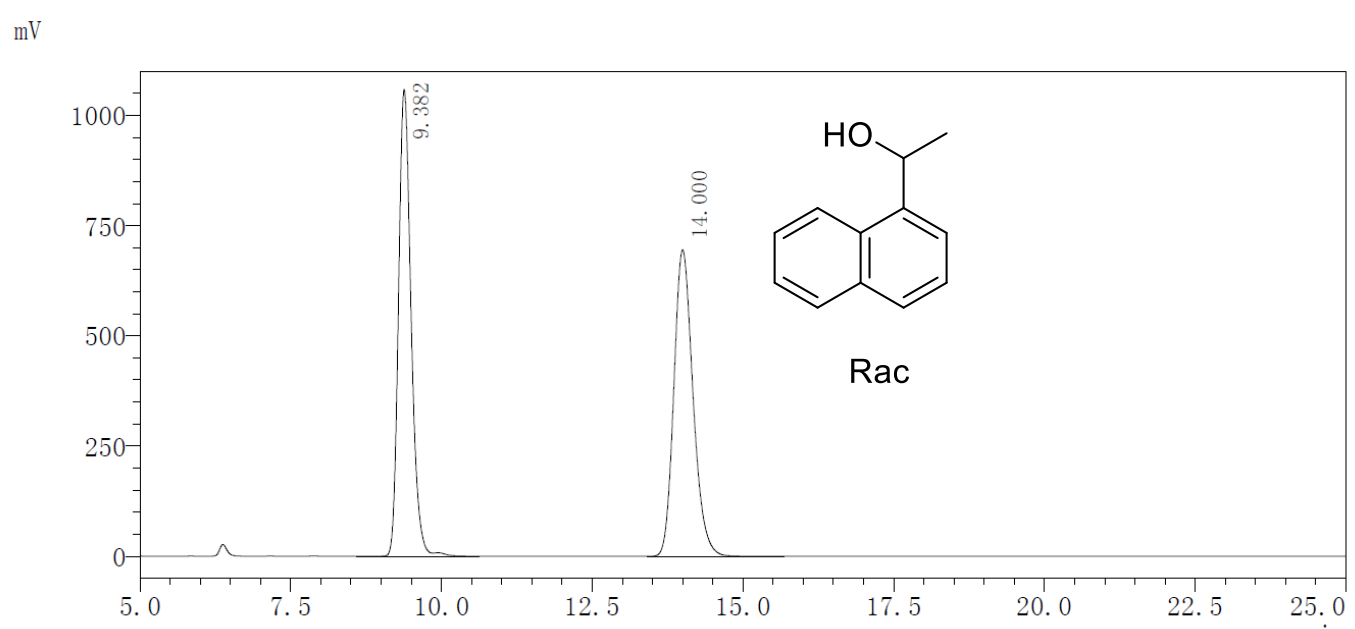

\begin{tabular}{|r|r|r|r|r|r|}
\hline Peak & Ret. Time & \multicolumn{1}{c|}{ Area } & \multicolumn{1}{c|}{ Area \% } & Height & Height \% \\
\hline 1 & 9.382 & 15380083 & 49.999 & 1059250 & 60.370 \\
\hline 2 & 14.000 & 15380988 & 50.001 & 695356 & 39.630 \\
\hline
\end{tabular}

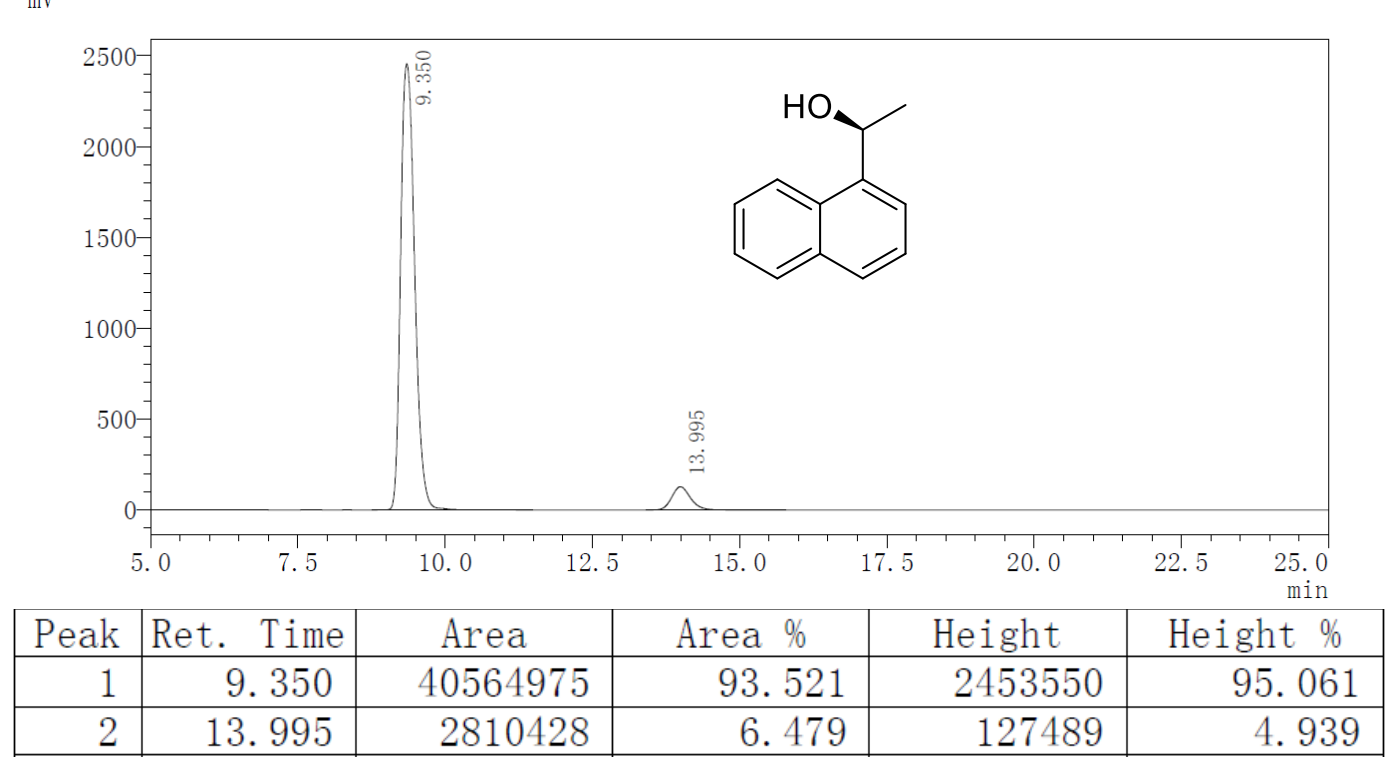


(S)-1-(anthracen-9-yl)ethan-1-ol (4n)

$\mathrm{mV}$

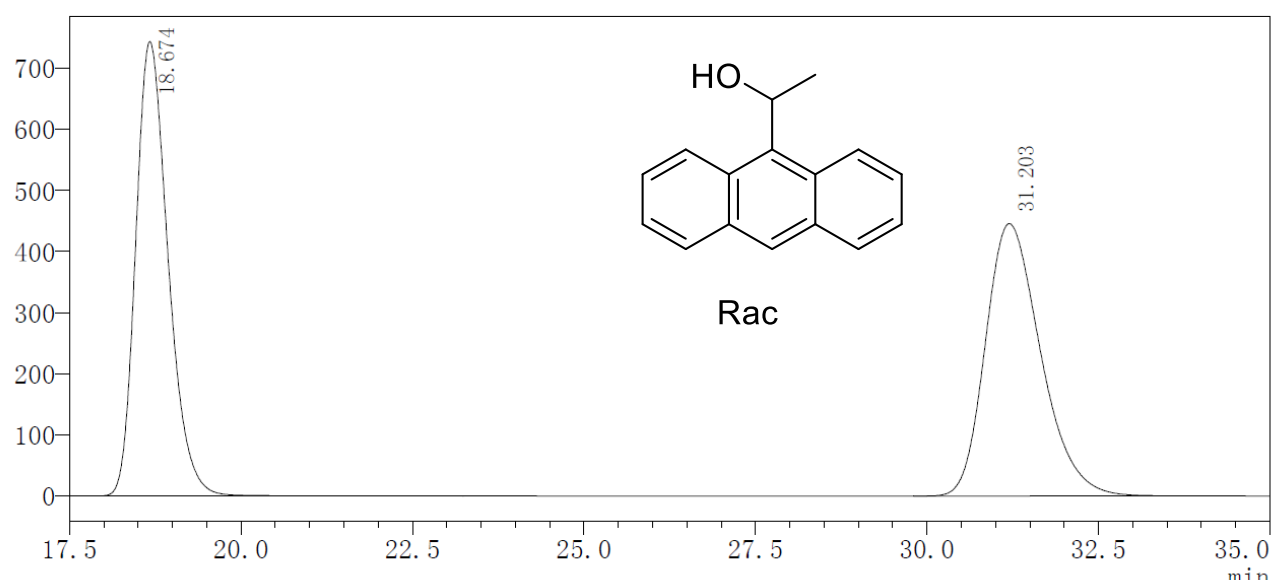

\begin{tabular}{|r|r|r|r|r|r|}
\hline Peak & Ret. Time & Area & Area \% & Height & Height \% \\
\hline 1 & 18.674 & 24390029 & 49.450 & 743466 & 62.522 \\
\hline 2 & 31.203 & 24932780 & 50.550 & 445659 & 37.478 \\
\hline
\end{tabular}

$\mathrm{mV}$

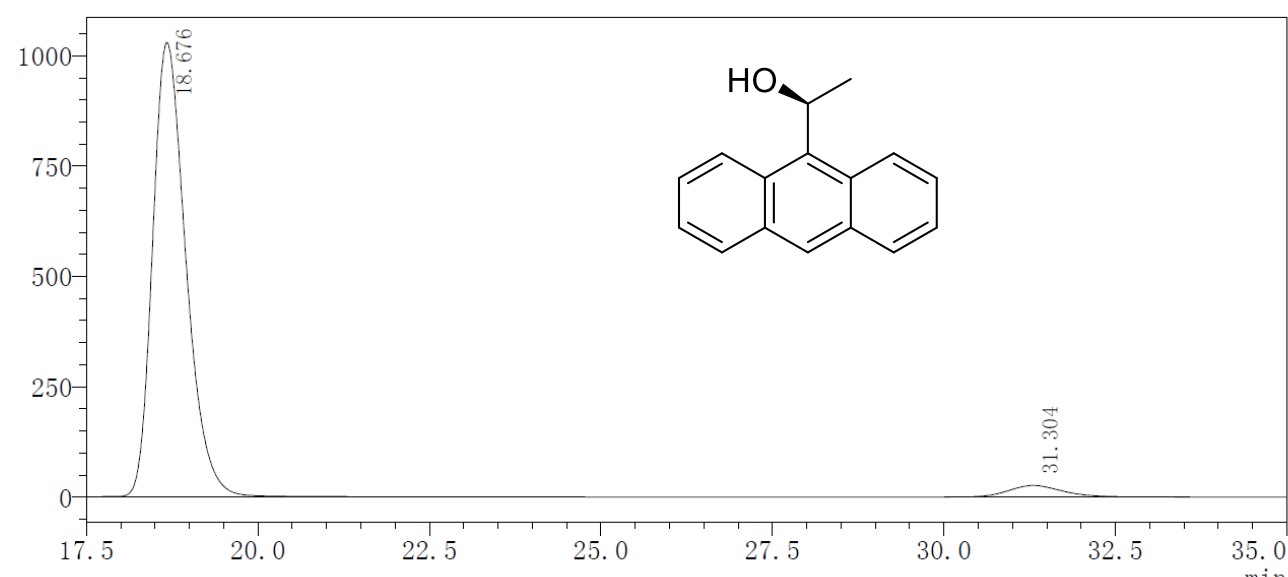

\begin{tabular}{|r|r|r|r|r|r|}
\hline Peak & Ret. Time & \multicolumn{1}{c|}{ Area } & \multicolumn{1}{|c|}{ Area \% } & \multicolumn{1}{c|}{ Height } & \multicolumn{1}{c|}{ Height \% } \\
\hline 1 & 18.676 & 34701746 & 96.062 & 1029406 & 97.544 \\
\hline 2 & 31.304 & 1422724 & 3.938 & 25922 & 2.456 \\
\hline
\end{tabular}




\section{Computational details}

The DFT calculations were performed with the Gaussian $16^{17}$ software package. The $\omega$ B97X-D ${ }^{18}$ was employed to describe all the minima and transition states. Two basis sets were employed, the SDD ${ }^{19}$ for $\mathrm{Mn}$ atom and the cc-pVDZ ${ }^{20}$ basis set for $\mathrm{C}, \mathrm{H}, \mathrm{N}$ and $\mathrm{O}$ were used for the optimization, the other def2-QZVPP ${ }^{21}$ for all atoms was used for more accurate single point calculations. All the calculations were carried out with solvation using the self-consistent reaction field (SCRF) calculations in the conductor-like polarizable continuum model (CPCM) ${ }^{22}$ (2-propanol, $\varepsilon=19.264$ ). Vibrational frequency analysis was performed to confirm the nature of the stationary points (only real frequencies for minima, one imaginary frequency for transition states), and IRC calculations were carried out on the transition states to further confirm their correct identification. Computed harmonic frequencies were used to calculate the thermal contribution to Gibbs free energy at $353.15 \mathrm{~K}\left(80{ }^{\circ} \mathrm{C}\right)$. Multiwfn ${ }^{23}$ software package was used to analysis the non-bond interaction. Geometry structures were drawn in CYLview software. ${ }^{24}$
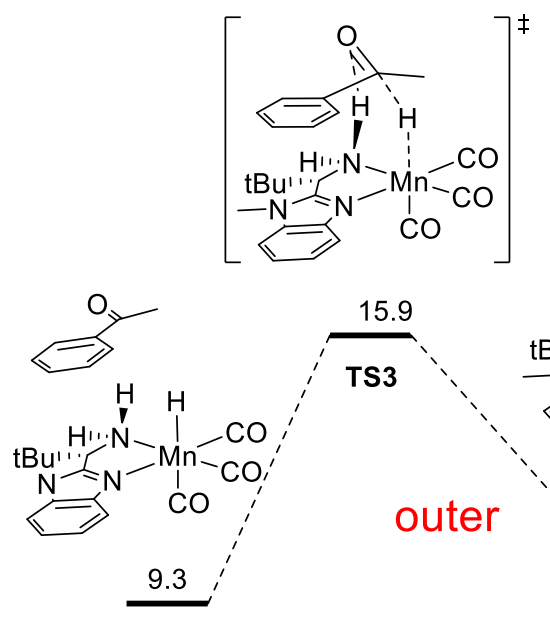

Mn1d

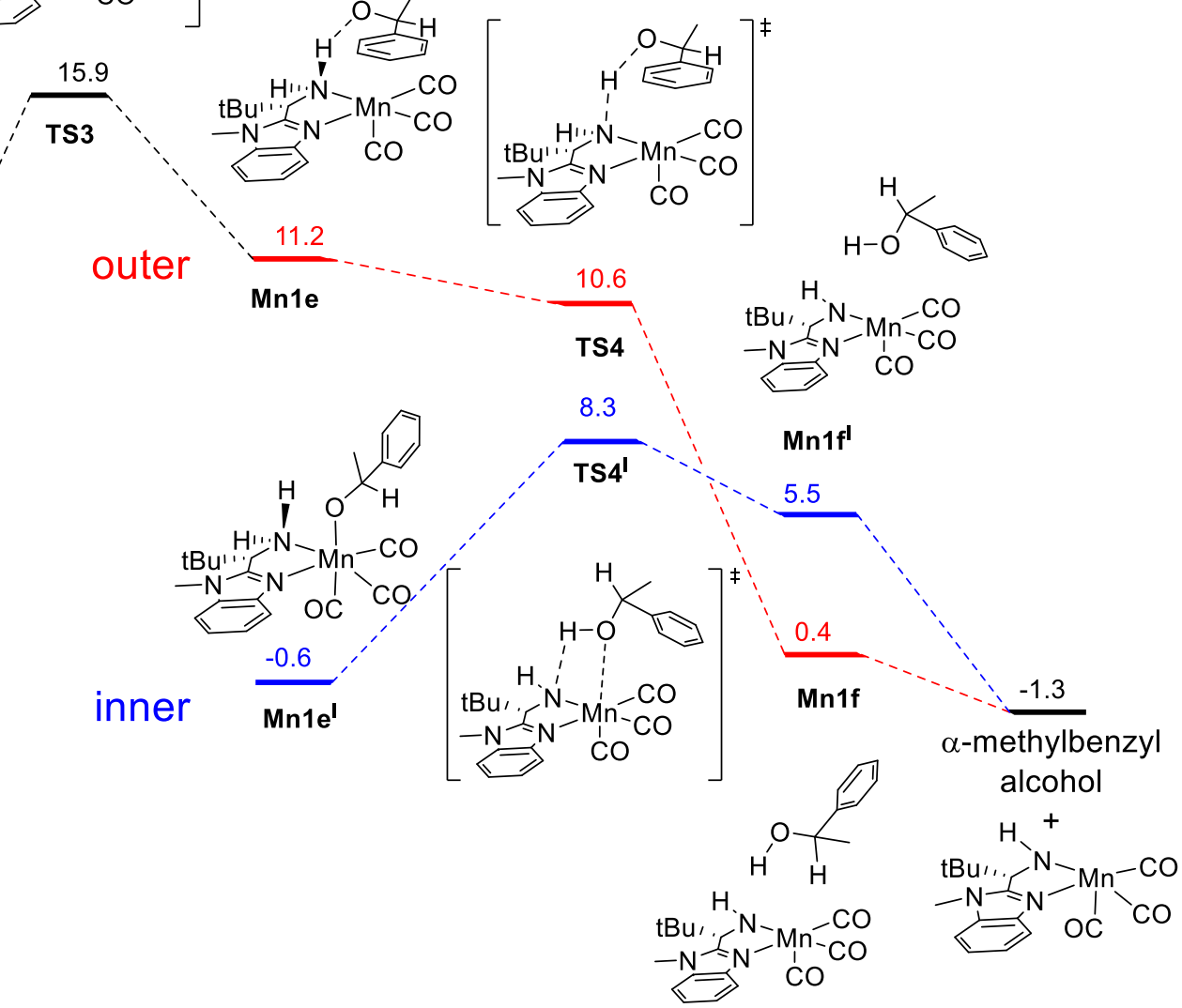

Figure S1. Gibbs free energy profiles for the ATH of acetophenone from $\mathrm{MnH}$. 
Table S2. Energies of intermediates in the proposed catalytic cycle for asymmetric transfer hydrogenation with Mn-1. Absolute energies are given in unit of Hartree, and the relative Gibbs free energies are in $\mathrm{kcal} \mathrm{mol}^{-1}$.

\begin{tabular}{|c|c|c|c|c|c|}
\hline & Freq & ZPE & Cor to G & SPE & $\Delta \mathrm{G}$ \\
\hline iPrOH & 236.02 & 0.108287 & 0.074599 & -194.397713 & \multirow{2}{*}{$0.0^{\text {a }}$} \\
\hline Mn1 & 34.09 & 0.322895 & 0.256482 & -2161.735779 & \\
\hline Mn1a & 20.36 & 0.432449 & 0.356062 & -2356.138899 & 12.3 \\
\hline Mn1a ${ }^{\mathrm{O}}$ & 34.31 & 0.433468 & 0.355923 & -2356.148778 & 6.0 \\
\hline TS1 & -434.03 & 0.429478 & 0.35471 & -2356.136885 & 12.7 \\
\hline Mn1b & 30.05 & 0.433967 & 0.358416 & -2356.155662 & 3.2 \\
\hline TS2 & -635.79 & 0.429339 & 0.354718 & -2356.125776 & 19.7 \\
\hline Mn1c & 20.68 & 0.429735 & 0.350718 & -2356.13577 & 10.9 \\
\hline Mn1d & 20.27 & 0.48433 & 0.400998 & -2547.884239 & 9.3 \\
\hline TS3 $(S)$ & -398.84 & 0.483821 & 0.404792 & -2547.877487 & 15.9 \\
\hline TS3 $(R)$ & -419.79 & 0.48376 & 0.402596 & -2547.871636 & 18.2 \\
\hline Mn1e & 28.04 & 0.484923 & 0.406049 & -2547.886273 & 11.2 \\
\hline Mn1e ${ }^{\mathrm{I}}$ & 18.31 & 0.488274 & 0.407815 & -2547.906758 & -0.6 \\
\hline TS4 & -439.94 & 0.482798 & 0.404366 & -2547.885575 & 10.6 \\
\hline TS4 & -104.43 & 0.486842 & 0.406869 & -2547.891717 & 8.3 \\
\hline Mn1f ${ }^{\mathrm{I}}$ & 15.05 & 0.486972 & 0.401391 & -2547.890712 & 5.5 \\
\hline Mn1f & 12.04 & 0.487142 & 0.403232 & -2547.900694 & 0.4 \\
\hline Phenethanol & 47.94 & 0.162247 & 0.120995 & -386.141819 & $-1.3{ }^{\mathrm{b}}$ \\
\hline
\end{tabular}

${ }^{\mathrm{a}}$ energy of $i$-PrOH and amido Mn1, ${ }^{\mathrm{b}}$ energy of $\alpha$-methylbenzyl alcohol and amido Mn1. 
Table S3. Second order perturbation theory analysis.

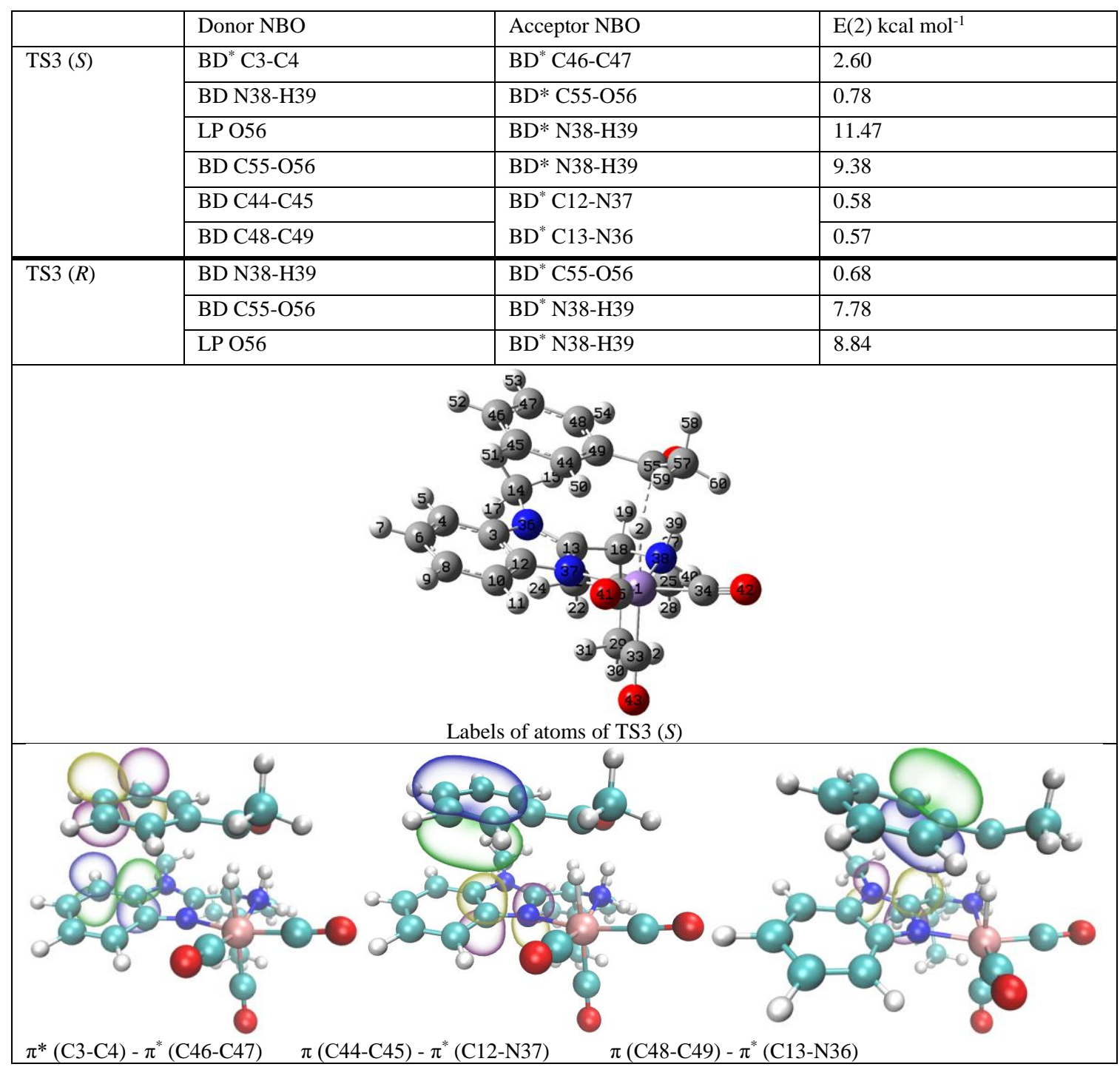




\section{References}

(1) (a) Li, Y.; Ding, K.; Sandoval, C. A. Hybrid $\mathrm{NH}_{2}$-Benzimidazole Ligands for fficient Ru-Catalyzed AsymmetricHydrogenation of Aryl Ketones. Org. Lett. 2009,11, 907-910; (b) Xu, D.; Sun, Q.; Lin, J.; Sun, W. Ligand regulating for manganese-catalyzed enantioselective epoxidation of olefins without acid. Chem. Commun. 2020, 56, 13101-13104; (c) Balboni, G.; Guerrini, R.; Salvadori, S.; Bianchi, C.; Rizzi, D.; Bryant, S. D.; Lazarus, L. H. Evaluation of the Dmt-Tic Pharmacophore: Conversion of a Potent $\delta$-Opioid Receptor Antagonist into a Potent ä Agonist and Ligands with Mixed Properties. J. Med. Chem. 2002, 45, 713-720.

(2) (a) Bischoff, F.; Berthelot, D.; Cleyn, M. D.; Macdonald, G.; Minne, G.; Oehlrich, D.; Pieters, S.; Surkyn, M.; Trabanco, A. A.; Tresadern, G.; Brandt, S. V.; Velter, I.; Zaja, M.; Borghys, H.; Masungi, C.; Mercken, M.; Gijsen. H. J. M.; Design and Synthesis of a Novel Series of Bicyclic Heterocycles As Potent $\gamma$-Secretase Modulators. J. Med. Chem. 2012, 55, 9089-9106; (b) Xu, H. S.; Ding, S. Y.; An, W. K.; Wu, H.; Wang, W. Constructing Crystalline Covalent Organic Frameworks from Chiral Building Blocks. J. Am. Chem. Soc. 2016, 138, 11489-11492.

(3) Zhang, Z. M.; Chen, P.; Li, W.; Niu, Y.; Zhao, X. L.; Zhang, J. A New Type of Chiral Sulfinamide Monophosphine Ligands: Stereodivergent Synthesis and Application in Enantioselective Gold(I)-Catalyzed Cycloaddition Reactions. Angew. Chem., Int. Ed. 2014, 53, 4350-4354.

(4) Bruneau-Voisine, A.; Wang, D.; Dorcet, V.; Roisnel, T.; Darcel, C.; Sortais, J.-B. Transfer Hydrogenation of Carbonyl Derivatives Catalyzed by an Inexpensive Phosphine-Free Manganese Precatalyst. Org. Lett. 2017, 19, 3656-3659.

(5) Zhang, L.; Tang, Y.; Han, Z.; Ding, K. Lutidine-Based Chiral Pincer Manganese Catalysts for Enantioselective Hydrogenation of Ketones. Angew. Chem., Int. Ed. 2019, 58, 4973-4977.

(6) Li, Y.; Yu, S.; Wu, X.; Xiao, J.; Shen, W.; Dong, Z.; Gao, J.; Iron Catalyzed Asymmetric Hydrogenation of Ketones. J. Am. Chem. Soc. 2014, 136, 4031-4039.

(7) Chen, X.; Lu, Z. Iminophenyl Oxazolinylphenylamine for Enantioselective Cobalt- Catalyzed Hydrosilylation of Aryl Ketones. Org. Lett. 2016, $18,4658-4661$.

(8) Utsumi, N.; Arai, N.; Kawaguchi, K.; Katayama, T.; Yasuda, T.; Murata, K.; Ohkuma, T. Asymmetric Hydrogenation of Polysubstituted Aromatic Ketones Catalyzed by the DIPSkewphos/PICA Derivative-Ruthenium(II) Complexes. ChemCatChem 2018, 10, 3955 -3959.

(9) Sun, Y.; Lu, C.; Zhao, B.; Xue, M. Enantioselective Hydroboration of Ketones Catalyzed by Rare-Earth Metal Complexes Containing Trost Ligands. J. Org. Chem. 2020, 85, 10504-10513.

(10) Widegren, M. B.; Harkness, G. J.; Slawin, A. M. Z.; Cordes, D. B.; Clarke, M. L. A Highly Active Manganese Catalyst for Enantioselective Ketone and Ester Hydrogenation. Angew. Chem., Int. Ed. 2017, 56, 5825-5828.

(11) Flinois, K.; Yuan, Y.; Bastide, C.; Harrison-Marchand, A.; Maddaluno, J. Enantioselective protonation and alkylation of non-covalent mixed aggregates of chiral 3-aminopyrrolidine lithium amides. Tetrahedron 2002, 58, 4707-4716.

(12) Yeung, C. T.; Yeung, H. L.; Chan, W. T. K.; Yan, S. C.; Tam, E. C. Y.; Wong, K. L.; Lee, C. S.; Wong, W. T. Stereolabile Chiral Biphenyl Hybrids: Crystallization-Induced Dynamic Atropselective Resolution Involving Supramolecular Interactions. CrystEngComm 2013, 15, 836840.

(13) Fujihara, T.; Semba, K.; Terao, J.; Tsuji, Y. Copper-Catalyzed Hydrosilylation with a Bowl-Shaped Phosphane Ligand: Preferential Reduction of a Bulky Ketone in the Presence of an Aldehyde. Angew. Chem., Int. Ed. 2010, 49, 1472-1476.

(14) Qi, S. B.; Li, M.; Li, S.; Zhou, J. N.; Wu, J. W.; Yu, F.; Zhang, X. C.; Chan, A. S.; Wu, J. Copper-dipyridylphosphine-catalyzed hydrosilylation: enantioselective synthesis of aryl- and heteroaryl cycloalkyl alcohols. Org. Biomol. Chem. 2013, 11, 929-937.

(15) Adams, H.; Elsunaki, T. M.; Ojea-Jimenez, I.; Jones, S.; Meijer, A. J. Diastereoselective Cycloadditions and Transformations of N-Alkyl and N-Aryl Maleimides with Chiral 9-Anthrylethanol Derivatives. J. Org. Chem. 2010, 75, 6252-6262.

(16) Putten, R.; Benschop, J.; Munck, V. J.; Weber, M.; Muller, C.; Filonenko, G. A.; Pidko, E. A. Efficient and Practical Transfer Hydrogenation of Ketones Catalyzed by a Simple Bidentate Mn-NHC Complex. ChemCatChem 2019, 11, 5232-5235.

(17) Frisch, M. J.; Trucks, G. W.; Schlegel, H. B.; Scuseria, G. E.; Robb, M. A.; Cheeseman, J. R.; Scalmani, G.; Barone, V.; Petersson, G. A.; Nakatsuji, H.; Li, X.; Caricato, M.; Marenich, A. V.; Bloino, J.; Janesko, B. G.; Gomperts, R.; Mennucci, B.; Hratchian, H. P.; Ortiz, J. V.; Izmaylov, A. F.; Sonnenberg, J. L.; Williams; Ding, F.; Lipparini, F.; Egidi, F.; Goings, J.; Peng, B.; Petrone, A.; Henderson, T.; Ranasinghe, D.; Zakrzewski, V. G.; Gao, J.; Rega, N.; Zheng, G.; Liang, W.; Hada, M.; Ehara, M.; Toyota, K.; Fukuda, R.; Hasegawa, J.; Ishida, M.; Nakajima, T.; Honda, Y.; Kitao, O.; Nakai, H.; Vreven, T.; Throssell, K.; Montgomery Jr, J. A.; Peralta, J. E.; Ogliaro, F.; Bearpark, M. J.; Heyd, J. J.; Brothers, E. N.; Kudin, K. N.; Staroverov, V. N.; Keith, T. A.; Kobayashi, R.; Normand, J.; Raghavachari, K.; Rendell, A. P.; Burant, J. C.; Iyengar, S. S.; Tomasi, J.; Cossi, M.; Millam, J . M.; Klene, M.; Adamo, C.; Cammi, R.; Ochterski, J. W.; Martin, R. L.; Morokuma, K.; Farkas, O.; Foresman, J. B.; Fox, D. J. Wallingford, CT, 2016.

(18) Chai, J. D.; Head-Gordon, M. Long-range corrected hybrid density functionals with damped atom-atom dispersion corrections Phys. Chem. Chem. Phys. 2008, 10, 6615-6620. 
(19) Dolg, M.; Wedig, U.; Stoll, H. Preuss. Energy-adjusted ab initio pseudopotentials for the first row transition elements. J. Chem. Phys. 1987, $86,866-872$.

(20) Dunning, T. H. Gaussian basis sets for use in correlated molecular calculations. I. The atoms boron through neon and hydrogen. J. Chem. Phys. 1989, 90, 1007-1023.

(21) Weigend, F.; Furche, F.; Ahlrichs, R. Gaussian basis sets of quadruple zeta valence quality for atoms H-Kr. J. Chem. Phys. 2003, 119, 12753-12762.

(22) Barone, V.; Cossi, M. Quantum Calculation of Molecular Energies and Energy Gradients in Solution by a Conductor Solvent Model. J. Phys. Chem. A. 1998, 102, 1995-2001.

(23) Lu, T.; Chen, F. Multiwfn: A multifunctional wavefunction analyzer. J. Comput. Chem. 2012, 33, 580-592.

(24) Legault, C. Y. Université de Sherbrooke, CYLview, 1.0b, 2009 (http://www.cylview.org). 


\section{Coordinates}

\section{Mn1a}

Mn -0.14239-0.09613 -0.15305

$\mathrm{H}-0.561950 .82743 .48027$

C $3.89286-0.002961 .30515$

C 5.025360 .618051 .8351

H 5.838590 .046252 .28294

C 5.069592 .005051 .75775

H 5.936632 .534562 .15551

C 4.020812 .741081 .17259

H 4.09599 3.828641 .12731

C 2.89532 .114860 .65133

H 2.086982 .690540 .20175

C 2.837020 .719070 .72561

C $2.31895-1.388650 .61225$

C $4.24797-2.402721 .90934$

H $3.59797-3.276652 .01361$

H $4.53079-2.054792 .91194$

H $5.15153-2.687371 .35425$

C $1.48616-2.592740 .27644$

H $1.59471-3.31351 .11567$

C $1.9846-3.37726-0.99599$

C $3.44648-3.81628-0.84769$

H $3.74114-4.42282-1.718$

H $3.59926-4.436330 .04896$

H $4.13119-2.95574-0.804$

C $1.12268-4.64197-1.13121$

H $0.06287-4.40608-1.31113$

H $1.18636-5.26648-0.22565$

H $1.46909-5.24585-1.98424$

C $1.84988-2.5335-2.26486$

H $2.23651-3.09358-3.13081$

H $2.42053-1.59492-2.18852$

H $0.80029-2.2822-2.4651$

C $0.214480 .1946-1.87341$

C $-1.89245-0.36459-0.52075$

C -0.404941 .6690 .13758$

N $3.52944-1.338151 .23265$

N $1.86109-0.178430 .32512$

N $0.12464-2.082870 .20084$

$\mathrm{H}-0.41745-1.43691 .65994$

H $-0.46081-2.77016-0.26813$

O -0.616432 .780770 .35737$

O $-2.99504-0.57573-0.7691$

O $0.446890 .43188-2.97819$

C $-0.35775-0.247753 .34574$

O $-0.72499-0.534262 .00041$

C - $1.25437-1.041054 .28958$

H $-1.05962-0.768535 .33819$

H $-2.31409-0.846594 .0695$

H - $1.06915-2.122114 .176$

C $1.11971-0.50333 .61513$

H $1.35433-0.305934 .67208$

H $1.37635-1.554923 .40257$

H 1.753580 .147782 .999

\section{TS1}

Mn -0.15949 -0.09282 -0.10568

H - 0.417920 .830473 .32641

C 3.905250 .011241 .30307

C 5.040830 .640761 .81683

H 5.86920 .074512 .24343

C 5.067152 .028541 .75135

H 5.93542 .564852 .13714

C 3.998542 .757451 .19311

H 4.060393 .846151 .15621
C 2.870582 .122970 .68822 H 2.047682 .693190 .25872 C 2.829720 .725730 .75119 C $2.33508-1.385590 .62695$ C $4.29885-2.388581 .88044$ H $3.66204-3.271541 .98838$ H $4.59284-2.045042 .88127$ H $5.19702-2.657051 .30884$ C $1.51444-2.603260 .30619$ H 1.64308 -3.32072 1.14347 C $1.98455-3.37894-0.97853$ C $3.45372-3.80395-0.86547$ H $3.73435-4.40009-1.74731$ H $3.63244-4.42960 .02231$ H $4.13026-2.93672-0.82998$ C $1.131-4.65123-1.09669$ H $0.06616-4.42593-1.25977$ H $1.21588-5.27428-0.19201$ H $1.46839-5.25256-1.9549$ C $1.81265-2.53206-2.241$

H $2.17794-3.08914-3.11788$ H $2.38316-1.59266-2.17725$ H $0.75735-2.2833-2.41338$ C $0.222160 .21349-1.82706$ C $-1.9075-0.35551-0.49433$ C -0.433821 .66370 .20805$ N $3.55686-1.327321 .22381$ N $1.8574-0.178940 .35899$ $\mathrm{N} 0.14326-2.111970 .25518$ H $-0.30675-1.557121 .46487$ H $-0.45838-2.81746-0.16316$ O -0.65372 .767310 .45627$ O $-3.00902-0.55765-0.75303$ O $0.467620 .47126-2.92359$ C $-0.32651-0.268853 .25409$ O $-0.69034-0.623291 .93328$ C - $1.3209-0.900034 .2251$ H - $1.12463-0.579835 .26038$ H - $2.34991-0.614353 .96162$ H - $1.24696-1.999544 .18441$ C $1.10905-0.653573 .60241$ H $1.31312-0.450934 .66505$ H $1.27519-1.729913 .42536$ H $1.83242-0.080533 .00821$

\section{Mn1b}

Mn -0.2304 -0.17174 0.01417 H 0.828071 .0212 .59377 C 3.861950 .011721 .31799 C 4.970870 .664991 .86025 H 5.800820 .11692 .30698 C 4.968552 .052591 .79517 H 5.814352 .607652 .20362 C 3.92 .758381 .20652 H 3.940113 .848091 .16965 C 2.800722 .100070 .67165 H 1.9782 .652060 .21874 C 2.786560 .701950 .73716 C $2.34483-1.412540 .59104$ C $4.33141-2.390161 .83701$ H $3.72828-3.298751 .92616$ H $4.62501-2.068752 .84504$ H $5.23154-2.608741 .24719$ C $1.59216-2.666620 .24284$ H $1.75116-3.403881 .04838$ C $2.05089-3.36084-1.0822$
C $3.55379-3.66326-1.02985$ H $3.84868-4.2037-1.94157$ H $3.81302-4.30123-0.17169$ H $4.15699-2.74417-0.98454$ C $1.30118-4.69711-1.20971$ H $0.21347-4.56799-1.3211$ H $1.4821-5.34173-0.33549$ H $1.64937-5.23394-2.10477$ C $1.76351-2.48966-2.30798$ H $2.15421-2.98358-3.21057$ H $2.24566-1.50429-2.22491$ H $0.68605-2.33509-2.45454$ C $-0.069620 .0817-1.76281$

C $-2.01458-0.42772-0.10262$

C -0.494441 .587330 .27258$

N $3.5483-1.334011 .22174$

N $1.84622-0.220510 .31042$ N $0.17373-2.266290 .20784$ H -0.15701 -2.18602 1.18219 H $-0.38051-2.97021-0.27422$ O -0.701582 .703420 .47043$ O -3.1498 - $0.60424-0.14995$ O $0.011480 .3238-2.88787$ C 0.169350 .230253 .01799 O - $0.37521-0.56312 .01217$ C -0.929930 .9433 .81306$ $\mathrm{H}-0.51581 .586324 .60782$ $\mathrm{H}-1.535331 .572753 .14223$ H -1.600180 .201684 .28103$ C $1.04968-0.618293 .9413$ H $1.5024-0.01784 .74795$ H $0.4511-1.422394 .40306$ H $1.86273-1.088283 .36574$

\section{TS2}

Mn -0.29583 -0.15283 -0.02883 H $-0.44586-0.223761 .60564$ C 3.736650 .024551 .42663 C 4.827140 .67572 .00724

H 5.644560 .125472 .47393 C 4.822872 .063871 .95463 H 5.6552 .617222 .39243 C 3.769352 .772311 .34292 H 3.807083 .862451 .31796 C 2.687652 .116270 .77 H 1.877062 .670360 .2986 C 2.676760 .71770 .81983 C $2.24283-1.396810 .64835$ C $4.18442-2.381181 .95555$ H $3.57503-3.28772 .01887$ H $4.44248-2.065042 .97477$ H $5.10442-2.600031 .39738$ C $1.49527-2.642280 .26853$ H $1.61139-3.376011 .0826$ C $1.9992-3.3347-1.0401$ C $3.49881-3.64059-0.93666$ H $3.82383-4.17799-1.83991$ H $3.72688-4.2826-0.07287$ H $4.10155-2.72275-0.86689$ C $1.25161-4.66901-1.19663$ H $0.16682-4.53879-1.33309$ H $1.40884-5.31843-0.32148$ H $1.62195-5.2005-2.08594$ C $1.76024-2.45977-2.27469$ H $2.19023-2.94946-3.16155$ H $2.23448-1.47265-2.16822$ 
H $0.68994-2.30755-2.46942$ C $-0.141720 .08208-1.81786$ C $-2.06305-0.43165-0.09304$ C -0.563571 .579340 .30754$ N $3.42617-1.320671 .31673$ N $1.75113-0.203920 .35951$ N $0.0756-2.232450 .1908$ H $-0.31673-2.402131 .13384$ H $-0.42102-2.84935-0.44896$ O -0.781382 .674560 .60701$ O $-3.19925-0.63589-0.08495$ O $-0.105370 .33454-2.94397$ C $-0.45558-0.952453 .06877$ O $-0.2761-2.178982 .8815$ C $-1.85284-0.45083 .40396$ H - 1.949920 .634423 .26296 H -2.60032 -0.97107 2.79119 H - $2.04941-0.684524 .46473$ C $0.68921-0.114783 .6177$ H 0.580790 .952823 .38446 H $0.69583-0.233614 .71531$ H $1.64616-0.484553 .22868$

\section{Mn1c}

Mn -0.10193 0.13336 -0.34019 H -0.337780 .303041 .23043$ C 3.90260 .001181 .2726 C 5.030470 .582891 .85497 H $5.77792-0.011472 .38121$ C 5.15781 .960521 .72588 H 6.024092 .459882 .16245 C 4.193872 .725271 .03946 H 4.332633 .804430 .95733 C 3.073482 .138220 .4651 H $2.33032 .73622-0.06051$ C 2.930540 .751340 .58967 C $2.29711-1.315490 .53452$ C $4.0807-2.393591 .98059$ H $3.35397-3.198162 .13069$ H $4.38321-2.014842 .96608$ H $4.96311-2.78771 .45936$ C $1.44573-2.513240 .22512$ H 1.48257 -3.18724 1.09398 C $1.90332-3.35116-1.01258$ C $3.3647-3.78567-0.8414$ H $3.65687-4.42373-1.68868$ H $3.51169-4.371950 .07794$ H $4.04988-2.92481-0.82712$ C $1.03566-4.61866-1.08136$ H - $0.02873-4.39926-1.25894$ H $1.11146-5.20421-0.15195$ H $1.37173-5.25509-1.91356$ C $1.77384-2.56586-2.32078$ H $2.17828-3.16445-3.15126$ H $2.33152-1.61835-2.28001$ H $0.72634-2.33781-2.55991$ C $0.113240 .13209-2.14528$ C $-1.880240 .03084-0.3888$ C $-0.187711 .90324-0.23064$ N $3.46647-1.311371 .23419$ N $1.93258-0.104310 .15359$ N $0.0689-1.984360 .0873$ H - $0.37318-2.076571 .00297$ H - $0.46259-2.58277-0.54219$ O $-0.286953 .04904-0.0857$ O -3.0341 $-0.05243-0.35143$ O $0.160830 .27785-3.29266$ C $0.2623-0.990183 .53725$ O $0.41536-2.084273 .01666$
C - $1.0962-0.500433 .96167$ $\mathrm{H}-1.235130 .550163 .66977$ H - $1.88523-1.126253 .52721$ H - $1.15644-0.54775 .06176$ C $1.42605-0.070793 .7939$ H 1.424680 .688882 .99432 H 1.323160 .454844 .75385 H $2.37208-0.62413 .75588$

\section{Mn1d}

Mn -0.06212 0.13028 -0.30059 $\mathrm{H}-0.267930 .445421 .23321$ C 4.024870 .369451 .09588 C 5.145811 .062761 .55751 H 5.987190 .551732 .0264 C 5.137552 .441841 .39219 H 5.991153 .026651 .73851 C 4.050723 .100690 .78326 H 4.082994 .185540 .67036 C 2.942612 .401840 .32208 H $2.105332 .91897-0.14384$ C 2.935611 .011530 .48564 C $2.47572-1.101330 .55228$ C $4.5283-1.977541 .78032$ H $3.91799-2.840872 .06316$ H $4.95415-1.546562 .69428$ H $5.34282-2.304611 .12055$ C $1.67095-2.365920 .40948$ H $1.80379-2.952551 .33492$ C $2.07365-3.31105-0.77305$ C $3.5647-3.66114-0.71237$ H $3.80456-4.3602-1.52726$ H $3.83348-4.155610 .23215$ H $4.19958-2.77304-0.84646$ C $1.27691-4.61903-0.63324$ H $0.18904-4.47115-0.71457$ H $1.48067-5.106910 .3327$ H $1.56461-5.31644-1.43403$ C $1.78638-2.67117-2.13503$ H $2.14755-3.33526-2.93488$ H $2.29764-1.70261-2.23959$ H $0.71259-2.51197-2.30208$ C $0.09909-0.01342-2.10523$ C - $1.8291-0.08659-0.25099$ C $-0.274221 .88881-0.3449$ N $3.70216-0.974231 .1369$ N 1.982830 .060760 .16157 N $0.26021-1.923470 .30776$ H - $0.1746-1.993531 .23244$ H $-0.25376-2.58311-0.27325$ O - $0.461513 .03282-0.29507$ O $-2.97168-0.23184-0.13301$ O $0.09570 .04961-3.26195$ C 1.101730 .730883 .80222 C 2.436480 .876774 .16803 C $3.19208-0.244144 .5133$ C $2.61889-1.515444 .46535$ C $1.29451-1.665424 .06276$ C $0.52286-0.542393 .7433$ H 0.518641 .61233 .5339 H 2.890551 .86874 .18323 H $4.23396-0.124914 .81728$ H $3.20772-2.392674 .73899$ H $0.83501-2.654064 .01497$ C $-0.91016-0.752353 .36191$ O - $1.26731-1.827132 .89637$ C -1.896970 .353353 .62401$ $\mathrm{H}-1.836410 .680964 .67255$ H - 1.65931 .217732 .98718
H -2.9092 0.003453 .39148

TS3 $(S)$

Mn -0.1409 -0.01038 -0.10746

H - 0.301840 .001441 .5195

C 3.936550 .062961 .24844

C 5.080440 .692721 .74158

H 5.895320 .129832 .1975

C 5.129282 .075281 .62506

H 6.003262 .612321 .99696

C 4.075552 .799421 .03216

H 4.154933 .885040 .95535

C 2.94292 .164340 .54144

H 2.13166 2.730350.08569 C 2.878140 .771580 .65929

C $2.3552-1.328110 .60441$

C $4.30849-2.328161 .87848$

H $3.65347-3.188452 .05008$

H $4.64889-1.954672 .85258$

H $5.17624-2.643181 .28388$

C $1.54585-2.557650 .30383$

H $1.63738-3.251681 .15619$

C $1.99532-3.34731-0.97111$

C $3.4788-3.72465-0.87842$

H $3.75384-4.33141-1.75394$

H $3.69414-4.32510 .01769$

H $4.12873-2.83724-0.87834$

C $1.17966-4.64875-1.04303$

H $0.10047-4.47303-1.17241$

H $1.31859-5.25582-0.13502$

H $1.5093-5.2464-1.90589$

C $1.77888-2.52837-2.24793$

H $2.16765-3.08657-3.11304$

H $2.30529-1.56324-2.20182$

H $0.71474-2.33053-2.4359$

C $0.033420 .14543-1.90405$

C - $1.91782-0.21543-0.16456$

C -0.335581 .740580 .16335$

N $3.57084-1.271271 .21311$

N $1.89842-0.13060 .28096$

N $0.14481-2.090280 .20815$

H $-0.27503-2.213871 .1457$

H $-0.37302-2.70441-0.41714$

O -0.506782 .853470 .42927$

O - $3.06247-0.36736-0.14692$

O $0.088830 .35497-3.03836$

C 1.14381 .20363 .55208

C 2.336861 .66234 .10183

C 3.242310 .760494 .66268

C $2.95164-0.602784 .65855$

C $1.76082-1.061344 .09579$

C $0.84028-0.162733 .55028$

H 0.457061 .915573 .09102

H 2.568742 .728314 .08084

H 4.178051 .121215 .09349

H $3.65392-1.31345 .09971$

H $1.51861-2.125054 .08457$

C $-0.47209-0.716333 .03347$

O $-0.57234-1.950412 .85796$

C -1.72690 .086033 .33922$

H - $1.96927-0.07064 .40411$

H - 1.607561 .162673 .1672

H -2.5587 -0.29018 2.73085

TS3 $(R)$

Mn $0.016320 .04876-0.06633$

H - 0.076030 .067221 .57047

C $4.09413-0.132211 .25054$

C 5.263690 .426411 .77037 
H $6.03625-0.185782 .23621$ C 5.397721 .804581 .65894 H 6.295562 .286822 .04829 C 4.401042 .592261 .04888 H 4.547513 .671050 .97659 C 3.240082 .028080 .53637 H 2.473852 .643110 .06639 C 3.089840 .640980 .64609 C $2.4377-1.422750 .58187$ C $4.31589-2.548821 .86687$ H $3.62232-3.386991 .98457$ H $4.63908-2.223242 .86438$ H $5.19003-2.878781 .29005$ C $1.55383-2.599180 .28356$ H $1.62805-3.308451 .12365$ C $1.9325-3.39041-1.01115$ C $3.39775-3.8404-0.94883$ H $3.63232-4.44029-1.84067$ H $3.59548-4.47003-0.06865$ H $4.09038-2.98554-0.93717$ C $1.0512-4.64807-1.08078$ H -0.01982 -4.41484 -1.18442 H $1.17807-5.27329-0.18336$ H $1.33136-5.25028-1.95786$ C $1.73087-2.54635-2.27361$ H $2.07534-3.1117-3.15277$ H $2.30296-1.60762-2.22769$ H $0.67347-2.29717-2.43658$ C $0.1160 .19649-1.86823$ C $-1.7736-0.04415-0.0468$ C -0.060781 .809470 .21082$ N $3.64526-1.441731 .20944$ N $2.06002-0.198540 .25486$ N $0.18042-2.048820 .23919$ H -0.18949 -2.13853 1.20019 H $-0.40269-2.63658-0.3533$ O -0.154572 .930210 .47949$ O -2.92294 -0.12274 0.01125 O $0.130410 .4011-3.00465$ C - 1.616991 .407593 .54756 C -2.878991 .922423 .83474$ C -3.972181 .064983 .97656$ C -3.79338 -0.3102 3.82866 C $-2.52985-0.822893 .5385$ C -1.431780 .028553 .3936$ H -0.777712 .092933 .42136$ H - 3.011273 .000273 .94676 H -4.96059 1.470044 .20178 H -4.64275 -0.98731 3.93969 H -2.37309-1.89563 3.42004 C $-0.08078-0.58853 .08518$ O $-0.00562-1.832112 .95953$ C 1.156710 .172293 .53565 H 1.189621 .211333 .1869 H 1.172650 .171824 .63869 H $2.04788-0.356133 .17599$

\section{Mn1e}

Mn -1.4452 0.34547 -1.1466 H - 1.656480 .364920 .70667 C 2.558360 .45660 .31857 C 3.687061 .089050 .84022 H 4.470180 .53321 .35603 C 3.762392 .465110 .67292 H 4.625813 .00471 .06489 C 2.749553 .179010 .00239 H $2.84994 .2591-0.11516$ C $1.630782 .54057-0.51556$ H $0.854363 .10064-1.03483$
C $1.538481 .15574-0.34306$ C $0.98782-0.94454-0.32913$ C $2.86729-1.913511 .06105$ H $2.19357-2.759751 .2285$ H $3.15938-1.507082 .03771$ H $3.75916-2.255990 .51997$ C $0.16305-2.16365-0.61525$ H $0.21661-2.828720 .26466$ C $0.63989-3.00204-1.84747$ C $2.10875-3.41209-1.68398$ H $2.40436-4.05455-2.5268$ H $2.27322-3.98604-0.75983$ H $2.78029-2.54057-1.68563$ C $-0.20792-4.28265-1.91065$ H - $1.27486-4.07874-2.08997$ H $-0.12353-4.86256-0.9785$ H $0.13818-4.91795-2.73964$ C $0.48939-2.2197-3.1556$ H $0.89584-2.81177-3.98956$ H $1.0356-1.26479-3.12191$ H $-0.56405-2.00853-3.38465$ C - $1.242710 .49591-2.92113$ C $-3.24150 .13835-1.30299$ C $-1.634522 .13588-0.97733$ N $2.17429-0.874550 .32232$ N $0.558360 .24892-0.71837$ $\mathrm{N}-1.21659-1.65997-0.7467$ $\mathrm{H}-1.62593-1.691310 .29276$ H - $1.76739-2.31926-1.29312$ O $-1.801393 .26478-0.83218$ O $-4.37527-0.01476-1.39475$ O $-1.113910 .64289-4.05427$ C -0.08781 .617452 .4876$ C 1.06911 .993543 .16485 C 1.816341 .0383 .85616 C $1.39939-0.292383 .85567$ C $0.24161-0.66473 .16911$ C -0.519540 .285812 .48925$ $\mathrm{H}-0.653032 .370721 .93175$ H 1.399923 .033593 .13939 H 2.726671 .329894 .38358 H $1.98031-1.046814 .39184$ H - $0.09792-1.701223 .14326$ C - $1.79823-0.155551 .76581$ O $-1.95467-1.473351 .65967$ C -3.03724 0.579542.3038 H -3.23113 0.25214 3.33759 H -2.91677 1.6742 .30039 H -3.91099 0.320831 .68725

\section{TS4}

Mn -1.43507 0.34138 -1.17619 H - 1.674970 .396570 .71961 C 2.559060 .465310 .312 C 3.685721 .09910 .83625 H 4.468420 .544131 .35365 C 3.759712 .475450 .66969 H 4.621583 .015951 .06382 C $2.747483 .1882-0.00271$ H $2.846544 .26849-0.11959$ C $1.630652 .54833-0.52346$ H $0.854533 .1076-1.04409$ C $1.539821 .16339-0.35179$ C $0.99074-0.93838-0.33744$ C $2.86901-1.904021 .05623$ H $2.19433-2.748981 .22635$ H $3.16282-1.495452 .03147$ H $3.75991-2.249040 .51515$ C $0.15879-2.15336-0.61825$
H $0.21518-2.815420 .26544$ C $0.63479-3.00248-1.84467$ C $2.09986-3.42329-1.67465$ H $2.39365-4.07364-2.51217$ H $2.25713-3.99231-0.74611$ H $2.7781-2.55689-1.67963$ C $-0.22283-4.27656-1.90321$ H - $-1.28721-4.06341-2.08647$ H -0.14604 -4.85171 -0.96733 H $0.12022-4.92002-2.72733$ C $0.49373-2.22724-3.15774$ H $0.8987-2.8265-3.98743$ H $1.04616-1.27566-3.12781$ H $-0.55781-2.01001-3.38908$ C $-1.222740 .50191-2.9445$ C $-3.228440 .12268-1.34949$ C $-1.631882 .13214-1.00571$ N $2.17619-0.866380 .3156$ N $0.561420 .25504-0.72844$ $\mathrm{N}-1.21397-1.63921-0.74548$ H - $1.62683-1.649590 .36883$ $\mathrm{H}-1.77878-2.31042-1.26237$ O - $1.803333 .26084-0.86288$ O $-4.3596-0.04113-1.45335$ O $-1.082730 .6455-4.077$ C -0.083081 .61882 .4965$ C 1.073751 .986893 .17808 C 1.817251 .024263 .86351 C $1.39673-0.304893 .85253$ C $0.23948-0.669783 .16099$ C -0.517910 .288112 .48711$ H -0.646882 .37751 .94646$ H 1.406793 .026373 .16158 H 2.727441 .309694 .39463 H $1.97481-1.064824 .38389$ H - $0.09903-1.706373 .12702$ C - $1.79485-0.134361 .75507$ O $-1.94179-1.463641 .61385$ C -3.037440 .554812 .33692$ H -3.2164 0.18237 3.35792 H -2.92972 1.649722 .37884 H -3.91445 0.311691 .71846

\section{Mn1f}

Mn -1.33342 0.31804-1.265 H - 2.477360 .78461 .59652 C 2.621320 .580820 .34158 C 3.752781 .246170 .81338 H 4.553430 .721311 .33505 C 3.807222 .61710 .59228 H 4.672943 .180290 .94413 C $2.770543 .29575-0.07833$ H $2.853734 .37262-0.2331$ C $1.649272 .624-0.54955$ H $0.847643 .15024-1.06818$ C $1.583861 .24558-0.32903$ C $1.03609-0.85222-0.19523$ C $2.95177-1.75071 .19612$ H $2.27832-2.580651 .43241$ H $3.28276-1.294762 .13726$ H $3.82288-2.129830 .64575$ C $0.16552-2.04688-0.43196$ H $0.15596-2.668720 .48539$ C 0.6677 -2.97297 -1.60391 C $2.04671-3.56264-1.2869$ H $2.32517-4.28974-2.06498$ H $2.05474-4.09262-0.32176$ H $2.82807-2.78917-1.27442$ C $-0.32681-4.13502-1.74883$ 
H $-1.31765-3.79531-2.08555$ H - $0.45035-4.6765-0.79715$ H $0.04071-4.85148-2.49933$ C $0.75127-2.2001-2.92457$ H $1.11093-2.86711-3.7231$ H $1.45033-1.35182-2.85402$ H - $0.23071-1.80815-3.22257$ C $-0.936520 .97346-2.88069$ C $-3.062410 .03387-1.7014$ C - $1.686391 .97443-0.64811$ N $2.23814-0.749950 .42513$ N $0.605280 .31787-0.64145$ $\mathrm{N}-1.14914-1.47279-0.69503$ $\mathrm{H}-1.96043-1.313610 .99652$ H - $1.81173-2.21889-0.89462$ O $-1.93443 .00905-0.20231$ O - $4.15943-0.17037-1.97694$ O - $0.631661 .33019-3.93303$ C -0.19541 .499582 .67289$ C 1.097531 .72113 .1444 C 1.803960 .686833 .75729 C $1.21482-0.572533 .87904$ C $-0.07358-0.794123 .39425$ C -0.797720 .244852 .80184$ H - 0.742432 .313022 .18958 H 1.562592 .700453 .01542 H 2.817670 .857894 .12505 H $1.76519-1.389794 .35069$ H $-0.53439-1.780443 .47562$ C - 2.237830 .024352 .3654 O - $2.45005-1.270961 .8491$ C -3.194120 .221493 .53637$ H - $2.98547-0.519374 .32424$ H -3.08094 1.227113 .96698 H -4.23451 0.094943 .20187

\section{$\mathrm{Mn} 1 \mathrm{a}^{\circ}$}

Mn $-0.20053-0.24268-0.84764$

$\begin{array}{lllll}\mathrm{H} & 0.42987 & 0.2972 & 2.28616\end{array}$

$\begin{array}{llll}\text { C } & 3.45429 & 0.26659 & 1.3074\end{array}$

$\begin{array}{llll}\text { C } & 4.44321 & 1.00842 & 1.95568\end{array}$

$\begin{array}{llll}\mathrm{H} & 5.16887 & 0.54265 & 2.62286\end{array}$

$\begin{array}{llll}\text { C } & 4.46034 & 2.37557 & 1.70447\end{array}$

H $\quad \begin{array}{llll}\text { H } & 21644 & 2.99633 & 2.18735\end{array}$

$\begin{array}{llll}\text { C } & 3.52629 & 2.97779 & 0.83833\end{array}$

H $3.57806 \quad 4.0541 \quad 0.6676$

C $2.54408 \quad 2.23266 \quad 0.19803$

$\begin{array}{llll}\mathrm{H} & 1.82165 & 2.69777 & -0.4733\end{array}$

$\begin{array}{llll}\text { C } & 2.51659 & 0.85694 & 0.44505\end{array}$

$\begin{array}{llll}\text { C } & 2.08088 & -1.26931 & 0.5242\end{array}$

$\begin{array}{llll}\text { C } & 3.78122 & -2.03974 & 2.23558\end{array}$

$\begin{array}{llll}\text { H } & 3.19192 & -2.96076 & 2.26917\end{array}$

H $3.82218 \quad-1.60957 \quad 3.24522$

$\begin{array}{llll}\mathrm{H} & 4.79923 & -2.27479 & 1.89727\end{array}$

$\begin{array}{llll}\text { C } & 1.3124 & -2.51021 & 0.19228\end{array}$

$\begin{array}{llll}\mathrm{H} & 1.23621 & -3.14603 & 1.09612\end{array}$

$\begin{array}{llll}\text { C } & 2.01261 & -3.38962 & -0.91225\end{array}$

$\begin{array}{llll}\text { C } & 3.3462 & -3.93936 & -0.3923\end{array}$

H $3.78419-4.61991 \quad-1.13842$

H $3.21682-4.51126 \quad 0.54033$

$\begin{array}{llll}\mathrm{H} & 4.07926 & -3.13793 & -0.21706\end{array}$

C $1.08811-4.57386-1.22882$

H $\quad 0.15352-4.24671 \quad-1.70807$

H $\quad 0.83284-5.13752-0.31682$

H $\quad \begin{array}{llll}1.58812 & -5.26599 & -1.92359\end{array}$

C $2.26949-2.58325-2.18945$

H $2.7164 \quad-3.23469-2.95609$

H $2.96538-1.7486 \quad-2.01059$

$\begin{array}{llll}\mathrm{H} & 1.33622 & -2.16814 & -2.595\end{array}$

$\begin{array}{llll}\text { C } & 0.30364 & 0.53612 & -2.38178\end{array}$ $\begin{array}{llll}\text { C } & -1.85157 & -0.61559 & -1.47188\end{array}$

$\begin{array}{llll}\text { C } & -0.74137 & 1.32845 & -0.15091\end{array}$

$\begin{array}{llll}\text { N } & 3.14747 & -1.08699 & 1.34243\end{array}$

$\begin{array}{llll}\mathrm{N} & 1.6661 & -0.13354 & -0.01523\end{array}$

$\begin{array}{lllll}\mathrm{N} & 0.00658 & -2.00564 & -0.21289\end{array}$

H $-0.84196-1.32416 \quad 1.47259$

$\begin{array}{llll}\mathrm{H} & -0.62729 & -2.78362 & -0.37834\end{array}$

$\begin{array}{llll}\text { O } & -1.11301 & 2.2889 & 0.36571\end{array}$

$\begin{array}{llll}\text { O } & -2.89958 & -0.87294 & -1.86797\end{array}$

$\begin{array}{lllll}\text { O } & 0.68176 & 0.96943 & -3.38054\end{array}$

$\begin{array}{llll}\text { C } & -0.24878 & -0.23019 & 2.98394\end{array}$

$\begin{array}{llll}\text { O } & -1.26366 & -0.89717 & 2.24607\end{array}$

$\begin{array}{llll}\text { C } & -0.92477 & 0.80973 & 3.86253\end{array}$

$\begin{array}{llll}\mathrm{H} & -0.18281 & 1.34918 & 4.47015\end{array}$

$\begin{array}{llll}\mathrm{H} & -1.46717 & 1.54062 & 3.24551\end{array}$

$\begin{array}{llll}\mathrm{H} & -1.64341 & 0.3249 & 4.54288\end{array}$

C $0.57316 \quad-1.22338 \quad 3.80011$

H $\quad 1.40196 \quad-0.7198 \quad 4.32251$

H $-0.06099 \quad-1.72415 \quad 4.54917$

H 1.00089 -2.00134 3.14931

\section{$\mathrm{Mn} 1 \mathrm{e}^{\mathrm{I}}$}

Mn -1.39913 $0.44494-1.09881$

H $-2.87385 \quad 1.62796 \quad 1.4079$

$\begin{array}{llll}\text { C } & 2.60143 & 0.2033 & 0.45084\end{array}$

$\begin{array}{llll}\text { C } & 3.75017 & 0.73487 & 1.03904\end{array}$

$\begin{array}{llll}\text { H } & 4.48741 & 0.10408 & 1.53673\end{array}$

$\begin{array}{llll}\text { C } & 3.90964 & 2.11227 & 0.95647\end{array}$

$\begin{array}{llll}\mathrm{H} & 4.79186 & 2.57561 & 1.40088\end{array}$

$\begin{array}{llll}\text { C } & 2.95656 & 2.92333 & 0.30993\end{array}$

$\begin{array}{llll}\mathrm{H} & 3.11817 & 4.00161 & 0.26861\end{array}$

$\begin{array}{llll}\text { C } & 1.81809 & 2.38438 & -0.27351\end{array}$

$\begin{array}{lllll}\mathrm{H} & 1.0846 & 3.01941 & -0.76833\end{array}$

$\begin{array}{llll}\text { C } & 1.64403 & 0.999 & -0.19705\end{array}$

$\begin{array}{llll}\text { C } & 0.97459 & -1.05416 & -0.34227\end{array}$

$\begin{array}{llll}\text { C } & 2.75221 & -2.22099 & 1.04821\end{array}$

H $2.05423 \quad-3.06283 \quad 1.08655$

H $2.98902 \quad-1.918892 .07713$

H $3.6734-2.5377 \quad 0.54104$

$\begin{array}{llll}\text { C } & 0.12623 & -2.22475 & -0.75627\end{array}$

H $\quad 0.12939-2.96916 \quad 0.05765$

$\begin{array}{llll}\text { C } & 0.62821 & -2.9682 & -2.04016\end{array}$

$\begin{array}{llll}\text { C } & 2.08502 & -3.41648 & -1.86788\end{array}$

H $2.39741-3.98426-2.75693$

H $\quad 2.21051 \quad-4.07684-0.99691$

H $2.77014 \quad-2.56168 \quad-1.7677$

C $-0.23384-4.22636-2.23624$

H $-1.29215 \quad-3.99474-2.43207$

H $-0.18587-4.88436-1.35464$

H $0.13143-4.79476-3.1046$

$\begin{array}{lllll}\text { C } & 0.53091 & -2.07683 & -3.28221\end{array}$

H $0.9464-2.60978-4.15076$

H $1.09784-1.1428 \quad-3.15413$

H $-0.50962-1.81803-3.52101$

$\begin{array}{llll}\text { C } & -1.09902 & 0.67614 & -2.85966\end{array}$

$\begin{array}{llll}C & -3.18896 & 0.38593 & -1.31091\end{array}$

$\begin{array}{llll}\text { C } & -1.46998 & 2.22856 & -0.8785\end{array}$

$\begin{array}{llll}\mathrm{N} & 2.13836 & -1.09914 & 0.36248\end{array}$

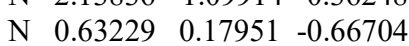

$\mathrm{N}-1.23478-1.68159 \quad-0.9293$

$\begin{array}{llll}\mathrm{H} & -1.68946 & -1.63091 & -0.00747\end{array}$

H $-1.78646-2.29548-1.52421$

$\begin{array}{llll}\text { O } & -1.55717 & 3.37025 & -0.74931\end{array}$

$\begin{array}{llll}O & -4.33442 & 0.32994 & -1.40016\end{array}$

$\begin{array}{lllll}\text { O } & -0.93121 & 0.89436 & -3.98004\end{array}$

$\begin{array}{llll}\text { C } & -1.13773 & 3.08259 & 2.6845\end{array}$

$\begin{array}{llll}\text { C } & -0.09867 & 3.81033 & 3.26987\end{array}$

$\begin{array}{llll}\text { C } & 1.09374 & 3.173 & 3.60988\end{array}$

$\begin{array}{llll}\text { C } & 1.24158 & 1.80856 & 3.35059\end{array}$

$\begin{array}{llll}\text { C } & 0.20463 & 1.09184 & 2.7597\end{array}$

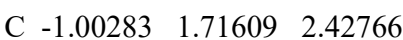
$\begin{array}{llll}\mathrm{H} & -2.06976 & 3.58828 & 2.41607\end{array}$ H $-0.22082 \quad 4.87978 \quad 3.45668$ H $\quad \begin{array}{llll}1.91071 & 3.7383 & 4.06329\end{array}$ $\begin{array}{llll}\mathrm{H} & 2.17991 & 1.30614 & 3.59436\end{array}$ $\begin{array}{llll}\text { H } & 0.32529 & 0.03066 & 2.53037\end{array}$ $\begin{array}{llll}\text { C } & -2.14413 & 0.89622 & 1.82687\end{array}$ $\begin{array}{llll}\text { O } & -1.70362 & 0.00336 & 0.86702\end{array}$ $\begin{array}{llll}\text { C } & -2.87342 & 0.14014 & 2.94618\end{array}$ H $-2.19109 \quad-0.58987 \quad 3.41267$ H $-3.23816 \quad 0.82267 \quad 3.73092$ H $-3.73293 \quad-0.40751 \quad 2.52859$

\section{$\mathrm{TS} 4^{\mathrm{I}}$}

Mn -0.51738 $-0.80943-1.1364$ H $-1.19383 \quad 0.804391 .47869$

$\begin{array}{llll}\text { C } & 2.1694 & 1.1295 & 1.59794\end{array}$

$\begin{array}{llll}\text { C } & 2.68532 & 2.2217 & 2.29756\end{array}$

$\begin{array}{llll}\text { H } & 3.36549 & 2.09594 & 3.1403\end{array}$

$\begin{array}{llll}\text { C } & 2.29039 & 3.48234 & 1.86643\end{array}$

$\begin{array}{llll}\mathrm{H} & 2.66837 & 4.36587 & 2.38291\end{array}$

$\begin{array}{llll}\text { C } & 1.41605 & 3.64379 & 0.7737\end{array}$

$\begin{array}{llll}\mathrm{H} & 1.13405 & 4.65099 & 0.46315\end{array}$

$\begin{array}{llll}\text { C } & 0.90748 & 2.55137 & 0.0823\end{array}$

$\begin{array}{lllll}\mathrm{H} & 0.23071 & 2.68291 & -0.76133\end{array}$

$\begin{array}{llll}\text { C } & 1.2919 & 1.27674 & 0.51195\end{array}$

$\begin{array}{llll}\text { C } & 1.59124 & -0.85361 & 0.82603\end{array}$

$\begin{array}{llll}\text { C } & 3.03822 & -0.82193 & 2.90274\end{array}$

H $2.65505 \quad-1.83076 \quad 3.08743$

$\begin{array}{llll}\text { H } & 2.83678 & -0.21363 & 3.7925\end{array}$

$\begin{array}{llll}\text { H } & 4.11988 & -0.86301 & 2.72043\end{array}$

$\begin{array}{llll}\text { C } & 1.37477 & -2.3201 & 0.59869\end{array}$

H $1.30566 \quad-2.80109 \quad 1.59722$

$\begin{array}{llll}\text { C } & 2.55523 & -3.04971 & -0.1439\end{array}$

$\begin{array}{llll}\text { C } & 3.87343 & -2.89742 & 0.62372\end{array}$

H $4.66026-3.48843 \quad 0.1301$

H $3.78981 \quad-3.26214 \quad 1.65892$

H $4.21631-1.85193 \quad 0.64252$

$\begin{array}{llll}\text { C } & 2.21159 & -4.54541 & -0.20912\end{array}$

H $\quad 1.3094 \quad-4.73704 \quad-0.80952$

$\begin{array}{llll}\mathrm{H} & 2.0504 & -4.96204 & 0.79817\end{array}$

$\begin{array}{llll}\text { H } & 3.03744 & -5.1027 & -0.67775\end{array}$

$\begin{array}{llll}\text { C } & 2.7457 & -2.50932 & -1.56277\end{array}$

H $3.6053-3.00526-2.04008$

H $2.94081 \quad-1.42542-1.56016$

H $1.85883 \quad-2.69698 \quad-2.1818$

$\begin{array}{llll}\text { C } & 0.39951 & -0.55289 & -2.63636\end{array}$

C $-1.74477-1.82419-1.99181$

C $-1.480290 .69714-1.41432$

$\begin{array}{llll}\mathrm{N} & 2.33618 & -0.23451 & 1.77656\end{array}$

$\begin{array}{llll}\mathrm{N} & 0.93822 & 0.01449 & 0.06212\end{array}$

N $0.09503 \quad-2.3718 \quad-0.09256$

H $-0.09803 \quad-3.32936-0.37526$

$\begin{array}{llll}\mathrm{O} & -2.14706 & 1.62561 & -1.55905\end{array}$

$\begin{array}{llll}O & -2.50144 & -2.50023 & -2.53114\end{array}$

$\begin{array}{llll}\text { O } & 1.00406 & -0.37176 & -3.6016\end{array}$

$\begin{array}{llll}\text { C } & -1.73892 & -0.03654 & 1.94534\end{array}$

$\begin{array}{llll}\text { C } & -3.10674 & 0.47528 & 2.38392\end{array}$

$\begin{array}{llll}\mathrm{H} & -3.00216 & 1.30637 & 3.09654\end{array}$

H $-3.66922 \quad 0.834 \quad 1.50916$

$\begin{array}{llll}\mathrm{H} & -3.67787 & -0.33303 & 2.86608\end{array}$

$\begin{array}{llll}\text { C } & -0.91541 & -0.4965 & 3.13499\end{array}$

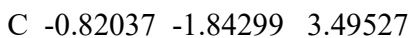

$\begin{array}{llll}\text { C } & -0.26657 & 0.46013 & 3.92502\end{array}$

$\begin{array}{llll}\text { C } & -0.09718 & -2.2258 & 4.62699\end{array}$

H $-1.31678 \quad-2.60139 \quad 2.88716$

$\begin{array}{llll}\text { C } & 0.44182 & 0.08256 & 5.06328\end{array}$

$\begin{array}{llll}\mathrm{H} & -0.31509 & 1.51483 & 3.6408\end{array}$

$\begin{array}{llll}\text { C } & 0.52831 & -1.26496 & 5.41947\end{array}$

H $-0.02967 \quad-3.28263 \quad 4.8929$ 
H $\quad 0.93935 \quad 0.84255 \quad 5.66933$

H $\quad 1.09038 \quad-1.56386 \quad 6.30611$

$\begin{array}{llll}\text { O } & -1.95489 & -1.05448 & 0.98342\end{array}$

H $-1.15851 \quad-1.65421 \quad 0.86732$

\section{Mn1f ${ }^{1}$}

Mn $0.03513-0.17719-0.97117$

H -1.77749 -1.92295 3.46192

$\begin{array}{llll}\text { C } & 3.44716 & 0.03088 & 1.59719\end{array}$

$\begin{array}{llll}\text { C } & 4.40688 & 0.68423 & 2.37212\end{array}$

$\begin{array}{llll}\text { H } & 5.0327 & 0.14889 & 3.08661\end{array}$

$\begin{array}{llll}\text { C } & 4.53087 & 2.05597 & 2.18341\end{array}$

$\begin{array}{llll}\mathrm{H} & 5.2694 & 2.60912 & 2.76546\end{array}$

$\begin{array}{llll}\text { C } & 3.7281 & 2.74743 & 1.25481\end{array}$

$\begin{array}{llll}\mathrm{H} & 3.86179 & 3.82359 & 1.13533\end{array}$

$\begin{array}{llll}\text { C } & 2.77322 & 2.09022 & 0.48893\end{array}$

$\begin{array}{llll}\mathrm{H} & 2.15359 & 2.62531 & -0.23111\end{array}$

$\begin{array}{llll}\text { C } & 2.63675 & 0.71123 & 0.67413\end{array}$

$\begin{array}{llll}\text { C } & 2.07524 & -1.38571 & 0.61276\end{array}$

C $3.51642 \quad-2.31783 \quad 2.47209$

H $2.85672 \quad-3.18881 \quad 2.41614$

H $3.47486-1.908713 .49049$

H $4.54498 \quad-2.62877 \quad 2.24556$ $\begin{array}{llll}\text { C } & 1.27613 & -2.5651 & 0.15283\end{array}$

H $\quad 1.06067 \quad-3.21939 \quad 1.02071$

$\begin{array}{llll}\text { C } & 2.04229 & -3.4589 & -0.89514\end{array}$

C $3.27585-4.10296-0.25088$

H $3.75077 \quad-4.79222 \quad-0.96576$

$\begin{array}{llll}\text { H } & 3.01154 & -4.68759 & 0.64467\end{array}$

$\begin{array}{llll}\mathrm{H} & 4.03243 & -3.35379 & 0.02582\end{array}$

$\begin{array}{llll}\text { C } & 1.09239 & -4.57717 & -1.34788\end{array}$

H $\quad 0.23629-4.18354-1.91574$

H $0.70662-5.14786-0.48756$

$\begin{array}{lllll}\mathrm{H} & 1.62593 & -5.27994 & -2.00606\end{array}$

$\begin{array}{llll}\text { C } & 2.48538 & -2.6388 & -2.11104\end{array}$

$\begin{array}{lllll}\mathrm{H} & 2.99298 & -3.2937 & -2.83583\end{array}$

H $3.18962-1.84057 \quad-1.82845$

H $1.62656 \quad-2.17328 \quad-2.61414$

$\begin{array}{lllll}\text { C } & 0.78455 & 0.55365 & -2.42375\end{array}$

C $-1.54629-0.43296-1.8023$

$\begin{array}{llll}\text { C } & -0.47299 & 1.43659 & -0.35553\end{array}$

$\begin{array}{llll}\mathrm{N} & 3.06326 & -1.30195 & 1.53899\end{array}$

$\begin{array}{llll}\mathrm{N} & 1.78174 & -0.20665 & 0.08832\end{array}$

N $0.05669-1.96887 \quad-0.37646$

H $-0.58412 \quad-2.70297 \quad-0.66834$

$\begin{array}{llll}\text { O } & -0.83715 & 2.43445 & 0.0928\end{array}$
O $-2.55136-0.61714 \quad-2.32861$

$\begin{array}{llll}\text { O } & 1.3177 & 0.95678 & -3.36273\end{array}$

C $-1.4791 \quad-0.95277 \quad 3.01799$

$\begin{array}{llll}\text { C } & 0.02914 & -0.79336 & 3.20975\end{array}$

$\begin{array}{lllll}\mathrm{H} & 0.28428 & -0.75734 & 4.27906\end{array}$

$\begin{array}{lllll}\mathrm{H} & 0.55964 & -1.64885 & 2.76647\end{array}$

$\begin{array}{lllll}\mathrm{H} & 0.38438 & 0.13344 & 2.73048\end{array}$

$\begin{array}{llll}\text { C } & -2.23069 & 0.13809 & 3.75741\end{array}$

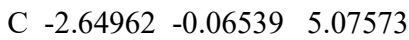

$\begin{array}{llll}\text { C } & -2.4693 & 1.37805 & 3.15687\end{array}$

$\begin{array}{llll}\text { C } & -3.28889 & 0.95069 & 5.78627\end{array}$

H $-2.47541 \quad-1.03354 \quad 5.55317$

$\begin{array}{llll}\text { C } & -3.11033 & 2.39496 & 3.86399\end{array}$

$\begin{array}{llll}\mathrm{H} & -2.14777 & 1.54323 & 2.12835\end{array}$

$\begin{array}{llll}\text { C } & -3.52206 & 2.18588 & 5.18104\end{array}$

$\begin{array}{llll}\mathrm{H} & -3.61201 & 0.77441 & 6.81428\end{array}$

H $-3.29089 \quad 3.35826 \quad 3.38214$

H $-4.02596 \quad 2.98182 \quad 5.73282$

$\begin{array}{llll}\text { O } & -1.8434 & -0.91942 & 1.6527\end{array}$

H $-1.13983-1.3311 \quad 1.10909$ 